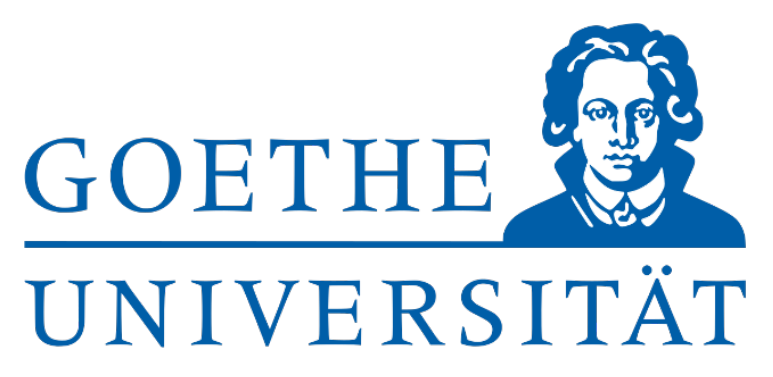

FRANKFURT AM MAIN

\title{
Mitochondrial dysfunction and its consequences in the context of neurodegeneration
}

\author{
Dissertation \\ zur Erlangung des Doktorgrades \\ der Naturwissenschaften \\ vorgelegt beim Fachbereich Biowissenschaften \\ der Johann Wolfgang Goethe-Universität \\ in Frankfurt am Main \\ von \\ Jana Key \\ aus Bergisch Gladbach
}

Frankfurt am Main, 2021

(D30) 
vom Fachbereich Biowissenschaften (15) der Johann Wolfgang Goethe-Universität als Dissertation angenommen.

Dekan: Prof. Dr. Sven Klimpel

Gutachter: Prof. Dr. Enrico Schleiff

Prof. Dr. Georg Auburger

Datum der Disputation: 


\section{ACKNOWLEDGEMENTS}

First of all, I would like to thank my thesis committee members and especially my two thesis reviewers Prof. Georg Auburger and Prof. Enrico Schleiff for taking their time to be present, to read through it all, and help me to improve the quality of my doctoral thesis.

Special thanks go to my mentor Professor Dr. med. Georg Auburger! He has made it possible for me to work on several highly interesting projects, to encounter scientific questions, and pursue my doctoral thesis. I am highly thankful for the close supervision, for the numerous fruitful discussions, and especially for the support throughout tough times with viruses and my pregnancy and early motherhood.

I would also like to thank Dr. rer. nat. Suzana Gispert for sharing all her knowledge in the lab, for teaching, especially the handling of the mice, and for all the helpful discussions we had. I knew there was always an open ear for scientific and personal issues!

My work would not have been as fruitful and rewarding without the perfect colleagues I had. I was honored to supervise two very talented students, Antonia and Aneesha, throughout their theses and it was always a pleasure. Also, the supervision of our interns Robert and Natasha was very rewarding. Also, I was very happy to share the lab with Júlia, Aleksandar, Luis Enrique, Stella, Marina - and as a friend throughout all the time - Nesli. We shared office time, coffee chats, walks, as well as failed and successful experiments and could celebrate together for several occasions.

Great thanks go to Gabi who brought a lot of organization to the lab was always there, and helped whenever there was a problem with an experiment or something that had to be ordered or needed to be taken care of. I am especially thankful for the help with my experiments when I was bound to work from home during Covid19 times and my pregnancy.

My thanks also go to the staff of the animal facility, especially Frau Janton, for taking care of the mice. Special acknowledgment goes to all the mice who made my work possible with their lives. I also want to thank all the supporting people, namely Birgitt, Herr Allerberger, Herr Titok, and the Kögel group for their help and technical input.

I would like to thank all our collaborators who contributed cell lines, performed additional techniques for us, and enriched our hypotheses with their ideas and opinions. Special thanks also go to Professor Helmuth Steinmetz, who made all my work possible by taking me under contract and extending it promptly when necessary.

I am deeply thankful to my parents who supported me throughout my studies and thus made it possible for me to start the Ph.D. at all. They did not understand what I was researching on but shared my ups and downs and proudly reported my publications to their friends. 
The part that my beloved Norman took part in this thesis can hardly be described with words. He was always there to support me, shared frustrations, and manifold paper submissions as well as the great successes. He gave me room for personal time and so often prepared dinner for me after a long day in the lab. Especially the months that we spent working from home side by side were remarkable and full of happiness. I also owe to him that I am mum now of our wonderful boy Cosmo, who was first a passive part of the thesis until he started kicking and is now enriching my life in such an incredible way.
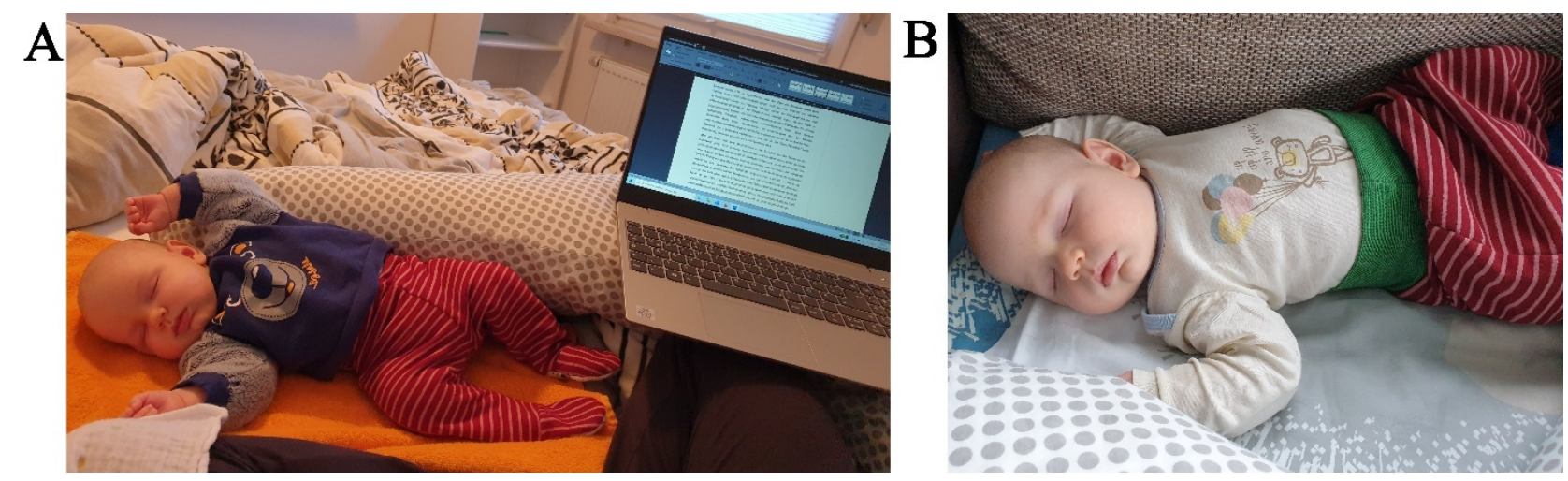

Figure 1: Occasional situations of thesis production. A) Cosmo 8 weeks old while co-working, B) Cosmo 12 weeks old, sleeping alone 
This thesis is based on the following publications:

I: Progression of pathology in PINK1-deficient mouse brain from splicing via ubiquitination, ER stress, and mitophagy changes to neuroinflammation.

Torres-Odio S, Key J, Hoepken HH, Canet-Pons J, Valek L, Roller B, Walter M, Morales-Gordo B, Meierhofer D, Harter PN, Mittelbronn M, Tegeder I, Gispert S, Auburger G. J Neuroinflammation. 2017 Aug 2;14(1):154. doi: 10.1186/ s12974-017-0928-0. PMID: 28768533

II: Systematic Surveys of Iron Homeostasis Mechanisms Reveal Ferritin Superfamily and Nucleotide Surveillance Regulation to be Modified by PINK1 Absence

Key J, Sen NE, Arsović A, Krämer S, Hülse R, Khan NN, Meierhofer D, Gispert S, Koepf G, Auburger G. Cells. 2020 Oct 2;9(10):2229. doi: 10.3390/cells9102229. PMID: 33023155

III: Ubiquitylome profiling of Parkin-null brain reveals dysregulation of calcium homeostasis factors ATP1A2, Hippocalcin and GNA11, reflected by altered firing of noradrenergic neurons

Key J, Mueller AK, Gispert S, Matschke L, Wittig I, Corti O, Münch C, Decher N, Auburger G. Neurobiol Dis. 2019 Jul;127:114-130. doi: 10.1016/j.nbd.2019.02.008. Epub 2019 Feb 11. PMID: 30763678 


\section{TABLE OF CONTENTS}

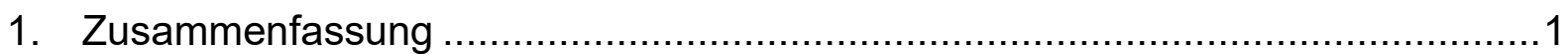

1.1 Mitochondrien und ihre Bedeutung für die Zelle ..................................... 1

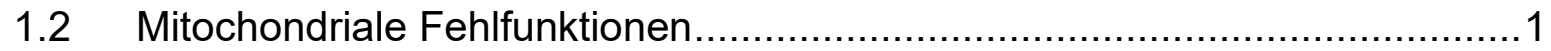

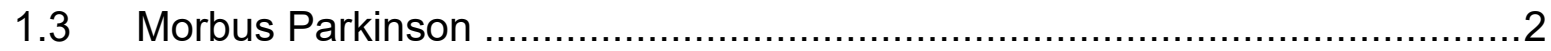

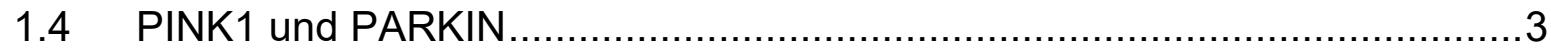

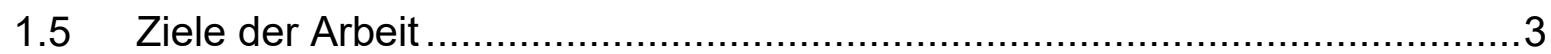

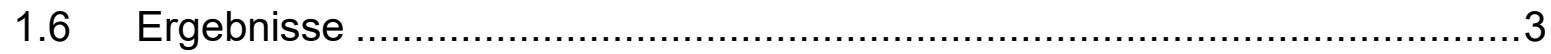

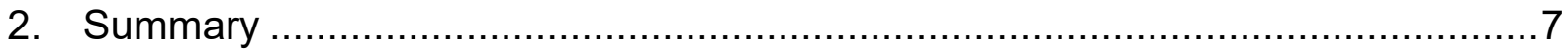

2.1 Mitochondria and their importance for the cell ...................................

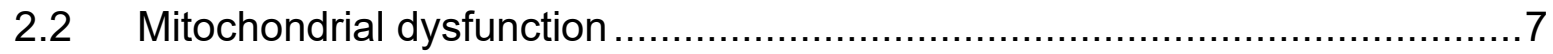

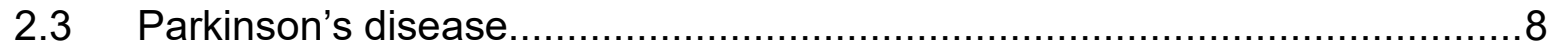

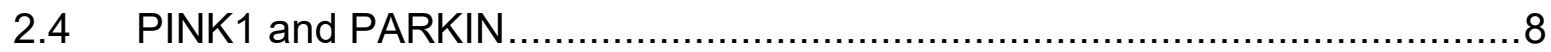

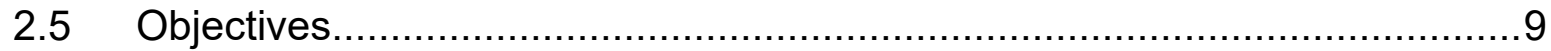

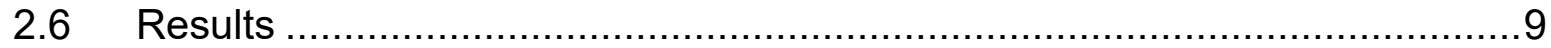

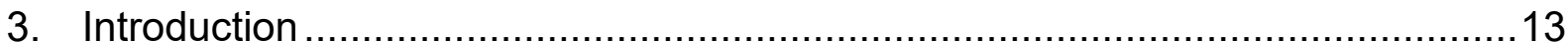

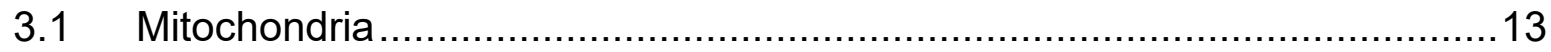

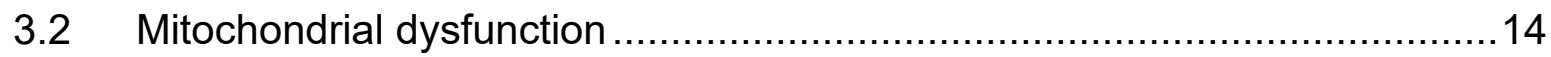

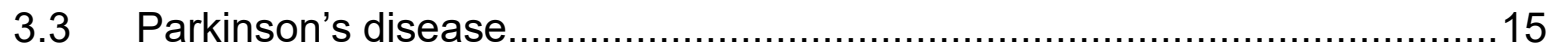

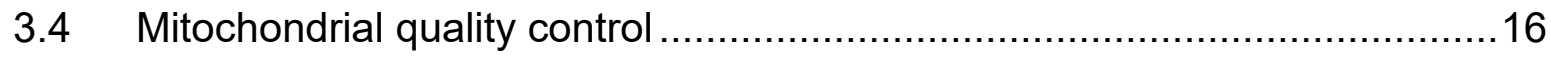

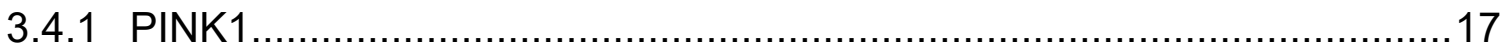

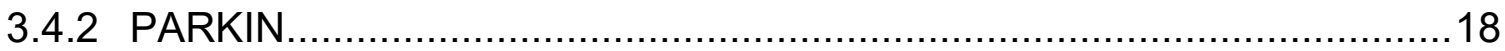

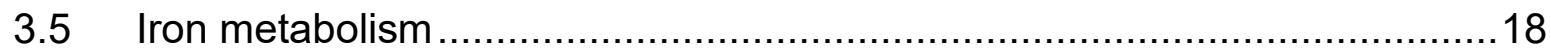

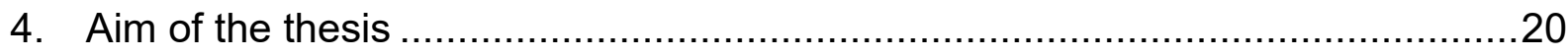

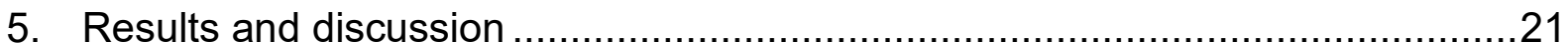

5.1 Progression of pathology in PINK1-deficient tissues .............................21

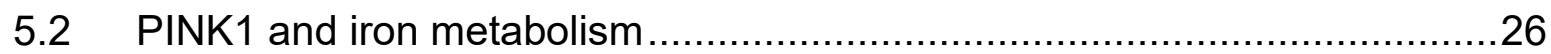

5.3 PARKIN and its substrates - relevance for neurons .................................33

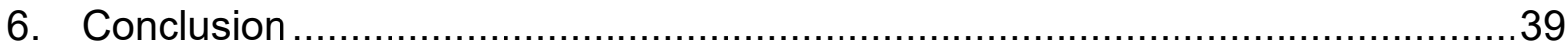

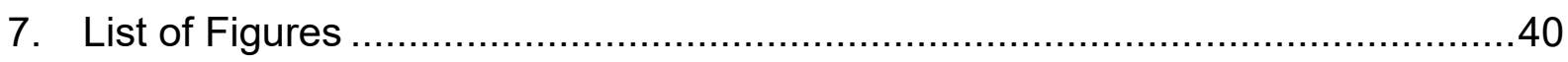

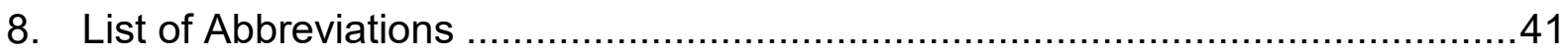

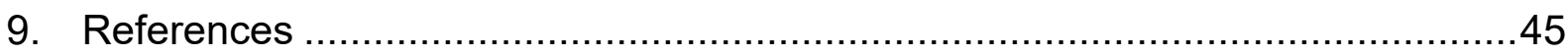

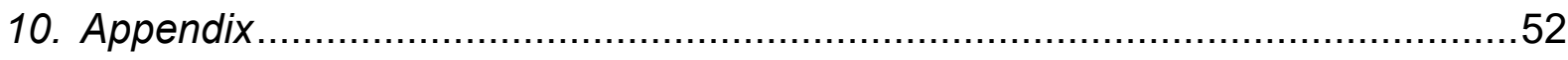

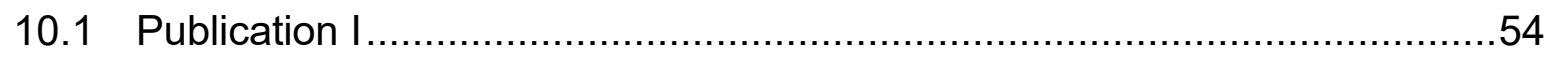

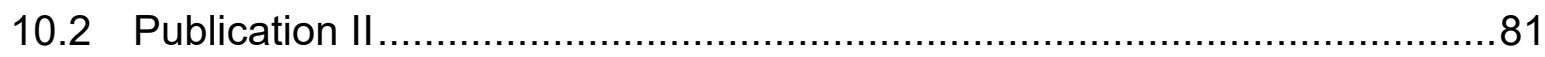




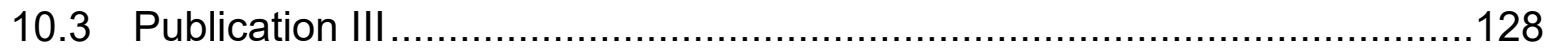

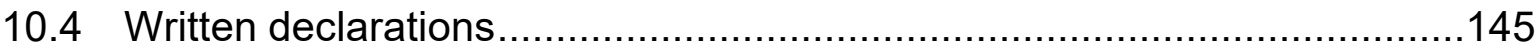

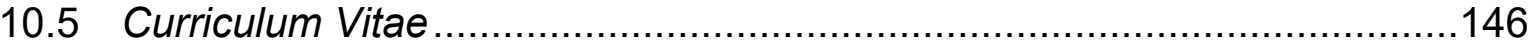




\section{Zusammenfassung}

\subsection{Mitochondrien und ihre Bedeutung für die Zelle}

Seit ihrer Entdeckung im Jahr 1886 stellen Mitochondrien ein Mysterium im Inneren der Zelle dar. Sie wurden später dann als „Kraftwerke der Zellen“ bezeichnet, weil sie mit ihrer Atmungskette und der anschließenden oxidativen Phosphorylierung die Fähigkeit haben, das Energiemolekül ATP zu synthetisieren. Dies dient der Zelle als Energiebaustein. Darüber hinaus spielen Mitochondrien jedoch auch an einer Vielzahl von weiteren Stoffwechselprozessen im Zellalltag eine Rolle. Neben der Energieproduktion sind Mitochondrien erheblich an der Zellgesundheit beteiligt, indem sie eine Rolle im Calciumhaushalt spielen und mit ihren Doppelmembranen Plattformen für diverse zytosolische Vorgänge bieten, insbesondere immunologischer Natur. Eine weitere essenzielle Rolle haben Mitochondrien im Eisenhaushalt inne. Sie können Eisenmoleküle durch eine eigene Ferritin-Form speichern, dienen als ein Ort der Häm-Synthese und sind der Ort der Eisen-Schwefel-Cluster Biogenese, ohne welche die Zelle nicht funktionsfähig wäre.

Man geht davon aus, dass Mitochondrien in der Evolution aus der Symbiose von eukaryoten Zellen mit Prokaryoten hervorgingen und sie daher eine Sonderrolle einnehmen. Sie weisen einen einzigartigen Aufbau auf, da sie, umschlossen von einer Doppelmembran, ihr eigenes Genom beinhalten. Dieses kodiert neben der nukleären DNA für Proteine in den Mitochondrien selbst und ist dementsprechend genauso wichtig für das Überleben der Zellen. Aufgrund ihrer Herkunft im Sinne der Endosymbiontentheorie werden Mitochondrien als Organell wohl toleriert, aber auch sehr penibel kontrolliert, weil ihre Fehlfunktion die Zelle in Alarmbereitschaft versetzen kann, als ob ein Mikroorganismus eingedrungen sei und potenziell bekämpft werden muss. Ein wichtiger Mechanismus dafür ist der selektive Abbau von Mitochondrien, die Mitophagie, durch die nicht richtig funktionierende Mitochondrien markiert und von der Zelle über lysosomale Prozesse abgebaut werden.

\section{$1.2 \quad$ Mitochondriale Fehlfunktionen}

Man sieht die Bedeutsamkeit von Mitochondrien für die Gesundheit von Zellen, Organen und Organismen an der Fülle der Erkrankungen, bei denen mitochondriale 
Störungen eine Rolle spielen und die so gut wie alle Bereiche des Körpers betreffen können. Vorrangig würde man davon ausgehen, dass bei einer Fehlfunktion von Mitochondrien ein respiratorisches Problem und daraus folgend ein Energiemangel herrschen würde. Als Beispiel dafür sind primäre Erkrankungen wie mitochondriale Myopathie, Leigh Syndrom oder MELAS Syndrom zu nennen, denen meist eine Mutation im mitochondrialen oder nukleären Genom zugrundeliegt. Viel bekannter und auch deutlich häufiger sind allerdings Erkrankungen, deren Zusammenhang mit Mitochondrien nicht auf den ersten Blick sichtbar ist und nicht immer liegen Mutationen von Genen für mitochondriale Proteine zugrunde. Dazu zählen z.B. Diabetes Mellitus, psychische Erkrankungen wie Schizophrenie, sowie Krebserkrankungen. Eine große Rolle spielt die mitochondriale Gesundheit aber auch für neurodegenerative Erkrankungen. Insbesondere sind hier Morbus Alzheimer und Morbus Parkinson zu nennen.

In der hier zugrunde liegenden Arbeit sollen die Zusammenhänge zwischen mitochondrialer Gesundheit und der Pathogenese von Parkinson vertieft werden.

\subsection{Morbus Parkinson}

Morbus Parkinson (abgekürzt als PD vom englischen Parkinson's disease) ist als zweithäufigste neurodegenerative Erkrankung von großer Bedeutung in Klinik und Forschung. Die Hauptanzeichen sind Rigidität und Bradykinesie, sowie Tremor und posturale Instabilität. Im Gehirn lässt sich bei Patienten post mortem ein Verlust an dopaminergen Neuronen in der Substantia nigra feststellen, was zu den ersten beiden Anzeichen führt. Zudem gibt es intrazelluläre Einschlüsse in den betroffenen Nervenzellen - Lewy-Körperchen genannt - die aus Alpha-Synuklein und anderen Proteinen wie Ubiquitin zusammengesetzt sind. Außerdem ist der Eisenmetabolismus in Gehirnen von Parkinsonpatienten gestört und es finden sich Eisen-Ablagerungen, vor allem im Mittelhirn.

Die Ursachen für Parkinson sind bislang nicht geklärt. Der Großteil der Fälle ist sporadischer Natur mit unbekannter Ursache und nur bei einem geringen Prozentsatz liegt eine Mutation zugrunde. Die häufigsten Mutationen liegen in den Genen für AlphaSynuklein, PINK1 und PARKIN. 


\section{$1.4 \quad$ PINK1 und PARKIN}

Die Serin-Threonin-Kinase PINK1 und die E3-Ubiquitin-Protein-Ligase PARKIN sind zwei Proteine, die in Stresssituationen an der Mitochondrien-Außenmembran am Abbau von alten oder nicht richtig funktionierenden Mitochondrien beteiligt sind. Im Falle von nicht funktionalen Organellen lagert sich PINK1 an der mitochondrialen Außenmembran an und rekrutiert PARKIN, was dann dazu führt, dass die betroffenen Mitochondrien abgebaut werden. Da PARKIN eine E3-Ubiquitin-Ligase Funktion hat, besteht seine Hauptaufgabe in der Ubiquitylierung von anderen Proteinen. Welche das genau sind, ist immer noch Bestandteil aktueller Forschung.

\section{$1.5 \quad$ Ziele der Arbeit}

Die dieser Arbeit zugrunde liegenden Publikationen gehen den Zusammenhängen zwischen mitochondrialen Fehlfunktionen und dem Auftreten von neurodegenerativen Krankheiten, insbesondere Parkinson, nach. Da bei Menschen die Krankheit erst im hohen Alter auftritt und so viele Fälle ohne direkte Ursache vorliegen, liegt der Schluss nahe, dass neben genetischen Ursachen auch Umweltfaktoren eine größere Rolle spielen. Um dies zu analysieren, wurden experimentell verschiedene Stressoren eingesetzt.

Insgesamt wurden folgende Aspekte untersucht:

I. Welche Auswirkungen hat das Fehlen von PINK1 auf die Zelle? Gibt es einen Biomarker, der mit höherem Alter immer stärker verändert ist?

II. Welchen Einfluss haben Umweltfaktoren wie veränderte Eisen-Exposition auf die Zelle und was verändert sich beim Fehlen von PINK1?

III. Wie können mitochondriale Fehlfunktionen präferentiell das Nervensystem betreffen, wenn es nicht um respiratorische Insuffizienz geht?

\subsection{Ergebnisse}

I. Veröffentlichung: PINK1 und Neuroinflammation

In Torres-Odio/Key et al. 2017 wurde nach molekularen Biomarkern gesucht, wodurch Parkinson präsymptomatisch erkannt und die Progression der Erkrankung eingeschätzt werden kann. Hierzu wurden Kleinhirne von Pink $1^{-/-}$Mäusen in drei 
verschiedenen Altersstufen sowie primäre Neuronen und Fibroblasten von MausEmbryonen mit Pink1-Mutation untersucht. In einem weiteren Schritt wurden die Ergebnisse in einer humanen neuronalen Zelllinie mit PINK1-Knockdown nach der Exposition mit verschiedenen Stressoren validiert. Im letzten Schritt wurden humane Haut-Fibroblasten-Zelllinien von Patienten mit PINK1-abhängigem PD analysiert. Die Ergebnisse zeigen eindrücklich, dass nicht ein einzelner Faktor immer stärker verändert war, sondern, dass immer mehr Faktoren und daher auch eine steigende Zahl an Signalwege mit höherem Alter beteiligt waren.

Diese Anzahl an Dysregulationen in unterschiedlichen Signalkaskaden der Zelle betraf in jungen Tieren die nukleäre Spleiß-Maschinerie. Bei älteren Mäusen fielen auch der Ubiquitin-abhängige Proteinabbau und die Protein-Prozessierung am Endoplasmatischen Retikulum als abnormal auf. In Geweben von älteren Tieren (18 Monate) waren zudem Faktoren auffällig, die zu einer veränderten Neurotransmission führen. Weitere veränderte Signalwege betrafen Mitophagie und antimikrobielle Signalkaskaden. Interessanterweise traten erniedrigte Faktoren von inflammatorischen Signalwegen in den Vordergrund. Besonders verändert waren Faktoren, die mit der Erkennung und Verarbeitung von zellfremden Nukleinsäuren assoziiert sind. Weitere Experimente zur Bestätigung der Befunde wurden unter dem Einsatz von verschiedenen Stressoren wie Alpha-Synuklein-Überexpression, Nährstoffentzug oder der Exposition mit dem mitochondrialen Entkoppler FCCP und dem RNA-Analogon Poly(l:C) durchgeführt. Hierbei wurde deutlich, dass obige Befunde unter Stressbedingungen verstärkt waren.

Unsere Ergebnisse lassen den Schluss zu, dass das angeborene Immunsystem in Neuronen durch eine PINK1-assoziierte mitochondriale Störung aktiviert wird. In Pink1-- Mäusen konnten auch in hohen Altersstufen bis 24 Monate weder ein Verlust an Neuronen noch motorische Defizite nachgewiesen werden. Dies deutet darauf hin, dass die Entzündungsreaktionen des angeborenen Immunsystems in Neuronen deutlich früher eintraten als ein Verlust von Nervenzellen. Diese Studie erlaubt erstmalig Rückschlüsse auf zeitlich relevante Änderungen im Verlauf von Parkinson. Zudem konnte die zytosolische Rolle von PINK1 für antimikrobielle Signalwege identifiziert werden, in der PINK1 dann zur Aktivierung des angeborenen Immunsystems führt. 
II. Veröffentlichung: PINK1 und Eisenmetabolismus

Nachdem Stressoren eine wichtige Rolle bei Parkinson spielen, wurde in der Publikation Key et al. 2020 Eisen als ein weiterer im täglichen Leben vorkommender Stressor verwendet. Es wurden embryonale Maus-Fibroblasten mit Pink1-Verlust unter verschiedenen Eisenkonzentrationen kultiviert und umfassend analysiert. Da Eisen essenziell für Mitochondrien und deren Funktion ist, war besonders der Zusammenhang zwischen mitochondrialer Fehlfunktion und Eisen als Stressor interessant. Zudem werden Eisen-Chelatoren als medikamentöse Therapie bei Parkinson bereits in der Klinik diskutiert, sodass die molekularen Befunde große Relevanz für Patienten haben. Die Ergebnisse zeigen, dass unter niedrigen Eisenspiegeln Proteine, die am Nukleotid-Stoffwechsel beteiligt sind, vermindert waren. Außerdem deutlich reduziert waren Faktoren, die Eisen-Schwefel-Cluster als Cofaktoren haben und die wichtig für die Nukleotid-Qualitätskontrolle sind und somit mit Zellwachstum und -Überleben zusammenhängen. Insgesamt führt das Fehlen von Eisen zu einer Induktion von PINK1 und PARKIN, was auf verstärkte Mitophagie hindeutet.

Die Effekte von hohen Eisenkonzentrationen waren beim Fehlen von PINK1 deutlich verstärkt, was zu einer starken Expression des ribosomalen Faktors Abce1 und von Ferritin, der Speicherform von Eisen, führte. Unsere Studie ist die erste, die systematisch Faktoren des Eisenstoffwechsels nach Eisenüberladung und Eisenmangel im Zusammenhang mit Parkinson-Mutationen untersucht. Insgesamt geht daraus hervor, dass die mitochondriale Eisen-Schwefel-Cluster Biogenese und die post-transkriptionelle Eisenregulation entscheidend für die Pathogenese von Parkinson, bzw. das gesunde Fortbestehen einer Zelle und letztlich auch eines Organismus sind.

III. Veröffentlichung: PARKIN und seine Substrate - Relevanz für Neuronen

In der Publikation Key et al. 2019 wurde erstmalig das Gesamt-Ubiquitylom aus Gehirnen von gealterten Parkin-knockout (KO) Mäusen erhoben und analysiert. Durch die Identifizierung der Ubiquitylierungs-Substrate von PARKIN und der posttranskriptionellen Modifikationen wurde der Frage nachgegangen, wie mitochondriale Fehlfunktionen vorranging das Nervensystem betreffen können, wenn keine primäre 
respiratorische Insuffizienz vorliegt. Zudem wurde der funktionellen Bedeutung des veränderten Ubiquityloms für das Gehirn nachgegangen. Die molekularen Ergebnisse wurden durch elektrophysiologische Untersuchungen und Zellkultur-Arbeiten an embryonalen Maus-Fibroblasten ergänzt. Der Fokus lag hier auf Neuronenspezifischen Faktoren, die für axonale Erregbarkeit zuständig sind. Die Ergebnisse bestätigten, dass das am stärksten ubiquitylierte Substrat von PARKIN ein Transmembranfaktor der mitochondrialen Außenmembran, namens PORIN3 (VDAC3), ist. Dieser ist für die zelluläre Calcium-Homöostase von Bedeutung. Als weitere Substrate zeigten sich auch andere Faktoren, die den Calciumfluss in der Zelle regulieren. Dies betraf insbesondere den neuronalen Calciumsensor Hippocalcin, welcher auf Proteinebene erhöht und dessen Ubiquitylierung erniedrigt war, sowie Gna11, was durch transkriptionelle Erhöhung auffiel. Ebenfalls erwähnenswert ist die Erhöhung des Proteinspiegels der Kalium-Natrium-Pumpe ATP1A2, welche auch im Calciumhaushalt wichtig ist. Weitere „patch- clamp“ Experimente in Gehirnen von gealterten Parkin ${ }^{-/}-\mathrm{KO}$ Mäusen ergaben, dass in Neuronen im Locus coeruleus die Geschwindigkeit der spontanen Taktgeber erhöht, dass die langsame Nachhyperpolarisation reduziert und dass die Dauer der Aktionspotentiale erniedrigt war, ohne Veränderung der Kaliumkanal-Ströme. Die erhöhte neuronale Erregbarkeit und veränderte Nachhyperpolarisation könnten spezifisch für Parkinson sein, da ähnliche Beobachtungen auch in anderen Mausmodellen wie Pink1/-- und AlphaSynunklein-Überexpression gemacht wurden.

Insgesamt geht aus den 3 Studien hervor, dass mitochondriale Fehlfunktionen bei dauerhaftem Bestehen weitreichende Folgen für die Gesundheit des Nervensystems haben kann, denn auch kleine Veränderungen, seien es durch Mutationen oder Umweltfaktoren wie Eisen, können in einer so großen Lebensspanne wie der des Menschen über Krankheit oder Gesundheit entscheiden! 


\section{Summary}

\subsection{Mitochondria and their importance for the cell}

Since their discovery in 1886 mitochondria remain a mystery within the cell. Later they were described as "powerhouses of the cell" because they are the location of the respiratory chain and via the subsequent oxidative phosphorylation, they can generate the molecule ATP, the source of energy for the cell. Beyond that, mitochondria are involved in many other metabolic pathways within the cell. They are also involved in various other intracellular pathways, such as in the regulation of calcium homeostasis. Their double membranes also serve as platforms for cellular reactions, in particular immunologic pathways. Also, intracellular iron metabolism is closely linked to mitochondria. Iron can be stored in these organelles in a special ferritin form and both, heme and iron-sulfur-cluster (ISC) are synthesized within mitochondria, without which the cell could not survive.

Mitochondria occupy a special role within the cell since after the endosymbiotic theory [2] they are believed to stem from the symbiosis of a eucaryotic cell with a procaryote. Mitochondria have a unique structure because their double membrane encloses their own genome. Besides the nuclear DNA (deoxyribonucleic acid), the mitochondrial DNA encodes for mitochondrial proteins and is thus highly important for the survival of the cell. Due to their origin from bacteria, mitochondria are well tolerated by the cell but also have to be meticulously controlled. Any dysfunction can put the cell into an alarm state, in which it reacts as if a microorganism has invaded and has to be eliminated. One important mechanism for the selective degradation of mitochondria is mitophagy. In this process, damaged or malfunctioning mitochondria are marked and degraded via the autophago-lysosomal pathway.

\subsection{Mitochondrial dysfunction}

The importance of mitochondria for the health of cells, organs, and organisms can well be seen in the manifold of diseases where mitochondrial dysfunction can play a role. These diseases can affect almost every part of the body. One would automatically assume that mitochondrial dysfunction results in a respiratory problem with a subsequent energy deficit. The primary mitochondrial diseases include for example mitochondrial myopathy, Leigh syndrome, or MELAS syndrome all of which can be 
attributed to a mutation in the mitochondrial or nuclear genome. However, other diseases with an underlying mitochondrial dysfunction are much more frequent and better known. These are for example Diabetes mellitus, psychological illnesses like schizophrenia, and cancer. Mitochondrial health is also of great importance for neurodegenerative diseases such as Alzheimer's disease and Parkinson's disease. This thesis discusses the associations between mitochondrial health and the pathogenesis of Parkinson's disease.

\subsection{Parkinson's disease}

Parkinson's disease (PD) as the second most frequent neurodegenerative disease is of great importance in both clinics and research. The main signs include rigidity and bradykinesia, as well as tremor and postural instability. In postmortem patient brains, there is a loss of dopaminergic neurons in the substantia nigra resulting in the first two signs. Additionally, there are intracellular inclusions - called Lewy bodies - in the affected neurons, consisting of alpha-synuclein and other proteins such as ubiquitin. Furthermore, the iron metabolism in the brains of Parkinson's patients is disturbed and there are iron accumulations, especially in the midbrain.

The causes of PD are not yet fully understood. Most of the cases are sporadic with unknown causes and only a small percentage is the consequence of a genetic mutation. The most frequent mutations affect the genes encoding alpha-synuclein, PINK1, and PARKIN.

\section{$2.4 \quad$ PINK1 and PARKIN}

The serine-threonine kinase PINK1 and the E3-ubiquitin-ligase PARKIN are two proteins that act in stress situations to orchestrate the degradation of old or malfunctioning mitochondria at the outer mitochondrial membrane. In the case of nonfunctional organelles, PINK1 translocates from the cytoplasm to the mitochondrial outer membrane and recruits PARKIN resulting in the degradation of the affected organelles. Since PARKIN has E3-ubiquitin-ligase function, its main task is the ubiquitination of other proteins. Most of the substrates of PARKIN, however, are still under research. 


\section{$2.5 \quad$ Objectives}

The publications on which this thesis is based on are focused on the associations between mitochondrial dysfunctions and the pathogenesis of neurodegenerative diseases, in particular PD. Since PD affects humans primarily at higher ages and the causes are mostly unknown, it is likely that also environmental factors play important roles. To further analyze this, we used several stressors in our experiments.

The following aspects were analyzed:

I. What are the consequences of the loss of PINK1 for the cell? Is there a specific biomarker that gets more dysregulated with increasing age?

II. What are the consequences of environmental factors such as altered iron levels for the cell and how does this correlate with the loss of PINK1?

III. How can mitochondrial dysfunctions preferentially affect the nervous system if the underlying problem is not a respiratory problem?

\section{$2.6 \quad$ Results}

\section{Publication: PINK1 and neuroinflammation}

In Torres-Odio/Key et al. 2017, we looked for biomarkers that would allow the presymptomatic diagnosis and the progression of PD. We investigated cerebella of Pink $1^{-1-}$ mice at three different ages, as well as primary neurons and mouse embryonic fibroblasts with Pink1 loss. In a further step, the results were validated in a human neuronal cell line with PINK1-knockdown after the exposition with several stressors. In the last step, human skin fibroblasts from patients with PINK1-triggered PD were analyzed. The results show impressively that not a single factor is altered with even stronger fold-changes, but instead an increasing number of factors and thus a higher number of pathways gets involved with higher age.

These dysregulations in different signal cascades first affected the splicing machinery in young animals. In older mice, alterations of protein processing, both ubiquitindependent and at the endoplasmic reticulum (ER) became apparent. In the tissues of aged mice (18 months) we additionally saw factors changed that lead to altered neurotransmission. Further altered pathways included mitophagy and antimicrobial cascades. Interestingly, especially inflammatory pathways were significantly 
decreased. Among them, the highest alterations could be seen for factors relevant for the recognition and processing of foreign nucleic acids. In further experiments, the findings were validated upon the various stressors, such as starvation, alpha-synuclein (SNCA) overexpression, or exposure with the mitochondrial uncoupler FCCP or the RNA-analogue Poly(l:C). It was shown that the previous results were increased under stress conditions.

Our results lead to the conclusion that the innate immune system gets activated in neurons via a PINK1-associated mitochondrial dysfunction. In Pink1/- mice neither neuronal loss nor motor deficits were detectable, even at very high ages of 24 months. This indicates that the inflammatory reaction of the innate immune system in neurons precedes the consequent loss of neurons. This study for the first time allows conclusions about the time-dependent alterations in the pathogenesis of PD. Additionally, we were able to identify the cytosolic role of PINK1 for antimicrobial pathways, given that its depletion activates the innate immune system.

\section{Publication: Pink1 and iron metabolism}

Since stressors are of great importance in the pathogenesis of PD, in the publication Key et al. 2020 we used iron as a stressor that is present in our everyday life. We used embryonic mouse fibroblasts with Pink1 mutation and stressed them with different iron concentrations before analyses. Since iron is essential for mitochondria and their functions, especially the connection between mitochondrial dysfunction and iron as a stressor was important. Also, iron chelators are discussed as medical therapy in PD, so the molecular findings have great relevance for patients.

Our results show that in the presence of low iron levels proteins were diminished that regulate nucleotide metabolism. Also, factors were reduced that have iron-sulfurcluster as co-factors and are relevant for nucleotide quality control, and thus, are important for the cell's growth and survival. Altogether, the loss of iron led to an induction of PINK1 and PARKIN, indicating increased mitophagy.

When PINK1 was absent and iron levels were increased, the aforementioned effects were remarkably stronger. The most relevant findings were an increase in the ribosomal recycling factor Abce1 and the iron storage protein ferritin. Our study is the first one to systematically analyze factors of the iron metabolism after iron overload 
and iron depletion in the context of PD. Overall, the results show that the mitochondrial iron-sulfur-cluster biogenesis and the posttranscriptional iron regulation are essential for the pathogenesis of PD and the healthy presence of a cell and subsequently also for the health of an organism.

III. Publication: PARKIN and its substrates- relevance for neurons

The publication Key et al. 2019 for the first time analyzed the ubiquitylome of the brains of aged PARKIN-deficient mice. For the identification of the ubiquitylation substrates of PARKIN and the post-transcriptional modifications, we wanted to answer the question of how mitochondrial dysfunction can preferentially affect the nervous system if the underlying problem is not a respiratory failure. Also, we aimed to investigate the functional consequences for the brain. The most relevant molecular findings were complemented by electrophysiological techniques as well as cell culture experiments with embryonal mouse fibroblasts. We focused on neuron-specific factors that are relevant for axonal excitability. Our results confirmed that the strongest ubiquitylated substrate of PARKIN is the transmembrane factor of the mitochondrial outer membrane, PORIN3 (VDAC3). It is relevant for cellular calcium homeostasis. Also other substrates we identified are involved in the calcium flux within the cell. To mention two more, the neuronal calcium sensor Hippocalcin was elevated on protein levels while its ubiquitylation was decreased and Gna11 was transcriptionally induced. Another interesting factor was the sodium-potassium-pump ATP1A2, which is also known for its role in calcium homeostasis. The patch-clamp results in the brains of aged PARKIN-deficient mice revealed that locus coeruleus neurons showed accelerated spontaneous pacemaker frequency, decreased slow afterhyperpolarization, and shortening of the duration of action potentials without changes of potassium currents. The increased neuronal excitability and altered afterhyperpolarization could be specific for PD, since similar observations had been made in other mouse models, such as Pink $1^{-/-}$and alpha-synuclein overexpression mice.

Altogether, the three studies show that if mitochondrial dysfunction persists chronically it can have wide-ranging effects on the health of the nervous system. During the long 
lifespan of a human, also small abnormalities, such as mutations or environmental factors like iron can make the difference over health or disease! 


\section{Introduction}

New neurons can be generated in the body from neural stem cells. This so-called neurogenesis is mostly occurring during embryonic development and declines during childhood. In adults, however, there are only very distinct regions where neurogenesis can be observed. These include the subventricular zone along the walls of the lateral ventricles and the subgranular zone in the hippocampus. This implicates that once there is a loss of neurons in an adult human there is hardly any physiological replacement and the precious brain tissue is then lost. The two most common neurodegenerative diseases are Alzheimer's disease (AD) and Parkinson's disease (PD), both of which are associated with a loss of neurons [3]. Consequently, current research aims to regenerate the nervous tissue by the reprogramming of fibroblasts into induced pluripotent stem cells (iPSC). For PD, one therapeutical intervention includes the implantation of either reprogrammed neurons or embryonic stem (ES) cells into the putamen [3]. However, before the treatment of such a widespread and multi-factorially triggered disease comes into play, the basics of the pathogenesis are part of current research. Thus, to further understand factors and pathways that are involved in the development of the disease and to identify therapeutic options, the role of mitochondria in neurodegenerative diseases is crucial. Details on the connection between these organelles and PD will be discussed in more detail in a later paragraph.

\subsection{Mitochondria}

Mitochondria are double-membraned intracellular organelles that govern many functions in various cellular processes. Their origin is described in the endosymbiotic theory, as these important organelles are thought to stem from invading proteobacteria that once formed a symbiosis with eucaryotic cells. Mitochondria were later included as cellular organelles [4]. The structure of mitochondria is unique within the cell since they are the only organelle that possesses its own circular genome. In humans, the 37 genes encode for 13 proteins. These proteins are part of the enzyme complexes of the oxidative phosphorylation (OXPHOS) system and thus, mitochondrial DNA (mtDNA) is almost as important for the cell's survival as nuclear DNA. The DNA of mitochondria has features similar to bacterial DNA, both contain hypomethylated CpG motifs, which can activate innate immune pathways through Toll-like receptor 9 (TLR9) [5]. Like 
bacteria, mitochondrial proteins can have a formyl group attached to a methionine at their N-terminus, so if mitochondria are dysfunctional and leaky these proteins would be recognized by the innate immune system [6].

Mitochondria are involved in many different metabolic and signaling pathways. Their most obvious role lies in the production of energy in the form of ATP, providing the conditions for the respiratory chain to function. Apart from ATP, they are also the main source of NADPH, which is produced through the tricarboxylic acid cycle (TCA) and OXPHOS [7]. In the nervous system, this energy production is needed to establish appropriate electrochemical gradients and reliable synaptic transmission.

Mitochondria also function in the regulation of protein quality control. Their role in the mitochondrial unfolded protein response (UPRmt) was thoroughly described in $C$. elegans and is conserved until mammals [8].

Mitochondria also play important roles in the regulation of the intracellular calcium homeostasis by buffering these important molecules [9] and they also have a crucial impact on iron homeostasis. With their membranes, mitochondria provide platforms for signaling pathways, one important aspect lies in the regulation of innate immunity processes $[10,11]$. Another important function of mitochondria lies in the regulation of apoptosis [12]. Thus, it is highly logical that mitochondrial health is crucial for the survival of cells and organs with any malfunction leading to disastrous effects.

\subsection{Mitochondrial dysfunction}

The importance of mitochondria for the cell becomes obvious when considering their manifold functions. Consequently, a lack of mitochondrial fidelity through either mutations or exposure to environmental substances can have detrimental effects. In general, mitochondrial dysfunction is characterized by a loss of efficiency in the electron transport chain or of the OXPHOS system. Hence, mitochondrial dysfunction is closely related to a lack of energy, followed by increased reactive oxygen species (ROS). This implies that mitochondrial dysfunction results in a respiratory problem with a subsequent energy deficit. Regarding the diseases where mitochondrial dysfunction plays a role in, there are primary and secondary mitochondrial diseases. The primary mitochondrial disease (PMD) genes either encode OXPHOS proteins directly or indirectly by impacting the production of the complex machinery needed to run the 
OXPHOS process. PMD include for example Leigh syndrome, the most common pediatric presentation of mitochondrial disease with severe progressive encephalopathy, lactic acidosis, and stroke-like episodes [13]. Another example is MELAS syndrome, which is a mitochondrial encephalo-myopathy [14]. Both diseases are caused by mutations in genes coding for mitochondrial electron transport chain (ETC) proteins, in the nuclear of the mitochondrial genome. In secondary mitochondrial disease (SMD) affected genes neither play a role in the function nor in the production of OXPHOS proteins. However, other diseases with an underlying mitochondrial dysfunction are much more frequent and better known. These are for example Diabetes mellitus [15], psychological illnesses like schizophrenia [16], and cancer [17]. Another secondary mitochondrial disorder is Friedreich's Ataxia (FA), where Frataxin, a factor involved in iron metabolism, is affected [18].

The most frequent diseases that can be associated with malfunctioning mitochondria, however, are neurodegenerative diseases, in particular AD and PD [19]. Thus, to achieve treatment options for many patients, research with a focus on the connection between mitochondria and neurodegenerative diseases is needed. This thesis focuses on the role of dysfunctional mitochondria through either genetic causes or environmental triggers in the pathogenesis of PD.

\subsection{Parkinson's disease}

$P D$ is the second most common neurodegenerative disease after $A D$ in the aging population [20, 21]. PD is characterized by four cardinal signs - rigidity and bradykinesia, as well as tremor and postural instability. The first two signs can be attributed to brain pathophysiology where a loss of dopaminergic neurons in the substantia nigra pars compacta (SNpc) can be observed. In the affected neurons of PD patient brains, there are cytoplasmatic inclusions that are called Lewy bodies, which among other components contain alpha-synuclein and other proteins, such as ubiquitin.

Prior to the appearance of locomotor deficits, patients show various non-motor symptoms including sleep disturbances, hyposmia, constipation, and psychiatric changes $[22,23]$. These additional symptoms reflect the involvement of other areas of the nervous system, such as brainstem structures, the olfactory bulb, the 
gastrointestinal nervous system, the limbic system, and the locus coeruleus (LC) [24]. The majority of PD cases are sporadic with unknown causes, but in the past decades, several monogenic forms were identified that can be attributed to mutations in a single gene. These cases, however, only account for $3-5 \%$ of the sporadic cases [25]. The first gene, which was found to lead to an autosomal dominant form of PD, was alphasynuclein, described in 1997 [26].

Among the monogenic variants that lead to autosomal recessive PD, mutations in PINK1 or PARKIN (PRKN) genes are frequent causes of early-onset PD, and cases with these underlying mutations were called PARK6 and PARK2, respectively $[27,28]$.

\subsection{Mitochondrial quality control}

Mitochondria undergo various dynamic processes, including fusion and fission, mitogenesis, and their autophagic degradation, which is called mitophagy. To maintain a stable network, all these processes have to be in a homeostatic balance. To ensure mitochondrial fidelity and thus, to keep cells in a healthy state, cells have various control mechanisms. One way of degrading mitochondria as a whole when they are malfunctioning is via the PINK1/PARKIN-dependent mitophagy. The general concept of PINK1/PARKIN-dependent mitophagy is shown in Figure 2. 


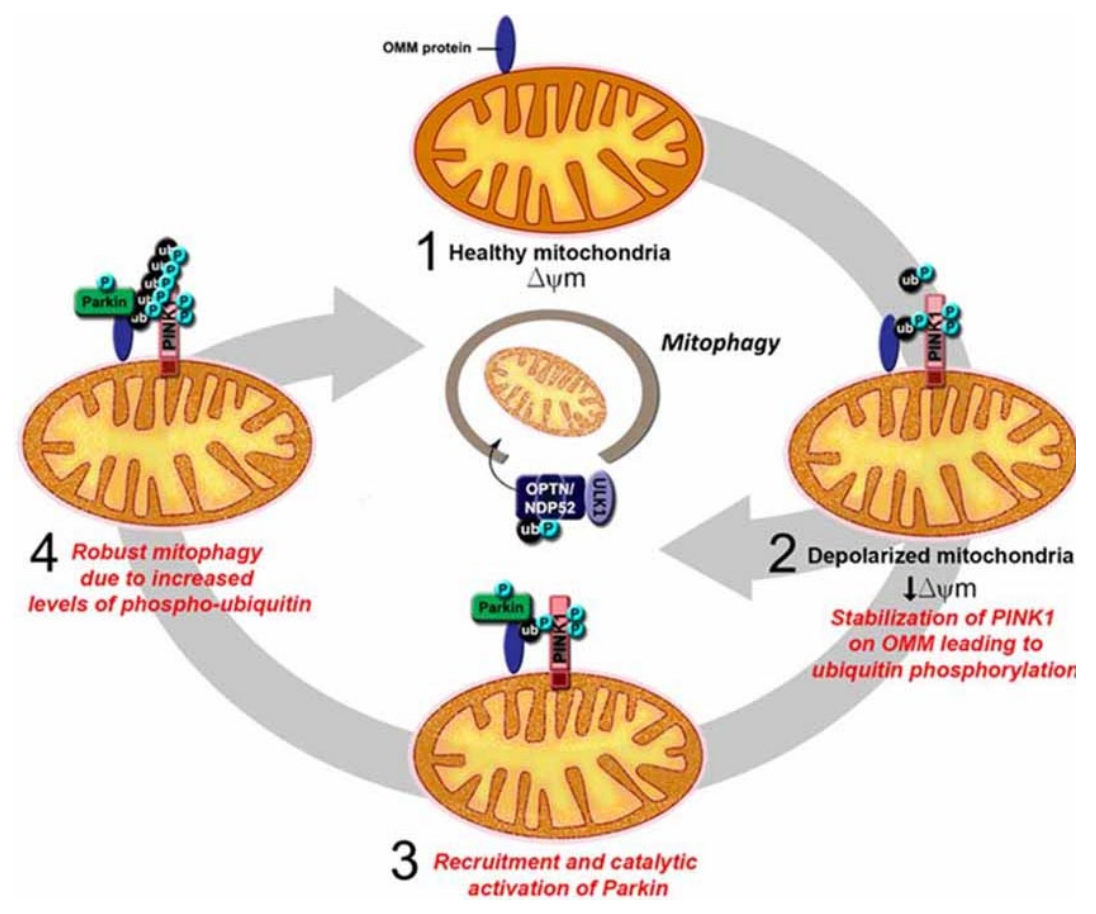

Figure 2: Scheme of PINK1/PARKIN regulated mitophagy. 1) Healthy mitochondria with stable membrane potential. 2) PINK1 accumulation on the outer mitochondrial membrane as a consequence of depolarization and subsequent phosphorylation of ubiquitin. 3) Recruitment of PARKIN and phosphorylation by PINK1. 4) Polyubiquitylation of mitochondrial substrates and thus, induction of mitophagy [29]

\subsubsection{PINK1}

PINK1 (PTEN induced kinase 1) is a serine-threonine kinase that is at the outer mitochondrial membrane. The full protein contains three domains: an N-terminal mitochondrial target sequence (MTS), a transmembrane domain, and a serinethreonine kinase domain [30]. Under physiological conditions with normal functioning mitochondria, the transmembrane domain of PINK1 gets imported into the mitochondrial intermembrane space, where it gets cleaved by a peptidase called PARL (Presenilins-associated rhomboid-like protein) and it gets degraded [31]. However, when mitochondria become dysfunctional and depolarize, PINK1 is not degraded, but instead will accumulate on the outer mitochondrial membrane (OMM). There, it recruits PARKIN from the cytosol to induce the autophago-lysosomal degradation of the dysfunctional organelles [32]. PINK1 is transcriptionally induced upon stress situations, especially nutrient starvation, and can thus be described as a stress response factor [33]. Mutations in PINK1 in respect to PD were first described in 2004 and as another novelty linked the pathogenesis of PD to mitochondrial health [27]. PARK6 is a recessively inherited, early-onset form of PD that can already become apparent at 20- 
50 years of age. Interestingly, the mouse model with a mutation in Pink1 leading to the absence of the protein shows no neurodegeneration, but progressive mitochondrial dysfunction, defects of core mitochondrial functions like ATP-generation and respiration, and consequently impaired neural activity [34]. To further understand the connection of PINK1-deficiency and the aforementioned symptoms, further research is needed and thus, this mouse model was used for in-depth analyses and further molecular characterization.

\subsubsection{PARKIN}

The PARK2 variant of PD is important since the underlying mutations in the PARKIN gene account for up to $50 \%$ of the autosomal recessive PD cases. The gene encodes for an E3-ubiquitin ligase, called PARKIN. Although localized in the cytosol, PARKIN is involved in the regulation of mitophagy during stress periods. In its inactivated state, PARKIN is in an auto-inhibited form. After re-localization to the outer membrane of depolarized mitochondria, it regulates the removal of the damaged organelle [35]. Since PARKIN has an E3-ubiquitin-ligase function, its main mechanism of action is via ubiquitylation of other proteins. Thus, to further understand the pathophysiology of PD, identifying its substrates is of crucial importance. To succeed in that, mouse models with the deletion of the Prkn gene and subsequent absence of PARKIN were generated and are the subject of recent research [36, 37]. These mice do not show robust parkinsonian symptoms and are thought to display inconsistent phenotypes [38-40]. Mitochondria are in close contact with the ER within a cell and together these organelles play important roles in calcium homeostasis. Thus, the association between PARKIN and Mitofusin-2 at the mitochondrion-associated ER membrane compartment was described before [41]. However, the impact of that on neurotransmission and brain function remains elusive and is part of this thesis.

\subsection{Iron metabolism}

Mitochondrial function is closely connected to environmental stress. In experiments, mitochondrial uncoupler are often used as stressors. Since in PD patients, the iron metabolism is disturbed and there are iron accumulations, especially in the midbrain 
[42], the consequent step is to assess the association of iron levels and PD in cell culture to get molecular insights.

Iron is an essential micronutrient for the cell and in its free form; iron can have toxic effects and can result in increased ROS production [43]. Consequently, various mechanisms have evolved to uptake, recycle and store iron while minimizing toxicity. To ensure cellular fidelity, iron is further utilized for heme and ISC, both processes being localized within and closely connected to mitochondria [44, 45]. Since ISC are crucial for cellular health, this synthesis must function well and depends on mitochondrial health. The general iron metabolism of a cell is depicted in Figure 3.

Generally, iron is either stored within the cell in the form of ferritin, utilized in mitochondria for the synthesis of heme and ISC, incorporated into iron proteins (for example involved in nucleotide surveillance) or exported from the cell. Since there are manifold pathways in which iron is involved, the impact of altered iron levels should not be underestimated. In this thesis, the impact of disturbed iron metabolism and its connection to insufficient mitophagy through the loss of PINK1 is discussed.

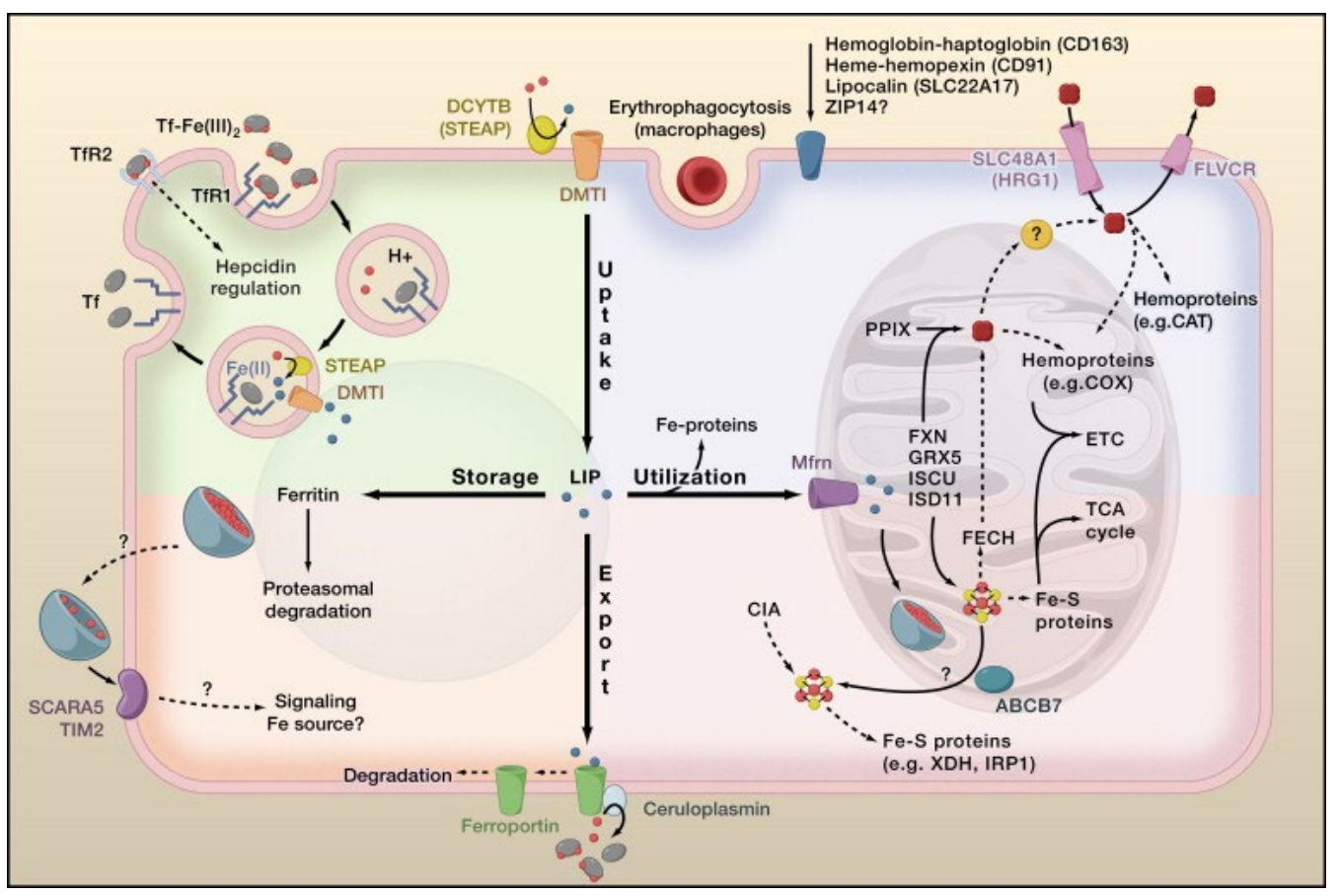

Figure 3: Iron metabolism in an exemplary cell. Iron is taken up at the plasma membrane by Transferrin receptor (TfR1) mediated endocytosis and reduced to $\mathrm{Fe}^{2+}$ in endosomes. This iron is then available to the cell from the labile iron pool (LIP). It is utilized in the cell for 1) incorporation into iron proteins, 2) transport to mitochondria for the insertion into heme and ISC or storage in the mitochondrial ferritin (Mfrn), 3) storage in the form of ferritin or 4) export from the cell. [12] 


\section{Aim of the thesis}

The publications on which this thesis is based on, deal with the associations between mitochondrial dysfunctions and the pathogenesis of neurodegenerative diseases, in particular PD. Since PD affects humans primarily at higher ages and the causes are mostly unknown, it is likely that also environmental factors play important roles. To further analyze this, we used several stressors in our experiments.

This thesis aims to answer the following questions:

I. What are the consequences of the loss of PINK1 for the cell? Is there a specific molecular biomarker that gets more dysregulated with increasing age?

II. What are the consequences of environmental factors such as altered iron levels for the cell and how does this correlate with the loss of PINK1?

III. How can mitochondrial dysfunctions preferentially affect the nervous system if the underlying problem is not a respiratory problem? 


\section{Results and discussion}

\subsection{Progression of pathology in PINK1-deficient tissues}

Since so many PD patients become diseased with unknown causes, basic research must take genetic mutations that lead to PD as a starting point. With PINK1 and PRKN mutations resulting in $\mathrm{PD}$, this links the role of mitochondria to the pathogenesis of PD. Research with human material, however, bears several limitations. Brain tissue from patients is only available post mortem, reflecting the last steps of disease and can potentially already show proteolytic degradation due to storage conditions. In contrast, peripheral cells from patients would not depict the neuronal state that can be found in the brain. Also, the heterogeneity of PD variants does not contribute to uniform and strategical experiments. Thus, one approach is the usage of mouse models. Current research with advanced technologies in gene editing allows analyses of chronic situations as they were found in patients.

Previously, in our laboratory, a mouse model with a loss of PINK1 protein was generated and characterized [34]. Now, to get a further understanding of the disease pathology and progression, there are two starting points. One is to investigate murine tissues with increasing ages to get insights into the first molecular changes and to potentially identify progression disease markers. Another strategy involves the analyses of different tissues from one species and validating the findings in other species, such as humans. Also taking the environmental factors of PD into account, the exposure of cells and cell lines with various stressors seems to hold promise.

In the publication Torres-Odio/Key 2017 [1], exactly these strategies were used to get deeper insights into the pathology of PD. One main focus was on the identification of disease progression biomarkers. For the first time, we globally analyzed brain tissues of mice from three different ages - 6 weeks, 6 months, and 18 months - by gathering their transcriptomes. Further experiments involved the analyses of murine primary neurons and mouse embryonic fibroblasts with Pink1 mutations. The findings were then validated in the human neuronal cell line SH-SY5Y with PINK1-knockdown after the exposure with several stressors. In the last step, human patient skin fibroblasts were analyzed to identify parallels and consistencies between species.

The comparison of the number of altered factors in the transcriptomes showed a drastic increase in numbers of dysregulations with increasing age - from 250, through 1300 , 
to 3500 factors, respectively. This already indicated that not one gene and a subsequent pathway is making the difference but rather the increasing number of factors that get altered with aging. Bioinformatic analyses by STRING protein-protein interaction and gene set enrichment (GSEA) software were conducted to also feature the description of the temporal order. With the STRING webserver, one gets insights not only into singular altered factors, but can also to recognize pathway changes. Going one step further, GSEA analyzes not only factors beyond a significance threshold but instead analyzes all factors genome-wide based on their fold-change, thus also allowing assertions about pathways that are subtly dysregulated. In mice already at the age of 6 weeks, the nuclear splice machinery was affected, especially around the splice factor Srsf10. This gene encodes for a stress response factor that regulates cellular response against DNA damage [46], already hinting towards an early occurrence of disturbed homeostasis in the absence of PINK1.

Older mice showed dysregulations in the pathways involved in ubiquitin-mediated proteolysis and protein processing at the ER. The first finding is in perfect agreement with the role of PINK1 as ubiquitin kinase Furthermore, its role at the ER puts PINK1 into a more global perspective with additional functions at the general protein processing and degradation rather than being limited to its role in mitophagy.

At the highest age of 18 months, we saw factors with changed expressions, which lead to altered neurotransmission. Further altered pathways included mitophagy and antimicrobial cascades. These included cascades, such as MAPK signaling and T-cell receptor signaling. Among these changes, the strongest alterations were seen for factors that are involved in the recognition and processing of foreign nucleic acids. The described alterations were confirmed with GSEA after STRING interaction analyses showing the robustness of the results.

Testing several other stress situations, it was seen that in double-mutant mice with Pink $1^{-1-}$ and SNCA overexpression as additional stress, there is a potentiated phenotype with the appearance of Lewy-like inclusion bodies and with lethality from the age of 14 months on [47]. These double-mutant mice were also characterized further on the molecular level via the generation of their SerThr-PhosphoProteome [48]. 
The dysregulation of anti-microbial defense key factors was reproducible under these conditions. Transferring the results to human neuronal cells also confirmed the transcriptional regulation of the anti-microbial response by PINK1 after acute starvation stress. Two more stressors were tested, FCCP as mitochondrial uncoupling agent to induce mitophagy and Poly $(I: C)$, which is a pathogenic RNA-analogue and mimics viral infection. Interestingly, in the absence of PINK1, the inflammatory response was diminished rather than increased compared to WT animals. This hints towards PINK1 acting as an enhancer of the inflammatory pathways, in particular RNA-sensors. Remarkable alterations could be seen especially for the mitochondrial antiviral signaling protein MAVS, the pathogenic dsRNA-sensors IFIT1, IFIT3 and DDX58, as well as for the viral replication suppressing factor RSAD2 [10, 49].

Our results lead to the conclusion that the innate immune system gets activated in neurons via a PINK1-associated mitochondrial dysfunction.

In general, neuroinflammation is described as a prominent microglial reactivity and activation of innate immune response pathways [50]. Previously, neuroinflammation had been described in connection to PD [51,52] and was thought to be a consequence of neuronal loss [53]. In contrast to that finding, in Pink 1-- mice, there were neither neuronal loss nor motor deficits detectable, also at very high ages of 24 months [34]. This indicates that the inflammatory reaction of the innate immune system in the brain precedes the loss of neurons. Our study for the first time allows conclusions about the time-dependent alterations in the pathogenesis of PD. Additionally, we were able to identify the cytosolic role of PINK1 for antimicrobial pathways where its depletion activates the innate immune system. This was also supported by a study linking PARKIN, which functions together with PINK1 in mitophagy, to the resistance against intracellular pathogens [54]. Further support was shown in another publication showing that PINK1 and PARKIN repress mitochondrial antigen presentation through the formation of mitochondrial-derived vesicles [55] and also, PARKIN was shown to regulate the modulation of the NLRP3 inflammasome [56].

Foreign or mislocalized endogenous DNA has long been known to stimulate innate immunity [57]. In the past years, evidence has accumulated that dysfunctional mitochondria can release these molecules, so-called DAMPs (damage-associated molecular patterns), such as mitochondrial DNA and RNA fragments, which have bacterial features, like hypomethylation [58]. The seemingly foreign nucleic acids get 
recognized by cytosolic sensors and activate downstream signaling pathways which induce an inflammatory state and can result in apoptosis. One mechanism is the cGASSTING pathway. The cytosolic molecule cGAS is a sensor of nucleic acids, releases cGMP and this activates STING, the central regulator of the type I interferon response to cytosolic DNA [59]. This pathway was identified as a regulator of microglial activity and neuroinflammation. Other pathways that aim to defend the cell against invading microorganisms include the recognition of cytosolic viral by the helicase DDX58, also known as RIG-I. This triggers the dimerization of MAVS at the outer mitochondrial membrane. Subsequently, transcription factors of the interferon response factor (IRF) family (IRF3, IRF7) translocate to the nucleus and trigger the induction of effector molecules such as IFIT1, IFIT3, and RSAD2, which then inhibit viral replication [59]. Figure 4 shows the findings of mitochondria-associated innate immune pathways in human neuronal cells with a knockdown of PINK1.

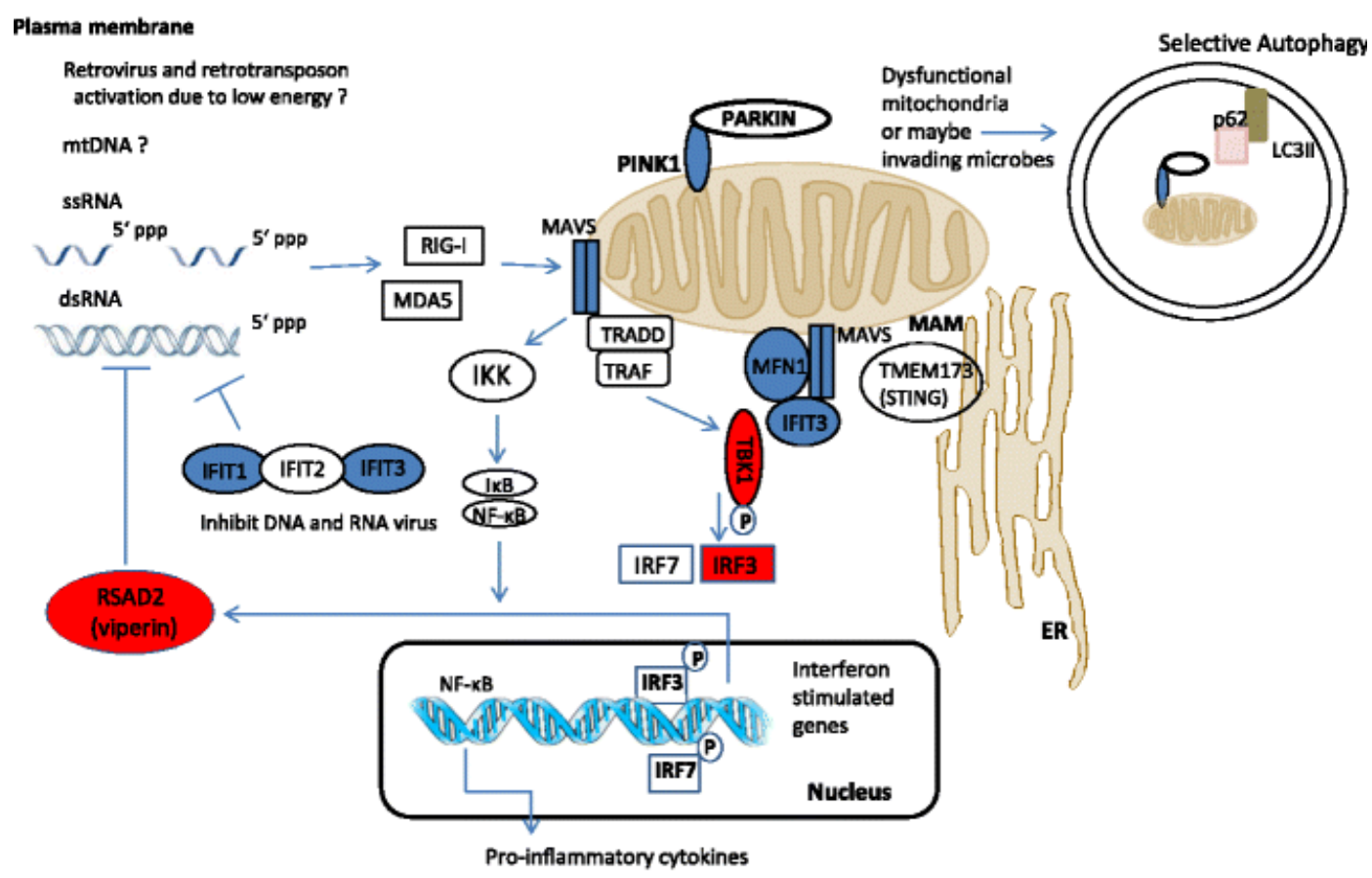

Figure 4: Scheme of PINK1-signaling in innate immunity. Systematic assessment of relevant factors in innate immunity signaling. The scheme shows transcriptional responses in the absence of human PINK1 in the neuronal cell line SH-SY5Y. Blue color indicates PINK1-dependent downregulation, red color transcriptional induction [1].

Similar, even stronger, findings of inflammatory phenotypes were reported in another mouse model with a form of mitochondrial dysfunction, which is the absence of the mitochondrial matrix protease CLPP (caseinolytic mitochondrial matrix peptidase proteolytic subunit) [60]. Patients with mutations in CLPP develop a disease called 
Perrault syndrome, characterized by early-onset infertility, sensorineural hearing loss, growth retardation, and cerebellar ataxia [61].

Our findings were also later confirmed in a study [62], proving a strong inflammatory phenotype in both $\mathrm{Prkn}^{-/-}$and Pink1-/- mice after exhaustive exercise or in Prkn ${ }^{-/}$ mutator mice, which accumulate mtDNA mutations. The inflammation could be rescued in either case by the loss of STING [63]. This shows the importance of innate immune reactions in connection to neurodegeneration. Additionally, mtDNA alterations were reported in the context of PD before [64].

In summary, the linkage of PINK1 to neuroinflammation, together with the documentation of a temporal scenario in this variant of PD emphasized the role of mitochondria and their integrity and function not only for Pink1 mutations but also for $P D$ in general. 


\subsection{PINK1 and iron metabolism}

Mitochondrial fidelity is closely connected to environmental stress. Stressors, again, are also important in the pathogenesis of PD. This connection has been known for a long time, given that the application of the illicit drug and neurotoxin MPTP results in the generation of parkinsonian symptoms in mammals [65-67]. After administration MPTP is converted into $\mathrm{MPP}^{+}$within the body, then gets imported into dopaminergic cells in the substantia nigra, and there interferes with complex I of the respiratory chain $[68,69]$. As a result, there is a loss of dopaminergic cells. After chronic administration, autopsies revealed Lewy body-like aggregates. MPTP has been used as a stressor in monkeys [70] and mice [71] to mimic parkinsonian phenotypes. To trigger malfunctioning mitochondria, in research, the uncoupling agents CCCP or FCCP [72] often come to use, which destroy the mitochondrial proton gradient and can elicit apoptosis. However, this scenario mainly reflects designed experiments and does not depict everyday stress conditions. We wanted to focus on stressors that are more likely to be present in a patient's everyday life and can thus give a more realistic picture. When post mortem brain samples of PD patients are analyzed, there are iron accumulations in the brain, especially in the midbrain [42]. Additionally, the entire iron metabolism is disturbed [73]. As mentioned before, iron utilization within the cell is closely connected to mitochondria as they are a place of iron storage. Even more importantly, they are the place of heme and ISC production, both of which are crucial for the cellular fidelity in various pathways. Apart from all the benefits, iron can also be toxic foir the cell as it can generate noxious hydroxyl radicals through the Fenton reaction [74]. This ROS production can result in a cell death called ferroptosis [75].

Consequently, the connection between malfunctioning mitochondria and different iron concentrations is of great interest. In the past, this has not been analyzed systematically, so we undertook the first compound analyses of this topic. Nevertheless, iron chelators are discussed for the treatment of PD [76] and they are already used in clinical trials. This makes it even more important to unravel the underlying molecular basics and pathways.

The study Key et al. 2020 [77] used mouse embryonic fibroblasts (MEF) with Pink1 mutation and subsequent knockout of the protein with matched wildtype cells and exposed them to different levels of iron contents in the cell culture medium. Iron overload was administered by the addition of ferric ammonium citrate (FAC) and for 
iron depletion, two different iron chelators were used, deferoxamine (DFO) and 2,2'-bipyridyl (22BP). To analyze all general alterations in various pathways, we examined the global proteome of MEF after the exposure to different iron conditions and validated highly relevant findings at transcript and protein levels via RT-qPCRs and quantitative immunoblots. This study is the first one to give a global overview of physiological regulations of pathways related to iron, ISC, and heme homeostasis with respect to mitochondria.

In general, the comparison of iron overload versus iron depletion showed that high iron levels resulted in fewer alterations than iron depletion. This can be attributed to the already considerable high iron levels that are present in cell culture medium in general and thus, an even higher concentration would not lead to such pronounced effects as efficient iron chelation.

The more detailed analyses of pathways altered after iron overload, identified enriched molecules involved in iron-binding, mitochondria, vesicles, and autophagy and diminished abundance of molecules involved in glycolysis and metabolic pathways. Comparing factors that showed converse regulation after iron overload versus iron depletion, we could identify the mitochondrial lipid transporter CPT1A, the matrix metalloprotease MMP14, the purine degradation enzyme XDH and the glycogenmobilizing factor PYGL. The heme-degrading factor HMOX1 and the nuclear transcription factor CREG1 showed upregulations upon both treatments.

In total, we could identify Pink1-dependent effects on

- 8 iron/heme/ISC factors, including FECH (Ferrochelatase) and GLRX5

- 8 unspecific mitochondrial factors, including MRPS35 and MRPS36

- 6 autophagy factors, including MAP1LC3A and BECN1

- 5 lysosomal factors, including LIPA

- 5 RNA splicing and surveillance modulators, including SRSF10

- 5 DNA repair factors, including RRM1 and CREBBP

- 4 innate immunity factors, including MYD88. 
These proteins very well reflect the known functions of PINK1 in mitochondrial degradation via the autophago-lysosomal pathway and underline the iron accumulation in Pink1-mutant brains.

Iron depletion led to the upregulation of beta-synuclein (SNCB), which is the alphasynuclein antagonist and interestingly, has ferroxidase properties.

The mRNAs of Pink1 and Parkin were both induced after low iron levels, indicating increased mitophagy and possibly ensuring cellular protection, which would be very important for the health of neurons in PD. This goes well together with the observations that iron shortage induces mitophagy in C. elegans [78].

Also, $i$ the presence of low iron levels resulted in the decreased abundances of proteins that are relevant for nucleotide synthesis and repair, such as $\mathrm{XDH}$ and the ferritin homolog RRM2. Both factors have iron-binding capacities, and their function is relevant for the maintenance of nuclear DNA. Especially RRM2 is of great interest because of its ferritin homology while it has functions in nucleotide synthesis.

Besides, there was a decrease of ISC-binding factors that also have functions in the surveillance of nucleotides, one example is the DNA glycosylase NTHL1. Alterations in these factors were affecting several subcellular compartments, including the cytosol, mitochondria, and nucleus. Dysregulations in these important proteins can affect the cellular growth and survival. One essential ISC-biogenesis factor, GLRX5, was even diminished under untreated conditions in WT versus Pink $1^{-/-}$fibroblasts at the protein level.

The most relevant finding was the decreased mRNA of ISC-containing factor ABCE1 under untreated conditions. ABCE1 is a highly conserved protein and it plays a role in translation initiation and ribosome recycling [79]. It was reported earlier, that in D. melanogaster mitophagy is regulated in a PINK1-dependent manner via ABCE1 [80], and the homozygous deletion of this protein in flies results in early lethality [81]. Our study now gives first evidence that also in mammals there is a PINK1-dependent mechanism regulating translational quality control by ABCE1. In general, the activity of several iron-regulatory proteins depends on the stability of their ISC, which are generated in mitochondria. This is especially important for IPR1/ACO1 and IRP2/IREB2 [82], which exert important iron-regulatory roles. 
Interestingly, in Pink1-- cells after FAC treatment the mRNA levels of ferritin heavy (Fth1) and light chain (Ftl1) showed hyper-reactive inductions in the absence of PINK1, whereas the protein levels remained stable. These observations might be due to posttranscriptional modifications caused by limited ISC availability or quality.

In search of the underlying molecular pathways that are changed, we analyzed several groups of targets of transcription factors to see if there was one transcription factor regulating the responses. NRF2 (nuclear factor, erythroid 2 like 2) regulates the antioxidant response within the cell. Interestingly, several of the targets of NRF2 showed altered transcriptional expressions, namely Fth1, Ft/1, Hmox1, and Nqo1. Other factors that we saw altered also have binding sites for NRF2. This makes the antioxidant NRF2-dependent cellular response pathway an interesting candidate as the underlying molecular mechanism. The connection between NRF2 and PINK1 had been reported before, with NRF2 regulating Pink1 expression under oxidative stress conditions [83]. However, other targets of NRF2 did not show transcriptional alterations, so there must be additional mechanisms involved. 


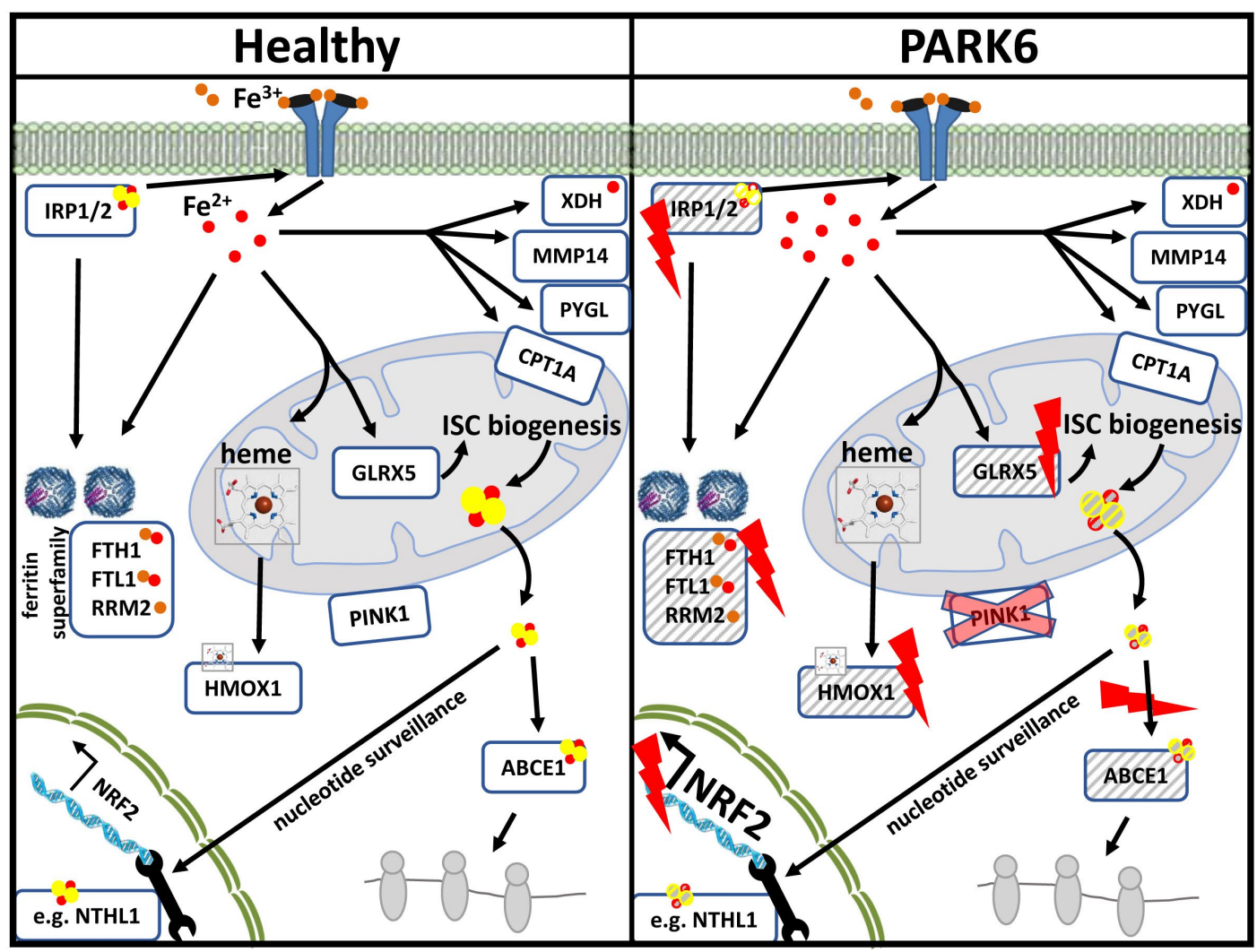

Figure 5: Summary of the relevant findings of iron levels in dependency on PINK1. The left side shows the healthy condition, the right side shows the murine model of PARK6 with a mutation in Pink1 and the changes seen after manipulations of extracellular iron conditions. Further explanation is given in the main text [77].

The most relevant findings, such as Abce1 and ferritin hyper-responsiveness were then validated in fibroblasts from PARK6 patients. The experiments showed consistency between mouse and human cells, again indicating abnormal iron homeostasis as well as consequences by insufficient ISC quality or availability.

Our data hint towards an inefficient iron usage in the mitochondria of PINK1-deficient cells, which would then be followed by increased iron import into the cell to maintain nucleotide quality control via ISC-associated proteins. The numerous cellular adaptations in response to lowered iron levels both, on protein and transcript levels, make clear that the iron concentrations have a high impact on various pathways. Thus, our results show that long-term treatment of patients with iron chelators is likely to have numerous side effects and should thus be done with care. The limited transcriptional abundance of those several nucleotide surveillance factors might contribute to the known instability of the mitochondrial genome in PD [84], a phenomenon that could then be enhanced after iron chelation therapy. Our data also gives an explanation why 
there is acutely improved motor performance in PINK1-mutant flies after iron supplementation [85].

Of course, the results presented here were collected in peripheral cells of mice and some results were then validated in patient skin fibroblasts. The impact of the findings has to be tested again in neuronal cells and potentially in mouse brains, for example with the help of organotypic slice cultures (OTC). Also, our experimental setup only covered short-term effects between 16-48 h. The long-term consequences of altered iron levels, especially iron deprivation have to be the subject of future research.

Our study is the first one to systematically analyze factors of the iron metabolism after iron overload and iron depletion in the context of PD. Overall, the results show that the mitochondrial ISC biogenesis and the post-transcriptional iron regulation are essential for the pathogenesis of PD and the healthy presence of a cell, and subsequently also for the health of an organism.

Interestingly, mice lacking the mitochondrial matrix peptidase CLPP were described to have increased circulating iron levels and increased hemoglobin levels [86], according to the international mouse phenotyping consortium (IMPC). Furthermore, these mice, representing a robust model of the human Perrault syndrome, have decreased body weight and increased levels of inflammatory factors [60]. The underlying molecular pathways are under current research but implicate that there is also a connection between mitochondrial dysfunction and iron/heme regulation. These observations are already apparent in unstressed conditions due to the more severe affection of mitochondria than in PINK1 deficiency. In additional manuscripts that go beyond the scope of this thesis, the impact of CLPP deficiency on mitochondrial functions and innate immunity activation is being analyzed $[87,88]$. CLPP is one of two mitochondrial matrix proteases, the other one is LONP1 (Lon peptidase). The latter one was also characterized and the proteomic effects of the loss of CLPP and LONP1 were compared [89]. CLPP was found to be important for mitoribosome assembly and translation fidelity. In comparison to that, we also analyzed mutants that serve as modulators of the cytosolic ribosomal translation [90].

It was recently shown that there is also a connection between iron homeostasis and age [91]. A study demonstrated that muscles of older people have higher abundances of labile iron content, higher levels of mitoferrin and frataxin, and increased mtDNA 
relative abundances. Besides, patients showed higher levels of PINK1. This indicates that the cellular and mitochondrial iron homeostasis is dysregulated in the aged group, linking the findings of our systematic analyses with the age-associated disease PD. The upregulation of PINK1 in aged people well explains the hyper-sensitivity we saw in cells with Pink1 mutations. It was shown that in neurons lacking PINK1 there was mitochondrial compensation for the loss of surveillance. The reduced mitochondrial membrane potential and mitochondrial aconitase could not be compensated for and triggered a metabolic rewiring. It might well be that the underlying factor for the mitochondrial aconitase deficiency is also connected to iron metabolism [92]. 


\subsection{PARKIN and its substrates - relevance for neurons}

As seen before, mitochondrial health can decide on the development of diseases, especially on age-associated diseases, such as PD. Since the main function of mitochondria in the brain probably lies in energy production, the first consequence of a malfunctioning mitochondrion would be the loss of its respiratory capacity. Consequently, such a cell would die from the loss of energy and the increase of ROS. This can well be seen when considering primary mitochondrial diseases, where the tissues with the highest content of mitochondria, namely muscle tissues, are often affected. The result is a variety of movement disorders, including ataxia, dystonia, or tremor [93].

From this perspective, it is interesting to answer the question, how a lack of mitochondrial fidelity can affect other organ systems, preferentially the central nervous system. To follow this question, we analyzed mice that lack the protein PARKIN, which plays an important role in mitophagy, together with PINK1. In agreement with our study defining important roles of PINK1 in the cytosol, the respective mouse mutants for PINK1 or PARKIN show dopaminergic deficits at higher ages $[34,36]$, altered $\mathrm{Ca}^{2+}$ homeostasis dependent excitability [41, 94], weaker synaptic strength, and altered long-term potentiation (LTP) [95], as well as neuroinflammation [1, 56, 62]. The PARKIN-deficient mouse model, which had been generated before, has a loss-offunction mutation in the Prkn gene (the most common genetic cause found among patients with autosomal recessive early-onset parkinsonism) [36]. These mice show motor and cognitive deficits, a decrease of dopamine transporter proteins, but similar to Pink $1^{-1-}$ mice they show no neurodegeneration or Lewy bodies. In total, this mouse model seems to be a valuable tool to further examine the underlying mechanisms leading to the signs of PD patients.

To get insights into the molecular alterations we attempted to identify the ubiquitylation substrates of the E3-ubiquitin-ligase PARKIN. Previously, several direct PARKIN substrates could be identified by targeted approaches, such as the application of the uncoupling agents CCCP or FCCP and subsequent mass spectrometry and detection of ubiquitylated peptides [96]. Other studies in vitro defined several substrates [97-99], which could not be confirmed in mouse and patient brains [40]. Here, a first hypothesisfree method was applied, by the usage of an antibody against a di-glycine remnant that remains after trypsinization at every ubiquitylation target site with subsequent immune- 
precipitation and identification by mass spectrometry. This was done in aged Prkn ${ }^{-1-}$ mice at the age of 18 months to approximate the situation in human patients as closely as possible. To best depict the situation in PD patients, the focus was on neuronspecific factors, which play a role in axon excitability.

Our results confirmed that the strongest downregulation in ubiquitylation is one transmembrane factor, named PORIN3 (VDAC3). VDAC1-3 had been identified before to be targets of PARKIN [100]. These voltage-gated ion channels are located in the outer mitochondrial membrane and they are responsible for the transport of essential metabolites such as ATP, ADP, pyruvate, malate and they play important roles in calcium homeostasis [101]. Strikingly, the two other highest downregulations in ubiquitylation were found in factors that are also involved in calcium homeostasis. These were the neuron-specific calcium sensor hippocalcin (HPCA) and a calciumbuffering factor named calmodulin (CALM1). HPCA is involved in the activation of KCNQ-dependent potassium currents, which are involved in the regulation of slow after-hyperpolarization (sAHP) after several action potentials and thus, it plays an important role in neurotransmission [102, 103]. Following this finding, we also saw other factors with dysregulated ubiquitylation that play a role in the regulation of the sAHP. These included HCN2 and CACNA1B. Additionally, the G-Protein Subunit Alpha 11 (GNA11), which is involved in the regulation of action potentials in response to extracellular calcium concentrations [104], showed decreased ubiquitylation. This confirms the role of PARKIN for mitochondrial GTPases and made us also focus on G-protein subunits. Validations of the findings at transcript and protein levels with RT-qPCR and quantitative immunoblots were conducted in brain tissues of Parkin-KO mice. The results showed altered transcriptional expression for the calcium pump Atp2a1 and Gna11, while Hpca and Calm1 stayed unchanged. At protein levels, ATP1A2, HPCA, and GNA11 showed increased abundance. Consequently, since Gna11 was altered at transcriptional level its changed protein abundance probably reflected compensational efforts. ATP1A2 and HPCA, instead, might represent novel PARKIN targets.

The molecular findings were then analyzed further by electrophysiological experiments in the locus coeruleus (LC) of acute brain slices from Parkin-KO mice. The locus coeruleus is a small brainstem nucleus that has noradrenergic projections to the whole brain and the loss of LC neurons is a feature of neurodegenerative diseases, such as 
AD and PD $[105,106]$. Our patch-clamp analyses revealed accelerated spontaneous pacemaker frequencies at the age of 12 months, with the afterhyperpolarization amplitude being significantly lowered. In addition, the durations of action potentials were significantly reduced, already at the age of 6 months, while we could not observe any changes in potassium flux and no evidence of changes in KCNQ-channel functionality. The increased neuronal excitability and altered afterhyperpolarization could be specific for PD, since similar observations had been made in other mouse models, such as Pink $1^{-/-}$and SNCA overexpression mice [94, 107].

Previous research on the substrates of PARKIN was conducted in HeLa cells, overexpressing PARKIN [35]. One further approach used human embryonic stem cellderived (hESC) induced neurons (iNeurons) and dopaminergic (DA) neurons [108]. With this technique, the authors could show the reproducibility of HeLa data for other cell types by also identifying for example VDAC as the most abundant substrate. Going further, one study does not only analyze the overexpressing PARKIN, but also takes endogenous protein in iNeurons as a basis for their research [109]. They show the limitations of exogenous PARKIN, since several results are overlapping, but some cannot be reproduced with endogenous protein. In conclusion, the data still also depict a limited view because they always represent only a snapshot of the different cell types and cannot fully model the conditions in the brain.

Thus, our study is the first attempt to depict and analyze the circumstances found in mammalian brains with an unbiased hypothesis-free approach, showing all ubiquitylated factors. Of course, both approaches - total brain analyses of mice and induced pluripotent stem cells (iPSC) - have their advantages and disadvantages. Both are time and resource-consuming, in one case due to the required breeding of several mouse generations and their aging, in the other case due to the costly and time-intensive differentiation of neuronal cells in sufficient quantity. iPSC can be a useful tool, but bear the disadvantage that they lose developmental, environmental, and age-related alterations in epigenetics [110]. Their generation is very slow and shows low efficiency. Thus, the direct conversion of fibroblasts into neurons, which is rapid and has higher reprogramming efficiency holds great promise and can also represent patient features. In contrast, mice represent a more compound picture of the disease, but genetically modified mouse strains demand space, time, bureaucratic efforts, knowledge, and care. Once generated they represent a useful tool for the 
analyses of diseases and allow the analyses of primary cells, whole organs, as well as derived cell lines, such as MEF. In conclusion, both approaches seem to hold great promises and should be used in parallel to identify the limitations of each starting tissue and to validate the findings in the other model, respectively.

Since PD affects so many people worldwide, research in the field is of great importance and could help large populations. This does not only include PD and other ageassociated disease treatments but research around this topic has many more facets. Scientists around the world are trying to reduce the number of animal experiments and especially mouse numbers. Thus, studies that are based on either peripheral cells, body fluid biomarkers, or iPSC are highly welcomed. However, to get insights into what to look for in detail, animal experiments can help. Mice are important in PD research. They usually have a 3-year lifespan and can be aged to get more information on these age-associated diseases, such as PD and AD. However, mice that lack PINK1 or PARKIN show no substantial PD phenotypes [95, 111]. As a consequence, the application of environmental stressors gets more and more important to enhance findings and to transfer the murine brain results to peripheral cells and other models. As explained before, PD as an age-related disease is difficult to treat. For one, the cells of an elderly person have a different metabolic state than those of a young person [112]. These changes include different subcellular systems, such as protein quality control, mitochondria, epigenetics, nutrient-sensing pathways, as well as the interplay between these systems. The differentiation between merely age-caused changes and pathogenic findings is not always obvious. In addition, since there is almost no neurogenesis in the brain, the focus should not only be on treatment but on the general understanding of the underlying pathogenic mechanisms. Thus, the research of PD should be focusing on several different aspects. For one, molecular findings of chronically stressed cells, by for example dysfunctional mitochondria, can give important information on how to prevent the development of the disease and how to prolong the pathogenesis. The importance of mitochondria for cellular fidelity has been known for long. Since the connection between dysfunctional mitochondria and PD has been obvious for several decades now, consequently, healthy mitochondria can also help to prevent the development of PD. Apart from obvious mitochondrial damage through for example drugs or pollutants [113], we have now shown that nutritional changes can also have an impact on various cellular pathways. The impact of iron 
levels on the health of cells cannot be denied. This was also studied in humans in the Cypriot population [114]. The results show that manifold environmental factors influence the development of PD. These included pesticides and other toxic substances, nuts consumption, red meat consumption, and soft drinks consumption. The latter one can have effects by the intake of metals that stem from the cans in which the drinks are sold and served. These results from a small population, that otherwise is reported to be among the healthiest and long-living, shows the importance to pursue the impact of such easy-to-apply measures [115].

After prevention, the next findings from research can help to precociously identify disease cases and to track disease progression. At present, there is no early biomarker for PD [116]. Recently, a combination of multiple cerebrospinal fluid (CSF) biomarkers has emerged as an accurate diagnostic and prognostic model. This includes for example the levels of $\alpha$-synuclein [116]. However, sampling is a very invasive and also risky method, so regular sample collection is difficult to implement. Thus, drug development and patient care/assessment could both profit from advances in PD research. Our studies open the discussion and pathways to pursue the identification of PD biomarkers in various ways. One would be to use electrophysiological measures in iPSC from patients to identify early changes. The sampling of fibroblasts is also an invasive technique but getting a skin biopsy is less harmful for the human body than introducing a syringe into the spinal canal. Doing patch-clamp in iPSC was already done [117, 118] and could be repeatedly applied to assess PD development. Our results of the inflammation and iron manuscripts also open new paths in biomarker research. Since dysfunctional mitochondria have to be massively damaged to result in acute apoptosis, a rather supple restriction, such as Pink1 or Prkn mutations does not become immediately obvious. Our data now hint towards the application of stressors to increase cellular reactions that show abnormal levels. The readout of such experiments, for example with the application of an inflammatory stressor like Poly(I:C) would be very different from dopaminergic markers. They could be of inflammatory nature with a focus on pro-inflammatory repressors of toxic RNA/DNA, ubiquitination, and stress response factors. Of course, as shown in our manuscript, there will not be one single factor to focus on, but the combination of various biomarkers could provide a characteristic signature that may reach specificity for PD. 
Coming to treatment, our iron study shows that every therapy will have to be handled with care and can have various side effects. Thus, knowing the affected pathways also after the employment of seemingly harmless measures, such as dietary restrictions for example through iron chelators - is of great importance. This holds not only true for the treatment of PD but also for the prevention of negative side effects, such as missing nucleotide surveillance and the generation of imbalanced mutation rates. Overall, there still is a long way to go from getting further insights into the development and pathophysiology of the monogenic PD variants to then transmitting these to idiopathic cases that are much higher in numbers.

In the long term, the focus will then have to go also away from only dopaminergic cells and brain tissues and move towards the application of peripheral cells with the potential application of external stressors. 


\section{Conclusion}

PD has been known for a very long time and was even mentioned in ancient literature [119], such as Indian and Egyptian texts and in the bible. The first medical description was made in 1817 in James Parkinson's Essay on the Shaking Palsy [120]. Research has progressed fast in the last decades. The first gene to be involved in the development of PD, SNCA, was discovered only in 1997 [26] and up to date, there are 18 PARK-designated PD-related loci [25]. Since monogenic PD cases represent only $5 \%$ of the sporadic cases, the long-term goal should be to also understand the rest $95 \%$ of disease pathogeneses. However, the starting point is through the known genetic loci.

Thus, our studies now contribute to the generation of more knowledge on PD and especially to the connection between mitochondrial dysfunction and the alterations in the cellular context. The approaches of the three studies are highly comprehensive and through the applications of hypotheses-free OMICs allow a very broad increase in knowledge. For the first time, we now generated the global transcriptome of Pink1mutant mouse cerebelli in three different ages, the proteomes of Pink1-depleted MEF in the presence of different extracellular iron concentrations, and the global ubiquitylome of Prkn-knockout mouse brains. The relevant findings were then validated by various techniques and in different species to get ideas on the transferability into human diseases.

Altogether, the three studies show that if mitochondrial dysfunction persists chronically it can have wide-ranging effects on the health of the nervous system. During the long lifespan of a human, also small abnormalities, such as mutations or environmental factors like iron can make the difference over health or disease! 


\section{List of Figures}

Figure 1: Occasional situations of thesis production. ....................................... ii

Figure 2: Scheme of PINK1/PARKIN regulated mitophagy..............................17

Figure 3: Iron metabolism in an exemplary cell. ......................................... 19

Figure 4: Scheme of PINK1-signaling in innate immunity...............................24

Figure 5: Summary of the relevant findings of iron levels in dependency on PINK1. 30 


\section{List of Abbreviations}

22BP

ABCE1

ACO1

AD

ATP

ATP1A2

ATP2A1

BECN1

C. elegans

$\mathrm{Ca}$

CACNA1B

CALM1

CCCP

cGAS

CLPP

CODAS

CPT1A

CREBBP

CREG1

$\mathrm{CSF}$

D. melanogaster

DA

DAMPs

DDX58

DFO

DNA

dsRNA

ER

ES

ETC

FA

FAC
2,2'-Bipyridyl

ATP binding cassette subfamily $E$ member 1

Aconitase 1

Alzheimer's disease

adenosine triphosphate

ATPase $\mathrm{Na}+/ \mathrm{K}+$ Transporting Subunit Alpha 2

ATPase sarcoplasmic/endoplasmic reticulum $\mathrm{Ca}^{2+}$ transporting 1 beclin 1

Caenorhabditis elegans

Calcium

calcium voltage-gated channel subunit alpha1 B

calmodulin 1

carbonylcyanide-3-chlorophenylhydrazone

cyclic GMP-AMP synthase

caseinolytic mitochondrial matrix peptidase proteolytic subunit

Cerebral, ocular, dental, auricular, and skeletal syndrome

carnitine palmitoyltransferase $1 \mathrm{~A}$

CREB Binding Protein

cellular repressor of E1A stimulated genes 1

cerebrospinal fluid

Drosophila melanogaster

dopaminergic

damage-associated molecular patterns

DExD/H-Box Helicase 58

deferoxamine

deoxyribonucleic acid

double-stranded RNA

endoplasmic reticulum

embryonic stem

electron transfer chain

Friedreich's ataxia

ferric ammonium citrate 


\begin{tabular}{|c|c|}
\hline FCCP & carbonylcyanide-4-trifluoromethoxyphenylhydrazone \\
\hline $\mathrm{Fe}$ & iron \\
\hline $\mathrm{FECH}$ & ferrochelatase \\
\hline FTH1 & ferritin heavy chain 1 \\
\hline FTL1 & ferritin light chain 1 \\
\hline GLRX5 & glutaredoxin 5 \\
\hline GNA11 & G protein subunit alpha 11 \\
\hline GSEA & gene set enrichment analysis \\
\hline $\mathrm{h}$ & hour \\
\hline $\mathrm{HCN} 2$ & $\begin{array}{l}\text { hyperpolarization activated cyclic nucleotide gated potassium and } \\
\text { sodium channel } 2\end{array}$ \\
\hline hESC & human embryonic stem cell \\
\hline HMOX1 & heme oxygenase 1 \\
\hline HPCA & hippocalcin \\
\hline IFIT1/2/3 & interferon induced protein with tetratricopeptide repeats $1 / 3$ \\
\hline IKK & inhibitor of nuclear factor kappa B \\
\hline IMPC & international mouse phenotyping consortium \\
\hline iPSC & induced pluripotent stem cell \\
\hline IRF3/7 & interferon response factor $3 / 7$ \\
\hline IRP1/2 & iron-responsive element-binding protein 1/2 \\
\hline ISC & iron-sulfur-cluster \\
\hline IKB & I-Kappa-B \\
\hline $\mathrm{KCNQ}$ & potassium voltage-gated channel subfamily $Q$ \\
\hline $\mathrm{KO}$ & knockout \\
\hline LC & locus coeruleus \\
\hline LIP & labile iron pool \\
\hline LIPA & lipase A \\
\hline LONP1 & Lon peptidase 1, Mitochondrial \\
\hline LTP & long-term potentiation \\
\hline MAM & mitochondria-associated membranes \\
\hline MAP1LC3A & microtubule associated protein 1 light chain 3 alpha \\
\hline MAPK & mitogen-activated protein kinase \\
\hline MAVS & mitochondrial antiviral signalling protein \\
\hline MDA5 & melanoma differentiation-associated gene 5 \\
\hline MEF & mouse embryonic fibroblast \\
\hline
\end{tabular}


MELAS

MFN1

MFRN

MMP14

MPP $^{+}$

MPTP

MRPS35/36

mtDNA

MTS

MYD88

NADPH

NFKB

NLRP3

NQO1

NRF2

NTHL1

OMM

OTC

OXPHOS

PARL

PD

PINK1

PMD

Poly(I:C)

PRKN

PYGL

RIG-I

RNA

ROS

RRM1/2

RSAD2

RT-qPCR

sAHP

SMD mitochondrial encephalopathy, lactic acidosis, and stroke-like episodes

Mitofusin 1

mitoferritin

matrix metallopeptidase 14

1-methyl-4-phenylpyridinium

1-methyl-4-phenyl-1,2,3,6-tetrahydropyridine

mitochondrial ribosomal protein S35/36

mitochondrial DNA

mitochondrial target sequence

myeloid differentiation primary response protein MyD88

nicotinamide adenine dinucleotide phosphate

nuclear factor kappa B

NLR Family Pyrin Domain Containing 3

$\mathrm{NAD}(\mathrm{P}) \mathrm{H}$ quinone dehydrogenase 1

nuclear factor, erythroid 2 like 2

Nth like DNA glycosylase 1

outer mitochondrial membrane

organotypic slice cultures

oxidative phosphorylation

Presenilins-associated rhomboid-like protein

Parkinson's disease

PTEN induced kinase 1

primary mitochondrial disease

Polyinosinic:polycytidylic acid

PARKIN

glycogen phosphorylase L

antiviral innate immune response receptor RIG-I

ribonucleic acid

reactive oxygen species

ribonucleotide reductase catalytic subunit M1/M2

radical S-adenosyl methionine domain containing 2

reverse transcriptase quantitative polymerase chain reaction

slow after-hyperpolarization

secondary mitochondrial disease 


$\begin{array}{ll}\text { SNCA } & \text { alpha-synuclein } \\ \text { SNCB } & \text { beta-synuclein } \\ \text { SNpc } & \text { substantia nigra pars compacta } \\ \text { SRSF10 } & \text { serine and arginine rich splicing factor } 10 \\ \text { SSRNA } & \text { single-stranded RNA } \\ \text { STING } & \text { signaling protein stimulator of interferon genes } \\ \text { STRING } & \text { search tool for recurring instances of neighboring genes } \\ \text { TCA } & \text { tricarboxylic acid cycle } \\ \text { TFR1 } & \text { transferrin receptor } 1 \\ \text { TLR9 } & \text { Toll-like receptor } 9 \\ \text { TRADD } & \text { tumor necrosis factor receptor type 1 associated via death domain } \\ \text { TRAF } & \text { TNF receptor associated factor } \\ \text { UPRmt } & \text { unfolded protein response, mitochondrial } \\ \text { VDAC1/3 } & \text { voltage dependent anion channel } 1 / 3 \\ \text { WT } & \text { wildtype } \\ \text { XDH } & \text { xanthine dehydrogenase }\end{array}$




\section{References}

1. Torres-Odio, S.; Key, J.; Hoepken, H. H.; Canet-Pons, J.; Valek, L.; Roller, B.; Walter, M.; Morales-Gordo, B.; Meierhofer, D.; Harter, P. N.; Mittelbronn, M.; Tegeder, I.; Gispert, S.; Auburger, G., Progression of pathology in PINK1-deficient mouse brain from splicing via ubiquitination, ER stress, and mitophagy changes to neuroinflammation. J Neuroinflammation 2017, 14, (1), 154.

2. Gray, M. W., The endosymbiont hypothesis revisited. Int Rev Cytol 1992, 141, 233357.

3. Kandel, E. R.; Mack, S.; Jessell, T. M.; Schwartz, J. H.; Siegelbaum, S. A.; Hudspeth, A. J., Principles of Neural Science, Fifth Edition. McGraw-Hill Education: 2013.

4. Lane, N.; Martin, W., The energetics of genome complexity. Nature 2010, 467, (7318), 929-34.

5. West, A. P.; Shadel, G. S.; Ghosh, S., Mitochondria in innate immune responses. Nat Rev Immunol 2011, 11, (6), 389-402.

6. Rabiet, M. J.; Huet, E.; Boulay, F., Human mitochondria-derived N-formylated peptides are novel agonists equally active on FPR and FPRL1, while Listeria monocytogenesderived peptides preferentially activate FPR. Eur J Immunol 2005, 35, (8), 2486-95.

7. Green, D. R.; Van Houten, B., SnapShot: Mitochondrial quality control. Cell 2011, 147, (4), 950, 950 e1.

8. Fiorese, C. J.; Haynes, C. M., Integrating the $\operatorname{UPR}(\mathrm{mt})$ into the mitochondrial maintenance network. Crit Rev Biochem Mol Biol 2017, 52, (3), 304-313.

9. Duchen, M. R., Mitochondria and calcium: from cell signalling to cell death. J Physiol 2000, 529 Pt 1, 57-68.

10. Mills, E. L.; Kelly, B.; O'Neill, L. A. J., Mitochondria are the powerhouses of immunity. Nat Immunol 2017, 18, (5), 488-498.

11. West, A. P., Mitochondrial dysfunction as a trigger of innate immune responses and inflammation. Toxicology 2017, 391, 54-63.

12. Bock, F. J.; Tait, S. W. G., Mitochondria as multifaceted regulators of cell death. Nat Rev Mol Cell Biol 2020, 21, (2), 85-100.

13. Lake, N. J.; Bird, M. J.; Isohanni, P.; Paetau, A., Leigh syndrome: neuropathology and pathogenesis. J Neuropathol Exp Neurol 2015, 74, (6), 482-92.

14. El-Hattab, A. W.; Adesina, A. M.; Jones, J.; Scaglia, F., MELAS syndrome: Clinical manifestations, pathogenesis, and treatment options. Mol Genet Metab 2015, 116, (12), 4-12.

15. Yoon, Y.; Galloway, C. A.; Jhun, B. S.; Yu, T., Mitochondrial dynamics in diabetes. Antioxid Redox Signal 2011, 14, (3), 439-57.

16. Konradi, C.; Ongur, D., Role of mitochondria and energy metabolism in schizophrenia and psychotic disorders. Schizophr Res 2017, 187, 1-2.

17. Boland, M. L.; Chourasia, A. H.; Macleod, K. F., Mitochondrial dysfunction in cancer. Front Oncol 2013, 3, 292.

18. Koeppen, A. H., Friedreich's ataxia: pathology, pathogenesis, and molecular genetics. J Neurol Sci 2011, 303, (1-2), 1-12.

19. Lin, M. T.; Beal, M. F., Mitochondrial dysfunction and oxidative stress in neurodegenerative diseases. Nature 2006, 443, (7113), 787-95.

20. Lebouvier, T.; Chaumette, T.; Paillusson, S.; Duyckaerts, C.; Bruley des Varannes, S.; Neunlist, M.; Derkinderen, P., The second brain and Parkinson's disease. Eur $J$ Neurosci 2009, 30, (5), 735-41.

21. de Rijk, M. C.; Breteler, M. M.; Graveland, G. A.; Ott, A.; Grobbee, D. E.; van der Meche, F. G.; Hofman, A., Prevalence of Parkinson's disease in the elderly: the Rotterdam Study. Neurology 1995, 45, (12), 2143-6. 
22. Modugno, N.; Lena, F.; Di Biasio, F.; Cerrone, G.; Ruggieri, S.; Fornai, F., A clinical overview of non-motor symptoms in Parkinson's Disease. Arch Ital Biol 2013, 151, (4), 148-68.

23. Braak, H.; Del Tredici, K.; Rub, U.; de Vos, R. A.; Jansen Steur, E. N.; Braak, E., Staging of brain pathology related to sporadic Parkinson's disease. Neurobiol Aging 2003, 24, (2), 197-211.

24. Prakash, K. G.; Bannur, B. M.; Chavan, M. D.; Saniya, K.; Sailesh, K. S.; Rajagopalan, A., Neuroanatomical changes in Parkinson's disease in relation to cognition: An update. J Adv Pharm Technol Res 2016, 7, (4), 123-126.

25. Klein, C.; Westenberger, A., Genetics of Parkinson's disease. Cold Spring Harb Perspect Med 2012, 2, (1), a008888.

26. Polymeropoulos, M. H.; Lavedan, C.; Leroy, E.; Ide, S. E.; Dehejia, A.; Dutra, A.; Pike, B.; Root, H.; Rubenstein, J.; Boyer, R.; Stenroos, E. S.; Chandrasekharappa, S.; Athanassiadou, A.; Papapetropoulos, T.; Johnson, W. G.; Lazzarini, A. M.; Duvoisin, R. C.; Di lorio, G.; Golbe, L. I.; Nussbaum, R. L., Mutation in the alpha-synuclein gene identified in families with Parkinson's disease. Science 1997, 276, (5321), 2045-7.

27. Valente, E. M.; Abou-Sleiman, P. M.; Caputo, V.; Muqit, M. M.; Harvey, K.; Gispert, S.; Ali, Z.; Del Turco, D.; Bentivoglio, A. R.; Healy, D. G.; Albanese, A.; Nussbaum, R.; Gonzalez-Maldonado, R.; Deller, T.; Salvi, S.; Cortelli, P.; Gilks, W. P.; Latchman, D. S.; Harvey, R. J.; Dallapiccola, B.; Auburger, G.; Wood, N. W., Hereditary early-onset Parkinson's disease caused by mutations in PINK1. Science 2004, 304, (5674), 115860.

28. Kitada, T.; Asakawa, S.; Hattori, N.; Matsumine, H.; Yamamura, Y.; Minoshima, S.; Yokochi, M.; Mizuno, Y.; Shimizu, N., Mutations in the parkin gene cause autosomal recessive juvenile parkinsonism. Nature 1998, 392, (6676), 605-8.

29. Zhang, C. W.; Hang, L.; Yao, T. P.; Lim, K. L., Parkin Regulation and Neurodegenerative Disorders. Front Aging Neurosci 2015, 7, 248.

30. Zhou, C.; Huang, Y.; Shao, Y.; May, J.; Prou, D.; Perier, C.; Dauer, W.; Schon, E. A.; Przedborski, S., The kinase domain of mitochondrial PINK1 faces the cytoplasm. Proc Natl Acad Sci U S A 2008, 105, (33), 12022-7.

31. Lin, W.; Kang, U. J., Characterization of PINK1 processing, stability, and subcellular localization. J Neurochem 2008, 106, (1), 464-74.

32. Pickrell, A. M.; Youle, R. J., The roles of PINK1, parkin, and mitochondrial fidelity in Parkinson's disease. Neuron 2015, 85, (2), 257-73.

33. Klinkenberg, M.; Gispert, S.; Dominguez-Bautista, J. A.; Braun, I.; Auburger, G.; Jendrach, M., Restriction of trophic factors and nutrients induces PARKIN expression. Neurogenetics 2012, 13, (1), 9-21.

34. Gispert, S.; Ricciardi, F.; Kurz, A.; Azizov, M.; Hoepken, H. H.; Becker, D.; Voos, W.; Leuner, K.; Muller, W. E.; Kudin, A. P.; Kunz, W. S.; Zimmermann, A.; Roeper, J.; Wenzel, D.; Jendrach, M.; Garcia-Arencibia, M.; Fernandez-Ruiz, J.; Huber, L.; Rohrer, H.; Barrera, M.; Reichert, A. S.; Rub, U.; Chen, A.; Nussbaum, R. L.; Auburger, G., Parkinson phenotype in aged PINK1-deficient mice is accompanied by progressive mitochondrial dysfunction in absence of neurodegeneration. PLoS One 2009, 4, (6), e5777.

35. Narendra, D.; Tanaka, A.; Suen, D. F.; Youle, R. J., Parkin is recruited selectively to impaired mitochondria and promotes their autophagy. J Cell Biol 2008, 183, (5), 795803.

36. Itier, J. M.; Ibanez, P.; Mena, M. A.; Abbas, N.; Cohen-Salmon, C.; Bohme, G. A.; Laville, M.; Pratt, J.; Corti, O.; Pradier, L.; Ret, G.; Joubert, C.; Periquet, M.; Araujo, F.; Negroni, J.; Casarejos, M. J.; Canals, S.; Solano, R.; Serrano, A.; Gallego, E.; Sanchez, M.; Denefle, P.; Benavides, J.; Tremp, G.; Rooney, T. A.; Brice, A.; Garcia de Yebenes, J., Parkin gene inactivation alters behaviour and dopamine neurotransmission in the mouse. Hum Mol Genet 2003, 12, (18), 2277-91. 
37. Hanson, J. E.; Orr, A. L.; Madison, D. V., Altered hippocampal synaptic physiology in aged parkin-deficient mice. Neuromolecular Med 2010, 12, (3), 270-6.

38. Noda, S.; Sato, S.; Fukuda, T.; Tada, N.; Uchiyama, Y.; Tanaka, K.; Hattori, N., Loss of Parkin contributes to mitochondrial turnover and dopaminergic neuronal loss in aged mice. Neurobiol Dis 2020, 136, 104717.

39. Perez, F. A.; Palmiter, R. D., Parkin-deficient mice are not a robust model of parkinsonism. Proc Natl Acad Sci U S A 2005, 102, (6), 2174-9.

40. Periquet, M.; Corti, O.; Jacquier, S.; Brice, A., Proteomic analysis of parkin knockout mice: alterations in energy metabolism, protein handling and synaptic function. $J$ Neurochem 2005, 95, (5), 1259-76.

41. Gautier, C. A.; Erpapazoglou, Z.; Mouton-Liger, F.; Muriel, M. P.; Cormier, F.; Bigou, S.; Duffaure, S.; Girard, M.; Foret, B.; Iannielli, A.; Broccoli, V.; Dalle, C.; Bohl, D.; Michel, P. P.; Corvol, J. C.; Brice, A.; Corti, O., The endoplasmic reticulummitochondria interface is perturbed in PARK2 knockout mice and patients with PARK2 mutations. Hum Mol Genet 2016, 25, (14), 2972-2984.

42. Martin, W. R.; Wieler, M.; Gee, M., Midbrain iron content in early Parkinson disease: a potential biomarker of disease status. Neurology 2008, 70, (16 Pt 2), 1411-7.

43. Eid, R.; Arab, N. T.; Greenwood, M. T., Iron mediated toxicity and programmed cell death: A review and a re-examination of existing paradigms. Biochim Biophys Acta Mol Cell Res 2017, 1864, (2), 399-430.

44. Mena, N. P.; Urrutia, P. J.; Lourido, F.; Carrasco, C. M.; Nunez, M. T., Mitochondrial iron homeostasis and its dysfunctions in neurodegenerative disorders. Mitochondrion 2015, 21, 92-105.

45. Stehling, O.; Lill, R., The role of mitochondria in cellular iron-sulfur protein biogenesis: mechanisms, connected processes, and diseases. Cold Spring Harb Perspect Biol 2013, 5, (8), a011312.

46. Shkreta, L.; Toutant, J.; Durand, M.; Manley, J. L.; Chabot, B., SRSF10 Connects DNA Damage to the Alternative Splicing of Transcripts Encoding Apoptosis, Cell-Cycle Control, and DNA Repair Factors. Cell Rep 2016, 17, (8), 1990-2003.

47. Gispert, S.; Brehm, N.; Weil, J.; Seidel, K.; Rub, U.; Kern, B.; Walter, M.; Roeper, J.; Auburger, G., Potentiation of neurotoxicity in double-mutant mice with Pink1 ablation and A53T-SNCA overexpression. Hum Mol Genet 2015, 24, (4), 1061-76.

48. Auburger, G.; Gispert, S.; Torres-Odio, S.; Jendrach, M.; Brehm, N.; Canet-Pons, J.; Key, J.; Sen, N. E., SerThr-PhosphoProteome of Brain from Aged PINK1-KO+A53TSNCA Mice Reveals pT1928-MAP1B and pS3781-ANK2 Deficits, as Hub between Autophagy and Synapse Changes. Int J Mol Sci 2019, 20, (13).

49. Banoth, B.; Cassel, S. L., Mitochondria in innate immune signaling. Trans/ Res 2018, 202, 52-68.

50. Mathur, V.; Burai, R.; Vest, R. T.; Bonanno, L. N.; Lehallier, B.; Zardeneta, M. E.; Mistry, K. N.; Do, D.; Marsh, S. E.; Abud, E. M.; Blurton-Jones, M.; Li, L.; Lashuel, H. A.; WyssCoray, T., Activation of the STING-Dependent Type I Interferon Response Reduces Microglial Reactivity and Neuroinflammation. Neuron 2017, 96, (6), 1290-1302 e6.

51. Nolan, Y. M.; Sullivan, A. M.; Toulouse, A., Parkinson's disease in the nuclear age of neuroinflammation. Trends Mol Med 2013, 19, (3), 187-96.

52. More, S. V.; Kumar, H.; Kim, I. S.; Song, S. Y.; Choi, D. K., Cellular and molecular mediators of neuroinflammation in the pathogenesis of Parkinson's disease. Mediators Inflamm 2013, 2013, 952375.

53. Mosley, R. L.; Benner, E. J.; Kadiu, I.; Thomas, M.; Boska, M. D.; Hasan, K.; Laurie, C.; Gendelman, H. E., Neuroinflammation, Oxidative Stress and the Pathogenesis of Parkinson's Disease. Clin Neurosci Res 2006, 6, (5), 261-281.

54. Manzanillo, P. S.; Ayres, J. S.; Watson, R. O.; Collins, A. C.; Souza, G.; Rae, C. S.; Schneider, D. S.; Nakamura, K.; Shiloh, M. U.; Cox, J. S., The ubiquitin ligase parkin mediates resistance to intracellular pathogens. Nature 2013, 501, (7468), 512-6. 
55. Matheoud, D.; Sugiura, A.; Bellemare-Pelletier, A.; Laplante, A.; Rondeau, C.; Chemali, M.; Fazel, A.; Bergeron, J. J.; Trudeau, L. E.; Burelle, Y.; Gagnon, E.; McBride, H. M.; Desjardins, M., Parkinson's Disease-Related Proteins PINK1 and Parkin Repress Mitochondrial Antigen Presentation. Cell 2016, 166, (2), 314-327.

56. Mouton-Liger, F.; Rosazza, T.; Sepulveda-Diaz, J.; leang, A.; Hassoun, S. M.; Claire, E.; Mangone, G.; Brice, A.; Michel, P. P.; Corvol, J. C.; Corti, O., Parkin deficiency modulates NLRP3 inflammasome activation by attenuating an A20-dependent negative feedback loop. Glia 2018, 66, (8), 1736-1751.

57. West, A. P.; Shadel, G. S., Mitochondrial DNA in innate immune responses and inflammatory pathology. Nat Rev Immunol 2017, 17, (6), 363-375.

58. Zhang, Q.; Raoof, M.; Chen, Y.; Sumi, Y.; Sursal, T.; Junger, W.; Brohi, K.; Itagaki, K.; Hauser, C. J., Circulating mitochondrial DAMPs cause inflammatory responses to injury. Nature 2010, 464, (7285), 104-7.

59. Motwani, M.; Pesiridis, S.; Fitzgerald, K. A., DNA sensing by the cGAS-STING pathway in health and disease. Nat Rev Genet 2019, 20, (11), 657-674.

60. Gispert, S.; Parganlija, D.; Klinkenberg, M.; Drose, S.; Wittig, I.; Mittelbronn, M.; Grzmil, P.; Koob, S.; Hamann, A.; Walter, M.; Buchel, F.; Adler, T.; Hrabe de Angelis, M.; Busch, D. H.; Zell, A.; Reichert, A. S.; Brandt, U.; Osiewacz, H. D.; Jendrach, M.; Auburger, G., Loss of mitochondrial peptidase Clpp leads to infertility, hearing loss plus growth retardation via accumulation of CLPX, mtDNA and inflammatory factors. Hum Mol Genet 2013, 22, (24), 4871-87.

61. Newman, W. G.; Friedman, T. B.; Conway, G. S.; Demain, L. A. M., Perrault Syndrome. In GeneReviews((R)), Adam, M. P.; Ardinger, H. H.; Pagon, R. A.; Wallace, S. E.; Bean, L. J. H.; Mirzaa, G.; Amemiya, A., Eds. Seattle (WA), 1993.

62. Sliter, D. A.; Martinez, J.; Hao, L.; Chen, X.; Sun, N.; Fischer, T. D.; Burman, J. L.; Li, Y.; Zhang, Z.; Narendra, D. P.; Cai, H.; Borsche, M.; Klein, C.; Youle, R. J., Parkin and PINK1 mitigate STING-induced inflammation. Nature 2018, 561, (7722), 258-262.

63. Chin, A. C., Neuroinflammation and the cGAS-STING pathway. J Neurophysiol 2019, 121, (4), 1087-1091.

64. Bender, A.; Krishnan, K. J.; Morris, C. M.; Taylor, G. A.; Reeve, A. K.; Perry, R. H.; Jaros, E.; Hersheson, J. S.; Betts, J.; Klopstock, T.; Taylor, R. W.; Turnbull, D. M., High levels of mitochondrial DNA deletions in substantia nigra neurons in aging and Parkinson disease. Nat Genet 2006, 38, (5), 515-7.

65. Davis, G. C.; Williams, A. C.; Markey, S. P.; Ebert, M. H.; Caine, E. D.; Reichert, C. M.; Kopin, I. J., Chronic Parkinsonism secondary to intravenous injection of meperidine analogues. Psychiatry Res 1979, 1, (3), 249-54.

66. Nonnekes, J.; Post, B.; Tetrud, J. W.; Langston, J. W.; Bloem, B. R., MPTP-induced parkinsonism: an historical case series. Lancet Neurol 2018, 17, (4), 300-301.

67. Burns, R. S.; Chiueh, C. C.; Markey, S. P.; Ebert, M. H.; Jacobowitz, D. M.; Kopin, I. J., A primate model of parkinsonism: selective destruction of dopaminergic neurons in the pars compacta of the substantia nigra by $\mathrm{N}$-methyl-4-phenyl-1,2,3,6tetrahydropyridine. Proc Natl Acad Sci U S A 1983, 80, (14), 4546-50.

68. Cleeter, M. W.; Cooper, J. M.; Schapira, A. H., Irreversible inhibition of mitochondrial complex I by 1-methyl-4-phenylpyridinium: evidence for free radical involvement. $J$ Neurochem 1992, 58, (2), 786-9.

69. Nicklas, W. J.; Vyas, I.; Heikkila, R. E., Inhibition of NADH-linked oxidation in brain mitochondria by 1-methyl-4-phenyl-pyridine, a metabolite of the neurotoxin, 1-methyl4-phenyl-1,2,5,6-tetrahydropyridine. Life Sci 1985, 36, (26), 2503-8.

70. Porras, G.; Li, Q.; Bezard, E., Modeling Parkinson's disease in primates: The MPTP model. Cold Spring Harb Perspect Med 2012, 2, (3), a009308.

71. Meredith, G. E.; Rademacher, D. J., MPTP mouse models of Parkinson's disease: an update. J Parkinsons Dis 2011, 1, (1), 19-33.

72. Heytler, P. G.; Prichard, W. W., A new class of uncoupling agents--carbonyl cyanide phenylhydrazones. Biochem Biophys Res Commun 1962, 7, 272-5. 
73. Logroscino, G.; Marder, K.; Graziano, J.; Freyer, G.; Slavkovich, V.; Lolacono, N.; Cote, L.; Mayeux, R., Altered systemic iron metabolism in Parkinson's disease. Neurology 1997, 49, (3), 714-7.

74. Uranga, R. M.; Salvador, G. A., Unraveling the Burden of Iron in Neurodegeneration: Intersections with Amyloid Beta Peptide Pathology. Oxid Med Cell Longev 2018, 2018, 2850341.

75. Weiland, A.; Wang, Y.; Wu, W.; Lan, X.; Han, X.; Li, Q.; Wang, J., Ferroptosis and Its Role in Diverse Brain Diseases. Mol Neurobiol 2019, 56, (7), 4880-4893.

76. Nunez, M. T.; Chana-Cuevas, P., New perspectives in iron chelation therapy for the treatment of Parkinson's disease. Neural Regen Res 2019, 14, (11), 1905-1906.

77. Key, J.; Sen, N. E.; Arsovic, A.; Kramer, S.; Hulse, R.; Khan, N. N.; Meierhofer, D.; Gispert, S.; Koepf, G.; Auburger, G., Systematic Surveys of Iron Homeostasis Mechanisms Reveal Ferritin Superfamily and Nucleotide Surveillance Regulation to be Modified by PINK1 Absence. Cells 2020, 9, (10).

78. Schiavi, A.; Maglioni, S.; Palikaras, K.; Shaik, A.; Strappazzon, F.; Brinkmann, V.; Torgovnick, A.; Castelein, N.; De Henau, S.; Braeckman, B. P.; Cecconi, F.; Tavernarakis, N.; Ventura, N., Iron-Starvation-Induced Mitophagy Mediates Lifespan Extension upon Mitochondrial Stress in C. elegans. Curr Biol 2015, 25, (14), 1810-22.

79. Barthelme, D.; Scheele, U.; Dinkelaker, S.; Janoschka, A.; Macmillan, F.; Albers, S. V.; Driessen, A. J.; Stagni, M. S.; Bill, E.; Meyer-Klaucke, W.; Schunemann, V.; Tampe, R., Structural organization of essential iron-sulfur clusters in the evolutionarily highly conserved ATP-binding cassette protein ABCE1. J Biol Chem 2007, 282, (19), 14598607.

80. Wu, Z.; Wang, Y.; Lim, J.; Liu, B.; Li, Y.; Vartak, R.; Stankiewicz, T.; Montgomery, S.; Lu, B., Ubiquitination of ABCE1 by NOT4 in Response to Mitochondrial Damage Links Co-translational Quality Control to PINK1-Directed Mitophagy. Cell Metab 2018, 28, (1), 130-144 e7.

81. Coelho, C. M.; Kolevski, B.; Bunn, C.; Walker, C.; Dahanukar, A.; Leevers, S. J., Growth and cell survival are unevenly impaired in pixie mutant wing discs. Development 2005, 132, (24), 5411-24.

82. Lill, R.; Freibert, S. A., Mechanisms of Mitochondrial Iron-Sulfur Protein Biogenesis. Annu Rev Biochem 2020, 89, 471-499.

83. Murata, H.; Takamatsu, H.; Liu, S.; Kataoka, K.; Huh, N. H.; Sakaguchi, M., NRF2 Regulates PINK1 Expression under Oxidative Stress Conditions. PLoS One 2015, 10, (11), e0142438.

84. Gu, G.; Reyes, P. E.; Golden, G. T.; Woltjer, R. L.; Hulette, C.; Montine, T. J.; Zhang, J., Mitochondrial DNA deletions/rearrangements in parkinson disease and related neurodegenerative disorders. J Neuropathol Exp Neurol 2002, 61, (7), 634-9.

85. Wan, Z.; Xu, J.; Huang, Y.; Zhai, Y.; Ma, Z.; Zhou, B.; Cao, Z., Elevating bioavailable iron levels in mitochondria suppresses the defective phenotypes caused by PINK1 loss-of-function in Drosophila melanogaster. Biochem Biophys Res Commun 2020, 532, (2), 285-291.

86. Council, U. M. R. International Mouse Phenotyping Consortium. https://www.mousephenotype.org/data/genes/MGl:1858213 05.03.2021),

87. Key, J.; Maletzko, A.; Kohli, A.; Gispert, S.; Torres-Odio, S.; Wittig, I.; Heidler, J.; Barcena, C.; Lopez-Otin, C.; Lei, Y.; West, A. P.; Munch, C.; Auburger, G., Loss of mitochondrial ClpP, Lonp1, and Tfam triggers transcriptional induction of Rnf213, a susceptibility factor for moyamoya disease. Neurogenetics 2020, 21, (3), 187-203.

88. Torres-Odio, S.; Lei, Y.; Gispert, S.; Maletzko, A.; Key, J.; Menissy, S. S.; Wittig, I.; Auburger, G.; West, A. P., Loss of Mitochondrial Protease CLPP Activates Type I IFN Responses through the Mitochondrial DNA-cGAS-STING Signaling Axis. The Journal of Immunology 2021, ji2001016.

89. Key, J.; Kohli, A.; Barcena, C.; Lopez-Otin, C.; Heidler, J.; Wittig, I.; Auburger, G., Global Proteome of LonP1(+/-) Mouse Embryonal Fibroblasts Reveals Impact on 
Respiratory Chain, but No Interdependence between Eral1 and Mitoribosomes. Int $J$ Mol Sci 2019, 20, (18).

90. Key, J.; Harter, P. N.; Sen, N. E.; Gradhand, E.; Auburger, G.; Gispert, S., MidGestation lethality of Atxn2l-Ablated Mice. Int J Mol Sci 2020, 21, (14).

91. Picca, A.; Saini, S. K.; Mankowski, R. T.; Kamenov, G.; Anton, S. D.; Manini, T. M.; Buford, T. W.; Wohlgemuth, S. E.; Xiao, R.; Calvani, R.; Coelho-Junior, H. J.; Landi, F.; Bernabei, R.; Hood, D. A.; Marzetti, E.; Leeuwenburgh, C., Altered Expression of Mitoferrin and Frataxin, Larger Labile Iron Pool and Greater Mitochondrial DNA Damage in the Skeletal Muscle of Older Adults. Cells 2020, 9, (12).

92. Bus, C.; Zizmare, L.; Feldkaemper, M.; Geisler, S.; Zarani, M.; Schaedler, A.; Klose, F.; Admard, J.; Mageean, C. J.; Arena, G.; Fallier-Becker, P.; Ugun-Klusek, A.; Maruszczak, K. K.; Kapolou, K.; Schmid, B.; Rapaport, D.; Ueffing, M.; Casadei, N.; Kruger, R.; Gasser, T.; Vogt Weisenhorn, D. M.; Kahle, P. J.; Trautwein, C.; Gloeckner, C. J.; Fitzgerald, J. C., Human Dopaminergic Neurons Lacking PINK1 Exhibit Disrupted Dopamine Metabolism Related to Vitamin B6 Co-Factors. iScience 2020, 23, (12), 101797.

93. Ghaoui, R.; Sue, C. M., Movement disorders in mitochondrial disease. J Neurol 2018, 265, (5), 1230-1240.

94. Bishop, M. W.; Chakraborty, S.; Matthews, G. A.; Dougalis, A.; Wood, N. W.; Festenstein, R.; Ungless, M. A., Hyperexcitable substantia nigra dopamine neurons in PINK1- and HtrA2/Omi-deficient mice. J Neurophysiol 2010, 104, (6), 3009-20.

95. Kitada, T.; Pisani, A.; Porter, D. R.; Yamaguchi, H.; Tscherter, A.; Martella, G.; Bonsi, P.; Zhang, C.; Pothos, E. N.; Shen, J., Impaired dopamine release and synaptic plasticity in the striatum of PINK1-deficient mice. Proc Natl Acad Sci U S A 2007, 104, (27), 11441-6.

96. Ordureau, A.; Heo, J. M.; Duda, D. M.; Paulo, J. A.; Olszewski, J. L.; Yanishevski, D.; Rinehart, J.; Schulman, B. A.; Harper, J. W., Defining roles of PARKIN and ubiquitin phosphorylation by PINK1 in mitochondrial quality control using a ubiquitin replacement strategy. Proc Natl Acad Sci U S A 2015, 112, (21), 6637-42.

97. Imai, Y.; Takahashi, R., How do Parkin mutations result in neurodegeneration? Curr Opin Neurobiol 2004, 14, (3), 384-9.

98. Savitt, J. M.; Dawson, V. L.; Dawson, T. M., Diagnosis and treatment of Parkinson disease: molecules to medicine. J Clin Invest 2006, 116, (7), 1744-54.

99. Chan, N. C.; Salazar, A. M.; Pham, A. H.; Sweredoski, M. J.; Kolawa, N. J.; Graham, R. L.; Hess, S.; Chan, D. C., Broad activation of the ubiquitin-proteasome system by Parkin is critical for mitophagy. Hum Mol Genet 2011, 20, (9), 1726-37.

100. Geisler, S.; Holmstrom, K. M.; Skujat, D.; Fiesel, F. C.; Rothfuss, O. C.; Kahle, P. J.; Springer, W., PINK1/Parkin-mediated mitophagy is dependent on VDAC1 and p62/SQSTM1. Nat Cell Biol 2010, 12, (2), 119-31.

101. Blachly-Dyson, E.; Forte, M., VDAC channels. IUBMB Life 2001, 52, (3-5), 113-8.

102. Kim, K. S.; Kobayashi, M.; Takamatsu, K.; Tzingounis, A. V., Hippocalcin and KCNQ channels contribute to the kinetics of the slow afterhyperpolarization. Biophys $\mathrm{J} 2012$, 103, (12), 2446-54.

103. Tzingounis, A. V.; Kobayashi, M.; Takamatsu, K.; Nicoll, R. A., Hippocalcin gates the calcium activation of the slow afterhyperpolarization in hippocampal pyramidal cells. Neuron 2007, 53, (4), 487-93.

104. Pahlavan, S.; Oberhofer, M.; Sauer, B.; Ruppenthal, S.; Tian, Q.; Scholz, A.; Kaestner, L.; Lipp, P., Galphaq and Galpha11 contribute to the maintenance of cellular electrophysiology and $\mathrm{Ca} 2+$ handling in ventricular cardiomyocytes. Cardiovasc Res 2012, 95, (1), 48-58.

105. Janitzky, K., Impaired Phasic Discharge of Locus Coeruleus Neurons Based on Persistent High Tonic Discharge-A New Hypothesis With Potential Implications for Neurodegenerative Diseases. Front Neurol 2020, 11, 371. 
106. Von Coelln, R.; Thomas, B.; Savitt, J. M.; Lim, K. L.; Sasaki, M.; Hess, E. J.; Dawson, V. L.; Dawson, T. M., Loss of locus coeruleus neurons and reduced startle in parkin null mice. Proc Natl Acad Sci U S A 2004, 101, (29), 10744-9.

107. Subramaniam, M.; Althof, D.; Gispert, S.; Schwenk, J.; Auburger, G.; Kulik, A.; Fakler, B.; Roeper, J., Mutant alpha-synuclein enhances firing frequencies in dopamine substantia nigra neurons by oxidative impairment of A-type potassium channels. $J$ Neurosci 2014, 34, (41), 13586-99.

108. Ordureau, A.; Paulo, J. A.; Zhang, W.; Ahfeldt, T.; Zhang, J.; Cohn, E. F.; Hou, Z.; Heo, J. M.; Rubin, L. L.; Sidhu, S. S.; Gygi, S. P.; Harper, J. W., Dynamics of PARKINDependent Mitochondrial Ubiquitylation in Induced Neurons and Model Systems Revealed by Digital Snapshot Proteomics. Mol Cell 2018, 70, (2), 211-227 e8.

109. Ordureau, A.; Paulo, J. A.; Zhang, J.; An, H.; Swatek, K. N.; Cannon, J. R.; Wan, Q.; Komander, D.; Harper, J. W., Global Landscape and Dynamics of Parkin and USP30Dependent Ubiquitylomes in iNeurons during Mitophagic Signaling. Mol Cell 2020, 77, (5), 1124-1142 e10.

110. Kyttala, A.; Moraghebi, R.; Valensisi, C.; Kettunen, J.; Andrus, C.; Pasumarthy, K. K.; Nakanishi, M.; Nishimura, K.; Ohtaka, M.; Weltner, J.; Van Handel, B.; Parkkonen, O.; Sinisalo, J.; Jalanko, A.; Hawkins, R. D.; Woods, N. B.; Otonkoski, T.; Trokovic, R., Genetic Variability Overrides the Impact of Parental Cell Type and Determines iPSC Differentiation Potential. Stem Cell Reports 2016, 6, (2), 200-12.

111. Goldberg, M. S.; Fleming, S. M.; Palacino, J. J.; Cepeda, C.; Lam, H. A.; Bhatnagar, A.; Meloni, E. G.; Wu, N.; Ackerson, L. C.; Klapstein, G. J.; Gajendiran, M.; Roth, B. L.; Chesselet, M. F.; Maidment, N. T.; Levine, M. S.; Shen, J., Parkin-deficient mice exhibit nigrostriatal deficits but not loss of dopaminergic neurons. J Biol Chem 2003, 278, (44), 43628-35.

112. Catic, A., Cellular Metabolism and Aging. Prog Mol Biol Transl Sci 2018, 155, 85-107.

113. Meyer, J. N.; Leung, M. C.; Rooney, J. P.; Sendoel, A.; Hengartner, M. O.; Kisby, G. E.; Bess, A. S., Mitochondria as a target of environmental toxicants. Toxicol Sci 2013, 134, (1), 1-17.

114. Georgiou, A.; Demetriou, C. A.; Christou, Y. P.; Heraclides, A.; Leonidou, E.; Loukaides, P.; Yiasoumi, E.; Pantziaris, M.; Kleopa, K. A.; Papacostas, S. S.; Loizidou, M. A.; Hadjisavvas, A.; Zamba-Papanicolaou, E., Genetic and Environmental Factors Contributing to Parkinson's Disease: A Case-Control Study in the Cypriot Population. Front Neurol 2019, 10, 1047.

115. Commission, E. State of Health in the EU Cyprus Country Health Profile 2019. https://www.euro.who.int/_data/assets/pdf_file/0009/419454/Country-Health-Profile2019-Cyprus.pdf (05.03.2021),

116. Parnetti, L.; Gaetani, L.; Eusebi, P.; Paciotti, S.; Hansson, O.; El-Agnaf, O.; Mollenhauer, B.; Blennow, K.; Calabresi, P., CSF and blood biomarkers for Parkinson's disease. Lancet Neurol 2019, 18, (6), 573-586.

117. Franz, D.; Olsen, H. L.; Klink, O.; Gimsa, J., Automated and manual patch clamp data of human induced pluripotent stem cell-derived dopaminergic neurons. Sci Data 2017, 4, 170056.

118. Koppensteiner, P.; Boehm, S.; Arancio, O., Electrophysiological profiles of induced neurons converted directly from adult human fibroblasts indicate incomplete neuronal conversion. Cell Reprogram 2014, 16, (6), 439-46.

119. Garcia Ruiz, P. J., [Prehistory of Parkinson's disease]. Neurologia 2004, 19, (10), 7357.

120. Lees, A. J., Unresolved issues relating to the shaking palsy on the celebration of James Parkinson's 250th birthday. Mov Disord 2007, 22 Suppl 17, S327-34. 


\section{Appendix}

\subsection{Publication I}

\section{Declaration of author contributions}

Title: Progression of pathology in PINK1-deficient mouse brain from splicing via ubiquitination, ER stress, and mitophagy changes to neuroinflammation

Status: published

Journal: Journal of Neuroinflammation, 2017, doi: 10.1186/s12974-017-0928-0

Contributing authors: Sylvia Torres-Odio (STO ${ }^{*}$, Jana Key $(\mathrm{JK})^{*}$, Hans-Hermann Hoepken $(\mathrm{HHH})$, Júlia Canet-Pons (JCP), Lucie Valek (LV), Bastian Roller (BR), Michael Walter (MW), Blas Morales-Gordo (BMG), David Meierhofer (DM), Patrick N. Harter (PNH), Michel Mittelbronn (MM), Irmgard Tegeder (IT), Suzana Gispert (SG) and Georg Auburger (GA)

* shared first authorship

What are the contributions of the doctoral candidate and her co-authors?

\section{(1) Concept and design}

Doctoral candidate (JK): $55 \%$

Co-author STO: $20 \%$

Co-author SG: $10 \%$

Co-autor GA: $15 \%$

\section{(2) Conducting tests and experiments}

Doctoral candidate (JK): $60 \%$ (expression analyses, biochemical characterization, cell culture experiments, immunostaining)

Co-author STO: 35\% (expression analyses, biochemical characterization, cell culture experiments, immunostaining)

Co-author SG: 5\% (mouse genetics, tissue collection)

\section{(3) Compilation of data sets and figures}

Doctoral candidate (JK): $\mathbf{5 0 \%}$ (expression analyses, biochemical characterization, immunostaining)

Co-author STO: $\mathbf{4 0 \%}$ (expression analyses, biochemical characterization, summary figure)

Co-author SG: $10 \%$ (mouse genetics)

\section{(4) Analysis and interpretation of data}

Doctoral candidate (JK): $\mathbf{5 5 \%}$ (biochemical characterization, expression analyses, immunostaining)

Co-author STO: $25 \%$ (biochemical characterization, expression analyses)

Co-author SG: 10\% (mouse genetics)

Co-autor GA: 10\% (interpretation of all data) 
(5) Drafting of manuscript

Doctoral candidate (JK): $\mathbf{5 0 \%}$

Co-author STO: $\mathbf{3 0 \%}$

Co-author SG: $\mathbf{5 \%}$

Co-autor GA: $\mathbf{1 5 \%}$

Date and place

Signature of the doctoral candidate

Date and place

Signature of the supervisor 


\section{Progression of pathology in PINK1-deficient mouse brain from splicing via ubiquitination, ER stress, and mitophagy changes to neuroinflammation}

Sylvia Torres-Odio ${ }^{1 \dagger}$, Jana Key ${ }^{1 \dagger}$, Hans-Hermann Hoepken ${ }^{1}$, Júlia Canet-Pons ${ }^{1}$, Lucie Valek², Bastian Roller ${ }^{3}$, Michael Walter ${ }^{4}$, Blas Morales-Gordo ${ }^{5}$, David Meierhofer ${ }^{6}$, Patrick N. Harter ${ }^{3}$, Michel Mittelbronn ${ }^{3,7,8,9,10}$, Irmgard Tegeder ${ }^{2}$, Suzana Gispert ${ }^{1}$ and Georg Auburger ${ }^{1 *}$

\section{Abstract}

Background: PINK1 deficiency causes the autosomal recessive PARK6 variant of Parkinson's disease. PINK1 activates ubiquitin by phosphorylation and cooperates with the downstream ubiquitin ligase PARKIN, to exert quality control and control autophagic degradation of mitochondria and of misfolded proteins in all cell types.

Methods: Global transcriptome profiling of mouse brain and neuron cultures were assessed in protein-protein interaction diagrams and by pathway enrichment algorithms. Validation by quantitative reverse transcriptase polymerase chain reaction and immunoblots was performed, including human neuroblastoma cells and patient primary skin fibroblasts.

Results: In a first approach, we documented Pink1-deleted mice across the lifespan regarding brain mRNAs. The expression changes were always subtle, consistently affecting "intracellular membrane-bounded organelles". Significant anomalies involved about 250 factors at age 6 weeks, 1300 at 6 months, and more than 3500 at age 18 months in the cerebellar tissue, including Srsf10, Ube3a, Mapk8, Creb3, and Nfkbia. Initially, mildly significant pathway enrichment for the spliceosome was apparent. Later, highly significant networks of ubiquitin-mediated proteolysis and endoplasmic reticulum protein processing occurred. Finally, an enrichment of neuroinflammation factors appeared, together with profiles of bacterial invasion and MAPK signaling changes—-while mitophagy had minor significance.

Immunohistochemistry showed pronounced cellular response of Iba1-positive microglia and GFAP-positive astrocytes; brain lipidomics observed increases of ceramides as neuroinflammatory signs at old age.

In a second approach, we assessed PINK1 deficiency in the presence of a stressor. Marked dysregulations of microbial defense factors Ifit3 and Rsad2 were consistently observed upon five analyses: (1) Pink $1^{-/-}$primary neurons in the first weeks after brain dissociation, (2) aged Pink $1^{-/-}$midbrain with transgenic A53T-alpha-synuclein overexpression, (3) human neuroblastoma cells with PINK1-knockdown and murine Pink $1^{1--}$ embryonal fibroblasts undergoing acute starvation, (4) triggering mitophagy in these cells with trifluoromethoxy carbonylcyanide phenylhydrazone (FCCP), and (5) subjecting them to pathogenic RNA-analogue poly(I:C). The stress regulation of MAVS, RSAD2, DDX58, IFIT3, IFIT1, and LRRK2 was PINK1 dependent. Dysregulation of some innate immunity genes was also found in skin fibroblast cells from PARK6 patients.

(Continued on next page)

\footnotetext{
* Correspondence: auburger@em.uni-frankfurt.de

${ }^{\dagger}$ Equal contributors

${ }^{1}$ Experimental Neurology, Goethe University Medical School, 60590 Frankfurt

am Main, Germany

Full list of author information is available at the end of the article
}

\section{Biomed Central}

( ) The Author(s). 2017 Open Access This article is distributed under the terms of the Creative Commons Attribution 4.0 International License (http://creativecommons.org/licenses/by/4.0/), which permits unrestricted use, distribution, and reproduction in any medium, provided you give appropriate credit to the original author(s) and the source, provide a link to the Creative Commons license, and indicate if changes were made. The Creative Commons Public Domain Dedication waiver (http://creativecommons.org/publicdomain/zero/1.0/) applies to the data made available in this article, unless otherwise stated. 
(Continued from previous page)

Conclusions: Thus, an individual biomarker with expression correlating to progression was not identified. Instead, more advanced disease stages involved additional pathways. Hence, our results identify PINK1 deficiency as an early modulator of innate immunity in neurons, which precedes late stages of neuroinflammation during alpha-synuclein spreading.

Keywords: Parkinson's disease, Ubiquitin kinase PINK1, Mitochondrial dysfunction, Antiviral response, Neuroinflammation

\section{Background}

Parkinson's disease (PD) is the second most prevalent neurodegenerative disease after Alzheimer's disease (AD). An autosomal recessive variant of PD, PARK6, is caused by loss-of-function mutations in PINK1 (PTEN-induced kinase 1) [1]. PINK1 activates ubiquitin by phosphorylation [2-4] and exerts quality control over mitochondria, controlling their translational repair, fusion, and elimination by mitophagy [5-9]. In this role, it cooperates with the downstream ubiquitin ligase PARKIN, which is responsible for another autosomal recessive variant of $\mathrm{PD}$, named PARK2 [10-12].

Independent from their role for mitochondria, PINK1 and PARKIN are transcriptionally induced by trophic and nutrient deprivation stress $[13,14]$, and they influence the trophic cell state through modulation of the signaling from glial cell line-derived neurotrophic factor (GDNF) and its receptor tyrosine kinase RET [15-17]. Unexpectedly, PARKIN was recently discovered to modulate also the cellular resistance against microbial invasion [18].

Thus, in spite of the deep knowledge about the roles of PINK1 and PARKIN for selective mitophagy, there is an important need to conduct unbiased surveys to elucidate additional functions of PINK1 and PARKIN in stress responses. Our previous OMICS work observed only mild changes in mRNA, protein abundance and posttranslational modifications in response to PINK1 loss-of-function, failing to identify individual biomarkers that correlate with disease progression [19-22].

Transcriptome dysregulations below 1.5 -fold are usually regarded with skepticism and are difficult to validate with other experimental techniques. However, in ageassociated neurodegenerative disorders, they cannot be disregarded. It is known that the 1.5-fold increase of beta-amyloid precursor protein (APP) dosage on chromosome 21 leads to typical AD symptoms and neuropathology already by the age of 40 years in most Down syndrome cases [23], so the triggers of any AD manifestation at ages 60-90 must be equivalent to an APP gene dosage much smaller than 1.5-fold. Similarly, the manifestation of PD is triggered by alpha-synuclein around age 35 years via a twofold gene dosage increase [24], around age 50 via a 1.5-fold gene dosage [25], and around age 70 by a 1.3 -fold gene dosage [26, 27]. In practically all chromosomal trisomies, the 1.5 -fold increase in dosage of some genes results in embryonal lethality, so any old-age pathology triggered from these genes would result from $<1.5$-fold expression dysregulation.

The relevance of subtle expression changes has been taken into account by modern analysis tools based on "gene set enrichment analyses" [28]. Therefore, we now reinvestigated our global transcriptome data with automated biomathematics tools, accepting that some false positive and false negative results will have to be dealt with, but hoping that the significant enrichment of pathways and subcellular compartments will identify PINK1-deficiency effects that are validated by the consistency over time as well as across tissues and species.

For this aim, we (1) analyzed the global transcriptome profile of Pink $1^{-/-}$mouse brain tissue at three ages, (2) surveyed Pink1-dependent regulations of the global transcriptome in neuron-rich primary cultures from postnatal mouse brain at 12 days after the acute brain dissection stress, (3) validated the results in aged $\operatorname{Pink} 1^{-/-}$brain where the transgenic overexpression of A53T-alphasynuclein (gene symbol SNCA) exerted chronic neurotoxic stress, (4) tested the cellular response of microglia and astrocytes in Pink $1^{-1-}$ brain by immunohistochemistry, (5) used lipidomics to study pro-inflammatory signals, (6) performed a systematic assessment of the expression of key factors of antiviral state in human neuroblastoma cells with lentiviral PINK1-Knock-Down (KD), studying the time course after acute starvation stress, (7) reassessed the same key factors of antiviral state in human PINK1-(KD) neuroblastoma versus $P i n k 1^{-1-}$ murine embryonal fibroblasts after mitophagy via FCCP drug treatment, and (8) tested the same factors after stress with the pathogenic poly(I:C) RNA regarding PINK1-dependent expression regulation. Primary skin fibroblasts from three patients at advanced age with manifest PD due to G309DPINK1 mutations were employed to assess the relevance of these data for the human disease.

\section{Methods}

\section{Mouse breeding and brain dissection}

Pink $1^{-/-}$and wildtype (WT) control mice, which were derived from common ancestors and share the strain $129 / \mathrm{SvEv}$ genetic background, were bred and genotyped as previously reported [29]. Brain tissue from Pink $1^{-/-}$ 
+A53T-SNCA double mutant mice was obtained as published [20].

\section{Global transcriptomics}

Affymetrix oligonucleotide microarray profiling was performed with Genechip mouse genome 4302.0 arrays as previously [30, 31], using cRNA from the brain cerebellar tissue as reported before [20] and from neuron-rich primary cultures from 3 Pink $1^{-1-}$ versus 3 age and sexmatched WT control mice. Hybridization occurred on Affymetrix Genechip mouse genome 4302.0 arrays, which represent 39,000 transcripts, of which more than half are anonymous or poorly understood, according to PubMed and GeneCards database searches. The biomathematical analysis was performed in the institute for medical genetics at Tuebingen University.

\section{Bioinformatic analyses}

For protein-protein interaction (PPI) network analysis, the software tool String v.10 (https://string-db.org/) with standard settings has been employed to visualize networks of significant dysregulations [32]. As recommended, gene symbols of factors with significant dysregulation were entered into the Multiple Proteins window with the Mus musculus option, the matching of the input with the correct factors was accepted, and the graphic interaction diagram was generated and archived. The Analysis button was used to generate automated network statistics; significant functional enrichments of GO (Gene Ontology) terms and KEGG pathways were exported into EXCEL files.

For an additional comprehensive transcriptome analysis, gene set enrichment analysis (GSEA, v2.2.3, http://software.broadinstitute.org/gsea/index.jsp) [28] was applied in order to see, if a priori defined sets of genes show statistically significant, concordant differences between mutant/WT in 18 months old cerebellum samples. For every gene, only the one entry with the lowest adjusted $p$ value and the according $\log _{2}$ transformed ratio was taken. GSEA default settings and Reactome v5.2 and KEGG v5.2 gene set database were used. Pathways with $p$ value $\leq 0.05$ and FDR $q \leq 0.25$ were regarded as significant. Heat maps were produced with the Perseus software.

\section{Primary neuron culture}

Neuron-rich primary cultures from the dissociated brain cerebral cortex of postnatal mice were prepared as previously described [33]. In short, 500,000 cells per well were seeded on a $0.01 \%(w / v)$ poly-D-lysine coated 6 well plate. In order to limit the growth of dividing cells, cytosine $\mathrm{B}$-D-Arabinoside was added on the second day of culture as before. The 3 plate pairs (mutant versus WT), where many singular neurons in homogeneous density with a dense network of processes contrasted with very few astrocytes and microglia present, were chosen out of a total of 12 plate pairs on culture day 12 for RNA extraction.

\section{Brain homogenate from aged mice}

Mouse aging and dissection of mice was carried out as before, employing cerebellum from single mutant Pink $1^{-/-}$ at three ages (10 mutants versus $10 \mathrm{WT})$ and midbrain from adult double mutant Pink $1^{-1-}+$ A53T-SNCA mice at age 18 months ( 5 mutants versus $5 \mathrm{WT}$ ) for the extraction of global RNA and cDNA synthesis [20].

\section{GFAP and lba1 immunohistochemistry}

Immunohistochemistry was performed using an automated staining system (Leica Bond-III, Nussloch, Germany). The following antibodies were used: anti-GFAP, rabbit polyclonal antibody, dilution 1:14,000 (DakoCytomation, Glostrup, Denmark); anti-Iba1, rabbit antibody, dilution 1:1000 (Wako Pure Chemical Industries, Osaka, Japan).

Representative areas of striatum, substantia nigra, neocortex, and brainstem of $\operatorname{Pink} 1^{-/-}(n=1)$ and wildtype $(n=1)$ animals were analyzed using ImageJ software (Version $1.51 \mathrm{~h}$; National Institutes of Health, Bethesda, Maryland, USA). We quantified positive cells in relation to all cells.

\section{Tissue preparation for lipid analysis}

Matched pairs of male and female Pink $1^{-/-}$and Pink $1^{+/+}$ mice were used for analysis of bioactive lipids in brain tissue. Mice were 9-13 months (four each), 17 months (three each), and 21 months (four each) old at the time of tissue preparation (mean age $15.5-/-$ and $17+/+$ ). Mice were sacrificed by carbon dioxide. Blood was drawn into $\mathrm{K}+$ EDTA microvettes (Sarstedt) for plasma analysis by cardiac puncture. Subsequent intracardial perfusion with saline removed rests of blood. The lumbar spinal cord, olfactory bulb, and hippocampus were dissected, and tissue pieces of 3-5 mg were excised, rapidly frozen in liquid nitrogen, and stored at $-80{ }^{\circ} \mathrm{C}$ until analysis. The precise tissue weight was determined on precision scales directly before tissue homogenization.

\section{Analysis of lipid signaling molecules}

Sphingolipids were analyzed by liquid chromatography tandem mass spectrometry (LC-MS/MS) in different regions of the nervous system (olfactory bulb, hippocampus, spinal cord) at three different ages (8.5-12.5 weeks, 17.5 weeks, and 21 weeks). LC-MS/MS analyses were done on an API4000 triple quadrupole mass spectrometer equipped with an APCI (atmospheric pressure chemical ionization) ion source for the analysis of ceramides and with an ESI (Electrospray Ionization) ion source for the analysis of sphingosines, Glu-Cer/Lac-Cer (Sciex, Darmstadt, Germany) [34, 35]. All quadrupoles 
were working at unit resolution. Concentrations of the calibration standards, quality controls, and samples were evaluated by MultiQuant 3.0 (Sciex, Darmstadt, Germany) using the internal standard method (isotopedilution mass spectrometry). Calibration curves were calculated by linear regression with $1 / x$ weighting. The coefficient of correlation for all measured sequences was at least 0.99 .

Tissue pieces of approximately $4 \mathrm{mg}$ were homogenized in $200-\mu \mathrm{l}$ extraction buffer $(50 \mu \mathrm{l}$ per $\mathrm{mg})$. For analysis of sphingolipids, $20 \mu \mathrm{l}$ plasma or homogenized tissue samples consisting in $0.4 \mathrm{mg}$ tissue were extracted with methanol:chloroform: $\mathrm{HCl}$ (15:83:2) after spiking with the respective internal standards, which was Cer17:0 for ceramides and sphingosine-D7 and sphingosine-1-phosphate-D7 for sphingosines. A Luna C18 column $(150 \mathrm{~mm} \times 2 \mathrm{~mm}$ ID, $5 \mu \mathrm{m}$ particle size, $100 \AA$ pore size; Phenomenex, Aschaffenburg, Germany) was used for chromatographic separation. The HPLC mobile phases consisted of water-formic acid (100:0.1, v/v) (A) and acetonitriletetrahydrofuran-formic acid (50:50:0.1, $v / v / v)$ (B). For separation, a gradient program was used at a flow rate of $0.3 \mathrm{ml} / \mathrm{min}$. The initial buffer composition was $60 \%$ (A) $/ 40 \%$ (B). It was maintained for $0.6 \mathrm{~min}$, then linearly changed to $0 \%(\mathrm{~A}) / 100 \%$ (B) over $3.9 \mathrm{~min}$, and held for $6.5 \mathrm{~min}$. Subsequently, the ratio was linearly changed back within $0.5 \mathrm{~min}$ to $60 \%$ (A) $/ 40 \%$ (B) and then held for another $4.5 \mathrm{~min}$. The running time for every sample was $16 \mathrm{~min}$. The injection volumes were $15 \mu \mathrm{l}$ for ceramides and $10 \mu \mathrm{l}$ for sphingosines. The analyses were done in multiple reaction monitoring (MRM) mode. For every analyte, two transitions were recorded, one for quantification and the other for identification. The peak area of the analyte was normalized for the peak area of the internal standard. Precursor to product ion transitions for quantification were: $m / z 539 \rightarrow 264$ for $C_{16: 0}$-Cer, $m / z 567 \rightarrow 264$ for $\mathrm{C}_{18: 0}$-Cer, $m / z 595 \rightarrow 264$ for $\mathrm{C}_{20: 0}$-Cer, $m / z 651 \rightarrow 264$ for $\mathrm{C}_{24: 0^{-}}$Cer, $m / z \quad 649 \rightarrow 264$ for $\mathrm{C}_{24: 1}$-Cer, $\mathrm{m} / z$ $700 \rightarrow 264$ for $\mathrm{C}_{16: 0^{-}}$GluCer, $m / z 729 \rightarrow 264$ for $\mathrm{C}_{18: 0^{-}}$ GluCer, $m / z 862 \rightarrow 264$ for $C_{16: 0}$-LacCer, $m / z 891 \rightarrow 264$ for C18:0-LacCer, $m / z 973 \rightarrow 264$ for C24:1-LacCer, $m / z$ $553 \rightarrow 264$ for C17:0-Cer, $m / z 300 \rightarrow 282$ for Sph and $m / z$ $380 \rightarrow 264$ for S1P. The dwell times were 15 or $50 \mathrm{~ms}$.

To assess genotype-dependent differences on individual ceramides in specific regions, mice of different ages were summarized. To assess progression over age, ceramides across regions were pooled, log2-transformed to linearize the data, and subsequently summed to get a global readout for all ceramides. Total ceramides were then plotted over time and genotype-dependent differences at different ages were analyzed using two-way ANOVA for "genotype x age".

\section{Neuroblastoma starvation}

The human SH-SY5Y neuroblastoma cell line with dopaminergic properties was stably transduced by lentivirus either with a control (NT for Non-Target knock-down) shRNA or a shRNA directed against PINK1 and maintained under puromycin $(1 \mu \mathrm{g} / \mathrm{ml})$ selection in RPMI medium containing 10\% Fetal Calf Serum (FCS), as published already [6]. These PINK1KD and NT control cell lines had the stability of their KD controlled repeatedly over many months. They were switched to HBSS medium without FCS, to subject them to a starvation time course as previously described [13].

\section{Quantitative reverse transcriptase real-time polymerase chain reaction (qPCR)}

RNA was isolated with the RNeasy mini kit (Qiagen) and then treated with DNase I. cDNA was synthesized with SuperScript III reverse transcriptase using oligo $(\mathrm{dT})_{20}$ and random primers (Invitrogen). cDNA from 20 to 25 ng RNA were utilized in a $20 \mu \mathrm{l}$ reaction volume using the StepOnePlus Real-Time PCR System and the appropriate murine (lowercase) or human (uppercase) TaqMan gene expression assays (Applied Biosystems): for mouse Pink1 (Mm00550827_m1), Creb3 (Mm00501607_m1), Ddx58 (Mm01216853_m1), Hebp1 (Mm00469161_m1), Ifit1 (Mm00515153_m1), Ifit3 (Mm01704846_s1), Irf3 (Mm00516784_m1), Mapk8 (Mm01218957_m1, Mm01218946_m1, Mm0048951 4_m1), Mapk9 (Mm00444239_m1), Mapk14 (Mm01 301009_m1), Mavs (Mm00523170_m1), Mfn1 (Mm00 612599_m1), Nfkbia (Mm00477798_m1), Rsad2 (Mm00 491265_m1), Srsf10 (Mm01193320_m1), Tbk1 (Mm004 51150_m1), Tnf (Mm00443258_m1), for human PINK1 (Hs00260868_m1), DDX58 (Hs01061436_m1), HEBP1 (Hs00211123_m1), IFIT1 (Hs03027069_s1), IFIT3 (Hs0 0155468_m1), IRF3 (Hs01547283_m1), LRRK2 (Hs004 11197_m1), MAVS (Hs00920075_m1), MFN1 (Hs0096 6851_m1), RSAD2 (Hs00369813_m1), SQSTM1 (Hs00 177654_m1), TBK1 (Hs00179410_m1). mRNA expression was normalized to the TATA binding protein gene expression or the Hypoxanthine Phosphoribosyltransferase 1 gene expression (Tbp: Mm00446973_m1, TBP: Hs99999910_m1, HPRT1: Hs99999909_m1). Relative expression changes were calculated with the $2^{-\Delta \Delta C t}$ method [36].

\section{Triggering mitophagy via treatment with FCCP}

The drug FCCP, which is known to uncouple the mitochondrial membrane gradient and trigger mitophagy [37], was administered over $24 \mathrm{~h}$ at $10 \mu \mathrm{M}$ concentration to human SH-H5Y neuroblastoma cells or murine embryonal fibroblasts, which had been cultured in DMEM plus $10 \%$ FCS and grown to 
confluency (approximately $4 \times 10^{6}$ cells) in T25 flasks as previously described [38]; then, the cells were collected, and the RNA was extracted with TRIzol methodology.

\section{Stressing cells with a pathogenic RNA-analogue}

The synthetic dsRNA polymer poly(I:C), which induces the RNA sensors that activate innate immunity [39, 40], was purchased from InvivoGen in the low molecular weight variant with the transfection agent LyoVec and used at a concentration of $1 \mu \mathrm{g} / \mathrm{ml}$ (for SH-SY5Y cells) or $2 \mu \mathrm{g} / \mathrm{ml}$ (for MEFs) as recommended by the manufacturer during $16 \mathrm{~h}$ before harvesting the cells and extracting RNA/protein.

\section{Human primary skin fibroblast cultures}

Previously established primary skin fibroblast cultures from 3 homozygous PARK6 patients (passages 12-14) were employed as published [12, 19, 41-43], in addition to one sex-/age-matched control (the principal investigator G.A., passage 14) and four matched control fibroblast lines from Coriell depository (catalog number AG02261/passage 6/age 61, AG06103/passage 16/age 29, AG06858/passage 5, age 47, AG12207/passage 14/age 68).

\section{Quantitative immunoblotting}

The isolation of total proteins from the primary skin fibroblasts was carried out as described [42]. Samples of $20 \mu \mathrm{g}$ were heated at $90{ }^{\circ} \mathrm{C}$ for $5 \mathrm{~min}$ and then separated in $10 \%$ tris-glycine polyacrylamide gels, using Precision Plus Protein $^{\text {Tm }}$ All Blue Standards as size marker. Transfer to nitrocellulose membranes (Protran, GE Healthcare) was done at $50 \mathrm{~V}$ for $90 \mathrm{~min}$, with blocking in 5\% BSA solution in $1 \mathrm{X}$ TBS-T for $1 \mathrm{~h}$ at room temperature (RT). Primary antibodies against LRRK2 (1:1000, NBP1-49954, Novus), IFIT3 (1:500, 15,201-1-AP, Proteintech), IFIT1 (1:500, 23,247-1-AP, Proteintech), DDX58 (1:700, 3743, Cell Signaling Tech), RSAD2 (1:500, 11,833-1-AP, Proteintech), and $\beta$-Actin (1:5000, A5441, Sigma-Aldrich) occurred in $1 \mathrm{X}$ TBS-T solutions overnight at $4{ }^{\circ} \mathrm{C}$. Fluorescent-labeled $\alpha$-mouse $(1: 15,000$, IRDye $800 \mathrm{CW}$, Li-Cor) and $\alpha$-rabbit (1:15,000, IRDye 680RD, Li-Cor) were the secondary antibodies. Fluorescence detection occurred on the Li-Cor Odyssey Classic Instrument.

\section{Statistical analyses}

Statistical significance was assessed using ANOVA or unpaired $t$ test with Welch's correction in the GraphPad Prism 5 software.

\section{Results}

Global transcriptome profile of $P i n k 1^{-/-}$mouse brain and its progression during aging

Previously, we documented the effects of PINK1 deficiency on brain regions such as midbrain, striatum, and cerebellum at different ages in the absence of stress or in the presence of A53T-alpha-synuclein overexpression as stressor, identifying marked effects of PINK1-deficiency on mitochondrial biology and excitability via global transcriptome profiles [20-22, 29, 44]. Irrespective of the size of fold-changes, we now document all significant expression dysregulations in Pink $1^{-/-}$brain, which were consistent (1) across the lifespan and (2) across diverse brain areas (Additional file 1: Table S1). As expected, the loss of Pink 1 transcript constituted the most significant effect, being detected by two microarray probesets. An additional downregulation was observed for the splicing repressor Srsf10 (also called Fusip1) (to 63\% on average), a stress response factor which regulates the levels of the inflammasome component Caspase 1 [45]. A downregulation was found also for the vesicle endocytosis factor Clta (to 32\%), which controls antibody isotype switching [46]. A converse upregulation was documented by two independent probesets for the splicing activator Srrm1 (to 187\%), which is responsible for splicing of the lymphocyte surface protein CD44 [47]. Another upregulation detected by two independent oligonucleotide probesets appeared for the precursor RNA processing factor Hnrnpr, which is known to regulate c-Fos and thus neuronal excitability, but also the expression of classical and non-classical MHC class I proteins (probeset 2610528B01Rik showing transcript elevation to $172 \%$ and probeset $\operatorname{Gm} 17388$ to $131 \%$ ) [48, 49]. Currently, there is almost no experimental evidence implicating PINK1 in splicing [22] or in endocytosis [50]. Against our expectations, these expression dysregulations did not increase with age, so the fold-changes at age 18 months were very similar to those at 6 weeks. Thus, the focus on the expression of individual transcripts failed to identify markers of pathology progression.

\section{Bioinformatic pathway analysis: Gene Ontology Enrichment by STRING}

To further elucidate the expression effects of PINK1deficiency in nervous tissue, we now considered the enrichment of functional pathways and of protein interaction networks, employing automated bioinformatics tools. This approach was focused on the cerebellum, in view of several advantages: (1) The cerebellum is relatively big and easy to dissect with negligible anatomical variance; (2) its expression changes will not get diluted as in other brain regions where substantial neuron population heterogeneity renders many effects minimal upon mixed tissue analysis; and (3) it does not suffer from the neuron loss and astrogliosis, which are 
expected to occur in the vulnerable brain regions of PD models and which would distort expression profiles.

To illustrate the impact of PINK1 deficiency on specific gene networks within the global cerebellar transcriptome and to visualize the progression from age 6 weeks via 6 months until age 18 months, we produced a STRING diagram of protein-protein interaction clusters for each age (Additional file 2: Fig. S1A-S1C). In these figures, it is evident that the progression of pathology involves progressively increasing numbers of individual factors and pathways, so that at age 18 months, only the most severe effects could be visualized.

In mice at age 6 weeks, around 250 factors showed significant expression changes (with false discovery rate, FDR, adjusted $p$ value below 0.05) in Affymetrix oligonucleotide microarray studies (Additional file 3: Table S2A, first datasheet). Their analysis regarding the enrichment of specific pathways and protein-protein interactions with the automated bioinformatics tools at the STRING webserver detected significant dysregulation for 158 molecules within the Gene Ontology (GO) Cellular Component term "intracellular membrane-bounded organelle" (FDR $q$ value $=6.39 \mathrm{e}-08$ ) (Additional file 3: Table S2B), in good consistency with the known mitochondrial localization of PINK1 protein during stress. Interestingly, an enrichment was notable for the KEGG pathway "Spliceosome" $(q$ value $=0.04$ only, in view of the few dysregulations at this initial stage of pathology) (a blown-up interaction diagram detail around the splicing factor Sfrs10 is shown in Fig. 1a). The STRING diagram (Fig. 1a, Additional file 2: Fig. S1A), which represents this age, shows the intracellular membrane-bounded organelle factors highlighted in red color.

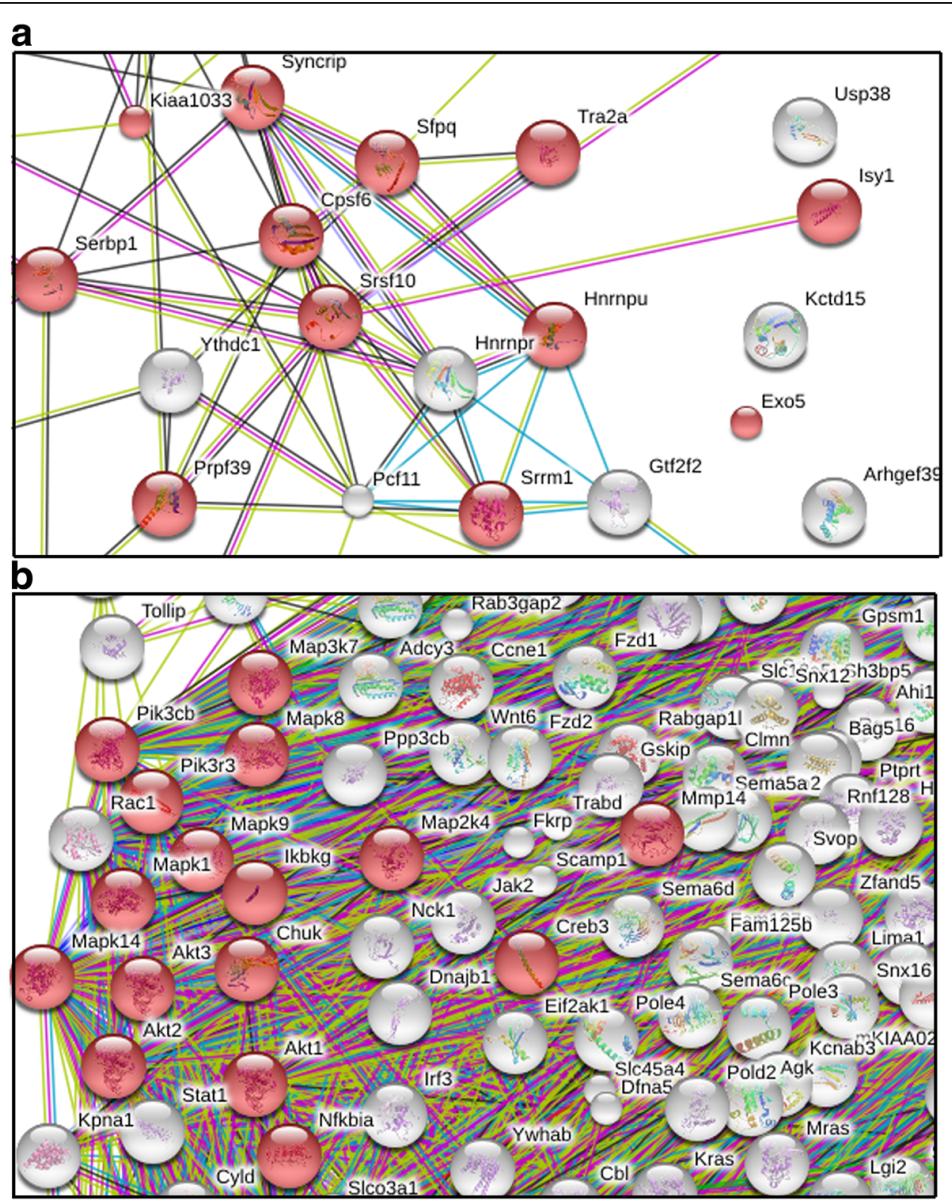

Fig. 1 Details from the STRING interaction diagrams within the global transcriptome profiles of Pink1-deficient mouse cerebellar tissue. a At age 6 weeks, a network of splicing factors around Srsf10 was dysregulated. Red symbols indicate membrane-associated factors. The complete profile interaction diagram can be found in Additional file 2: Fig. S1A. b At age 18 months, a much denser network including inflammatory factors like Creb3, Iff3, Nfkbia, Mapk8, Mapk9, and Stat1 was dysregulated. Components of the "Toll-like receptor signaling pathway" factors are highlighted in red color. The complete profile interaction diagram can be found in Additional file 2: Fig. S1C 
In mice at age 6 months, over 1300 factors showed expression changes at this significance threshold (Additional file 3: Table S2A, second datasheet). Their bioinformatics analysis detected significant dysregulation for 826 molecules within the GO Cellular Component term "intracellular membrane-bounded organelle" ( $q$ value $=7.61 \mathrm{e}-16$ ) (Additional file 3: Table S2C). Significant enrichment in the KEGG pathways "Ubiquitinmediated proteolysis" $(q=1.01 \mathrm{e}-05)$ and "Protein processing in endoplasmic reticulum" ( $q=3.34 \mathrm{e}-04)$ were notable. The corresponding STRING protein interaction diagram (Additional file 2: Fig. S1B) for this age again shows the intracellular membrane-bounded organelle factors highlighted in red color.

In mice at age 18 months, about 3500 factors showed expression changes at this significance threshold, too many to be processed by STRING bioinformatics (its limit is at 2000 nodes). Thus, only the about 1600 dysregulated factors with an adjusted $p$ value below 0.01 were analyzed regarding interactions and enrichments (Additional file 3: Table S2A, third datasheet). Significant enrichment for 1031 molecules within the GO Cellular Component term intracellular membrane-bounded organelle ( $q$ value $6.34 \mathrm{e}-67)$ was detected (Additional file 3: Table S2D). At this stage of progression, significant enrichment was observed in several GO biological processes (Additional file 3: Table S2E), particularly 537 factors in "cellular response to stimulus" ( $q$ value $2.82 \mathrm{e}-14), 165$ factors in "cellular response to stress" ( $q$ value $1.02 \mathrm{e}-09$ ), and 23 factors in "regulation of mitophagy" ( $q$ value 0.038$)$. These data are in agreement with the notion that the well-documented role of PINK1 in mitophagy is a small part of a much broader role of PINK1 in stimulus-dependent signaling and in stress responses. Significant enrichment was also observed in multiple KEGG pathways (Additional file 3: Table S2F), prominently for 47 factors in the "MAPK signaling pathway" ( $q$ value $2.64 \mathrm{e}-06$ ), for 32 factors in the "ubiquitin-mediated proteolysis pathway" ( $q$ value $2.64 \mathrm{e}$ $-06)$, for 34 factors in the "Protein processing in endoplasmic reticulum" pathway ( $q$ value $1.68 \mathrm{e}-05)$, and for 20 factors in the "Bacterial invasion of epithelial cells" pathway ( $q$ value $6.37 \mathrm{e}-05$ ). Mildly significant was the enrichment of 21 factors of the "Dopaminergic synapse" pathway ( $q$ value 0.005), 16 factors of the "GABAergic synapse" pathway ( $q$ value 0.008 ), and 18 factors of the "Glutamatergic pathway" pathway ( $q$ value 0.015 ). These data are in excellent consistency with previously established roles of PINK1 as a component of MAPK signaling $[51,52]$, as an activator of PARKIN which prevents bacterial invasions [18], and as a modifier of dopaminergic, GABAergic, and glutamatergic signaling in the nigrostriatal pathway [44, 53-56]. The agreement of our bioinformatics analysis of the global transcriptome with previously published cell biology data provides credibility to this automated approach.

In the STRING interaction diagram of cerebellar transcriptome changes at age 18 months, the appearance of neuroinflammatory factors was highlighted, manually placing the components of the significantly enriched KEGG pathways "HTLV-I infection", "Toll-like receptor signaling”, "TNF signaling pathway", "Fc epsilon RI signaling", "T cell receptor signaling pathway", "RIG-1-like receptor signaling", "Hepatitis C", and "Influenza A" in the lower left corner and highlighting the Toll-like receptor signaling pathway factors in red color (Additional file 2: Fig. S1C). A detail of this interaction diagram with focus on the inflammatory factors Creb3, Irf3, Nfkbia, Mapk8, Mapk9, and Stat1 is shown in Fig. 1b.

Heat maps were then used to focus on prominent examples among the significantly altered pathways, representing each dysregulated component at the three ages, not only for the Parkinson-resistant cerebellar tissue but also for comparing the Parkinsonvulnerable midbrain/brainstem and striatum (Table 1). This approach visualizes the temporal and spatial appearance of pathology as well as the effect sizes in a color code, with deep red mirroring strong upregulations and deep blue shades for strong downregulations. It also illustrates the number of factors and possibly the relevance of each pathway. Although the effect of PINK1 on ubiquitin-mediated proteolysis and on mitophagy are excellently studied, providing proof of principle that these moderate changes of many pathway components are relevant, it was novel to detect a selective dysregulation in particular for $U b e 3 a$ that has a known role in the degradation of cytoplasmic misfolded proteins like alpha-synuclein [57-59], for Xiap with an established function in ubiquitination, mitochondrial apoptosis, and innate immunity [60], for Dnm1l (encoding DRP1) with previously defined effects on PINK1-dependent mitochondrial fission [7, 61], as well as for Slc6a1 as a GABA transporter known to be induced by neuroinflammation [62]. The regional comparison in Table 1 made it evident that PINK1deficiency-mediated downregulations of stress-response factors dominate in the PD-resistant cerebellar tissue, while the same factors show converse progressive upregulation in the PD-affected midbrain/brainstem tissue, e.g., Keap1, Mbtps1, Rad23a, Sec61a1, Hif1a, Pi4k2a, the autophagy factors Map1lc3b and Sqstm1. Further opposing regulations occurred for the ubiquitin-binding factor Tollip, which regulates Toll-like receptor signaling as well as the autophagic clearance of ubiquitin conjugates and protein aggregates [63]. This supersensitive upregulation in tissues, which are known to suffer PD-specific stress, might represent a cellular effort to compensate PINK1 deficiency further downstream. 
Table 1 Global transcriptome analysis with prominent pathway dysregulations upon STRING assessment at 3 ages in different brain regions of Pink $1^{-1-}$ mice, illustrated as heat maps

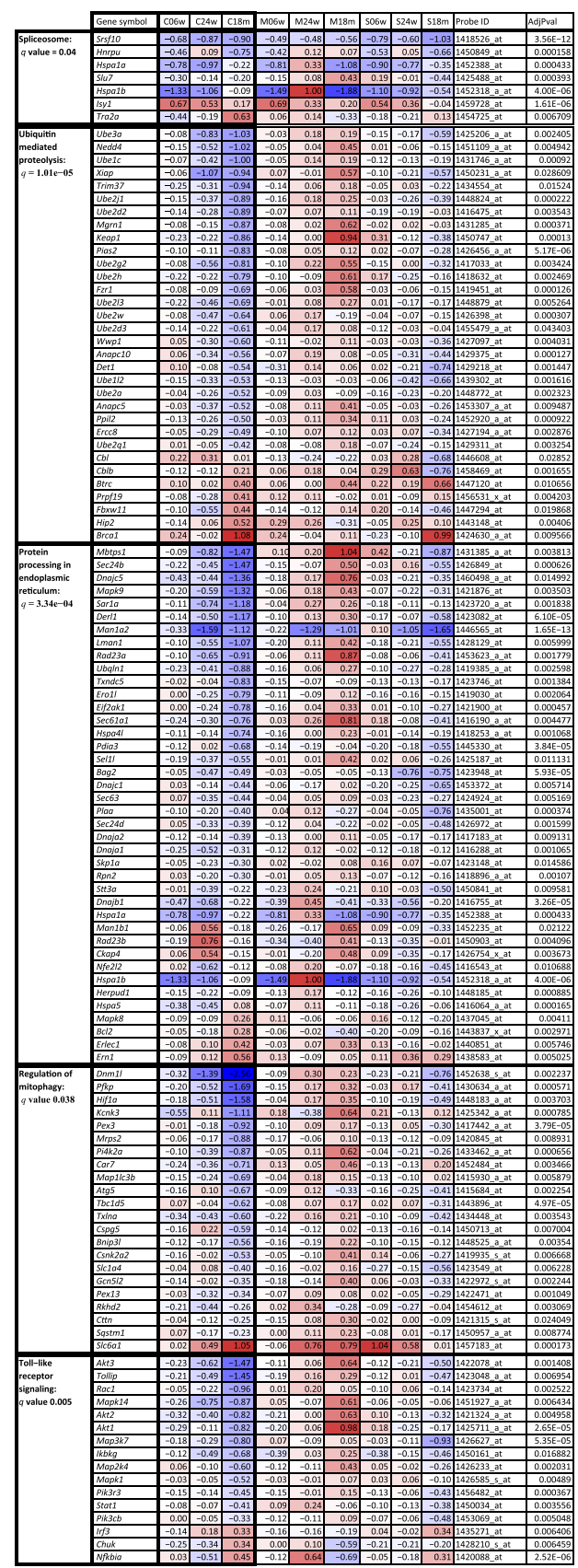

Expression dysregulations in Affymetrix microarrays are shown in different brain regions $(\mathrm{C}=$ cerebellum, $\mathrm{M}=$ midbrain, $\mathrm{S}=$ striatum) across the mouse lifespan ( $6 \mathrm{~W}=6$ weeks, $24 \mathrm{~W}=24$ weeks $=6$ months, $18 \mathrm{M}=18$ months), for genes detected by a specific oligonucleotide probe ID, together with the Adjusted $p$ values (AdjPval) as determined by Affymetrix transcriptome bioinformatics via software platform $\mathrm{R}$ and the bioconductor package Limma. Displayed are the genes contained in five exemplary pathways, which exhibited significant enrichment according to STRING bioinformatics, as indicated by the false discovery rate $q$ values. The ranked order of all dysregulated pathways upon STRING assessment can be found in Additional file 3: Table S2
If the STRING analysis is conducted with high stringency only for those cerebellar factors, which are significantly downregulated with $>1.5$-fold change, then much information is lost and the KEGG pathway of MAPK signaling becomes prominent at the different ages (Additional file 4: Table S3). This marked effect is not unexpected, since PINK1 (short for PTEN induced kinase 1) is downstream of PTEN, a phosphatase that antagonizes the kinase PI3K, which is an upstream modulator of the MAPK phosphorylation cascade at various levels. Furthermore, the interaction of PINK1 with the MAPK pathway has already been demonstrated experimentally in neuroblastoma cells, astrocytes, HeLa, and HepG2 liver cells $[51,64-66]$.

\section{Bioinformatic pathway assessment by Gene Set Enrichment analysis}

Going even further, instead of considering only the expression dysregulations beyond a significance threshold, we employed a tool that takes all genes with their expression ratios into account to compare them with known gene sets of functional relevance. This approach even recognizes compound effects, when most components of a pathway are subtly dysregulated in the same direction. This Gene Set Enrichment Analysis (GSEA) software thus performs a comprehensive assessment of the complete transcriptome data and is useful as an independent complement of the STRING approach. In the cerebellum, the most salient result was a significant effect for the KEGG pathway "Parkinson's disease", but this biomathematical finding was mainly due to the loss of Pink 1 transcript. Again, at the age of 6 weeks among the KEGG pathways, spliceosome downregulation appeared prominently (Additional file 5: Table S4). Downregulations of "Antigen processing and presentation" as well as "Ubiquitin-mediated proteolysis" were already significant. Among the Reactome pathways at this age, downregulations in the "innate immune system" already had nominal significance. At the age of 6 months, again an impairment of "endoplasmic reticulum stress responses" appeared with a significant downregulation of the Reactome pathway "Activation of chaperone genes by Xbp1s". At the age of 18 months, among the Reactome pathways, "antiviral mechanism by IFN stimulated genes" became prominent, and in particular the "Negative regulators of RIG I and MDA5 signaling" just touched significance in FDR. For the maximal pathology at the age of 18 months in the cerebellum, the top dozen dysregulated pathways from the KEGG database and the top dozen dysregulated pathways from the Reactome database were documented with individual components and their expression scores (Additional file 6: Table S5). Moreover, four Reactome pathways with special relevance for this manuscript were analyzed in their progressive 
dysregulation by heat maps (Additional file 7: Table S6). Thus, the temporal order of pathway involvement and the late-stage prominence of neuroinflammation were reproducible in an alternative approach.

\section{QPCR validation of candidate dysregulations in aged Pink $1^{-/-}$mouse cerebellum}

Experimental validation was performed for several dysregulated components of these pathways. As shown in Additional file 8: Fig. S2A, a significant downregulation was confirmed for the stress responsive splicing factor Srsf10 [67], while significant upregulations were observed for the cerebellar mRNA levels of Creb3 (human LZIP, synonymous LUMAN), which influences endoplasmic reticulum protein processing $[68,69]$, and for Nfkbia (NF-Kappa-B inhibitor alpha), which cooperates with $\mathrm{Creb3}$ to modulate the nuclear control of stress and inflammation responses [70-73], but not for other candidates within the Mapk (MAP kinase) gene family (Additional file 8: Fig. S2A). Of particular interest was the dysregulation of Ube3a within the ubiquitination pathway, because only one splice isoform changed its expression, which might be target of the spliceosome adaptation observed above, and because UBE3A is involved in the degradation of alpha-synuclein as the main driver of Parkinson pathogenesis [59].

We then focused on JNK1 (Mapk8 mRNA) expression, a crucial component of the stress-dependent phosphorylation cascades that control endoplasmic reticulum protein processing, neuroinflammation, and apoptotic or autophagic cell death [74-77]. JNK1 activity is also regulated by alternative splicing $[78,79]$; therefore, three representative exon-exon junctions were assessed. Quantitative reverse transcriptase PCR (qPCR) in Pink $1^{-1-}$ cerebellum at age 18 months in the absence of additional stressors demonstrated a significant but mild upregulation, 1.15-fold \pm 0.04 SEM with $p=0.01$ (Additional file 8: Fig. S2B). This finding is consistent with a previous report of increased p38 MAPK activation in PINK1-deficient mouse astrocytes [51]. However, the expression change of JNK1 was too subtle to be validated by quantitative immunoblots. This technique has inherent variability and limited linearity, so it requires an impractically high number of samples to detect 1.1-fold changes. Thus, a subtle dysregulation of the spliceosome pathway, the endoplasmic reticulum protein processing pathway, and neuroinflammatory response pathways was observable in global microarray transcriptome profiles upon unbiased bioinformatics analyses in old Pink $1^{-1-}$ mouse cerebellum, and the alteration of these pathways could be validated experimentally by qPCR for several key factors.

Overall, PINK1 deficiency triggered a dysregulated expression of many membrane-associated factors already in the first weeks of life. The progression of pathology occurred mainly through involvement of more pathways and protein interaction networks, rather than through stronger expression dysregulation. Pathological mitophagy and neuroinflammation profiles were apparent by the age of 18 months in Pink $1^{-1-}$ cerebellum.

\section{Activation of the cellular immune response in Pink $1^{-/-}$ mouse brain}

To test if the molecular profile of immune activation is reflected by cellular responses in the brain tissue, immunohistochemistry of GFAP as marker of astroglia and of Iba1 as marker of microglia was performed. Parallel processing of the sections was necessary to visualize their subtly increased immunoreactivity in diverse areas of the Pink $1^{-/-}$brain, which was prominent at the myelinated fiber tracts, e.g., the corticospinal projections through the striatum, the brainstem, and also in the cerebellar white matter (Fig. 2a, b). This increase was not detectable in nuclei like the striatal matrix or the substantia nigra and was not consistent in the cerebral cortex upon counting reactive cells in sections at even intervals throughout the brain (Fig. 2c). Thus, the dysregulation of membrane-associated factors in old animals leads to a cellular immune response most likely at myelinated axons.

\section{Accumulation of ceramides in Pink $1^{-1-}$ mouse brain}

To further assess the relevance of the subtle transcriptional changes at the effector molecule level, we decided to analyze ceramides of different chain lengths and saturation and their sugar-modified metabolites because ceramides trigger lethal mitophagy [80], lactosyl-ceramides maintain neuroinflammation [81], and ceramides accumulate upon lysosomal dysfunctions and LRRK2-deficiency [82] and are expelled by activated astrocytes via exosomes [83]. Hence, ceramide pathology may be a key factor for the progression of Parkinson's disease.

Ceramides, glucosyl-ceramides, and lactosyl-ceramides were increased in the $\operatorname{Pink} 1^{-/-}$olfactory bulb starting from 9 months on (Fig. 2d). Analysis of variance for repeated measurements revealed significant differences between genotypes $(F(1: 20)=7297 ; P=0.0137)$ and for the interaction "ceramide by genotype" $(F(10: 200)=4.558$; $P<0.0001)$. Subsequent post-hoc analyses for each ceramide and sphingolipid separately were significant for Cer18:0 $(P<0.0001)$, LacCer24:1 $(P=0.0285)$, and GluCer18:0 $(P=0.0042)$. For individual ceramides, there was no site-specific significant progression with increasing age, but analysis of log-2 transformed total ceramides revealed age-dependent differences. In wildtype animals, log-2 Cers linearly increased over time, but in Pink $1^{-/-}$, levels were already increased at 9 months and remained at this elevated level, so that genotype-dependent differences were strongest in young mice (2-way ANOVA "genotype $\mathrm{x}$ age" for genotype $F(1,54)=16.98, P=0.0001$; for the interaction $F(2,54)=2.531, P=0.089)$ (Fig. 2e). Hence, 


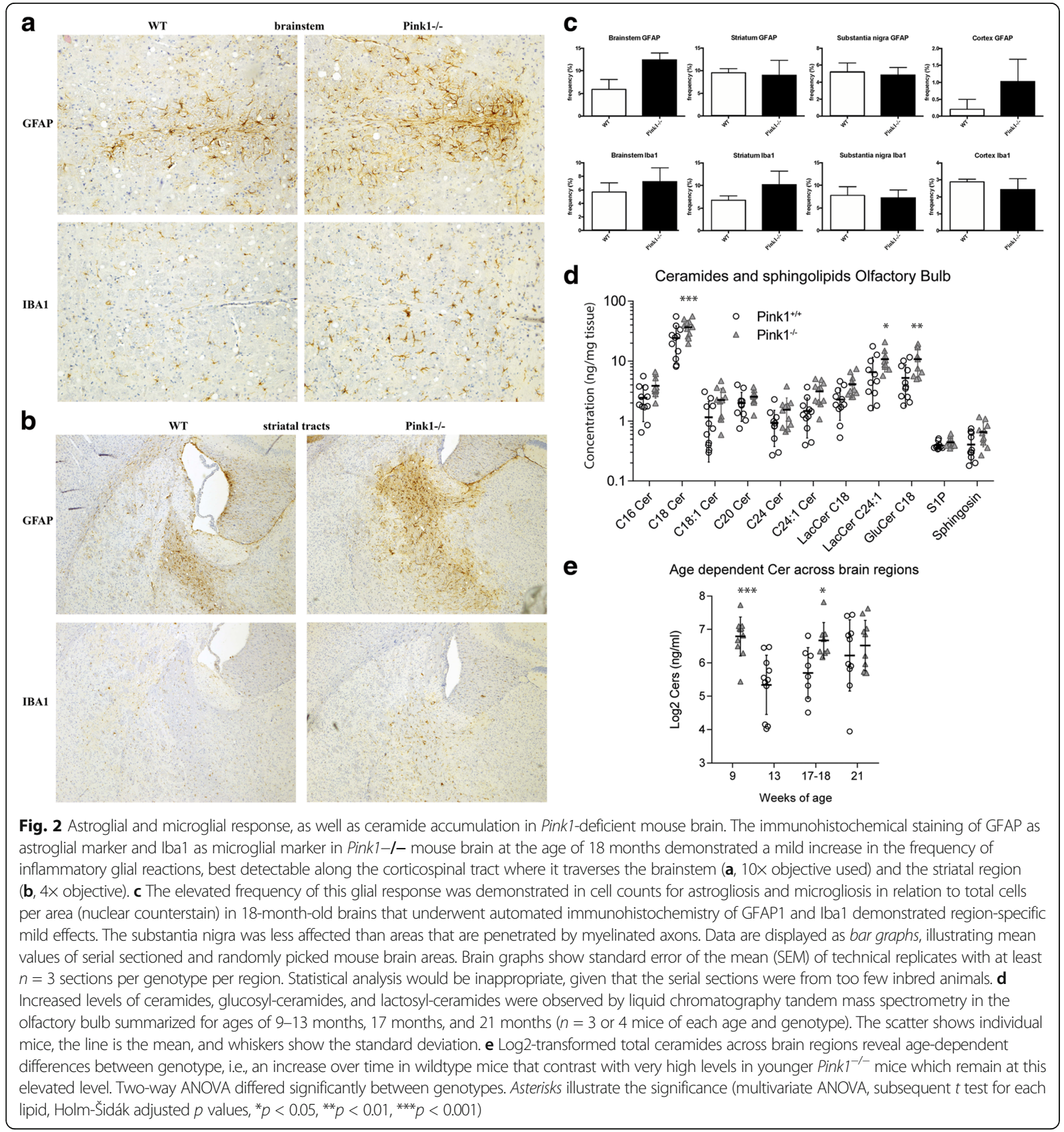

the observed increase of ceramide levels is strongly suggestive of ongoing neuro-inflammation in the brain starting in young adulthood, particularly in the olfactory bulb-a brain area which is among the first to be affected by Parkinson's disease.

\section{Global transcriptome profile of Pink $1^{-/-}$mouse neuron- rich primary cultures}

Given that PINK1 is important for stress responses $[5,6,13]$, and in view of the enhanced release of inflammatory cytokines from acutely prepared Pink1 -/- brain slices [84], we decided to study very young Pink $1^{-/-}$neuron-rich primary culture in vitro-where the previous mechanical dissection, the lack of glial support, and the presence of the toxin cytosine arabinoside (cytarabine) exert combined stress $[85,86]$. Hence, murine neuron-rich primary cultures were established at postnatal age and maintained for 12 days to assess early effects of PINK1 deficiency on global transcriptome readouts (Additional file 9: Table S7). Given that genomic insertion 
events may influence the expression of neighboring genes across a distance of 3 MegaBases [87], it was reassuring that only one dysregulated transcript, Nipal3, was encoded in the vicinity of Pink1.

Two findings had obvious credibility, but limited novelty, namely the dysregulation of Dram1 and Ret mRNA levels. Ordered by significance, the Dram1 (the abbreviation stands for DNA-damage regulated autophagy modulator 1) transcript 1.7-fold upregulation was the strongest early change after Pink1 loss ( $p$ value $\approx 0.0001)$. Similar to PINK1, DRAM1 is connected to the pathway of selective autophagy, since it promotes the targeting of mycobacteria to degradation [88], and is thus contributing to innate immunity responses. DRAM1 mRNA is selectively downregulated in brain tissue of PD patients [89]. A similarly meaningful observation was the 1.7 -fold upregulation of Ret (proto-oncogene RET receptor tyrosine kinase) ( $p$ value $\approx 0.001)$. RET trophic receptor signaling was already shown to rescue histological and biochemical phenotypes of Pink1 deletion in flies [15]. In a human neuroblastoma cell line, the administration of its receptor ligand GDNF compensates morphological and bioenergetic deficits of PINK1-deficient cells without affecting mitophagy. Furthermore, GDNF stimulation rescued mitochondrial defects in PARKIN-deficient cells, while the loss of dopaminergic midbrain neurons in aged RET-deficient animals was rescued by PARKIN overexpression and exacerbated by PARKIN deficiency [16]. Intriguingly, GDNF transfected macrophages significantly ameliorated neuroinflammation and neurodegeneration in a PD mouse model [90]. Thus, the Ret mRNA upregulation in Pink1-deficient primary neuron cultures after dissociation stress may also form part of the neuroinflammatory response pathway.

In addition to the known candidates, we observed a remarkable and novel enrichment of additional factors involved in antiviral state, innate immunity, and neuroinflammation (Additional file 9: Table S7) including Gin1 (gypsy retrotransposon integrase 1) [91], Hmox1 (Heme oxygenase 1) [92, 93], Hebp1 (Heme-binding protein 1) [94], Snx16 (Sorting nexin 16) [95], Timp1 (Tissue inhibitor of metalloproteinase 1) [96, 97], Dnmt2 (DNA methyltransferase 2) [98-101], Rsad2 (Radical S-adenosyl methionine domain containing 2 = Viperin) [102-106], Adamts4 (a disintegrin-like and metallopeptidase-reprolysin type-with thrombospondin type 1 motif, 4) [107-109], Csde1 (Cold shock domain containing E1, RNA binding = UNR) [110], and Ifit3 (interferon-induced protein with tetratricopeptide repeats 3) [111, 112].

Further enrichments in the transcriptome profile were apparent for DNA/RNA-quality control and for antioxidant/hypoxia factors. The DNA/RNA quality control factors included Zfp148, Cdk7, Tia1, Mbd1, Mus81, Mat2a, and Hipk2 [113-127]. The hypoxia/antioxidant factors, which are credible in view of the well-documented oxidative stress and bioenergetics deficit in PINK1deficient cells [21, 41], included Srxn1, Hlf, Steap1, Tsga10, and Hipk2 [126-132].

Intriguingly, Foxp1 (Forkhead Box P1) showed a 1.7-fold downregulation ( $p$ value $\approx 0.03$ ). This differentiation factor of midbrain dopaminergic neurons was previously found downregulated also in mice with knock-out of alpha-synuclein, while its upregulation was documented in mice with transgenic overexpression of A53T-alpha-synuclein, which model the PARK1 and PARK4 variants of PD [30, 31, 133]. Thus, the dysregulation of Foxp1 transcripts levels appears to respond both to acute stress and to constant mutations of at least two PD genes.

Overall, already at neonatal stages, a significant expression dysregulation of innate immunity factors in Pink $1^{-/-}$ primary neurons became apparent under in vitro stress conditions.

\section{Transcript analysis in the brain from adult Pink $1^{-/-}$mice with chronic A53T-SNCA stress}

For independent validation of these observations, and to exclude that a microbial infection of the cultures had produced artifacts, we next studied the transcript levels of candidate genes by qPCR in brain homogenate extracts from independent animals. Given that the stress-evoked observations in primary Pink $1^{-1-}$ neurons were not evident in global transcriptome screenings of single-mutant Pink $1^{-1-}$ mouse brains even at old age [20], we reasoned that additional challenges may be necessary to manifest PINK1-dependent stress response dysregulations. Thus, we studied double-mutant mice, where the Pink $1^{-/-}$is combined with 1.5-fold overexpression of A53T-alphasynuclein (SNCA) selectively in neurons as a trigger of Parkinsonian pathology and as a genetic interactor of PINK1 $[19,20]$. These double-mutant mice show a potentiated phenotype with appearance of Lewy-like inclusion bodies and with lethality from the age of 14 months onward [20]. The global transcriptome in several brain regions throughout their lifespan was previously compared to single mutant Pink $1^{-1-}$ mice, and the data are publically available in a database [20]. It has always been our experience that the heterogeneity of neuron populations in midbrain/brainstem and the dissection variance make it very difficult to demonstrate subtle expression changes.

In midbrain from adult double-mutant Pink $1^{-1-}+\mathrm{A} 53 \mathrm{~T}-$ SNCA mice at the age of 18 months, a significant 1.16-fold upregulation for Mapk9 ( $p$ value $=0.0007$ ), a significant 2.15 -fold upregulation for Rsad2 ( $p$ value $=0.0007$ ), a trend towards significance $(p$ value $=0.08)$ for the 0.87 -fold downregulation (a dysregulation so subtle that it would usually be deemed irrelevant) of Hebp1, and a significant 1.92-fold upregulation for Tnf alpha were observed $(p$ value $=0.03)($ see Additional file 10: Fig. S3 $)$. 
Thus, also in midbrain, the PINK1 deficiency led to dysregulated expression levels of key factors in the antimicrobial defense, when an additional challenge represented by mutant A53T-alpha-synuclein exceeded the allostatic threshold.

\section{Systematic study of antiviral factor mRNAs in human PINK1-knockdown neuroblastoma cells after acute starvation}

We decided to test in vitro whether (I) similar observations indeed occur in neural cells or have to be attributed to microglia, (II) the mouse data can be translated to human, (III) transient changes can be defined after acute stress, and (IV) trophic and nutrient deprivation stress with concomitant autophagy can trigger these stress response changes, always in dependence on PINK1 deficiency. The starvation stress was initially chosen rather than established Parkinsonian toxins like MPTP or CCCP $[6,134]$, since PINK1 was recently shown to respond to mutations in ATXN2, which is a known starvation response factor [135-138], and in view of the known transcriptional induction of PINK1 and PARKIN during trophic deprivation [13]. The human neuroblastoma cell line SH-SY5Y with stable lentiviral knockdown (KD) of PINK1 versus a non-target (NT) control sequence were subjected to acute starvation by changing the culture conditions from RPMI medium with $10 \%$ Fetal Calf Serum (FCS) to HBSS medium (low glucose, no amino acids) without FCS. The transcriptional regulation was documented over 2 days for several key factors of the antiviral defense by qPCR. The overview on this systematic survey of the mitochondria-associated antiviral defense pathway is represented in Fig. 3a, with blue color illustrating deficiency and PINK1-dependent downregulation, while red color illustrates PINK1-dependent upregulation. The KD was stable, since PINK1 mRNA in this cell line was reduced to $30 \%$ before stress and to $9 \%$ after stress at the 16-h time point (Fig. 3b). As described, the starvation induced PINK1 mRNA in a phasic manner with a peak at $12 \mathrm{~h}$ [13]. Similar to the previous findings in Pink $1^{-/-}$mice, an upregulation of RSAD2 and a downregulation of $H E B P 1$ were observed in the starving PINK1-deficient neuroblastoma cells. RSAD2 was induced up to 1.6-fold in control cells, and this response was potentiated by $46 \%$ in PINK1-KD cells, with strong significance at 2 and $4 \mathrm{~h}$ (Fig. 3c). The up to 3.3-fold induction of HEBP1 levels in control cells was significantly diminished at $16 \mathrm{~h}$ in PINK1-KD cells (Fig. 3d).

Systematic further assessment of the innate immunity pathway revealed further regulations, with PINK1deficiency modulating the alterations caused by starvation. A supersensitive transcriptional upregulation was observed not only for RSAD2 but also for TBK1 and IRF3. TBK1 was induced up to 1.9 -fold in control cells, and this induction was further increased to 2.2 -fold in
PINK1-KD cells, with strong significance at 4, 8, 24, and 48 h (Fig. 3e). Similarly, IRF3 levels were elevated up to 2.2-fold in control cells, and this elevation was exacerbated to 2.4-fold in PINK1-KD cells with strong significance at 24 and $48 \mathrm{~h}$ (Fig. 3f).

In contrast, a downregulation was observed for a known posttranslational modification target of PINK1/PARKIN signaling, mitochondrial MFN1. The 2.0-fold starvationtriggered induction of MFN1 transcript was blunted in PINK1-KD cells by $32 \%$, with strong significance at 12 and $16 \mathrm{~h}$ (Fig. 3g). Starvation triggered an initial short upregulation and later consistent downregulation of IFIT3 levels to $15 \%$ in control cells. In PINK1-KD cells, a strong downregulation of IFIT3 levels appeared already at $2 \mathrm{~h}$ and was stably diminished further to $6 \%$ of control at $48 \mathrm{~h}$, with significant differences between NT and PINK1-KD cells from 2 to $16 \mathrm{~h}$ (Fig. 3h). The starvation-triggered up to 1.7-fold induction of IFIT1 levels was also diminished in PINK1-KD cells by $41 \%$ with strong significance at 8,12 , and $16 \mathrm{~h}$ (Fig. 3i). Finally, the starvation-triggered almost 3.7 -fold induction of the mitochondria-associated innate immunity factor MAVS was blunted in PINK1-KD cells by $40 \%$, with strong significance at 12 and $16 \mathrm{~h}$ (Fig. 3j).

No significant dysregulation was documented for the transcript levels of TMEM173 (STING) as an endoplasmic reticulum-associated antiviral factor (data not shown). Given that experimental testing of multiple mRNAs at multiple time-points followed by statistical evaluation via Student's $t$ tests will exaggerate the significance, an adjustment of the significance threshold according to Bonferroni is useful, by dividing the nominal $p$ values indicated in each panel of Fig. 3 through the number of analyses done. After this correction, the downregulation of PINK1, MFN1, MAVS, IFIT1, and IFIT3 and the upregulation of $R S A D 2$ and $T B K 1$ remain significant as particularly robust findings.

In order to provide a time-correlation and comparison with autophagy factors that are also regulated by starvation, the curves for LRRK2 and SQSTM1 are shown as well (Fig. $3 \mathrm{k}-\mathrm{m}$ ), given that LRRK2 is a positive regulator of inflammation and autophagy, with mutations that trigger autosomal dominant Parkinson's disease; SQSTM1 (sequestosome $1=$ p62) was studied as an adaptor between the autophagy machinery and ubiquitinated cargo, whose mutation cause neurodegenerative disorders such as motor neuron disease. A significant indirect correlation was observed again between the PINK1-deficiency and an exacerbated induction of $L R R K 2$ transcript levels, as previously reported for fibroblasts and neuronal cells derived from PARK6 patients [139].

Overall, the data confirm that key factors of the antimicrobial response are modulated in their transcriptional regulation by PINK1 in human neural cells after acute starvation stress. 


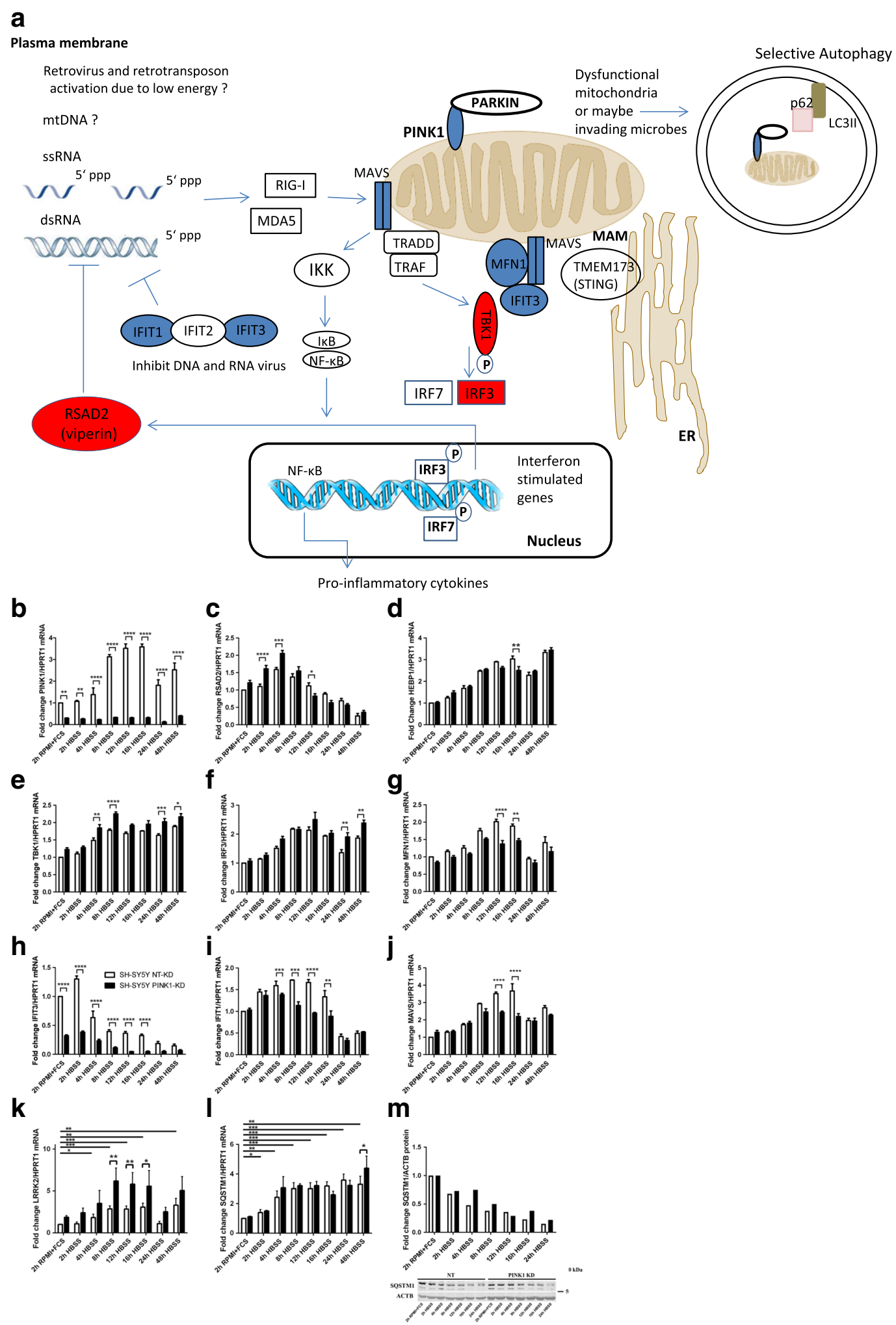

Fig. 3 (See legend on next page.) 
(See figure on previous page.)

Fig. 3 Systematic analysis of key factors in the mitochondria-associated innate immunity pathway, using acute starvation of human SH-SY5Y neuroblastoma cells to reveal the transcriptional regulation of stress responses and their dependence on PINK1. Transcript levels were documented in control non-target knock-down (NT-KD) versus PINK1-knock-down (PINK1-KD) cells for the following inflammatory factors. a Summary scheme on the detection of pathogen DNARRNA in the cytosol and the mitochondria-associated triggering of innate immunity, as altered during starvation by PINK1. Viral RNA (ssRNA or dsRNA) is recognized in the cytosol by helicase RIG-I and MDA5. This triggers the dimerization of MAVS in the outer mitochondrial membrane (OMM) which recruits transactivators (such as TRADD, TRAF, IKK family) leading to nuclear translocation of phosphorylated IRF3, IRF7, and NF-KB and promoting the transcription of IFN stimulated genes and pro-inflammatory cytokines, respectively. Effector molecules such as IFIT1, IFIT2, IFIT3, and RSAD2 (viperin) inhibit virus DNA/RNA replication. IFIT3 also functions as a scaffold to facilitate interaction between MAVS and TBK1 and represents a positive feedback of DDX58 (RIG-I) signaling through MAVS. Low $\triangle \psi_{\mathrm{m}}$ or decreased ROS inhibit MAVS-mediated signaling. Mitochondria cooperate with the endoplasmic reticulum (ER) to regulate lipid synthesis and antiviral signaling at the mitochondria-associated membranes (MAM), possibly by interactions of MAVS/MFN1 with TMEM173 (STING). MFN1 leads to the redistribution of MAVS along mitochondria and a fusion of the mitochondrial network that promotes the interaction between MAVS and STING. Low energy induces the localization of PINK1 to the OMM and recruitment of PARKIN from the cytosol, which is the signal for dysfunctional mitochondria to be digested in the autophagosome. Abbreviations: $5^{\prime}$ ppp 5' triphosphate, $\Delta \psi_{m}$ mitochondrial membrane potential, IkB inhibitor of $k$ light polypeptide gene enhancer in B cells, IKK IKB Kinase, LC3/l phosphatidylethanolamine conjugate of the autophagy-related protein LC3 (MAP1LC3), MDA5 melanoma differentiation-associated gene 5, NF-KB nuclear factor $\mathrm{KB}$, p62 sequestosome-1, adaptor between polyubiquitinated substrates and autophagic machinery, PARKIN ubiquitin ligase, its loss-of-function leads to the PARK2 variant of Parkinson's disease, R/G-1 (DDX58) retinoic acid-inducible gene-1 (DEXD/H-Box RNA Helicase 58), ROS reactive oxygen species, sSRNA dsRNA single-stranded/double-stranded RNA, TMEM173 (STING) transmembrane Protein 173, TRADD tumor necrosis factor receptor type 1 associated death domain protein, TRAF TNF-receptor-associated factor. b PINK1 (PTEN induced kinase 1) as a known determinant of selective mitophagy and autosomal recessive Parkinson's disease; HPRT1 (Hypoxanthine Phosphoribosyltransferase 1) as a loading control. c RSAD2 (= viperin, radical S-adenosyl methionine domain containing 2) as an interferon-inducible lipid-droplet associated virus inhibitory factor. $\mathbf{d}$ HEBP1 (heme binding protein 1) that contains a natural ligand for formyl peptide receptor-like receptor 2. e TBK1 (TANK-binding kinase 1) that phosphorylates interferon regulatory factors in response to toll-like receptor activation. $\mathbf{f} / R F 3$ (interferon regulatory factor 3 ) as a regulator of type I Interferon gene transcription. g MFN1 (mitofusin 1) as PARKIN-dependent factor in mitochondrial dynamics and mitochondria-associated anti-microbial signaling. $\mathbf{h}$ IFIT3 (interferon-induced protein with tetratricopeptide repeats 3) as a detector of pathogen DNA/RNA. i IFIT1 (interferon-induced protein with tetratricopeptide repeats 3) as a detector of pathogen DNARRA. j MAVS (mitochondria-associated viral sensor) as inducer of interferon-dependent long-term expression of defense factors. $\mathbf{k}-\mathbf{m}$ The levels of known autophagy factors during this starvation time course exhibited progressive consumption of p62 in spite of its transcriptional induction, in parallel to a PINK1-modulated transcriptional induction of LRRK2. Four independent experiments compared their expression during a nutrient and trophic deprivation time course triggered by a culture switch from RPMI growth medium to HBSS starvation medium. The bar graphs show mean and standard error of the mean, illustrating the significance with asterisks $\left({ }^{*} p<0.05,{ }^{* *} p<0.01,{ }^{* * *} p<0.001,{ }^{* * * *} p<0.0001\right)$

\section{Systematic study of antiviral factor mRNAs in human PINK1-knockdown neuroblastoma cells after triggering mitophagy by FCCP}

To further assess if indeed mitochondrial dysfunction underlies changes in innate immunity, we used the uncoupling drug FCCP to trigger mitophagy and then study the transcriptional response of the above key factors in PINK1-knockdown and control SH-SY5Y neuroblastoma cells. Mitophagy needs at least $12 \mathrm{~h}$ to occur and subsequent transcriptional responses require additional time. Therefore, the RNA extraction and qPCR analyses were performed later than in the previous experiment, at $24 \mathrm{~h}$ after drug administration. As shown in Additional file 11: Fig. S4 above, FCCP in SH-SY5Y cells elicited a transcriptional induction of the mitochondria associated factors PINK1, DDX58, and MAVS and of $T B K 1$, accompanied by a downregulation of the RNA sensor IFIT1, in control cells. A significant difference between control cells and PINK1 knockdown cells after FCCP treatment was only observed for MAVS, which failed to be induced in the absence of PINK1. These data confirm that key inflammatory factors respond to mitochondrial dysfunction and that particularly the levels of the mitochondrial antiviral signaling factor MAVS depend on PINK1 levels.

\section{Systematic study of antiviral factor mRNAs in Pink $1^{-/-}$murine embryonal fibroblasts after triggering mitophagy by FCCP treatment}

Pink $1^{-1-}$ murine embryonal fibroblasts (MEFs) are a useful tool to test if the presence of lentiviral knock-down double-strand RNA in the human neuroblastoma cells distorts the results and to test whether these effects apply only to neuroinflammatory processes, or represent responses of the innate immunity system of any cell also outside the nervous system. Therefore, MEFs were subjected to FCCP treatment and the transcriptional response of the key antiviral factors was studied in the presence or absence of PINK1. As shown in Additional file 11: Fig. S4 below, FCCP treatment of MEF cells after $24 \mathrm{~h}$ led to transcriptional upregulation of murine Mavs, accompanied by downregulation of Rsad2, Ddx58, Tbk1, Irf3, Mfn1, Ifit3, and Ifit1, both in control WT cells and Pink1 $1^{-1-}$ cells. Thus, again the mitochondrial antiviral signaling factor Mavs showed a selective dependence on mitochondrial dysfunction, whereas the levels of all other antiviral key factors decreased in parallel to the FCCP-triggered autophagic elimination of mitochondria. Fibroblasts tolerate a reduction of mitochondrial mass by prominent glycolysis, whereas neuronal cells depend on mitochondria and will compensate mitophagy with mitochondrial 
biogenesis [37]. Therefore, fibroblasts and neurons may respond to FCCP with opposing regulations of mitochondria-dependent innate immunity factor levels even in control cells.

Systematic study of antiviral factor mRNAs in PINK1deficient cells after activating innate immunity with poly $(\mathrm{I}: \mathrm{C})$ as a pathogenic RNA-analogue

In view of the strong PINK1-dependent dysregulation of several sensors of pathogenic RNA and of an RNA-virus budding suppressor, namely RSAD2, IFIT3, and IFIT1 in the starvation experiment (Fig. 3c, h, i), we also stressed both cell types (neuroblastoma and MEF cells) with poly $(\mathrm{I}: \mathrm{C})$ as a pathogenic RNA-analogue and determined the transcriptional response after $16 \mathrm{~h}$ (Fig. 4a). Neuroblastoma cells responded by massive inductions of RSAD2, DDX58, IFIT3, and IFIT1, moderate induction of IRF3, and a downregulation of PINK1. The additional knockdown of PINK1 significantly blunted the upregulations of RSAD2 and DDX58 in SH-SY5Y cells. In MEF cells, again a downregulation of Pink1 was found, now paralleled by moderate downregulation of the other two mitochondrial outer membrane factors, Mfn1 and Mavs. Massive inductions of Rsad2, Ddx58, Ifit3, and Ifit1 reoccurred, now with a moderate increase of $T b k 1$. The Pink1 deletion significantly blunted the upregulations of Rsad2 and Ifit3 in MEFs.

The substantial upregulations of RNA sensors and their modulation by PINK1 encouraged us to attempt validating these effects also at the protein level, so quantitative immunoblots were performed (Fig. 4b). In neuroblastoma cells, the poly(I:C) triggered inductions were significantly blunted by PINK1 knockdown for RSAD2, DDX58, IFIT3, and IFIT1. In comparison, MEF cells also responded by strong inductions to poly(I:C), but the PINK1-dependent reductions of RSAD2, DDX58, and IFIT3 were not significant. IFIT1 could not be analyzed, since the available antibodies in our hands recognized only the human, but not the murine variant. Overall, these data clearly establish PINK1 as enhancer of anti-microbial responses and particularly of RNA sensors.

Expression analysis of key antiviral factors in primary skin fibroblasts from PARK6 patients

Particularly strong and early PINK1-dependent effects were further assessed by qPCR in unstressed primary skin fibroblasts from PARK6 patients versus control individuals, which were previously characterized regarding mitochondrial dysfunction and expression profiles and were found to constitute a useful model of PD [1, 5, 12, 19, 41-43]. In the fibroblasts of homozygous PARK6 patients, IFIT3 transcript was reduced to $31 \%(p=0.0059)$, while $R S A D 2$ transcript was increased to $307 \%(p=0.044)$ (Fig. 5a). These data in fibroblasts from manifest PARK6 patients at advanced age confirmed the previous findings in starving neuroblastoma cells, verifying that the loss of function of PINK1 leads to a deficit of the mitochondria-associated antiviral RNA sensor IFIT3, in parallel to a supersensitive induction of downstream RSAD2 (viperin) as an inhibitor of many RNA and DNA viruses. In quantitative immunoblots, IFIT3 protein was reduced to 0.27 -fold $( \pm 0.10, p=0.03$ ) (Fig. $5 \mathrm{~b})$. The RSAD2 protein levels were not significantly changed within this analysis of three patients versus three control samples. These data confirm that the IFIT3 mRNA downregulation is not offset by compensatory molecular efforts, but instead is translated into a deficit of this key antiviral factor at the protein level, thus altering innate immunity responses at the advanced age of manifest PARK6 patients.

\section{Discussion}

The progression of pathology in PD tissues and its animal and cell models is being documented intensively at the clinical, histology, imaging, neurophysiology, and molecular levels, especially in cases with monogenic pathogenesis [140-143]. There is an urgent need of risk biomarkers for the presymptomatic detection and preventive therapy, as well as of progression biomarkers for the objective quantification of disease severity and of therapeutic benefits. However, the brain tissue from patients is available only at final stages of disease, and most available autopsies are from genetically undefined and therefore heterogeneous variants of PD. Conversely, the peripheral cells and tissues from patients reflect only some initial abnormalities of pathogenesis $[19,41,43]$ and do not progress to a selective cell death. Thus, the analysis of brain tissue from postnatal age until the multimorbid old age, e.g., in PINK1-mutant or alpha-synuclein-mutant mice [30, 133, 144, 145], holds great promise. Several studies in the past have employed unbiased global OMICS approaches to screen the activity of practically all known genes [20-22, 29, 30]. Nonetheless, in spite of the enormous recent progress in PD genetics and in the characterization of corresponding disease models, it has remained difficult to identify and establish individual molecular progression biomarkers. It was expected that the levels of any such molecular marker would correlate with the severity of disease, similar to hemoglobin levels in anemia or to creatinine levels in kidney dysfunction.

Our novel progression analysis of global transcriptome profiles in PINK1-deficient brain tissue across lifespan now indicates that subsequent stages of pathology are characterized by the involvement of increasing numbers of subtly dysregulated pathways rather than stronger expression anomalies of individual candidates. This in vivo approach identified several pathways to be prominently PINK1-dependent, in good agreement with previous in vitro findings in most cases. Importantly, for the first time, we establish a temporal order and provide a quantitative value for the significance of each pathway. 


\section{a}

\section{SH-SY5Y}
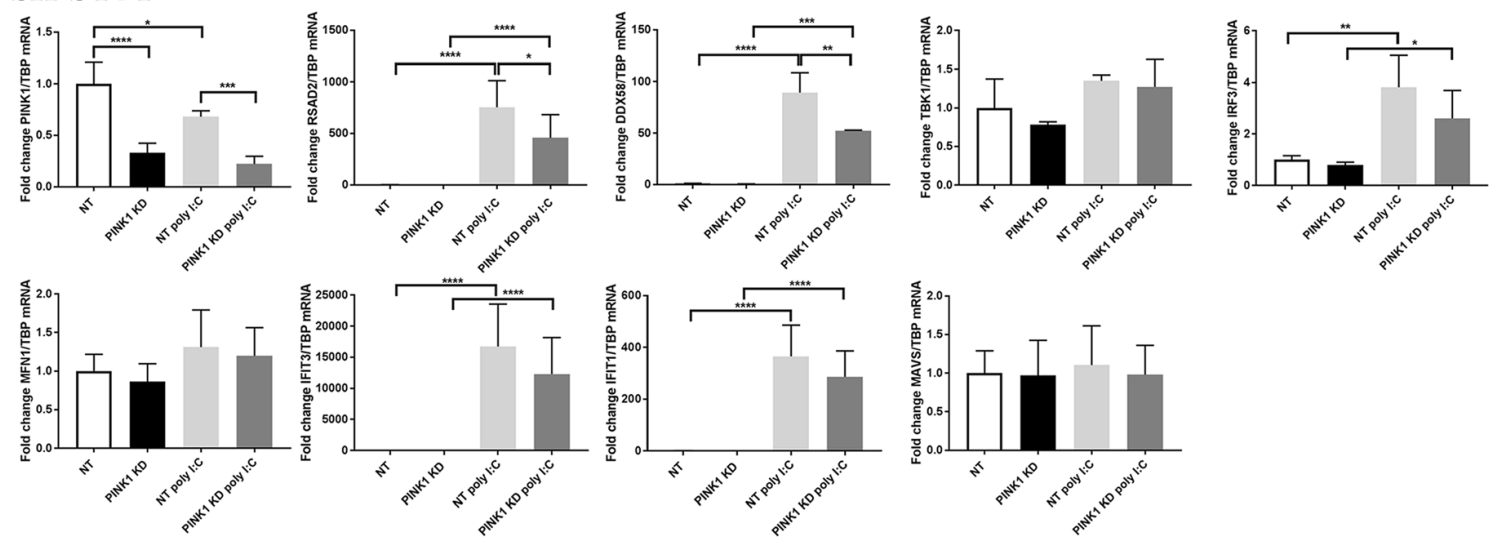

MEF
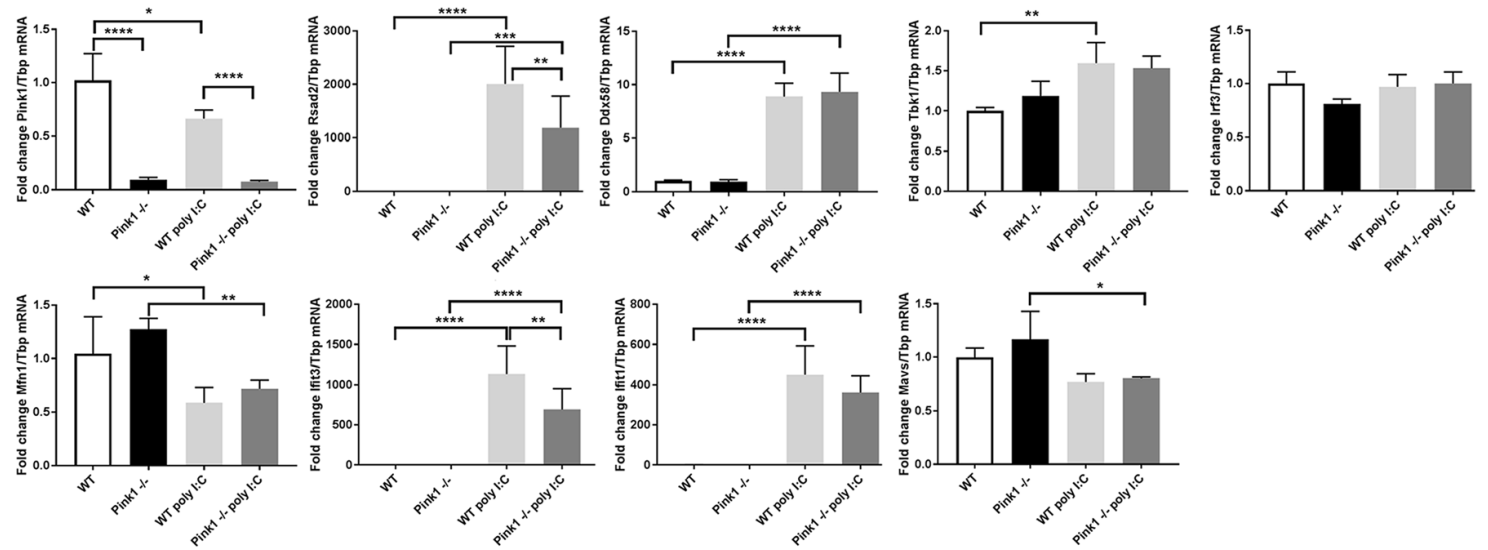

b

\section{SH-SY5Y}

RSAD2

DDX58

IFIT3

IFIT1

ACTB

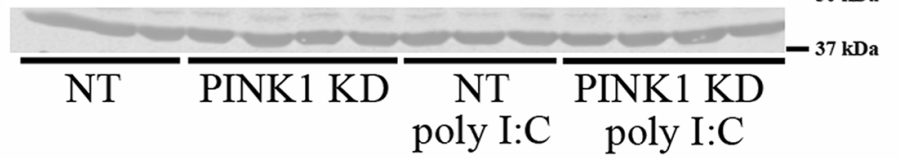

MEF

RSAD2

DDX58

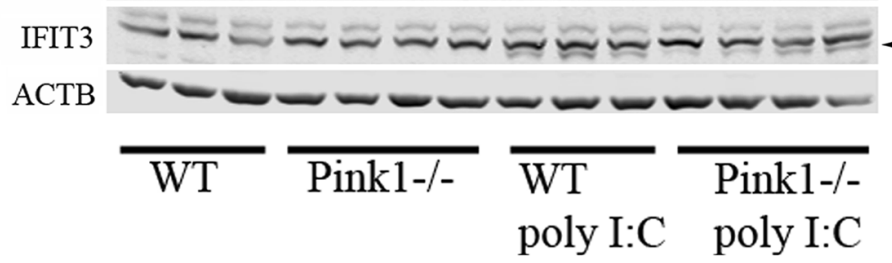

Fig. 4 (See legend on next page.)
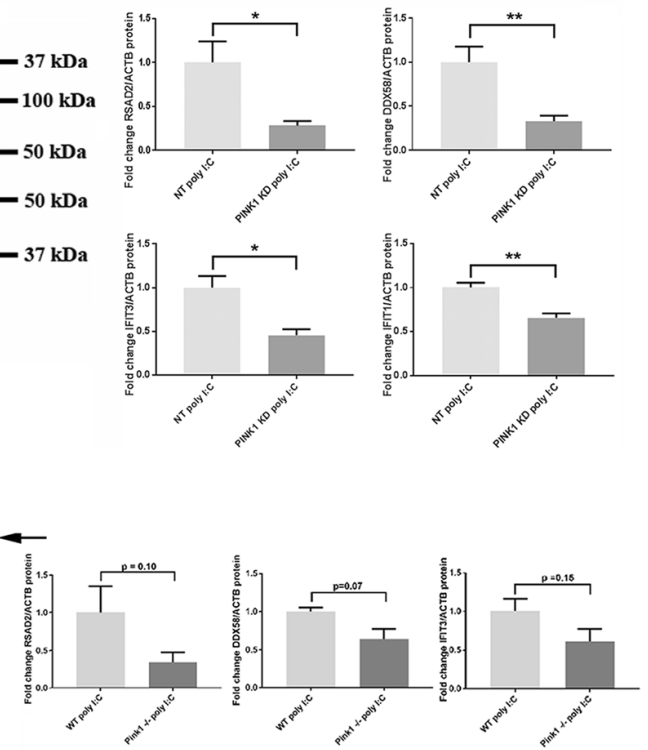
(See figure on previous page.)

Fig. 4 Poly $(I: C)$ triggered expression responses of innate immunity factors in dependence on PINK1. a Transcriptional changes: SH-SY5Y human neuroblastoma (above) and murine embryonal fibroblast cells (below) were studied regarding the expression of key inflammatory factors in untreated versus drug-treated cells, comparing control with PINK1-deficiency $~ n=8$ each for RSAD2, DDX58, IFIT3, and IFIT1 in man, and $n=4$ each for Rsad2, Ddx58, Ifit3, Ifit1 in mouse). TBP transcript levels were used as loading controls to normalize the data. The bar graphs show mean and standard error of the mean, illustrating the significance with asterisks $\left({ }^{*} p<0.05,{ }^{* *} p<0.01,{ }^{* * *} p<0.001,{ }^{* * *} p<0.0001\right)$. b Protein changes: representative quantitative immunoblots and their statistical evaluation in bar graphs ( $n=3$ WT versus 4 mutant) showed a massive induction of the RNA sensors RSAD2, DDX58, IFIT3 (in human as well as mouse), and IFIT1 (human-specific antibody), which was blunted in PINK1-deficient cells (significance demonstrable only in neural cells). The beta-Actin protein level was used as loading control to normalize the quantitative results

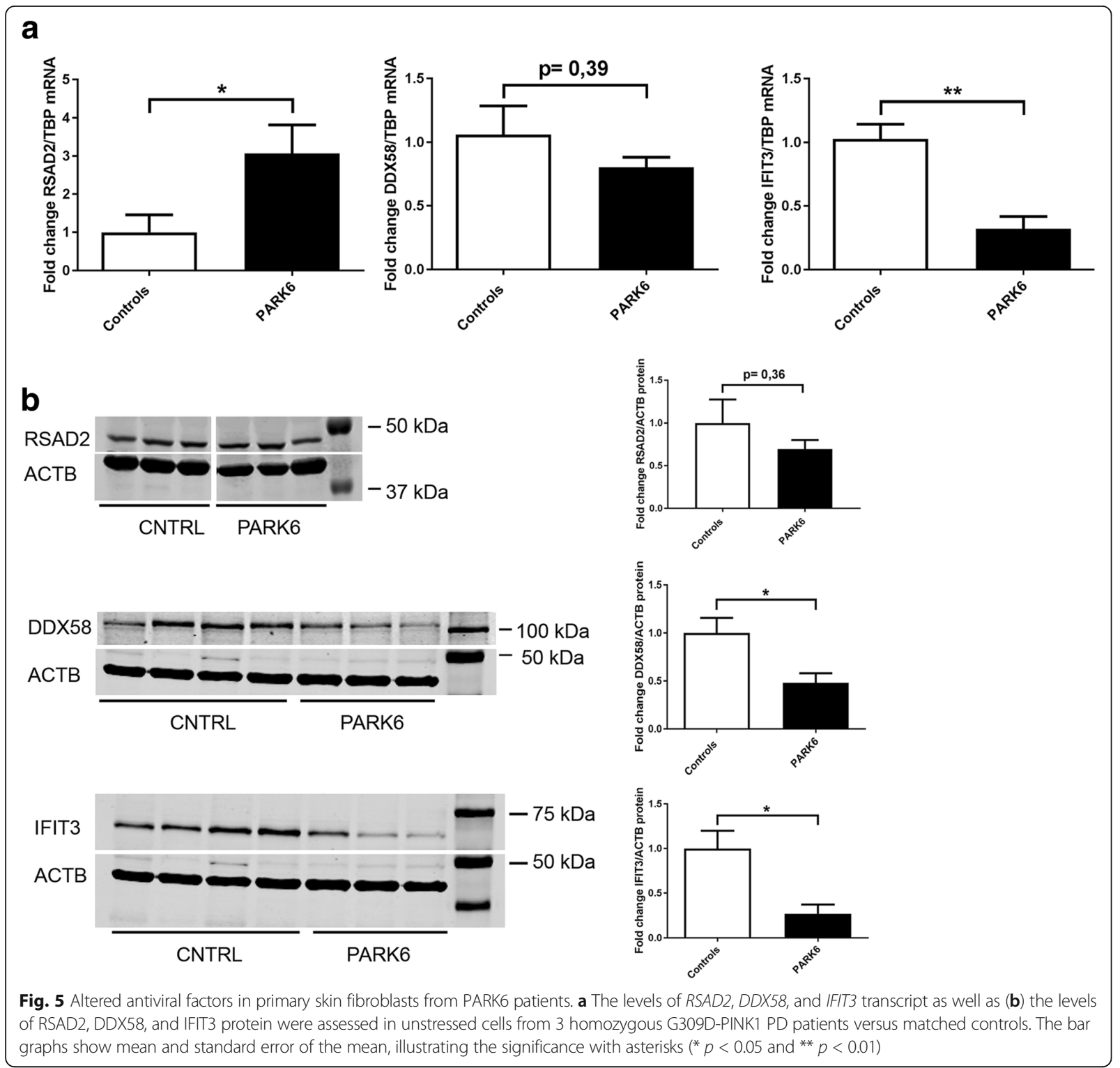


In the scenario documented, a first disease stage is defined by a mild adaptation of the nuclear splicing machinery, which persists without increase throughout all ages. This is completely novel evidence; the notion of a splicing adaptation to PINK1-deficiency is currently supported only by an OMICS study into posttranslational modifications of the brain in a genetic mouse model of PD, where a strongly altered arginine-methylation of the splicing factor PSF was observed, caused either by PINK1 deficiency or by alpha-synuclein gain of function [22]. The transcriptional dysregulation of Srsf10 as a spliceosome component was reproduced by qPCR. Given that spliceosomal alteration is a constant feature in Pink $1^{-1-}$ brain and that $U b e 3 a$ and Mapk 8 are regulated by alternative splicing, we investigated the dysregulation of their splice isoforms and demonstrated a selective effect on the shorter splice isoform of UBE3A, a ubiquitination enzyme responsible for alpha-synuclein degradation [59].

A second disease stage shows manifest anomalies in the ubiquitin-dependent degradation of proteins and in the protein processing at the endoplasmic reticulum (ER). These data are in excellent agreement with the established role of PINK1 as a ubiquitin kinase [146]. Scarce evidence exists until now on the role of PINK1 as a modifier of ER stress [147-151], but our microarray biomathematics support the notion that alterations at the ER appear earlier than mitophagy and have stronger significance. Indeed, the transcriptional dysregulation of Creb3 and Nfkbia as ER stress and inflammation factors was similarly reproduced by $\mathrm{qPCR}$ as the dysregulation of $U b e 3 a$ as a component of ubiquitination pathways. Thus, both the ubiquitin kinase effects and the early alteration of widespread protein processing support a novel concept, where PINK1 has a general role for subcellular degradation rather than a function restricted to the selective elimination of dysfunctional mitochondria. Vesicular and lysosomal pathway dysregulations are more prominent at early ages upon GSEA bioinformatics than mitochondrial pathways.

A third stage, at old age in the Pink $1^{-1-}$ mouse, cerebellar tissue shows no change of the effect size in expression dysregulation in parallel with the progression of pathology, e.g., for the components of the splicing machinery. Instead, the number of dysregulated factors and pathways, the significance of the intracellular membrane-bounded organelle enrichment, and the appearance of altered neurotransmission, mitophagy, antimicrobial, and neuroinflammatory profiles were clearly age-associated. Our experimental validation of increased Mapk8 and Nfkbia transcript levels is in agreement with a previous report about Pink1 $1^{-/-}$astrocyte JNK1 signaling [51] and with evidence for an interaction between PINK1 and the NF-kappaB pathway [152-156].
Indeed, neuroinflammation is well documented in late stages of PD [157-161]. Initially, it was thought to be triggered by the debris resulting from neuronal loss [162]. Further detailed study of genetic mouse and cell models demonstrated the neuroinflammation to precede neuronal loss [163], and current concepts propose alpha-synuclein aggregates and their extracellular extrusion to act as triggers of toll-like-receptor upregulation, cytokine release, and microglia activation [164-175]. In contrast, our observations demonstrate the enrichment of neuroinflammatory dysregulations quite early in the disease course, in brain tissues where alpha-synuclein aggregation was not detectable [29]. The concept that mitochondrial dysfunction alone is sufficient to modulate the innate immunity factors was also supported by our FCCP and poly(I:C) experiments in human neuroblastoma cells and murine embryonal fibroblasts. These data showed the mitochondrial antiviral signaling factor MAVS to be selectively responsive to proton gradient and PINK1 changes, and the induction of DDX58, IFIT3 and IFIT1 as sensors of pathogenic dsRNA and of RSAD2 as viral replication suppressor to be modified by PINK1.

Thus, we suggest that innate immunity is triggered within neurons via PINK1-associated mitochondrial dysfunction. Indeed, evidence has accumulated over the past 5 years that dysfunctional mitochondria are releasing damage-associated molecular patterns (DAMPs), e.g., the hypomethylated DNA/RNA and formylated peptides which are characteristic for bacteria and the mitochondrial endosymbiont $[176,177]$. This release triggers the innate immune system and in particular a mitochondria-associated pathway of defense against abnormal DNA/RNA [178-180]. In good compatibility with this concept, recent findings confirm that neuroinflammation in PD can be modulated by the formylpeptide receptors [181]. We have lately shown in another human hereditary disorder, Perrault syndrome, that mitochondrial dysfunction can be a strong trigger of the innate immune system via mtDNA accumulation, with subsequent early-onset infertility, growth deficits, and age-associated neurodegeneration [38]. In the pathways involved, the IFIT protein family together with DDX58 (RIG-I) is responsible of the recognition of pathogenic DNA/RNA in the cytosol, while RSAD2 (viperin) inhibits viral budding from membranes via lipid raft alteration. The mitochondria-associated MAVS/MFN1/IFIT3 complex then triggers phosphorylation and ubiquitination events that ultimately lead to NFkappaBmediated nuclear regulations and to TBK1/IRF3-mediated interferon signals for neighboring cells [111, 182] (Fig. 3a). Of course, the resulting stress metabolism and impairment of cell growth together with alterations of mitochondrial calcium buffering would influence synaptic plasticity and excitability, contributing to the known alterations of calcium 
homeostasis and neural transmission in PARK6 mouse models [44, 143, 183].

A similar mechanism could operate in PINK1 and PARKIN deficient cells, which are being used as models of PD and are known to have an impairment of selective mitophagy, as was suggested by a recent report of high visibility that PARKIN also mediates resistance to microbial invasion [18]. The PINK1dependent subtle molecular mismanagement of variably reoccurring life events such as infections (in our data mimicked by polyI:C) or hunger (mimicked by HBSS medium) may determine, if the clinical manifestation of Parkinson's disease occurs early or late in life. It was recently demonstrated that the downregulation of Ataxin-2, a lipid-storage factor and mTOR-repressor upstream from PINK1, may postpone death in a mouse model of motor neuron disease from 20 to over 300 days [135, 184-186].

The innate immunity problems of PINK1-deficient cells become detectable even at the postnatal age upon the presence of a stressor. Stress exposure of Pink $1^{-1-}$ primary neuron-rich cultures leads to the significant expression dysregulation of the abnormal DNA/RNA sensor Ifit3 and the iron-sulfur-cluster detector Rsad2, confirmed in Pink1-KO+A53T-SNCA double mutant brain, in human neuroblastoma cells, in Pink $1^{-1-}$ MEFs, and in PARK6 patient skin fibroblasts. The starvation dataset links PINK1 deficiency to a transcriptional downregulation of mitochondria-associated innate immunity factors such as MAVS, MFN1, and IFIT3, and the FCCP dataset shows altered expression of $M A V S$ and $M F N$ in MEFs and a PINK1-dependent blunting of MAVS induction in SH-SY5Y cells. MFN1 and MAVS are localized to the mitochondrial outer membrane, respond to an alteration in the mitochondrial proton gradient, and mediate the elimination of dysfunctional mitochondria via autophagy [187-190]. In addition, MAVS expression also modulates antiviral signaling via IFIT3 [112]. The starvation dataset also indicates a parallel PINK1-dependent transcriptional induction of downstream innate immunity factors such as $T B K 1, I R F 3$, and RSAD2. As shown in the poly(I:C) dataset at transcript and protein level, the regulation of IFIT3 and RSAD2 is altered by PINK1 deficiency. RSAD2 upregulation and mitochondrial relocalization triggers the lipogenesis for viral envelope formation [191, 192]. Beyond the established role of PINK1 for selective mitophagy, our novel data therefore indicate that PINK1 also modulates the mitochondria-associated anti-microbial defense pathway.

The changes in very young $\operatorname{Pink} 1^{-1-}$ brain and Pink $1^{-/-}$cells are perfectly compatible with previous in vitro observations $[84,153,154,193-198]$. Importantly, a highly visible publication was made during the final stage of our project, which reported
PINK1 and Parkin in murine macrophages and fibroblasts to repress an immune-response eliciting pathway via the trafficking of mitochondrial-derived-vesicles, but not via mitophagy [199]. The present data from Pink1 -/- mouse brain, human neuroblastoma cells, and patient fibroblasts serve as additional corroboration in vivo, providing a spatio-temporal framework and identifying crucial molecular mediators.

Still, as an important caveat, we have to mention several limitations of our findings: (I) Various technical approaches were focused on different brain regions. The previously published global transcriptome profiles had focused on midbrain/brainstem, which were selected because they are responsible for the characteristic mid-stage motor deficit of Parkinson's disease. The re-assessment of these data showed these regions to be less informative within the 2-year lifespan of mice than the cerebellum. Consequently, for a deeper understanding of the subtle early consequences of Pink1-ablation, we focused the studies of histology and ceramides on the brainstem and olfactory bulb, where Parkinson's disease starts. Furthermore, demonstration of the expected glial pathology uncovered a preferential affection of myelinated tracts. Thus, at present, it is cumbersome to extrapolate the expression data and other findings into a coherent picture of phenomenology and pathology progression in time and space, integrating observations from mouse and man, so further analyses in-depth are needed. (II) The impaired response of Pink $1^{-/}$-deficient cells against polyI:C suggests a vulnerability towards viral infections. Although we present suggestive data at the transcript, protein, and cellular level, with several artificial stressors, this issue can only unequivocally be proven once $\operatorname{Pink} 1^{-/-}$organisms are exposed to virus in future experiments, with quantification of viability and propagation rates.

\section{Conclusion}

Our present findings support the concept that neuroinflammation in PD may occur long before neuronal loss and before the formation of alpha-synuclein aggregates. PINK1-mediated dysregulations of antimicrobial defense and dysfunctional mitochondria are activating the innate immunity.

The neuroinflammatory profile appeared in Pink1deficient mouse brain after earlier ubiquitination and ER-associated degradation alterations, so these data might define markers of disease progression. Although PINK1-mutations are a rare cause of PD, PINK1 is upstream of several other Parkinson's disease genes and its inactivity results in specific mitochondrial dysfunctions as a trigger for the selective neurodegenerative 
pattern that is frequent [200-203], so our findings are probably relevant for the multifactorial sporadic old-age patients with PD.

\section{Additional files}

Additional file 1: Table S1. Global transcriptome profile of Pink $1^{-/-}$brain regions $(C=$ cerebellum, $\mathrm{M}=$ midbrain, $\mathrm{S}=$ striatum) across the mouse lifespan ( $6 \mathrm{~W}=6$ weeks, $24 \mathrm{~W}=24$ weeks $=6$ months, $18 \mathrm{M}=18$ months), showing all expression dysregulations which were consistent in all regions at all ages with significance (multiple testing adjusted $p$ value $<0.05$ ). ID identifies the Affymetrix microarray chip probe. AveExpr quantifies the average expression strength among the tissues and ages for each transcript. $F, P$ value, and AdjPvalue refer to statistical values. For each column, 3 mutant versus $3 \mathrm{WT}$ tissues were analyzed to represent a specific brain region and age. (XLS $27 \mathrm{~kb}$ )

Additional file 2: Fig. S1. Global transcriptome profile of Pink1-deficient mouse cerebellar tissue at ages 6 weeks (A), 6 months (B), and 18 months (C), illustrating the network with respect to interactions between the encoded proteins, employing the STRING web-server multiple proteins algorithm. (ZIP $11629 \mathrm{~kb})$

Additional file 3: Table S2. STRING bioinformatics assessment of all significant changes, including subtle effects. Ranked list of the significant transcript expression changes in PINK1-deficient mouse cerebellar tissue at ages 6 weeks, 6 months, and 18 months, which were used as input for the STRING analysis (A). Significant functional enrichments in the protein-proteininteraction network according to the STRING database is shown regarding GO Cellular component at 6 weeks with all factors of AdjPvalue $<0.05$ (B), 6 months with all factors of AdjPvalue $<0.05$ (C), and 18 months with all factors of AdjPvalue $<0.01$, with the term "intracellular membrane-bounded organelle" being prominent at all 3 ages (D). Prominent enrichments were highlighted in yellow color. In GO Molecular Process at 18 months with all factors of AdjPvalue $<0.01$ the relevance of stimulus- and stress-dependent pathways is apparent, e.g., endoplasmic reticulum stress, and a significant enrichment of mitophagy factors appears here in lines 495 and 518 (E). Among KEGG pathways at 18 months with all factors of AdjPvalue $<0.01$, the highest ranked pathways include "MAPK signaling", "Ubiquitin-mediated proteolysis", "Protein processing in endoplasmic reticulum" and "Bacteria invasion of epithelial cells" (F). Orange color was used to highlight some MAPK-dependent synaptic signaling pathways with significant enrichment, purple color was used for ubiquitination and protein processing pathways, green color was used for neuroinflammation pathways. (ZIP 366 kb)

Additional file 4: Table S3. STRING bioinformatics assessment with high stringency of only $>1.5$-fold downregulations with significance. Ranked KEGG pathway dysregulations are shown for cerebellum at each age. The prominent MAPK signaling is highlighted in purple color, neuroinflammation pathways in red, ubiquitination pathways in blue, endoplasmic reticulum stress response pathways in orange, the mTOR signaling pathway (mimicked by starvation stress during in vitro experiments) in dark gray, the vesicular pathways in light brown. Subsequently, the interaction diagrams are shown for each age, with components of the prominent MAPK signaling pathway highlighted in red color. Finally, a heatmap shows all components of the MAPK signaling pathway with their expression changes at each age in cerebellum, with clearly progressive downregulation for several factors at the far end. (PDF $643 \mathrm{~kb}$ )

Additional file 5: Table S4. GSEA bioinformatics assessment-ranked pathways by age. Ranked pathway dysregulations are shown separately on different datasheets for the predefined Reactome and KEGG gene sets regarding down- and upregulations, and progressive ages. Green background colors for the nominal $p$ value and the False Discovery Rate $q$ values indicate significance according to GSEA recommendations. Neuroinflammation pathways are highlighted in red color, MAPK signaling in purple, Parkinson's disease in yellow, ubiquitination pathways in blue, endoplasmic reticulum stress response pathways in orange, the mTOR signaling pathway (mimicked by starvation stress during in vitro experiments) in dark gray, the vesicular pathways in light brown. (XLSX $123 \mathrm{~kb}$ )

Additional file 6: Table S5. GSEA bioinformatics assessment-top dozen pathways with components. In Pink $1^{-1-}$ cerebellum at the age of 18 months the top dozen dysregulated KEGG pathways and the top dozen dysregulated Reactome pathways are shown with individual components. The nominal $p$ values, false discovery rate $q$ values and family wise error $p$ values are shown on top, the Rank Metric Score indicates the log2 expression values for each factor, and YES reflects significance. (PDF $1540 \mathrm{~kb}$ )

Additional file 7: Table S6. GSEA bioinformatics assessment-heat maps. Heat maps to illustrate the progression of expression dysregulation for all individual components of selected pathways of relevance, namely (A) the Reactome pathway "Negative regulators of RIG I and MDA5 signaling", (B) the Reactome pathway "Antiviral mechanism by IFN stimulated genes", (C) the Reactome pathway "Antigen processing ubiquitination proteasome degradation", and (D) the Reactome pathway "Activation of chaperone genes by Xbp1s". (PDF $135 \mathrm{~kb}$ )

Additional file 8: Fig. S2. Transcript changes of Pink1-deficient mouse cerebellar tissue at the age of 18 months in QPCR analyses represented in bar graphs. (A) Significant downregulation of Srsf10 mRNA and upregulations of Creb3 and Nfkbia mRNAs confirm the alteration within spliceosomal, ER stress and neuroinflammation pathways. The significant dysregulation of a Ube3a splice isoform is particularly interesting as a potential target of the spliceosome alterations and in view of its role in the degradation of alpha-synuclein. The scheme of Ube3a exon intron structure with the location of different Taqman assays was adapted from the Thermo Fisher Scientific internet site. (B) Significant upregulations of Mapk8 mRNA at 3 different exon junctions, together with a scheme of the Mapk 8 exon intron structure and the location of 3 different Taqman assays (modified from the Thermo Fisher Scientific internet site). Nonsignificant changes of the MAPK phosphorylation cascade components Mapk9 and Mapk14 mRNAs demonstrate the selectivity of transcriptional regulation. Significant upregulations in the downstream nuclear transcription regulators Creb3 and Nfkbia in the stress and inflammation response may reflect biological responses to the Mapk8 upregulation. The bar graphs show mean and standard error of the mean (10 Pink $1^{-/-}$versus 10 WT), illustrating the significance with asterisks $\left({ }^{*} p<0.05\right.$ and ${ }^{* *} p<0.01$ ). (TIFF $13007 \mathrm{~kb}$ )

Additional file 9: Table S7. PINK1-deficient transcriptome profile of primary neuron cultures. Ranked list of significant expression changes in global transcriptome profile of primary neuron cultures at 12 days after dissection of brains at postnatal stage, taken from 3 Pink ${ }^{-1-}$ and 3 ageand sex-matched WT controls from the same ancestors. FC = fold change. (PDF $60 \mathrm{~kb}$ )

Additional file 10: Fig. S3. Transcription dysregulation of innate immunity factors in the midbrain of 18-month-old Pink1-deficient mice with chronic stress from A53T-SNCA overexpression. The levels of the stress-triggered pro-inflammatory factor Mapk9, the lipid-droplet associated antiviral effector Rsad2 (viperin), the formylpeptide receptor activating Hebpl, and the immunostimulant Tnf (TNF-alpha) transcripts were assessed in brain from adult double mutant Pink $1^{-/-}+$A53T-SNCA mice. These data corroborate the brain tissue dysregulation of two factors that were previously found dysregulated in the global transcriptome profile of Pink $1^{-/-}$primary neuron cultures, taken from postnatal mice and maintained in vitro over 12 days. Data are displayed as bar graphs, illustrating mean values and standard error of the mean (SEM). Top transcript levels were used as loading controls to normalize the data. Significant differences were highlighted with asterisks $\left({ }^{*} p<0.05\right.$; ${ }^{* *} p<0.01$; ${ }^{* * *} p<0.001$ ). (TIFF $17632 \mathrm{~kb}$ )

Additional file 11: Fig. S4. Transcriptional response of innate immunity factors to $24 \mathrm{~h}$ treatment with uncoupling drug FCCP and subsequent mitophagy, in dependence on PINK1. Three independent experiments in SH-SY5Y human neuroblastoma (above) and murine embryonal fibroblast cells (below) documented the expression of key inflammatory factors in untreated versus drug-treated cells, comparing control with PINK1deficiency. The bar graphs show mean and standard error of the mean, illustrating the significance with asterisks $\left({ }^{*} p<0.05,{ }^{* *} p<0.01\right.$, ${ }^{* * *}$ $p<0.001$ ). (TIFF $538 \mathrm{~kb}$ )

\section{Abbreviations}

AdjPvalue: Adjusted P-value; APP: Amyloid precursor protein; AveExpr: Average expression; BSA: Bovine serum albumin; CDNA: Complementary DNA; Cer: Ceramide; DAMPs: Damage-associated molecular patterns; DNA: Deoxyribonucleic acid; dsRNA: Double-stranded RNA; ER: Endoplasmic reticulum; ES: Enrichment score; Fc epsilon RI 
signaling: Fc epsilon RI refers to the high affinity immunoglobulin E receptor; Fc gamma R-mediated phagocytosis: Fc gamma receptors phagocytose opsonized microbes; FC: Fold change; FCS: Fetal calf serum; FDR: False discovery rate; GDNF: Glial cell line-derived neurotrophic factor; GlcCer: Glucosylceramide; GO: Gene Ontology consortium; HBSS: Hank's buffered salt solution; HTLV-I: Human T-lymphotropic virus; KD: Knock-down; KEGG: Kyoto encyclopedia of genes and genomes; KO: Knock-out; LacCer: Lactosylceramide; MAPK: Mitogen-activated protein kinase mtDNA: Mitochondrial DNA; NES: Negative enrichment score; NT: Non-target knock-down; OMICS: Studies into global cell content, comprising transcriptomics, proteomics, lipidomics; PCR: Polymerase chain reaction; PD: Parkinson's disease; PINK1: PTEN-induced kinase 1; Poly(l:C): Polyinosinicpolycytidylic acid; qPCR: Quantitative reverse-transcriptase real-time PCR; RNA: Ribonucleic acid; RT: Room temperature; SEM: Standard error of the mean; SNCA: Alpha-synuclein; ssRNA: Single-strand RNA; STRING: Search tool for the retrieval of interacting genes; TBS-T: Tris-buffered saline with Tween20; TRP: Transient receptor potential; WT: Wild-type

\section{Acknowledgements}

We thank Filomena Ricciardi, Birgitt Meseck-Selchow, and Yannick Schreiber for technical assistance, Georg Auburger junior for bioinformatics help, and the animal care team at the ZFE Frankfurt (in particular the veterinarians Dr. Alf Theisen and Dr. Christa Tandi, as well as the caretakers Eleonora Daut and Birgit Janton).

\section{Funding}

Sylvia Torres-Odio was financed by the Arthur Merx Stiftung Frankfurt. The study was also supported by the DFG (GI342/3-1 to SG and CRC1039 A03 to IT), by the German Ministry of Health with the National Genome Research Network (NGFNplus, BMBF 01GS08138) and the GerontoMitoSys network (BMBF PTJ 0315584A), and by the European Union through ERAnet-RePARK (DLR 01EW1012). MM would like to thank the Luxembourg National Research Fond (FNR) for the support (FNR PEARL P16/BM/11192868 grant). The funders had no role in study design, data collection and analysis, decision to publish, or preparation of the manuscript.

\section{Availability of data and materials}

The Pink $1^{-1-}$ mouse can be commercially obtained from Jackson depository at Bar Harbor, under the code B6;129-Pink $7^{\text {tm } 1 \text { Aub } / J . ~}$

The datasets of global transcriptome data of Pink $1^{-1-}$ brain regions across lifespan, which are supporting the conclusions of this article, are available in the repository Gene Expression Omnibus, \# GSE60413, http://

www.ncbi.n/m.nih.gov/geo/query/acc.cgi?acc=GSE60414.

The stable Pink1-knock-down neuroblastoma cell line and its scramble control can be shared freely, while the PARK6 skin fibroblasts are limited in the number of aliquots and by the ethics approval for this study.

\section{Authors' contributions}

STO and JK performed starvation time course experiments, GPCR, and Westerns blots; HHH performed neuron culture and analysis; JCP and BR performed immunohistochemistry; LV contributed to lipid analyses; MW and DM performed microarray data processing; BM characterized the patients and contributed their samples; SG contributed the mouse material and supervised all lab experiments. STO, DM, IT, PNH, MM, SG, and GA conceived the project and participated in experimental design and discussion. All authors reviewed the manuscript. All authors read and approved the final manuscript.

\section{Ethics approval and consent to participate}

Animal husbandry guidelines from the German Animal Welfare Act, the Council Directive of 24 November 1986 (86/609/EWG) with Annex II and the ETS123 (European Convention for the Protection of Vertebrate Animals) were followed, keeping the lines at the FELASA-certified Central Animal Facility (ZFE) of Frankfurt University Medical School. The mice were sacrificed and analyzed under supervision of the ZFE ethics board and the Regierungspräsidium Darmstadt (K-5556)

Patient skin biopsies were performed with written informed consent and approval by the ethics commissions of the university hospitals in Granada/ Spain and Frankfurt am Main/Germany.

\section{Consent for publication}

Not applicable.

\section{Competing interests}

The authors declare that there is no competing interest regarding the publication of this manuscript.

\section{Publisher's Note}

Springer Nature remains neutral with regard to jurisdictional claims in published maps and institutional affiliations.

\section{Author details}

${ }^{1}$ Experimental Neurology, Goethe University Medical School, 60590 Frankfurt am Main, Germany. ${ }^{2}$ Institute of Clinical Pharmacology, Goethe University Medical School, 60590 Frankfurt am Main, Germany. ${ }^{3}$ Edinger-Institute (Institute of Neurology), Goethe University Medical School, 60590 Frankfurt am Main, Germany. ${ }^{4}$ Institute for Medical Genetics, Eberhard-Karls-University of Tuebingen, 72076 Tuebingen, Germany. '5epartment of Neurology, University Hospital San Cecilio, 18012 Granada, Spain. ${ }^{6}$ Max Planck Institute for Molecular Genetics, Ihnestraße 63-73, 14195 Berlin, Germany.

7 Luxembourg Centre of Neuropathology (LCNP), Luxembourg, Luxembourg. ${ }^{8}$ Department of Pathology, Laboratoire National de Santé, Dudelange, Luxembourg. ' ${ }^{2}$ uxembourg Centre for Systems Biomedicine (LCSB), University of Luxembourg, Esch-sur-Alzette, Luxembourg, Luxembourg. ${ }^{10}$ Department of Oncology, Luxembourg Institute of Health, NORLUX Neuro-Oncology Laboratory, Luxembourg, Luxembourg.

Received: 1 August 2016 Accepted: 26 July 2017

Published online: 02 August 2017

References

1. Valente EM, Abou-Sleiman PM, Caputo V, Muqit MM, Harvey K, Gispert S, Ali Z, Del Turco D, Bentivoglio AR, Healy DG, et al. Hereditary early-onset Parkinson's disease caused by mutations in PINK1. Science. 2004;304:1158-60.

2. Wauer T, Simicek M, Schubert A, Komander D. Mechanism of phospho-ubiquitininduced PARKIN activation. Nature. 2015;524:370-4

3. Lazarou M, Sliter DA, Kane LA, Sarraf SA, Wang C, Burman JL, Sideris DP, Fogel Al, Youle RJ. The ubiquitin kinase PINK1 recruits autophagy receptors to induce mitophagy. Nature. 2015;524:309-14.

4. Ordureau A, Heo JM, Duda DM, Paulo JA, Olszewski JL, Yanishevski D, Rinehart J, Schulman BA, Harper JW. Defining roles of PARKIN and ubiquitin phosphorylation by PINK1 in mitochondrial quality control using a ubiquitin replacement strategy. Proc Natl Acad Sci U S A. 2015;112:6637-42.

5. Gehrke S, Wu Z, Klinkenberg M, Sun Y, Auburger G, Guo S, Lu B. PINK1 and Parkin control localized translation of respiratory chain component mRNAs on mitochondria outer membrane. Cell Metab. 2015;21:95-108.

6. Parganlija D, Klinkenberg M, Dominguez-Bautista J, Hetzel M, Gispert S, Chimi MA, Drose S, Mai S, Brandt U, Auburger G, Jendrach M. Loss of PINK1 impairs stress-induced autophagy and cell survival. PLoS One 2014;9:e95288

7. Mai S, Klinkenberg M, Auburger G, Bereiter-Hahn J, Jendrach M. Decreased expression of Drp1 and Fis1 mediates mitochondrial elongation in senescent cells and enhances resistance to oxidative stress through PINK1. J Cell Sci. 2010;123:917-26.

8. Chen Y, Dorn GW 2nd. PINK1-phosphorylated mitofusin 2 is a Parkin receptor for culling damaged mitochondria. Science. 2013:340:471-5.

9. Scarffe LA, Stevens DA, Dawson VL, Dawson TM. Parkin and PINK1: much more than mitophagy. Trends Neurosci. 2014;37:315-24.

10. Heo JM, Ordureau A, Paulo JA, Rinehart J, Harper JW: The PINK1-PARKIN mitochondrial ubiquitylation pathway drives a program of OPTN/NDP52 recruitment and TBK1 activation to promote mitophagy. Mol Cell. 2015;60:7-20.

11. Geisler S, Holmstrom KM, Skujat D, Fiesel FC, Rothfuss OC, Kahle PJ, Springer W. PINK1/Parkin-mediated mitophagy is dependent on VDAC1 and p62/SQSTM1. Nat Cell Biol. 2010;12:119-31.

12. Exner N, Treske B, Paquet D, Holmstrom K, Schiesling C, Gispert S, CarballoCarbajal I, Berg D, Hoepken HH, Gasser T, et al. Loss-of-function of human PINK1 results in mitochondrial pathology and can be rescued by parkin. J Neurosci. 2007;27:12413-8.

13. Klinkenberg M, Gispert S, Dominguez-Bautista JA, Braun I, Auburger G, Jendrach $M$. Restriction of trophic factors and nutrients induces PARKIN expression. Neurogenetics. 2012;13:9-21. 
14. Mei Y, Zhang Y, Yamamoto K, Xie W, Mak TW, You H. FOXO3a-dependent regulation of Pink1 (Park6) mediates survival signaling in response to cytokine deprivation. Proc Natl Acad Sci U S A. 2009;106:5153-8.

15. Klein P, Muller-Rischart AK, Motori E, Schonbauer C, Schnorrer F, Winklhofer KF, Klein R. Ret rescues mitochondrial morphology and muscle degeneration of Drosophila Pink1 mutants. EMBO J. 2014;33:341-55.

16. Meka DP, Muller-Rischart AK, Nidadavolu P, Mohammadi B, Motori E, Ponna SK, Aboutalebi H, Bassal M, Annamneedi A, Finckh B, et al. Parkin cooperates with GDNF/RET signaling to prevent dopaminergic neuron degeneration. J Clin Invest. 2015;125:1873-85.

17. O'Flanagan $\mathrm{CH}, \mathrm{O}$ 'Neill C. PINK1 signalling in cancer biology. Biochim Biophys Acta. 1846;2014:590-8.

18. Manzanillo PS, Ayres JS, Watson RO, Collins AC, Souza G, Rae CS, Schneider DS, Nakamura K, Shiloh MU, Cox JS. The ubiquitin ligase parkin mediates resistance to intracellular pathogens. Nature. 2013;501:512-6.

19. Hoepken HH, Gispert S, Azizov M, Klinkenberg M, Ricciardi F, Kurz A, Morales-Gordo B, Bonin M, Riess O, Gasser T, et al. Parkinson patient fibroblasts show increased alpha-synuclein expression. Exp Neurol. 2008:212:307-13.

20. Gispert S, Brehm N, Weil J, Seidel K, Rub U, Kern B, Walter M, Roeper J, Auburger G. Potentiation of neurotoxicity in double-mutant mice with Pink1 ablation and A53T-SNCA overexpression. Hum Mol Genet. 2015;24:1061-76.

21. Auburger G, Gispert S, Jendrach M. Mitochondrial acetylation and genetic models of Parkinson's disease. Prog Mol Biol Transl Sci. 2014;127:155-82.

22. Auburger G, Gispert S, Brehm N. Methyl-arginine profile of brain from aged PINK1-KO+A53T-SNCA mice suggests altered mitochondrial biogenesis. Parkinson's Dis. 2016;2016:4686185.

23. Head E, Powell D, Gold BT, Schmitt FA. Alzheimer's disease in Down syndrome. Eur J Neurodegener Dis. 2012;1:353-64.

24. Olgiati S, Thomas A, Quadri M, Breedveld GJ, Graafland J, Eussen H, Douben $H$, de Klein A, Onofrj M, Bonifati V. Early-onset parkinsonism caused by alpha-synuclein gene triplication: Clinical and genetic findings in a novel family. Parkinsonism Relat Disord. 2015;21:981-6.

25. Konno T, Ross OA, Puschmann A, Dickson DW, Wszolek ZK. Autosomal dominant Parkinson's disease caused by SNCA duplications. Parkinsonism Relat Disord. 2016;22(Suppl 1):S1-6.

26. Chiba-Falek O, Touchman JW, Nussbaum RL. Functional analysis of intraallelic variation at NACP-Rep1 in the alpha-synuclein gene. Hum Genet. 2003;113:426-31

27. Mellick GD, Maraganore DM, Silburn PA. Australian data and meta-analysis lend support for alpha-synuclein (NACP-Rep1) as a risk factor for Parkinson's disease. Neurosci Lett. 2005;375:112-6.

28. Subramanian A, Tamayo P, Mootha VK, Mukherjee S, Ebert BL, Gillette MA Paulovich A, Pomeroy SL, Golub TR, Lander ES, Mesirov JP. Gene set enrichment analysis: a knowledge-based approach for interpreting genomewide expression profiles. Proc Natl Acad Sci U S A. 2005;102:15545-50.

29. Gispert S, Ricciardi F, Kurz A, Azizov M, Hoepken HH, Becker D, Voos W, Leuner K, Muller WE, Kudin AP, et al. Parkinson phenotype in aged PINK1deficient mice is accompanied by progressive mitochondrial dysfunction in absence of neurodegeneration. PLoS One. 2009;4:e5777.

30. Kurz A, Double KL, Lastres-Becker I, Tozzi A, Tantucci M, Bockhart V, Bonin M, Garcia-Arencibia M, Nuber S, Schlaudraff F, et al. A53T-alpha-synuclein overexpression impairs dopamine signaling and striatal synaptic plasticity in old mice. PLoS One. 2010;5:e11464.

31. Kurz A, Wohr M, Walter M, Bonin M, Auburger G, Gispert S, Schwarting RK Alpha-synuclein deficiency affects brain Foxp1 expression and ultrasonic vocalization. Neuroscience. 2010;166:785-95.

32. Franceschini A, Szklarczyk D, Frankild S, Kuhn M, Simonovic M, Roth A, Lin J, Minguez $P$, Bork $P$, von Mering $C$, Jensen LJ. STRING v9.1: protein-protein interaction networks, with increased coverage and integration. Nucleic Acids Res. 2013:41:D808-15.

33. Löffner F, Lohmann SM, Walckhoff B, Walter U, Hamprecht B. Immunocytochemical characterization of neuron-rich primary cultures of embryonic rat brain cells by established neuronal and glial markers and by monospecific antisera against cyclic nucleotide-dependent protein kinases and the synaptic vesicle protein synapsin I. Brain Res. 1986;363:205-21.

34. Schiffmann S, Sandner J, Birod K, Wobst I, Angioni C, Ruckhaberle E, Kaufmann M, Ackermann H, Lotsch J, Schmidt H, et al. Ceramide synthases and ceramide levels are increased in breast cancer tissue. Carcinogenesis. 2009;30:745-52.
35. Zschiebsch K, Fischer C, Pickert G, Haussler A, Radeke H, Grosch S, Ferreiros N, Geisslinger G, Werner ER, Tegeder I. Tetrahydrobiopterin attenuates DSSevoked colitis in mice by rebalancing redox and lipid signalling. J Crohn's Colitis. 2016;10:965-78.

36. Schmittgen TD, Livak KJ. Analyzing real-time PCR data by the comparative C(T) method. Nat Protoc. 2008:3:1101-8.

37. Rakovic A, Shurkewitsch K, Seibler P, Grunewald A, Zanon A, Hagenah J, Krainc D, Klein C. Phosphatase and tensin homolog (PTEN)-induced putative kinase 1 (PINK1)-dependent ubiquitination of endogenous Parkin attenuates mitophagy: study in human primary fibroblasts and induced pluripotent stem cell-derived neurons. J Biol Chem. 2013;288:2223-37.

38. Gispert S, Parganlija D, Klinkenberg M, Drose S, Wittig I, Mittelbronn M, Grzmil P, Koob S, Hamann A, Walter M, et al. Loss of mitochondrial peptidase Clpp leads to infertility, hearing loss plus growth retardation via accumulation of CLPX, mtDNA and inflammatory factors. Hum Mol Genet. 2013:22:4871-87.

39. Liu Y, Zhang YB, Liu TK, Gui JF. Lineage-specific expansion of IFIT gene family: an insight into coevolution with IFN gene family. PLoS One. 2013:8:e66859.

40. Ueta M, Kawai T, Yokoi N, Akira S, Kinoshita S. Contribution of IPS-1 to polyl: C-induced cytokine production in conjunctival epithelial cells. Biochem Biophys Res Commun. 2011;404:419-23.

41. Hoepken HH, Gispert S, Morales B, Wingerter O, Del Turco D, Mulsch A, Nussbaum RL, Muller K, Drose S, Brandt U, et al. Mitochondrial dysfunction, peroxidation damage and changes in glutathione metabolism in PARK6. Neurobiol Dis. 2007;25:401-11.

42. Klinkenberg M, Thurow N, Gispert S, Ricciardi F, Eich F, Prehn JH, Auburger G, Kogel D. Enhanced vulnerability of PARK6 patient skin fibroblasts to apoptosis induced by proteasomal stress. Neuroscience. 2010;166:422-34.

43. Auburger G, Klinkenberg M, Drost J, Marcus K, Morales-Gordo B, Kunz WS, Brandt U, Broccoli V, Reichmann H, Gispert S, Jendrach M. Primary skin fibroblasts as a model of Parkinson's disease. Mol Neurobiol. 2012;46:20-7.

44. Dehorter N, Lozovaya N, Mdzomba BJ, Michel FJ, Lopez C, Tsintsadze V, Tsintsadze T, Klinkenberg M, Gispert S, Auburger G, Hammond C. Subthalamic lesion or levodopa treatment rescues giant GABAergic currents of PINK1-deficient striatum. J Neurosci. 2012;32:18047-53.

45. Shkreta L, Toutant J, Durand M, Manley JL, Chabot B. SRSF10 connects DNA damage to the alternative splicing of transcripts encoding apoptosis, cellcycle control, and DNA repair factors. Cell Rep. 2016;17:1990-2003.

46. Wu S, Majeed SR, Evans TM, Camus MD, Wong NM, Schollmeier Y, Park M, Muppidi JR, Reboldi A, Parham P, et al. Clathrin light chains' role in selective endocytosis influences antibody isotype switching. Proc Natl Acad Sci U S A. 2016;113:9816-21.

47. Cheng C, Sharp PA. Regulation of CD44 alternative splicing by SRm160 and its potential role in tumor cell invasion. Mol Cell Biol. 2006;26:362-70.

48. Fukuda A, Nakadai T, Shimada M, Hisatake K. Heterogeneous nuclear ribonucleoprotein $\mathrm{R}$ enhances transcription from the naturally configured c-fos promoter in vitro. J Biol Chem. 2009;284:23472-80.

49. Reches A, Nachmani D, Berhani O, Duev-Cohen A, Shreibman D, Ophir Y, Seliger B, Mandelboim O. HNRNPR regulates the expression of classical and nonclassical MHC class I proteins. J Immunol. 2016;196:4967-76.

50. Kim KS, Kim JS, Park JY, Suh YH, Jou I, Joe EH, Park SM. DJ-1 associates with lipid rafts by palmitoylation and regulates lipid rafts-dependent endocytosis in astrocytes. Hum Mol Genet. 2013;22:4805-17.

51. Choi I, Kim J, Jeong HK, Kim B, Jou I, Park SM, Chen L, Kang UJ, Zhuang X Joe EH. PINK1 deficiency attenuates astrocyte proliferation through mitochondrial dysfunction, reduced AKT and increased p38 MAPK activation, and downregulation of EGFR. Glia. 2013;61:800-12.

52. Park JH, Ko J, Park YS, Park J, Hwang J, Koh HC. Clearance of damaged mitochondria through PINK1 stabilization by JNK and ERK MAPK signaling in chlorpyrifos-treated neuroblastoma cells. Mol Neurobiol. 2016;54:1844-57.

53. Kitada T, Pisani A, Porter DR, Yamaguchi H, Tscherter A, Martella G, Bonsi P, Zhang C, Pothos EN, Shen J. Impaired dopamine release and synaptic plasticity in the striatum of PINK1-deficient mice. Proc Natl Acad Sci U S A. 2007:104:11441-6.

54. Martella G, Platania P, Vita D, Sciamanna G, Cuomo D, Tassone A, Tscherter A, Kitada T, Bonsi P, Shen J, Pisani A. Enhanced sensitivity to group II mGlu receptor activation at corticostriatal synapses in mice lacking the familial parkinsonism-linked genes PINK1 or Parkin. Exp Neurol. 2009;215:388-96.

55. Madeo G, Schirinzi T, Martella G, Latagliata EC, Puglisi F, Shen J, Valente EM, Federici M, Mercuri NB, Puglisi-Allegra S, et al. PINK1 heterozygous 
mutations induce subtle alterations in dopamine-dependent synaptic plasticity. Mov Disord. 2014;29:41-53.

56. Feligioni M, Mango D, Piccinin S, Imbriani P, lannuzzi F, Caruso A, De Angelis F Blandini F, Mercuri NB, Pisani A, Nistico R. Subtle alterations of excitatory transmission are linked to presynaptic changes in the hippocampus of PINK1deficient mice. Synapse. 2016;70:223-30.

57. Mishra A, Godavarthi SK, Maheshwari M, Goswami A, Jana NR. The ubiquitin ligase E6-AP is induced and recruited to aggresomes in response to proteasome inhibition and may be involved in the ubiquitination of Hsp70bound misfolded proteins. J Biol Chem. 2009;284:10537-45.

58. LaVoie MJ, Cortese GP, Ostaszewski BL, Schlossmacher MG. The effects of oxidative stress on parkin and other E3 ligases. J Neurochem. 2007;103:2354-68.

59. Mulherkar SA, Sharma J, Jana NR. The ubiquitin ligase E6-AP promotes degradation of alpha-synuclein. J Neurochem. 2009;110:1955-64.

60. Latour S, Aguilar C. XIAP deficiency syndrome in humans. Semin Cell Dev Biol. 2015;39:115-23.

61. Sandebring A, Thomas KJ, Beilina A, van der Brug M, Cleland MM, Ahmad R, Miller DW, Zambrano I, Cowburn RF, Behbahani H, et al. Mitochondrial alterations in PINK1 deficient cells are influenced by calcineurin-dependent dephosphorylation of dynamin-related protein 1. PLoS One. 2009:4:e5701.

62. Su J, Yin J, Qin W, Sha S, Xu J, Jiang C. Role for pro-inflammatory cytokines in regulating expression of GABA transporter type 1 and 3 in specific brain regions of kainic acid-induced status epilepticus. Neurochem Res. 2015;40:621-7.

63. Lu K, Psakhye I, Jentsch S. Autophagic clearance of polyQ proteins mediated by ubiquitin-Atg 8 adaptors of the conserved CUET protein family. Cell. 2014;158:549-63.

64. Hirota Y, Yamashita S, Kurihara Y, Jin X, Aihara M, Saigusa T, Kang D, Kanki T. Mitophagy is primarily due to alternative autophagy and requires the MAPK1 and MAPK14 signaling pathways. Autophagy. 2015;11:332-43.

65. Park JH, Ko J, Park YS, Park J, Hwang J, Koh HC. Clearance of damaged mitochondria through PINK1 stabilization by JNK and ERK MAPK signaling in chlorpyrifos-treated neuroblastoma cells. Mol Neurobiol. 2017:54:1844-57.

66. Cang X, Wang X, Liu P, Wu X, Yan J, Chen J, Wu G, Jin Y, Xu F, Su J, Wan C. PINK1 alleviates palmitate induced insulin resistance in HepG2 cells by suppressing ROS mediated MAPK pathways. Biochem Biophys Res Commun. 2016;478:431-8.

67. Yamamoto K, Furukawa MT, Fukumura K, Kawamura A, Yamada T, Suzuki H,

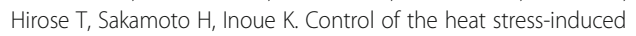
alternative splicing of a subset of genes by hnRNP K. Genes Cells. 2016:21:1006-14.

68. DenBoer LM, Hardy-Smith PW, Hogan MR, Cockram GP, Audas TE, Lu R. Luman is capable of binding and activating transcription from the unfolded protein response element. Biochem Biophys Res Commun. 2005;331:113-9.

69. Liang G, Audas TE, Li Y, Cockram GP, Dean JD, Martyn AC, Kokame K, Lu R. Luman/CREB3 induces transcription of the endoplasmic reticulum (ER) stress response protein Herp through an ER stress response element. Mol Cell Biol. 2006:26:7999-8010.

70. Jang SW, Kim YS, Lee YH, Ko J. Role of human LZIP in differential activation of the NF-kappaB pathway that is induced by CCR1-dependent chemokines. J Cell Physiol. 2007;211:630-7.

71. Jang SW, Kim YS, Kim YR, Sung HJ, Ko J. Regulation of human LZIP expression by NF-kappaB and its involvement in monocyte cell migration induced by Lkn-1. J Biol Chem. 2007;282:11092-100.

72. Li X, Long J, He T, Belshaw R, Scott J. Integrated genomic approaches identify major pathways and upstream regulators in late onset Alzheimer's disease. Sci Rep. 2015;5:12393.

73. Ahmad A, Crupi R, Campolo M, Genovese T, Esposito E, Cuzzocrea S. Absence of TLR4 reduces neurovascular unit and secondary inflammatory process after traumatic brain injury in mice. PLoS One. 2013;8:e57208.

74. Perier C, Bove J, Wu DC, Dehay B, Choi DK, Jackson-Lewis V, Rathke-Hartlieb S, Bouillet P, Strasser A, Schulz JB, et al. Two molecular pathways initiate mitochondria-dependent dopaminergic neurodegeneration in experimental Parkinson's disease. Proc Natl Acad Sci U S A. 2007:104:8161-6.

75. Ren H, Fu K, Mu C, Li B, Wang D, Wang G. DJ-1, a cancer and Parkinson's disease associated protein, regulates autophagy through JNK pathway in cancer cells. Cancer Lett. 2010;297:101-8.

76. Dzamko N, Zhou J, Huang Y, Halliday GM. Parkinson's disease-implicated kinases in the brain; insights into disease pathogenesis. Front Mo Neurosci. 2014;7:57.
77. Brown M, Strudwick N, Suwara M, Sutcliffe LK, Mihai AD, Ali AA, Watson JN, Schroder M. An initial phase of JNK activation inhibits cell death early in the endoplasmic reticulum stress response. J Cell Sci. 2016; 129:2317-28.

78. Han SY, Kim SH, Heasley LE. Differential gene regulation by specific gain-offunction JNK1 proteins expressed in Swiss 3T3 fibroblasts. J Biol Chem. 2002; 277:47167-74.

79. Dreskin SC, Thomas GW, Dale SN, Heasley LE. Isoforms of Jun kinase are differentially expressed and activated in human monocyte/macrophage (THP-1) cells. J Immunol. 2001;166:5646-53.

80. Sentelle RD, Senkal CE, Jiang W, Ponnusamy S, Gencer S, Selvam SP, Ramshesh VK, Peterson YK, Lemasters JJ, Szulc ZM, et al. Ceramide targets autophagosomes to mitochondria and induces lethal mitophagy. Nat Chem Biol. 2012;8:831-8.

81. Mayo L, Trauger SA, Blain M, Nadeau M, Patel B, Alvarez JI, Mascanfroni ID, Yeste A, Kivisakk P, Kallas K, et al. Regulation of astrocyte activation by glycolipids drives chronic CNS inflammation. Nat Med. 2014;20:1147-56.

82. Ferrazza R, Cogo S, Melrose H, Bubacco L, Greggio E, Guella G, Civiero L, Plotegher N. LRRK2 deficiency impacts ceramide metabolism in brain. Biochem Biophys Res Commun. 2016;478:1141-6.

83. Wang G, Dinkins M, He Q, Zhu G, Poirier C, Campbell A, Mayer-Proschel M Bieberich E. Astrocytes secrete exosomes enriched with proapoptotic ceramide and prostate apoptosis response 4 (PAR-4): potential mechanism of apoptosis induction in Alzheimer disease (AD). J Biol Chem. 2012;287:21384-95.

84. Kim J, Byun JW, Choi I, Kim B, Jeong HK, Jou I, Joe E. PINK1 deficiency enhances inflammatory cytokine release from acutely prepared brain slices. Exp Neurobiol. 2013;22:38-44.

85. Bolon B, Dorman DC, Bonnefoi MS, Randall HW, Morgan KT. Histopathologic approaches to chemical toxicity using primary cultures of dissociated neural cells grown in chamber slides. Toxicol Pathol. 1993;21:465-79.

86. Ahlemeyer B, Kolker S, Zhu Y, Hoffmann GF, Krieglstein J. Cytosine arabinofuranoside-induced activation of astrocytes increases the susceptibility of neurons to glutamate due to the release of soluble factors. Neurochem Int. 2003;42:567-81.

87. Meier ID, Bernreuther C, Tilling T, Neidhardt J, Wong YW, Schulze C, Streichert T, Schachner M. Short DNA sequences inserted for gene targeting can accidentally interfere with off-target gene expression. FASEB J. 2010;24:1714-24.

88. Meijer $\mathrm{AH}$, van der Vaart M. DRAM1 promotes the targeting of mycobacteria to selective autophagy. Autophagy. 2014;10:2389-91.

89. Tatura R, Kraus T, Giese A, Arzberger T, Buchholz M, Hoglinger G, Muller U. Parkinson's disease: SNCA-, PARK2-, and LRRK2- targeting microRNAs elevated in cingulate gyrus. Parkinsonism Relat Disord. 2016;33:115-21.

90. Zhao Y, Haney MJ, Gupta R, Bohnsack JP, He Z, Kabanov AV, Batrakova EV. GDNF-transfected macrophages produce potent neuroprotective effects in Parkinson's disease mouse model. PLoS One. 2014;9:e106867.

91. Lynch C, Tristem M. A co-opted gypsy-type LTR-retrotransposon is conserved in the genomes of humans, sheep, mice, and rats. Curr Biol. 2003;13:1518-23.

92. Syapin PJ. Regulation of haeme oxygenase-1 for treatment of neuroinflammation and brain disorders. Br J Pharmacol. 2008:155:623-40.

93. Koliaraki V, Kollias G. A new role for myeloid $\mathrm{HO}-1$ in the innate to adaptive crosstalk and immune homeostasis. Adv Exp Med Biol. 2011;780:101-11.

94. Devosse T, Dutoit R, Migeotte I, De Nadai P, Imbault V, Communi D, Salmon I, Parmentier M. Processing of HEBP1 by cathepsin D gives rise to F2L, the agonist of formyl peptide receptor 3. J Immunol. 2011:187:1475-85.

95. Le Blanc I, Luyet PP, Pons V, Ferguson C, Emans N, Petiot A, Mayran N, Demaurex N, Faure J, Sadoul R, et al. Endosome-to-cytosol transport of viral nucleocapsids. Nat Cell Biol. 2005;7:653-64.

96. Li L, Cheng FW, Wang F, Jia B, Luo X, Zhang SQ. The activation of TLR7 regulates the expression of VEGF, TIMP1, MMP2, IL-6, and IL-15 in Hela cells. Mol Cell Biochem. 2014;389:43-9.

97. Shi M, Movius J, Dator R, Aro P, Zhao Y, Pan C, Lin X, Bammler TK, Stewart T, Zabetian CP, et al. Cerebrospinal fluid peptides as potential Parkinson disease biomarkers: a staged pipeline for discovery and validation. Mol Cell Proteomics. 2015:14:544-55.

98. Phalke S, Nickel O, Walluscheck D, Hortig F, Onorati MC, Reuter G. Retrotransposon silencing and telomere integrity in somatic cells of Drosophila depends on the cytosine-5 methyltransferase DNMT2. Nat Genet. 2009;41:696-702 
99. Durdevic Z, Hanna K, Gold B, Pollex T, Cherry S, Lyko F, Schaefer M. Efficient RNA virus control in Drosophila requires the RNA methyltransferase Dnmt2. EMBO Rep. 2013;14:269-75.

100. Vivekanandan P, Daniel HD, Kannangai R, Martinez-Murillo F, Torbenson M. Hepatitis $B$ virus replication induces methylation of both host and viral DNA. J Virol. 2010;84:4321-9.

101. Thiagarajan D, Dev RR, Khosla S. The DNA methyltranferase Dnmt2 participates in RNA processing during cellular stress. Epigenetics. 2011;6:103-13.

102. Mattijssen S, Pruijn GJ. Viperin, a key player in the antiviral response. Microbes Infect/Institut Pasteur. 2012;14:419-26.

103. Goossens KE, Karpala AJ, Rohringer A, Ward A, Bean AG. Characterisation of chicken viperin. Mol Immunol. 2015;63:373-80.

104. Helbig KJ, Beard MR. The role of viperin in the innate antiviral response. J Mol Biol. 2014;426:1210-9.

105. Saitoh T, Satoh T, Yamamoto N, Uematsu S, Takeuchi O, Kawai T, Akira S. Antiviral protein Viperin promotes Toll-like receptor 7- and Toll-like receptor 9-mediated type I interferon production in plasmacytoid dendritic cells. Immunity. 2011;34:352-63.

106. Qiu LQ, Cresswell P, Chin KC. Viperin is required for optimal Th2 responses and T-cell receptor-mediated activation of NF-kappaB and AP-1. Blood. 2009;113:3520-9.

107. Yatabe T, Mochizuki S, Takizawa M, Chijiiwa M, Okada A, Kimura T, Fujita Y, Matsumoto $\mathrm{H}$, Toyama Y, Okada Y. Hyaluronan inhibits expression of ADAMTS4 (aggrecanase-1) in human osteoarthritic chondrocytes. Ann Rheum Dis. 2009;68:1051-8.

108. Phillips KL, Jordan-Mahy N, Nicklin MJ, Le Maitre CL. Interleukin-1 receptor antagonist deficient mice provide insights into pathogenesis of human intervertebral disc degeneration. Ann Rheum Dis. 2013;72:1860-7.

109. Satoh K, Suzuki N, Yokota H. ADAMTS-4 (a disintegrin and metalloproteinase with thrombospondin motifs) is transcriptionally induced in beta-amyloid treated rat astrocytes. Neurosci Lett. 2000;289:177-80.

110. Boussadia $O$, Niepmann $M$, Creancier L, Prats AC, Dautry F, JacqueminSablon $\mathrm{H}$. Unr is required in vivo for efficient initiation of translation from the internal ribosome entry sites of both rhinovirus and poliovirus. J Virol. 2003;77:3353-9.

111. Zhou X, Michal JJ, Zhang L, Ding B, Lunney JK, Liu B, Jiang Z. Interferon induced IFIT family genes in host antiviral defense. Int J Biol Sci. 2013;9:200-8.

112. Liu XY, Chen W, Wei B, Shan YF, Wang C. IFN-induced TPR protein IFIT3 potentiates antiviral signaling by bridging MAVS and TBK1. J Immunol. 2011;187:2559-68.

113. Bai L, Merchant JL. ZBP-89 promotes growth arrest through stabilization of p53. Mol Cell Biol. 2001;21:4670-83.

114. Sayin VI, Nilton A, Ibrahim MX, Agren $P$, Larsson E, Petit MM, Hulten LM, Stahlman M, Johansson BR, Bergo MO, Lindahl P. Zfp148 deficiency causes lung maturation defects and lethality in newborn mice that are rescued by deletion of p53 or antioxidant treatment. PLoS One. 2013:8:e55720.

115. Nigg EA. Cyclin-dependent kinase 7: at the cross-roads of transcription, DNA repair and cell cycle control? Curr Opin Cell Biol. 1996:8:312-7.

116. Ko LJ, Shieh SY, Chen X, Jayaraman L, Tamai K, Taya Y, Prives C, Pan ZQ. p53 is phosphorylated by CDK7-cyclin $\mathrm{H}$ in a p36MAT1-dependent manner. Mol Cell Biol. 1997;17:7220-9.

117. Nilson KA, Guo J, Turek ME, Brogie JE, Delaney E, Luse DS, Price DH. THZ1 reveals roles for $\mathrm{Cdk7}$ in co-transcriptional capping and pausing. Mol Cell. 2015;59:576-87.

118. Aulas A, Stabile S, Vande Velde C. Endogenous TDP-43, but not FUS, contributes to stress granule assembly via G3BP. Mol Neurodegener 2012;7:54

119. Ash PE, Vanderweyde TE, Youmans KL, Apicco DJ, Wolozin B. Pathological stress granules in Alzheimer's disease. Brain Res. 2014;1584:52-8.

120. Heck MV, Azizov M, Stehning T, Walter M, Kedersha N, Auburger G. Dysregulated expression of lipid storage and membrane dynamics factors in Tia1 knockout mouse nervous tissue. Neurogenetics. 2014;15:135-44.

121. Waterfield M, Khan IS, Cortez JT, Fan U, Metzger T, Greer A, Fasano K, Martinez-Llordella M, Pollack JL, Erle DJ, et al. The transcriptional regulator Aire coopts the repressive ATF7ip-MBD1 complex for the induction of immunotolerance. Nat Immunol. 2014;15:258-65.

122. Liu Q, Liu L, Zhao Y, Zhang J, Wang D, Chen J, He Y, Wu J, Zhang Z, Liu Z. Hypoxia induces genomic DNA demethylation through the activation of HIF-1alpha and transcriptional upregulation of MAT2A in hepatoma cells. Mol Cancer Ther. 2011;10:1113-23.
123. Liu Q, Chen J, Liu L, Zhang J, Wang D, Ma L, He Y, Liu Y, Liu Z, Wu J. The X protein of hepatitis $B$ virus inhibits apoptosis in hepatoma cells through enhancing the methionine adenosyltransferase $2 \mathrm{~A}$ gene expression and reducing S-adenosylmethionine production. J Biol Chem. 2011;286:17168-80.

124. Blanco MG, Matos J. Hold your horSSEs: controlling structure-selective endonucleases MUS81 and Yen1/GEN1. Front Genet. 2015;6:253.

125. Dehe PM, Coulon S, Scaglione S, Shanahan P, Takedachi A, Wohlschlegel JA, Yates JR 3rd, Llorente B, Russell P, Gaillard PH. Regulation of Mus81-Eme1 Holliday junction resolvase in response to DNA damage. Nat Struct Mol Biol. 2013:20:598-603.

126. Hofmann TG, Glas C, Bitomsky N. HIPK2: A tumour suppressor that controls DNA damage-induced cell fate and cytokinesis. BioEssays. 2013;35:55-64.

127. Nardinocchi L, Puca R, Givol D, D'Orazi G. HIPK2-a therapeutical target to be (re)activated for tumor suppression: role in p53 activation and HIF-1alpha inhibition. Cell Cycle. 2010;9:1270-5.

128. Findlay VJ, Tapiero H, Townsend DM. Sulfiredoxin: a potential therapeutic agent? Biomed Pharmacother. 2005;59:374-9.

129. Roychoudhury J, Clark JP, Gracia-Maldonado G, Unnisa Z, Wunderlich M, Link KA, Dasgupta N, Aronow B, Huang G, Mulloy JC, Kumar AR. MEIS1 regulates an HLF-oxidative stress axis in MLL-fusion gene leukemia. Blood. 2015;125:2544-52.

130. Otto T, Fandrey J. Thyroid hormone induces hypoxia-inducible factor 1alpha gene expression through thyroid hormone receptor beta/retinoid $x$ receptor alpha-dependent activation of hepatic leukemia factor. Endocrinology. 2008;149:2241-50.

131. Kleven MD, Dlakic M, Lawrence CM. Characterization of a single b-type heme, FAD, and metal binding sites in the transmembrane domain of sixtransmembrane epithelial antigen of the prostate (STEAP) family proteins. J Biol Chem. 2015;290:22558-69.

132. Hagele S, Behnam B, Borter E, Wolfe J, Paasch U, Lukashev D, Sitkovsky M, Wenger RH, Katschinski DM. TSGA10 prevents nuclear localization of the hypoxia-inducible factor (HIF)-1alpha. FEBS Lett. 2006;580:3731-8.

133. Gispert S, Kurz A, Brehm N, Rau K, Walter M, Riess O, Auburger G. Complexin-1 and Foxp1 expression changes are novel brain effects of alpha-synuclein pathology. Mol Neurobiol. 2015;52:57-63.

134. Schwarting RK, Sedelis M, Hofele K, Auburger GW, Huston JP. Straindependent recovery of open-field behavior and striatal dopamine deficiency in the mouse MPTP model of Parkinson's disease. Neurotox Res. 1999;1:41-56.

135. Sen NE, Drost J, Gispert $S$, Torres-Odio S, Damrath E, Klinkenberg M, Hamzeiy H, Akdal G, Gulluoglu H, Basak AN, Auburger G. Search for SCA2 blood RNA biomarkers highlights Ataxin-2 as strong modifier of the mitochondrial factor PINK1 levels. Neurobiol Dis. 2016;96:115-26.

136. Sen NE, Gispert S, Auburger G. PINK1 and Ataxin-2 as modifiers of growth. Oncotarget. 2017:8:32382-3.

137. Auburger $G$, Sen NE, Meierhofer D, Basak AN, Gitler AD. Efficient prevention of neurodegenerative diseases by depletion of starvation response factor Ataxin-2. Trends Neurosci. 2017:40:507-16.

138. Becker LA, Huang B, Bieri G, Ma R, Knowles DA, Jafar-Nejad P, Messing J, Kim HJ, Soriano A, Auburger G, et al. Therapeutic reduction of ataxin2 extends lifespan and reduces pathology in TDP-43 mice. Nature. 2017;544:367-71.

139. Azkona G, Lopez de Maturana R, Del Rio P, Sousa A, Vazquez N, Zubiarrain A Jimenez-Blasco D, Bolanos JP, Morales B, Auburger G, et al. LRRK2 expression is deregulated in fibroblasts and neurons from Parkinson patients with mutations in PINK1. Mol Neurobiol. 2016. doi:10.1007/s12035-016-0303-7.

140. Hernandez DG, Paisan-Ruiz C, McInerney-Leo A, Jain S, Meyer-Lindenberg A, Evans EW, Berman KF, Johnson J, Auburger G, Schaffer AA, et al. Clinical and positron emission tomography of Parkinson's disease caused by LRRK2. Ann Neurol. 2005;57:453-6.

141. Braak H, Bohl JR, Muller CM, Rub U, de Vos RA, Del Tredici K. Stanley Fahn Lecture 2005: the staging procedure for the inclusion body pathology associated with sporadic Parkinson's disease reconsidered. Mov Dis. 2006;21:2042-51.

142. Tuin I, Voss U, Kessler K, Krakow K, Hilker R, Morales B, Steinmetz H, Auburger G. Sleep quality in a family with hereditary parkinsonism (PARK6). Sleep Med. 2008;9:684-8.

143. Kessler KR, Hamscho N, Morales B, Menzel C, Barrero F, Vives F, Gispert S, Auburger $\mathrm{G}$. Dopaminergic function in a family with the PARK6 form of autosomal recessive Parkinson's syndrome. J Neural Transm. 2005;112:1345-53. 
144. Platt NJ, Gispert S, Auburger G, Cragg SJ. Striatal dopamine transmission is subtly modified in human A53Talpha-synuclein overexpressing mice. PLoS One. 2012;7:e36397.

145. Subramaniam M, Althof D, Gispert S, Schwenk J, Auburger G, Kulik A, Fakler B, Roeper J. Mutant alpha-synuclein enhances firing frequencies in dopamine substantia nigra neurons by oxidative impairment of A-type potassium channels. J Neurosci. 2014;34:13586-99.

146. Durcan TM, Fon EA. The three 'P's of mitophagy: PARKIN, PINK1, and posttranslational modifications. Genes Dev. 2015:29:989-99.

147. Samann J, Hegermann J, von Gromoff E, Eimer S, Baumeister R, Schmidt E. Caenorhabditits elegans LRK-1 and PINK-1 act antagonistically in stress response and neurite outgrowth. J Biol Chem. 2009;284:16482-91.

148. Li L, Hu GK. Pink1 protects cortical neurons from thapsigargin-induced oxidative stress and neuronal apoptosis. Biosci Rep. 2015;35:e00174.

149. Erpapazoglou $Z$, Corti $O$. The endoplasmic reticulum/mitochondria interface: a subcellular platform for the orchestration of the functions of the PINK1Parkin pathway? Biochem Soc Trans. 2015;43:297-301.

150. Van Laar VS, Roy N, Liu A, Rajprohat S, Arnold B, Dukes AA, Holbein CD, Berman SB. Glutamate excitotoxicity in neurons triggers mitochondria and endoplasmic reticulum accumulation of Parkin, and in the presence of $\mathrm{N}$-acetyl cysteine, mitophagy. Neurobiol Dis. 2015;74:180-93.

151. Celardo I, Costa AC, Lehmann S, Jones C, Wood N, Mencacci NE, Mallucci GR, Loh SH, Martins LM. Mitofusin-mediated ER stress triggers neurodegeneration in pink1/parkin models of Parkinson's disease. Cell Death Dis. 2016:7:e2271

152. Sha D, Chin LS, Li L. Phosphorylation of parkin by Parkinson disease-linked kinase PINK1 activates parkin E3 ligase function and NF-kappaB signaling. Hum Mol Genet. 2010;19:352-63.

153. Akundi RS, Huang Z, Eason J, Pandya JD, Zhi L, Cass WA, Sullivan PG, Bueler H. Increased mitochondrial calcium sensitivity and abnormal expression of innate immunity genes precede dopaminergic defects in Pink1-deficient mice. PLoS One. 2011:6:e16038.

154. Lee HJ, Jang SH, Kim H, Yoon JH, Chung KC. PINK1 stimulates interleukin1 beta-mediated inflammatory signaling via the positive regulation of TRAF6 and TAK1. Cell Mol Life Sci. 2012;69:3301-15.

155. Duan X, Tong J, Xu Q, Wu Y, Cai F, Li T, Song W. Upregulation of human PINK1 gene expression by NFkappaB signalling. Mol Brain. 2014;7:57.

156. Lim GG, Chua DS, Basil AH, Chan HY, Chai C, Arumugam T, Lim KL. Cytosolic PTEN-induced Putative Kinase 1 Is Stabilized by the NF-kappaB Pathway and Promotes Non-selective Mitophagy. J Biol Chem. 2015;290:16882-93.

157. Nolan YM, Sullivan AM, Toulouse A. Parkinson's disease in the nuclear age of neuroinflammation. Trends Mol Med. 2013:19:187-96.

158. More SV, Kumar H, Kim IS, Song SY, Choi DK. Cellular and molecular mediators of neuroinflammation in the pathogenesis of Parkinson's disease. Mediat Inflamm. 2013;2013:952375.

159. Hunot S, Hirsch EC. Neuroinflammatory processes in Parkinson's disease. Ann Neurol. 2003:53(Suppl 3):S49-58. discussion S58-60

160. Ouchi Y, Yagi S, Yokokura M, Sakamoto M. Neuroinflammation in the living brain of Parkinson's disease. Parkinsonism Relat Disord. 2009; 15(Suppl 3):S200-4.

161. Dzamko N, Geczy CL, Halliday GM. Inflammation is genetically implicated in Parkinson's disease. Neuroscience. 2015;302:89-102.

162. Mosley RL, Benner EJ, Kadiu I, Thomas M, Boska MD, Hasan K, Laurie C, Gendelman HE. Neuroinflammation, oxidative stress and the pathogenesis of Parkinson's disease. Clin Neurosci Res. 2006:6:261-81.

163. Sekiyama K, Sugama S, Fujita M, Sekigawa A, Takamatsu Y, Waragai M, Takenouchi T, Hashimoto M. Neuroinflammation in Parkinson's disease and related disorders: a lesson from genetically manipulated mouse models of al pha-synucleinopathies. Parkinson's Dis. 2012;2012:271732.

164. Sanchez-Guajardo V Tentillier N, Romero-Ramos M. The relation between alpha-synuclein and microglia in Parkinson's disease: recent developments. Neuroscience. 2015;302:47-58.

165. Moehle MS, West AB. M1 and M2 immune activation in Parkinson's disease: foe and ally? Neuroscience. 2015;302:59-73.

166. Hirsch EC, Jenner P, Przedborski S. Pathogenesis of Parkinson's disease. Mov Dis. 2013:28:24-30

167. Lema Tome CM, Tyson T, Rey NL, Grathwohl S, Britschgi M, Brundin P. Inflammation and alpha-synuclein's prion-like behavior in Parkinson's disease-is there a link? Mol Neurobiol. 2013;47:561-74.
168. Beraud D, Twomey M, Bloom B, Mittereder A, Ton V, Neitzke K, Chasovskikh S, Mhyre TR, Maguire-Zeiss KA. Alpha-synuclein alters toll-like receptor expression. Front Neurosci. 2011:5:80.

169. Lastres-Becker I, Ulusoy A, Innamorato NG, Sahin G, Rabano A, Kirik D, Cuadrado A. Alpha-synuclein expression and Nrf2 deficiency cooperate to aggravate protein aggregation, neuronal death and inflammation in earlystage Parkinson's disease. Hum Mol Genet. 2012;21:3173-92.

170. Gao HM, Kotzbauer PT, Uryu K, Leight S, Trojanowski JQ, Lee VM. Neuroinflammation and oxidation/nitration of alpha-synuclein linked to dopaminergic neurodegeneration. J Neurosci. 2008;28:7687-98.

171. Theodore S, Cao S, McLean PJ, Standaert DG. Targeted overexpression of human alpha-synuclein triggers microglial activation and an adaptive immune response in a mouse model of Parkinson disease. J Neuropathol Exp Neurol. 2008:67:1149-58.

172. Kim C, Ho DH, Suk JE, You S, Michael S, Kang J, Joong Lee S, Masliah E, Hwang D, Lee HJ, Lee SJ. Neuron-released oligomeric alpha-synuclein is an endogenous agonist of TLR2 for paracrine activation of microglia. Nat Commun. 2013;4:1562.

173. Reynolds AD, Glanzer JG, Kadiu I, Ricardo-Dukelow M, Chaudhuri A, Ciborowski P, Cerny R, Gelman B, Thomas MP, Mosley RL, Gendelman HE. Nitrated alpha-synuclein-activated microglial profiling for Parkinson's disease. J Neurochem. 2008;104:1504-25.

174. Tang $Y$, Le W. Differential roles of $M 1$ and $M 2$ microglia in neurodegenerative diseases. Mol Neurobiol. 2016;53:1181-94.

175. Schapansky J, Nardozzi JD, LaVoie MJ. The complex relationships between microglia, alpha-synuclein, and LRRK2 in Parkinson's disease. Neuroscience. 2015;302:74-88.

176. Zhang Q, Raoof M, Chen Y, Sumi Y, Sursal T, Junger W, Brohi K, Itagaki K, Hauser CJ. Circulating mitochondrial DAMPs cause inflammatory responses to injury. Nature. 2010;464:104-7.

177. Manfredi AA, Rovere-Querini P. The mitochondrion-a Trojan horse that kicks off inflammation? N Engl J Med. 2010:362:2132-4.

178. West AP, Khoury-Hanold W, Staron M, Tal MC, Pineda CM, Lang SM, Bestwick M, Duguay BA, Raimundo N, MacDuff DA, et al. Mitochondrial DNA stress primes the antiviral innate immune response. Nature. 2015;520:553-7.

179. Arnoult D, Soares F, Tattoli I, Girardin SE. Mitochondria in innate immunity. EMBO Rep. 2011;12:901-10.

180. Scott I. The role of mitochondria in the mammalian antiviral defense system. Mitochondrion. 2010;10:316-20.

181. Mollica A, Stefanucci A, Costante R, Pinnen F. Role of formyl peptide receptors (FPR) in abnormal inflammation responses involved in neurodegenerative diseases. Anti-inflamm Anti-allergy Agents Med Chem. 2012;11:20-36

182. Diamond MS, Farzan M. The broad-spectrum antiviral functions of IFIT and IFITM proteins. Nat Rev Immunol. 2013;13:46-57.

183. Carron R, Filipchuk A, Nardou R, Singh A, Michel FJ, Humphries MD, Hammond C. Early hypersynchrony in juvenile PINK1 $(-) /(-)$ motor cortex is rescued by antidromic stimulation. Front Syst Neurosci. 2014;8:95.

184. Becker LA, Huang B, Bieri G, Ma R, Knowles DA, Jafar-Nejad P, Messing J, Kim HJ, Soriano A, Auburger G, et al. Therapeutic reduction of ataxin 2 extends lifespan and reduces pathology in TDP-43 mice. Nature. 2017;544: $367-71$.

185. Lastres-Becker I, Nonis D, Eich F, Klinkenberg M, Gorospe M, Kotter P, Klein FA, Kedersha N, Auburger G. Mammalian ataxin-2 modulates translation control at the pre-initiation complex via PI3K/mTOR and is induced by starvation. Biochim Biophys Acta. 1862;2016:1558-69.

186. Bar DZ, Charar C, Dorfman J, Yadid T, Tafforeau L, Lafontaine DL, Gruenbaum Y. Cell size and fat content of dietary-restricted Caenorhabditis elegans are regulated by ATX-2, an mTOR repressor. Proc Natl Acad Sci U S A. 2016:113:E4620-9.

187. Sun X, Sun L, Zhao Y, Li Y, Lin W, Chen D, Sun Q. MAVS maintains mitochondrial homeostasis via autophagy. Cell Discov. 2016;2:16024.

188. Ziviani E, Tao RN, Whitworth AJ. Drosophila parkin requires PINK1 for mitochondrial translocation and ubiquitinates mitofusin. Proc Natl Acad Sci U S A. 2010;107:5018-23.

189. Gegg ME, Cooper JM, Chau KY, Rojo M, Schapira AH, Taanman JW. Mitofusin 1 and mitofusin 2 are ubiquitinated in a PINK1/parkin-dependent manner upon induction of mitophagy. Hum Mol Genet. 2010;19:4861-70.

190. Koshiba T, Yasukawa K, Yanagi Y, Kawabata S. Mitochondrial membrane potential is required for MAVS-mediated antiviral signaling. Sci Signal. 2011;4:ra7. 
191. Seo JY, Cresswell P. Viperin regulates cellular lipid metabolism during human cytomegalovirus infection. PLoS Pathog. 2013;9:e1003497.

192. Seo JY, Yaneva R, Hinson ER, Cresswell P. Human cytomegalovirus directly induces the antiviral protein viperin to enhance infectivity. Science. 2011;332:1093-7.

193. Ellis Gl, Zhi L, Akundi R, Bueler H, Marti F. Mitochondrial and cytosolic roles of PINK1 shape induced regulatory T-cell development and function. Eur J Immunol. 2013;43:3355-60.

194. Gurung P, Lukens JR, Kanneganti TD. Mitochondria: diversity in the regulation of the NLRP3 inflammasome. Trends Mol Med. 2015;21:193-201.

195. Lee HJ, Chung KC. PINK1 positively regulates IL-1 beta-mediated signaling through Tollip and IRAK1 modulation. J Neuroinflammation. 2012:9·271.

196. Yunfu W, Guangjian L, Ping Z, Yanpeng S, Xiaoxia F, Wei H, Jiang Y, Jingquan $\mathrm{H}$, Songlin W, Hongyan Z, et al. PINK1 and its familial Parkinson's disease-associated mutation regulate brain vascular endothelial inflammation. J Mol Neurosci. 2014;53:109-16.

197. Kang R, Zeng L, Xie Y, Yan Z, Zhou B, Cao L, Klionsky DJ, Tracey KJ, Li J, Wang $\mathrm{H}$, et al. A novel PINK1- and PARK2-dependent protective neuroimmune pathway in lethal sepsis. Autophagy. 2016;12:2374-85.

198. Zhu J, Qu Y, Lin Z, Zhao F, Zhang L, Huang Y, Jiang C, Mu D. Loss of PINK1 inhibits apoptosis by upregulating alpha-synuclein in inflammationsensitized hypoxic-ischemic injury in the immature brains. Brain Res 1653;2016:14-22.

199. Matheoud D, Sugiura A, Bellemare-Pelletier A, Laplante A, Rondeau C, Chemali M, Fazel A, Bergeron JJ, Trudeau LE, Burelle Y, et al. Parkinson's disease-related proteins PINK1 and Parkin repress mitochondrial antigen presentation. Cell. 2016;166:314-27.

200. Ryan BJ, Hoek S, Fon EA, Wade-Martins R. Mitochondrial dysfunction and mitophagy in Parkinson's: from familial to sporadic disease. Trends Biochem Sci. 2015:40:200-10

201. Celardo I, Martins LM, Gandhi S. Unravelling mitochondrial pathways to Parkinson's disease. Br J Pharmacol. 2014:171:1943-57.

202. de Vries RL, Przedborski S. Mitophagy and Parkinson's disease: be eaten to stay healthy. Mol Cell Neurosci. 2013;55:37-43.

203. Corti O, Lesage S, Brice A. What genetics tells us about the causes and mechanisms of Parkinson's disease. Physiol Rev. 2011;91:1161-218.

\section{Submit your next manuscript to BioMed Central and we will help you at every step:}

- We accept pre-submission inquiries

- Our selector tool helps you to find the most relevant journal

- We provide round the clock customer support

- Convenient online submission

- Thorough peer review

- Inclusion in PubMed and all major indexing services

- Maximum visibility for your research 


\subsection{Publication II}

\section{Declaration of author contributions}

Title: Systematic Surveys of Iron Homeostasis Mechanisms Reveal Ferritin Superfamily and Nucleotide Surveillance Regulation to be Modified by PINK1 Absence

Status: published

Journal: Cells (MDPI), 2020, doi: 10.3390/cells9102229

Contributing authors: Key J (JK), Sen NE (NES), Arsović A (AA), Krämer S (SK), Hülse R (RH), Khan NN (NNK), Meierhofer D (DM), Gispert S (SG), Koepf G (GK), Auburger G (GA)

What are the contributions of the doctoral candidate and her co-authors?

\section{(1) Concept and design}

Doctoral candidate (JK): $65 \%$

Co-author DM: $5 \%$

Co-author GA: $\mathbf{3 0 \%}$

\section{(2) Conducting tests and experiments}

Doctoral candidate (JK): $\mathbf{9 0 \%}$ (expression analyses, biochemical characterization, cell culture experiments, immunostaining)

Co-author DM: 10\% (mass spectrometry proteomics)

\section{(3) Compilation of data sets and figures}

Doctoral candidate (JK): $\mathbf{8 0 \%}$ (proteome data, expression analyses)

Co-author DM: $\mathbf{1 0 \%}$ (proteome data)

Co-author GA: 10\% (proteome data)

\section{(4) Analysis and interpretation of data}

Doctoral candidate (JK): 75\% (expression analyses, proteome analyses)

Co-author DM: $\mathbf{5 \%}$ (mass spectrometry proteomics)

Co-author GA: $\mathbf{2 0 \%}$ (interpretation of all data)

\section{(5) Drafting of manuscript}

Doctoral candidate (JK): $65 \%$

Co-author DM: $\mathbf{5 \%}$

Co-author GA: $\mathbf{3 0 \%}$

Date and place

Signature of the doctoral candidate

Date and place

Signature of the supervisor 
Article

\title{
Systematic Surveys of Iron Homeostasis Mechanisms Reveal Ferritin Superfamily and Nucleotide Surveillance Regulation to be Modified by PINK1 Absence
}

\author{
Jana Key ${ }^{1,2}$, Nesli Ece Sen ${ }^{1,2}$, Aleksandar Arsović ${ }^{1}$, Stella Krämer ${ }^{1}$, Robert Hülse ${ }^{1}$, \\ Natasha Nadeem Khan ${ }^{1}$, David Meierhofer ${ }^{3}{ }^{\infty}$, Suzana Gispert ${ }^{1}$, Gabriele Koepf ${ }^{1}$ and \\ Georg Auburger 1,* \\ 1 Experimental Neurology, Medical School, Goethe University, 60590 Frankfurt am Main, Germany; \\ key@stud.uni-frankfurt.de (J.K.); nesliecesen@gmail.com (N.E.S.); arsovicalexandar@gmail.com (A.A.); \\ stella.kraemer@yahoo.de (S.K.); robert.huelse@gmail.com (R.H.); natzkhan.203@gmail.com (N.N.K.); \\ Gispert-Sanchez@em.uni-frankfurt.de (S.G.); Gabriele.Koepf@kgu.de (G.K.) \\ 2 Faculty of Biosciences, Goethe-University, Altenhöferallee 1, 60438 Frankfurt am Main, Germany \\ 3 Max Planck Institute for Molecular Genetics, Ihnestraße 63-73, 14195 Berlin, Germany; \\ Meierhof@molgen.mpg.de \\ * Correspondence: auburger@em.uni-frankfurt.de; Tel.: +49-(0)-69-6301-7428
}

Received: 3 September 2020; Accepted: 29 September 2020; Published: 2 October 2020

\begin{abstract}
Iron deprivation activates mitophagy and extends lifespan in nematodes. In patients suffering from Parkinson's disease (PD), PINK1-PRKN mutations via deficient mitophagy trigger iron accumulation and reduce lifespan. To evaluate molecular effects of iron chelator drugs as a potential PD therapy, we assessed fibroblasts by global proteome profiles and targeted transcript analyses. In mouse cells, iron shortage decreased protein abundance for iron-binding nucleotide metabolism enzymes (prominently XDH and ferritin homolog RRM2). It also decreased the expression of factors with a role for nucleotide surveillance, which associate with iron-sulfur-clusters (ISC), and are important for growth and survival. This widespread effect included prominently Nthl1-Ppat-Bdh2, but also mitochondrial Glrx5-Nfu1-Bola1, cytosolic Aco1-Abce1-Tyw5, and nuclear Dna2-Elp3-Pold1-Prim2. Incidentally, upregulated Pink1-Prkn levels explained mitophagy induction, the downregulated expression of Slc25a28 suggested it to function in iron export. The impact of PINK1 mutations in mouse and patient cells was pronounced only after iron overload, causing hyperreactive expression of ribosomal surveillance factor $A b c e 1$ and of ferritin, despite ferritin translation being repressed by IRP1. This misregulation might be explained by the deficiency of the ISC-biogenesis factor GLRX5. Our systematic survey suggests mitochondrial ISC-biogenesis and post-transcriptional iron regulation to be important in the decision, whether organisms undergo PD pathogenesis or healthy aging.
\end{abstract}

Keywords: synuclein; CPT1A; MMP14; PYGL; Tfrc; Ireb2; Pgrmc1; Hmox1; Cyp46a1; Slc11a2; Slc25a37; iron overload versus deprivation; nucleotide metabolism; neurodegeneration

\section{Introduction}

Intracellular iron metabolism in mammalian cells is crucial for their proper functions. Free iron molecules can be toxic to cells, leading to the production of ROS (reactive oxygen species) and lipid peroxidation terminating in a programmed cell death known as ferroptosis [1], so there have to be strict regulatory mechanisms. Iron metabolism affects the whole cell, since iron is taken up from extracellular space either via transferrin or in a transferrin-independent manner, then reduced for 
detoxification and distributed within cells, for example to mitochondria, where iron is utilized for iron-sulfur-cluster (ISC) production and heme generation [2-4]. The correct function of dozens of proteins in mitochondria, cytosol, and nucleus depends on the insertion of ISC $[5,6]$, so the regulation of iron homeostasis is crucial. ISC are unstable during oxidative stress periods, but play important roles in a wide range of cellular reactions, e.g., electron transfer, catalysis of enzymatic reactions, regulation of gene expression (e.g., via association with IRP1/IRP2 (iron regulatory proteins) to exert post-transcriptional control over ferritin and transferrin receptor levels), and in the quality control of nucleotides, thus controlling genome integrity [7-9]. Similarly, heme is needed as a cofactor of cytochrome proteins within mitochondria, but also of cytosolic cytochrome P450 proteins, globins, iron-regulatory proteins, peroxidases, catalase, and specific ion-channels [10].

Recently, it was shown in the nematode Caenorhabditis elegans that an extrinsic factor, namely the availability of iron, has a strong impact on lifespan. The suppression of iron uptake by a chelator drug, as well as the silencing of Fxn (FRATAXIN) (cursive lowercase letters refer to the DNA/RNA level in rodents, while uppercase letters refer to the protein) as a mitochondrial ISC biogenesis factor, both extended the lifespan via mitochondrial stress and activation of PINK1/PARKIN-dependent mitophagy. Downstream effects of this pathological scenario included the elevated expression of globins, which bind to iron in the form of heme [11]. It was also reported that natural inducers of mitophagy, such as urolithin A, can extend the lifespan in C. elegans [12]. We were intrigued by these observations since a converse situation is observed in man: Defective mitophagy due to Pink1/Prkn mutations shortens the lifespan and leads to the accumulation of iron during a neurodegenerative process that we know as Parkinson's disease (PD) [13-15].

The serine-threonine kinase PINK1 (PTEN induced kinase 1) associates with the outer mitochondrial membrane and phosphorylates cytosolic proteins to coordinate the PARKIN-dependent autophagic degradation of damaged or aged mitochondria, in a process known as mitophagy [16-18]. PINK1 and PRKN (encoding PARKIN) get transcriptionally induced in human neuroblastoma cells after serum deprivation or nutrient starvation [19], linking dietary restriction to mitophagy. Mutations in PINK1 and PRKN lead to autosomal recessive juvenile-onset variants of PD, which were named PARK6 and PARK2, respectively [14,20]. Iron distribution is altered in the brains of all PD patients [21,22], with a preferential increase of iron levels in the midbrain substantia nigra $[23,24]$, where the loss of dopaminergic neurons is observed. These findings add to the established concept that iron accumulation contributes to neurodegenerative processes. In dopaminergic midbrain neurons, much of the stored iron is absorbed onto neuromelanin granules, while other neurons and brain glial cells can only deposit it as ferritin protein complexes [25]. It remains unclear to what degree in diverse cells the pathological redistribution of excess iron occurs towards the labile iron pool (LIP), to mitochondria, or to ferritin with its ferroxidase site, where ferrous iron ( +2 oxidation state) can be converted to ferric iron (+3 oxidation state) and thus stored [26]. It is conceivable that altered turnover of iron-containing proteins contributes to the iron toxicity in PD. One piece of evidence was found in a neurotoxic PD model via 5-day acute exposure to the respiratory complex-I inhibitor 1-methyl-4-phenyl-1,2,3,6-tetrahydropyridine (MPTP), where an acute increase of ferritin light-chain and mitochondrial ferritin (Ftmt) was reported together with a protective function of mitochondrial ferritin overexpression [27].

Detailed studies of PD pathogenesis confirmed that (i) PINK1 mutations trigger iron accumulation in the midbrain of patients [28], (ii) Pink1 deficiency-mediated iron accumulation may involve degradation of mitochondrial membrane iron transporters SLC25A37 (Mfrn1) and SLC25A28 (Mfrn2) (solute carrier family 25, member 37/28) [29,30], (iii) iron chelation-induced mitophagy can be observed in PARK2 patient primary fibroblasts [31], (iv) Pink1-dependent phenotypes in flies can be suppressed by mitochondrial aconitase (ACO2), while superoxide-dependent inactivation of the ACO2 [4Fe-4S] cluster triggers iron toxicity that is reversed by mitochondrial ferritin overexpression [32]. Thus, there is a close link between the mitochondrial dysfunction underlying PD on the one hand, with the homeostasis of iron, ISC, and heme on the other hand. It is important now to elucidate the relevant molecular events 
of iron homeostasis and how it is modulated in PD, to define molecular biomarkers of PD progression, and to understand how extrinsic factors may modify the disease course.

In the chronic state of mutant brain tissue, it is very hard to detect these anomalies. Mitophagy is relevant for only a few among hundreds or thousands of mitochondria per cell at any given time, and the accumulation of iron is an insidious process over decades in PD patient brain, so the compensatory efforts needed are minimal. Therefore, global expression profiles of Pink1 $^{-1-}$ mouse brain showed only subtle evidence of deficient mitophagy and altered mitochondrial biogenesis [20,33-35], the dysregulated expression of heme-related transcripts Hmox1 (Heme oxygenase 1) and Hebp1 (Heme binding protein 1) was noted only upon culture of mouse Pink $1^{-/-}$primary cortical neurons [35], and limited survival of the Pink1 $1^{--}$mouse was observed only after additional overexpression of toxic alpha-synuclein (SNCA) [20,36]. In general, PINK1- and PARKIN-deficient mice show signs of altered mitophagy and neurodegeneration only in the presence of further stressors such as mitochondrial mutations, exhaustive exercise, or bacterial infections [37-40]. A lifespan effect was also not detectable in C. elegans Pink1- and Parkin-mutants. In contrast, the survival of Drosophila melanogaster flies with depletion of PINK1 or PARKIN was significantly shortened by degeneration of wing muscles, due to the massive exercise and energetic demand during flight [20,41-43].

Not only altered mitophagy but also autophagy and mitochondrial dysfunction in general, have strong effects on iron homeostasis and lifespan, as was demonstrated in C. elegans for the so-called mit-mutants, where dysfunctions of the electron transfer chain trigger unexpected longevity [44-46]. In another well-established model of rapid aging, the fungus Podospora anserina, a simple depletion of the mitochondrial matrix protease $\mathrm{ClpP}$ (Caseinolytic mitochondrial matrix peptidase proteolytic subunit) results in a prolonged lifespan [47]. Again, mice with $C l p P$ deletion were reported to have altered survival with higher resistance to metabolic stress and bacterial infections [48,49], as well as iron and hemoglobin accumulation (https://www.mousephenotype.org/data/genes/MGI:1858213). In contrast to Pink1 mutant cells [17,50], stable ClpP mutants showed no evidence of oxidative stress [51], enhancing the doubts whether ROS have a central process in the control of lifespan [52,53] and emphasizing the notion that iron levels may be more relevant stressors than ROS.

Although our studies now were done in vitro with peripheral cells using massive acute iron stress and are thus limited to extreme situations never observed in vivo, we believe that similar subtle long term effects can occur in the nervous system and might contribute to neurodegenerative diseases.

\section{Results}

\subsection{Global Proteome Profile Adaptations upon Iron Overload and Iron Depletion in WT and Pink1 $1^{-/-}$Mouse Embryonic Fibroblast (MEF) Cells}

The initial quality evaluation of stressed WT MEF (wildtype mouse embryonic fibroblasts) cultures showed that iron overload mediated by ferric ammonium citrate (FAC) exposure triggered relatively few abundance changes, in comparison to iron shortage mediated by 2,2'-Bipyridine (22BP) administration, and Pink1-ablation also caused few effects (Figure 1B and Supplementary Table S1), upon mass spectrometry and subsequent statistical analysis. Venn diagram analyses were employed to identify factors that were dysregulated in more than one condition (Supplementary Table S1).

Normal culture media contain iron concentrations that were maximized to stimulate cell growth, so iron depletion is expected to have the highest impact. Further plausibility assessments showed that the heavy and light ferritin chain subunits (FTH1 and FTL1) were in direct correlation to iron availability; also the heme oxygenase (HMOX1) increased after FAC treatment (Figure 1C), confirming well-known regulations. A strong induction of ferritin protein by FAC administration over $24 \mathrm{~h}$, for example, was previously documented in rat primary neurons by a mass spectrometry profiling study [54]. Our additional tests by RT-qPCR also for less abundant factors demonstrated the known indirect correlation of iron availability with the transferrin receptor (encoded by the gene Tfrc) and aconitase-3 (Ireb2) levels, as well as its direct correlation with the divalent metal transporter 1 (Slc11a2, 
Solute carrier family 11 member 2) mRNA levels (Figure 1D). Taken together, the culture settings used validated previous knowledge and provided a useful approach for systematic explorations into yet unknown effects.

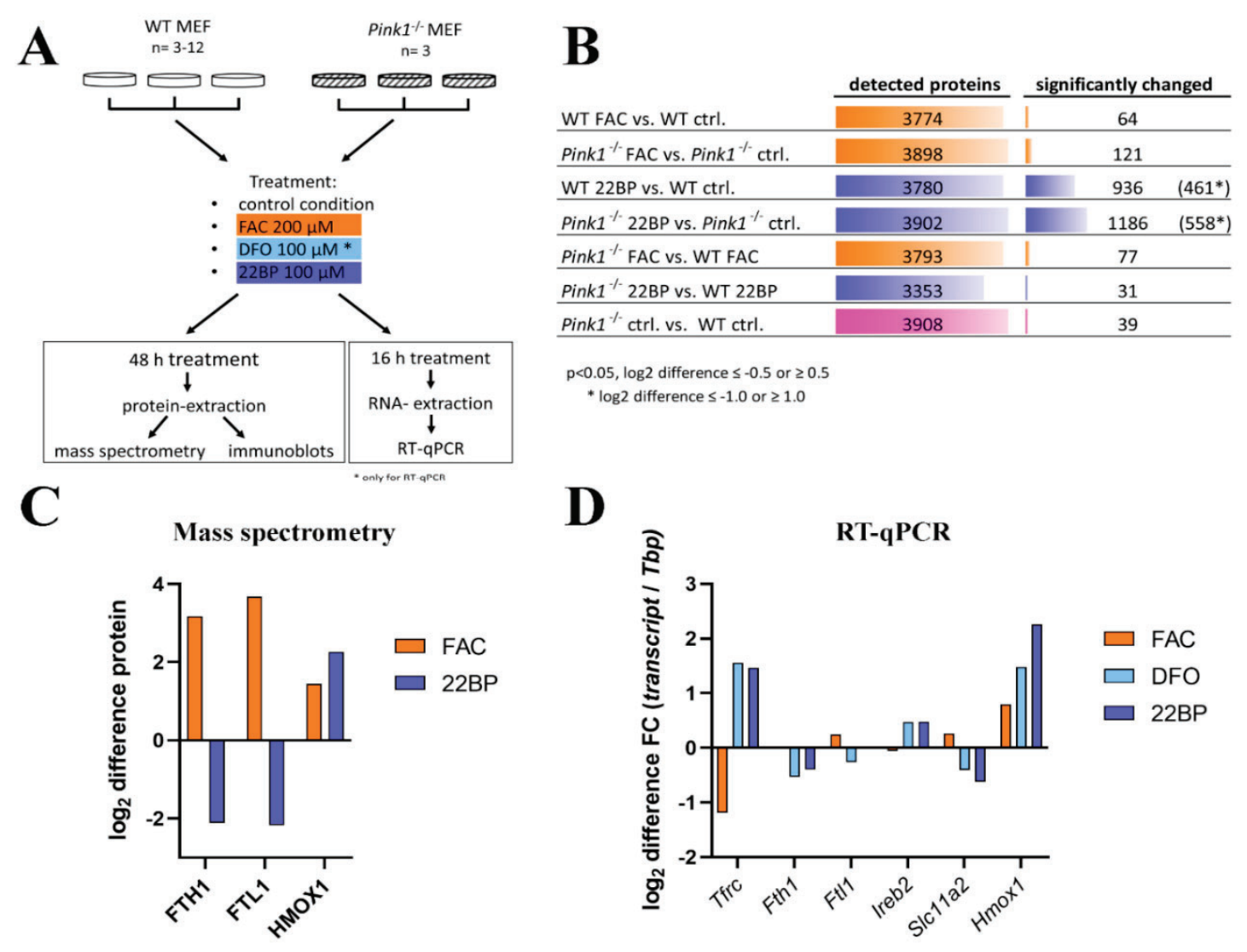

Figure 1. (A) Schematic representation of the experimental setup. Wildtype (WT) and Pink1-/mouse embryonic fibroblast (MEF) were either left untreated or incubated with ferric ammonium acid (FAC), DFO (Deferoxamine), and 2,2'-Bipyridine (22BP). mRNA expression was analyzed by RT-qPCR after $16 \mathrm{~h}$ incubation, while protein abundances were analyzed by mass spectrometry and quantitative immunoblots after $48 \mathrm{~h}$ incubation. The FAC treatment is highlighted in orange, whereas the two iron chelator treatments are highlighted in light and dark blue, as the color code for the entire manuscript. (B) Summary of mass spectrometry results for the seven conditions studied in comparison, showing the total number of detected proteins and the number of factors with significantly changed abundance, with the respective cutoff values for the significance and the fold-change (shown as $\log _{2}$ difference). The analysis of WT 22BP versus WT ctrl. and of Pink1-/- 22BP versus Pink1 ${ }^{-1-}$ ctrl. revealed so many significant factors that a $\log _{2}$ difference of 1.0 was used as the cutoff for downstream pathway enrichment analyses. (C) As measures of quality control for the culture incubations, the fold-changes as $\log _{2}$ differences are shown for well-established iron homeostasis factors, as detected in mass spectrometry, in comparison to (D) the respective $\log _{2}$ differences by RT-qPCR for such key iron homeostasis genes. The RT-qPCR results were normalized to Tbp expression levels (Tata-binding protein encoding mRNA). ctrl. = untreated control condition.

\subsubsection{FAC-Effects}

The comparison of WT MEF cells, FAC treated versus untreated, revealed an upregulation for the ferritin chain heavy subunit FTH1 and downregulation for the transferrin-receptor regulator INPP5F (Inositol Polyphosphate-5-Phosphatase F) (also known as SAC2), with additional factors showing nominal significance (see Table 1 and Figure 2A). 
Table 1. FAC effects. Overview of selected significantly changed factors in the global proteome of MEF, comparing FAC WT vs. control WT, and FAC Pink1 $1^{-/-}$vs. control Pink1 ${ }^{-/-}$, listing their respective roles and literature references (PubMedIDs), and highlighting special relevance for Parkinson's disease (PD). Upregulations are shown with dark red (FDR $>0.05)$ and light red $(p>0.05)$, whereas downregulations are marked with dark green (FDR $>0.05)$ and light green $(p>0.05)$. Factors that show converse regulation after iron overload versus iron depletion and thus appear in several tables are marked with bold fonts. Factors with dysregulation in diverse conditions are emphasized by increased table cell border thickness. For details, see Figure 2 and Supplementary Table S1.

\begin{tabular}{|c|c|c|c|}
\hline \multicolumn{4}{|c|}{ FAC WT vs. control WT (Figure 2A) } \\
\hline protein & role/relevance & reference (PMID) & $\begin{array}{l}\text { role in PD } \\
\text { (PMID) }\end{array}$ \\
\hline FTH1/FTL1 & ferritin subunits & & \\
\hline $\mathrm{XDH}$ & iron-binding purine degradation enzyme & 11132637 & \\
\hline CPT1A & mitochondrial lipid transporter & & \\
\hline MMP14 & collagen degrading factor & & \\
\hline CYB5A & ferric hemoglobin reducing cytochrome & & \\
\hline STEAP4 & vesicle-associated ferrireductase & & \\
\hline HMOX1 & heme oxygenase & & \\
\hline CISD2 & ISC-containing/ferroptosis-protecting/lifespan-modulating & 29928961,29259115 & \\
\hline MCEE & mitochondrial glyoxalase-I family member & & \\
\hline CREG1 & exocyst/exosome-regulating transcription corepressor & 30416997 & \\
\hline TRAF2 & NF-kappa-B / JNK activator, anti-apoptotic & & \\
\hline ACYP1 & apoptosis activator & 14992377 & \\
\hline INPP5F & transferrin-receptor regulator & 25869669 & \\
\hline MSRA & repair enzyme for proteins inactivated by oxidation & 31775527 & 18456002 \\
\hline PYGL & glycogen mobilizing enzyme & & \\
\hline \multicolumn{4}{|c|}{ FAC Pink1 $1^{-/}$vs. control Pink1 $1^{--}$(Figure S2A) } \\
\hline FTH1/FTL1 & ferritin subunit & & \\
\hline NSMCE2 & $\begin{array}{l}\text { metal ion binding DNA damage factor and respiration } \\
\text { regulator }\end{array}$ & 26344768 & \\
\hline JMJD6 & iron-binding histone modulator and Fech-splicing factor & 23787363 & \\
\hline MAP1LC3A & autophagosome-associated-LC3 precursor protein & & \\
\hline GPR126 & $\begin{array}{l}\text { collagen-activated CREB-regulator, G-protein coupled } \\
\text { receptor }\end{array}$ & 32219165 & \\
\hline CYB5A & ferric hemoglobin reducing cytochrome & & \\
\hline PTGES & EGLN1 interactor & 14581164,16400036 & \\
\hline STEAP4 & endosomal ferrireductase & & \\
\hline NQO1 & ROS-preventing quinone-reducing & & \\
\hline HMOX1 & heme oxygenase & & \\
\hline TRAF2 & NF-kappa-B/JNK activator, anti-apoptotic & & \\
\hline GPSM2 & nitric oxide G-protein-signaling factor & 22690686 & \\
\hline SERPINE2 & injury/ROS-responsive profibrotic endopeptidase inhibitor & 10228949 & \\
\hline ARMC1 & mitochondrial metal ion transport factor & 31644573 & \\
\hline P4HA2 & $\begin{array}{l}\text { iron-binding HIF1A-responsive collagen-folding } \\
\text { modulator }\end{array}$ & 14622280 & \\
\hline GYS1 & HIF1A-responsive glycogen synthase & 22447231 & \\
\hline COL1A1/A2 & ferroptosis-modulated fibril-forming collagen chains & 30081711 & \\
\hline MAN2C1 & metal-binding mitochondrial apoptosis modulator & 23486476 & \\
\hline TIMM10 & metal-binding mitochondrial inner membrane translocase & 19117943 & \\
\hline $\mathrm{COA} 3$ & mitochondrial assembly factor & & \\
\hline XAF1 & hypoxia-triggered mitochondrial apoptosis activator & 28507149,18485100 & 25296918 \\
\hline ETV6 & hemoglobin-transcription activator & 25281506 & \\
\hline PAWR & $\begin{array}{l}\text { oxidative stress-dependent pro-apoptotic transcription } \\
\text { repressor }\end{array}$ & & \\
\hline RBFOX2 & RNA-splicing modulator, upstream of MEF2D & & \\
\hline ZBP1 & RNA/DNA surveillance, necroptosis factor & $\begin{array}{c}17101699,9343810 \\
32296175\end{array}$ & \\
\hline GBP4 & toxic RNA sensor & 29067635 & \\
\hline OAS1G & $\begin{array}{l}\text { interferon-induced/dsRNA-activated innate immune } \\
\text { factor }\end{array}$ & 27663720 & \\
\hline EGLN1 & ferritin-modulator & & \\
\hline
\end{tabular}


A
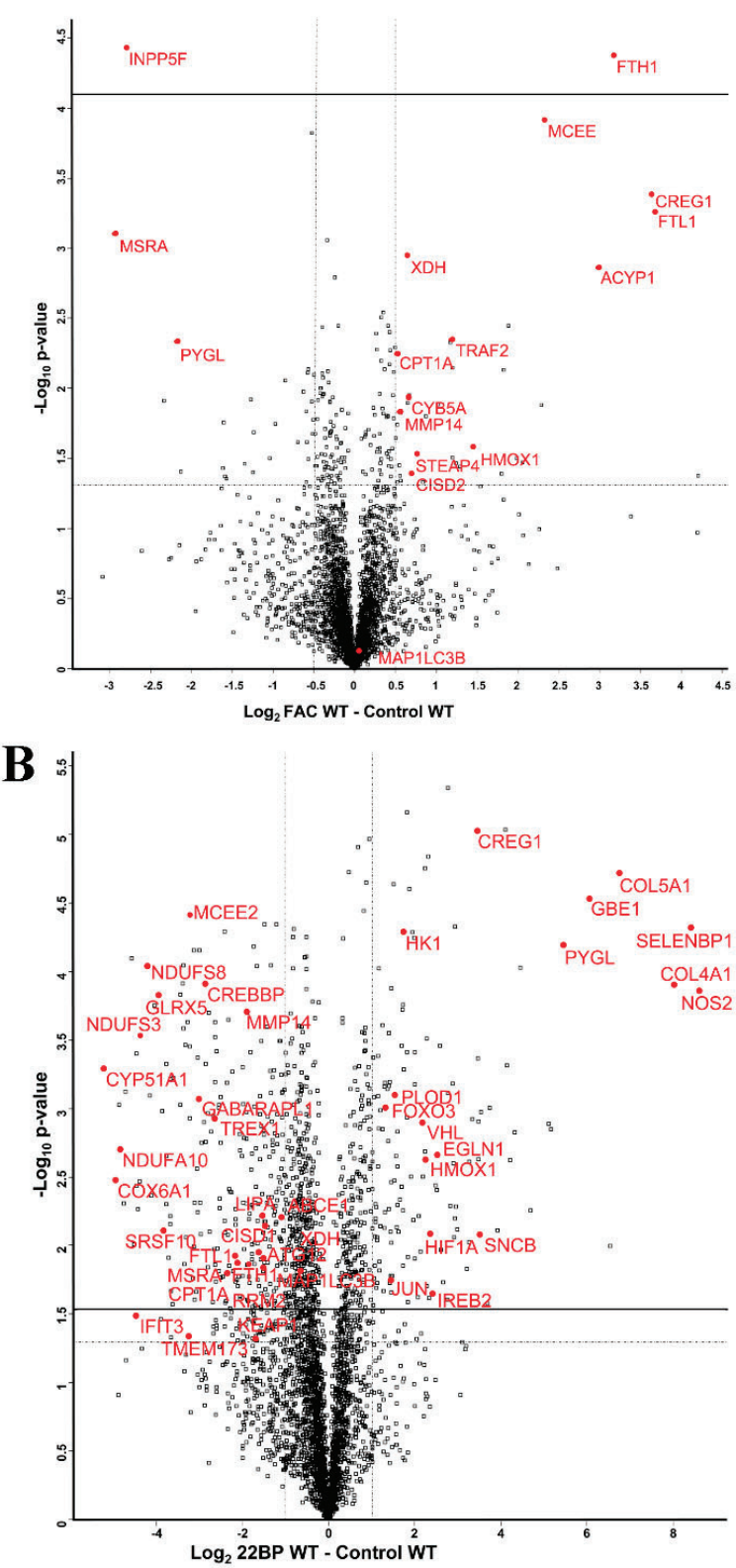

C

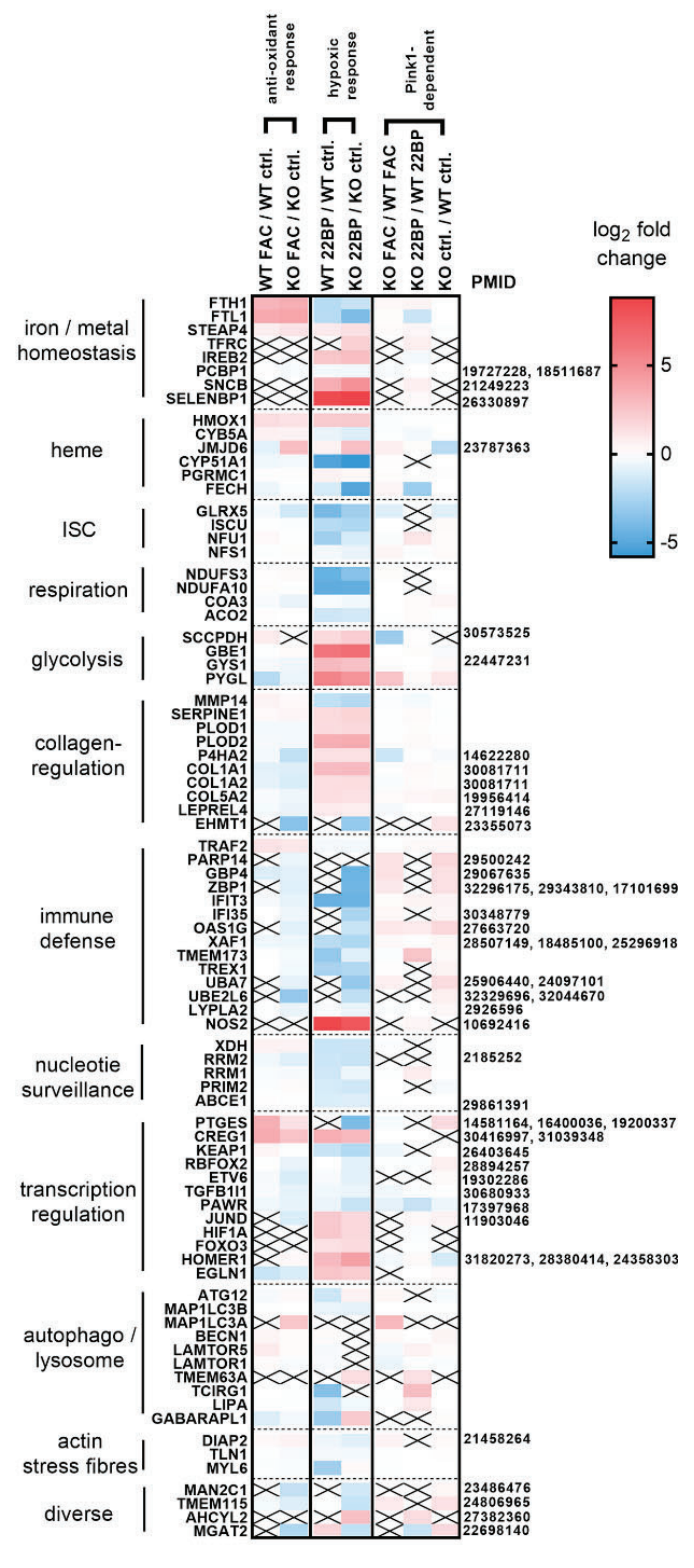

Figure 2. Global proteome profiles presented as Volcano plots, where significance ( $\log _{10} p$-value) is shown on the Y-axis (actual threshold visualized by a solid line (FDR $=0.05)$, nominal threshold by a dotted line, $p=0.05$ ), while fold-changes ( $\log _{2}$ differences) are presented on the $\mathrm{X}$-axis (dotted line refers to cutoff used for subsequent Search Tool for the Retrieval of Interacting Genes/Proteins (STRING) interaction and pathway enrichment analyses). Gene symbols were used to identify relevant proteins. Iron overload effects via FAC administration are illustrated in (A), iron depletion effects by 22BP administration in (B). The heat map in (C) summarizes the fold changes of proteins that were repeatedly dysregulated with nominal significance upon mass spectrometry, together with their categorization in pathways on the left margin, and relevant literature as PubMed database of medical literature, reference IDentifier number (PMID) on the right. $\log _{2}$ fold changes of abundance are shown in blue (negative) or red (positive), $x$ represents non-detection of the factor. ctrl. = untreated control condition.

Importantly, several factors with dysregulations after FAC treatment showed converse regulation after 22BP treatment, namely iron-binding XDH (Xanthine dehydrogenase), mitochondrial transporter CPT1A (Carnitine palmitoyltransferase 1A), collagen-degrading MMP14 (Matrix metallopeptidase 14), 
and glycogen-mobilizing PYGL (Glycogen phosphorylase L). Heat maps were generated to visualize how strongly and in which direction such factors changed after FAC versus 22BP administration, and how this was modulated by Pink1-genotype, ordered by the pathways involved and highlighting the novel impact of iron deficiency on nucleotide synthesis/surveillance factors (Figure 2C).

Overall, statistical analyses of all these factors for the enrichment of interactions, Gene Ontology (GO)-terms, pathways, and protein domains were performed with the STRING webserver [55]. This revealed multiple molecules involved in iron-binding, mitochondria, vesicles, and autophagy to show increased abundance with significant enrichment after FAC, while factors in glycolysis and metabolic pathways consistently showed decreased abundance after FAC (Supplementary Figure S1).

The comparison of Pink1 $1^{-1}$ MEF cells, FAC treated versus untreated, showed higher responsiveness (Figures $1 \mathrm{~B}$ and $2 \mathrm{C}$ ). Among so many new findings, we focused on factors with a key role for iron homeostasis, or for the mechanism of iron involvement in PD, as well as factors that are ISC- or heme-associated (Table 1, Supplementary Figure S2A). The iron-regulatory protein 2 (IRP2 aka IREB2) was not detectable after FAC treatment or in untreated condition, in WT or Pink1 ${ }^{-/-}$ cells, while it was significantly elevated after 22BP exposure. A significant upregulation of CISD2 (CDGSH iron-sulfur domain-containing protein 2) in WT cells (Figure 2A) is noteworthy in the context of post-transcriptional regulation of iron homeostasis, given the role of its homologs for the ISC regeneration in IRP1 [56-59].

It is important to note that several of these inductions are part of the known antioxidant ferroptosis-mitigating response under the control of the transcription factor NRF2 (Nuclear factor, erythroid 2 like 2, encoded by the Nfe2l2 gene), with experimental confirmation for FTL1/FTH1/HMOX1/NQO1 [NAD(P)H quinone dehydrogenase 1] [60,61]. According to GeneCards database (accessed on 31 July 2020), the NRF2 binding site exists also in the promoter of genes coding for CYB5A (Cytochrome B5 type A)/CISD2/MCEE (Methylmalonyl-CoA Epimerase)/CREG1 (Cellular repressor of E1A stimulated genes 1)/TRAF2 (TNF receptor-associated factor 2)/ACYP1 (Acylphosophatase 1)/NSMCE2 (Non-structural maintenance of chromosomes element 2 homolog)/MAP1LC3A (Microtubule-associated protein 1 light chain 3 alpha)/GPR126 (G-protein coupled receptor 126)/PTGES (Prostaglandin E synthase)/SERPINE2 (Serpin family E member 2), whereas STEAP4 (Six transmembrane epithelial antigen of the prostate)/JMJD6 (Jumonji domain protein 6)/ARMC1 (Armadillo repeat containing 1)/GPSM2 (G Protein signaling modulator 2)/MSRA (Methionine sulfoxide reductase A) promoters contain only the ARNT binding site (Aryl hydrocarbon receptor nuclear translocator) needed by the transcription factor HIF1A (Hypoxia-Inducible Factor 1-alpha) to control hypoxia responses. Indeed, among the many effects triggered by 22BP treatment in Pink $1^{-/-}$cells (Supplementary Figure S2B), dysregulations that just reached nominal significance were detected for HIF1A and the NRF2 regulator KEAP1 (Kelch-like ECH associated protein 1), in opposite direction.

The reproducibility of dysregulations between WT cells and Pink1-/- cells was assessed, and all consistent adaptations both to FAC and to 22BP were studied regarding enrichment among them for interactions, GO-terms, pathways, and protein domains, using the STRING webserver (Supplementary Figure S3). Apart from the central adaptation of iron homeostasis, ISC biogenesis, and transcription factors, this approach confirmed the impact on mitochondria, heme-containing globins, glycolysis, autophagy, and collagen. In addition, iron shortage triggered a decreased abundance of nucleotide synthesis and surveillance factors, a pathway effect that was not appreciated in previous work.

\subsubsection{BP-Effects}

Upon comparison of WT MEF cells, 22BP-mediated iron depletion triggered numerous abundance changes (see Supplementary Table S1, Table 2, Figure 2B,C). As mentioned above, FTH1-FTL1, CPT1A, MMP14, XDH, and PYGL showed converse regulation after 22BP versus FAC, whereas HMOX1 and CREG1 were upregulated in both conditions. 
Table 2. 22BP effects. Overview of selected significantly changed factors in the global proteome of MEF, comparing 22BP WT vs. control WT, and 22BP Pink1 $1^{-/-}$vs. control Pink1 $1^{-/-}$, listing their respective roles and PMIDs, and highlighting special relevance for PD. Upregulations are shown with dark red $($ FDR $>0.05)$ and light red $(p>0.05)$, whereas downregulations are marked with dark green (FDR $>$ $0.05)$ and light green $(p>0.05)$. Factors that show converse regulations after iron overload versus iron depletion and thus appear in several tables are marked with bold fonts. Factors with dysregulations in diverse conditions are emphasized by increased table cell border thickness. For details, see Figure 2 and Supplementary Table S1.

\begin{tabular}{|c|c|c|c|}
\hline \multicolumn{4}{|c|}{ 22BP WT vs. control WT (Figure 2B) } \\
\hline protein & role/relevance & $\begin{array}{c}\text { reference } \\
\text { (PMID) }\end{array}$ & $\begin{array}{c}\text { role in PD } \\
\text { (PMID) }\end{array}$ \\
\hline PYGL & glycogen mobilizing enzyme & & \\
\hline HMOX1 & heme oxygenase & & \\
\hline CREG1 & exocyst/exosome-regulating transcription corepressor & 30416997 & \\
\hline SELENBP1 & iron-interacting & 26330897 & \\
\hline IREB2 & iron-regulatory protein & & \\
\hline NOS2 & HIF1A-dependent, degradation modulator of IREB2 & $\begin{array}{l}20368827 \\
10692416 \\
\end{array}$ & \\
\hline $\begin{array}{l}\text { HIF1A/EGLN1/ } \\
\text { VHL/JUN }\end{array}$ & ferritin regulators & $\begin{array}{l}31677552 \\
11903046\end{array}$ & \\
\hline PLOD1 & iron-binding collagen crosslinking & & \\
\hline GBE1/HK1 & glycogen metabolism & & \\
\hline COL4A1/5A1 & collagen pathway & & \\
\hline FOXO3 & transcription factor, mediator of alpha-synuclein toxicity & & $\begin{array}{l}29054856 \\
19627592 \\
\end{array}$ \\
\hline SNCB & $\begin{array}{l}\text { antagonist of the toxicity of the ferrireductase } \alpha \text { - } \\
\text { synuclein }\end{array}$ & & $\begin{array}{l}21683963 \\
17556099\end{array}$ \\
\hline FTH1 / FTL1 & ferritin subunit & & \\
\hline MMP14 & collagen degrading factor & & \\
\hline CPT1A & mitochondrial lipid transporter & & \\
\hline $\mathrm{XDH}$ & iron-binding purine degradation enzyme & & \\
\hline CREBBP & ferritin regulators & & $\begin{array}{r}9794790, \\
20097775 \\
\end{array}$ \\
\hline MSRA & repair enzyme for proteins inactivated by oxidation & & 31775527 \\
\hline SRSF10 & splicing factor & & 28768533 \\
\hline GLRX5 & iron-sulfur-complex biosynthesis factor & & \\
\hline CISD1 & iron-sulfur-complex containing protein repair & & \\
\hline NDUFS3/S8/A10 & iron-sulfur respiratory complex I proteins & & \\
\hline ABCE1 & iron-binding ribosomal surveillance factor & & \\
\hline COX6A1 & iron-sensitive & 27669335 & \\
\hline CYP51A1 & heme-binding & & \\
\hline RRM2 & $\begin{array}{l}\text { ferric iron-binding ferritin-superfamily member } \\
\text { deoxynucleotide biosynthesis enzyme }\end{array}$ & & \\
\hline $\begin{array}{l}\text { GABARAPL1/LIPA/ } \\
\text { ATG12/MAP1LC3B }\end{array}$ & autophagsosomal components & & $\begin{array}{l}25767490 \\
20057503\end{array}$ \\
\hline TMEM173/ TREX1 & innate immunity components & & \\
\hline IFIT3 & toxic nucleotide sensor & & 28768533 \\
\hline KEAP1 & ferritin regulator & 26403645 & \\
\hline \multicolumn{4}{|c|}{ 22BP Pink1-/- vs. control Pink1 $1^{-/-}$(Figure S2B) } \\
\hline $\mathrm{SNCB}$ & $\begin{array}{l}\text { antagonist of the toxicity of the ferrireductase } \alpha \text { - } \\
\text { synuclein }\end{array}$ & & $\begin{array}{l}21683963 \\
17556099 \\
\end{array}$ \\
\hline FOXO3 & transcription factor, mediator of alpha-synuclein toxicity & & $\begin{array}{l}29054856 \\
19627592 \\
\end{array}$ \\
\hline GLRX5 & iron-sulfur-complex biosynthesis factor & & \\
\hline CISD1 & iron-sulfur-complex containing protein repair & & \\
\hline
\end{tabular}


Downregulations with probable relevance to PINK1 functions among the iron-binding proteins were observed for the iron-sulfur-complex biosynthesis factor GLRX5 (Glutaredoxin 5) and the iron-sulfur-complex regeneration factor CISD1 that repairs damaged IRP1. Downregulations occurred also for numerous ISC-containing factors, e.g., in the respiratory chain, as well as heme-associated proteins. Other downregulations were found for the serine and arginine-rich splicing factor SRSF10 and the toxic nucleotide sensor IFIT3 (Interferon-induced protein with tetratricopeptide repeats 3) that were implicated in PINK1-associated PD [35]. Among the factors implicated in PD pathogenesis, upregulations were found for FOXO3 (Forkhead box O3) as the mediator of toxicity of the ferrireductase alpha-synuclein (SNCA) toxicity, and for beta-synuclein (SNCB) as an antagonist of SNCA [62-66].

Statistical enrichments among all nominally dysregulated factors were identified with the STRING webserver (accessed on 16 April 2020). Among the upregulations (Supplementary Figure S4A), the hypoxia pathway, apoptosis, glucose metabolism, and collagen formation were prominently affected. Among the downregulations (Supplementary Figure S4B), the mitochondrial respiratory chain, nuclear factors, rRNA processing, and autophagosome pathways stood out. In both directions, iron response and binding factors were enriched.

Upon comparison of Pink1 ${ }^{-/}$cells MEF cells, 22BP treatment affected a myriad of factors (Figure 1B, Supplementary Figure S2B), emphasizing the strong impact of iron chelator therapy on this cell model of PD. Although the analysis of our dataset in MEF cells is important to explore the benefits and potential adverse effects of such a treatment, an exhaustive evaluation is impossible until future proteome profiles of neural cells are also available. Upon STRING enrichment analysis, changes of metabolism and ribosome/RNA factors were prominent among KEGG/Reactome pathways, with false discovery rates of $2 \mathrm{e}-36$ and $4 \mathrm{e}-27$, respectively.

Filtering those factors that responded consistently to 22BP, both in WT and also in Pink1 ${ }^{-1-}$ cells, are highlighted in Supplementary Figure S3, as e.g., GLRX5, CISD1, FOXO3, and SNCB, again with downstream effects on ISC-containing factors, e.g., in the respiratory chain, as well as heme-associated proteins. As already mentioned, a deficient abundance of several iron-binding nucleotide synthesis and RNA/DNA quality control factors appeared there as new conspicuous findings.

\subsubsection{Pink1 $1^{-/-}$Effects}

To understand the impact of a Parkinson-triggering PINK1 loss-of-function mutation among these regulations, the analogous experiments with $\mathrm{FAC} / 22 \mathrm{BP}$ administration were performed in comparisons between Pink1 $^{-/-}$versus WT MEF cells (Supplementary Table S1, Table 3).

Table 3. PINK1 effects. Overview of selected significantly changed factors in the global proteome of MEF, comparing FAC Pink1-/- vs. FAC WT, 22BP Pink1 $1^{-/-}$vs. 22BP WT, and control Pink1 $1^{-/-}$ vs. control WT, listing their respective roles and PMIDs, and highlighting special relevance for PD. Upregulations are shown with dark red (FDR $>0.05)$ and light red $(p>0.05)$, whereas downregulations are marked with dark green (FDR $>0.05)$ and light green $(p>0.05)$. Factors that show converse regulations after iron overload versus iron depletion and thus appear in several tables are marked with bold fonts. Factors with dysregulations in diverse conditions are emphasized by increased table cell border thickness. For details, see Figure 2 and Supplementary Table S1.

\begin{tabular}{|l|l|c|c|}
\hline \multicolumn{4}{|c|}{ FAC Pink1-/- vs. FAC WT (Figure S2C) } \\
\hline \multicolumn{1}{|c|}{ protein } & \multicolumn{1}{|c|}{ role/relevance } & reference (PMID) & role in PD (PMID) \\
\hline MAP1LC3A & precursor protein of LC3A for Parkin-independent mitophagy & 19794493 & 32442087,28381481 \\
\hline SLC25A11 & mitoch. fusion modulator, via oxoglutarate controls OGFOD1 & 26118662,24550447 & 25294124 \\
\hline PYGL & glycogen mobilizing factor & & \\
\hline MYD88 & innate immunity signaling coordinator & & \\
\hline IRF2BP1 & immunity transcriptional corepressor & & 21812969 \\
\hline SPP1 & immunity cytokine & & \\
\hline
\end{tabular}


Table 3. Cont.

\begin{tabular}{|c|c|c|c|}
\hline NUCKS1 & positive transcriptional regulator of insulin signaling & 24931609 & 28108469 \\
\hline PANK4 & neuroprotective upon excess acetyl-CoA production & 27322068,19602483 & \\
\hline MYL6 & $\begin{array}{l}\text { putative interactor of the iron chaperone PCBP1 } \\
\text { that delivers ferrous ion to ferritin }\end{array}$ & 19727228,18511687 & \\
\hline GYPC & hemin-interactor & 8220237 & \\
\hline SCCPDH & mitochondrial dynamics factor & 30573525 & \\
\hline HMGCL & mitochondrial ketogenesis regulator & & \\
\hline PMPCA & mitochondrial signal peptide cleavage factor & & \\
\hline LAMTOR1 & lysosomal amino acid sensor & & \\
\hline ABCB1 & lipid transporter & & 29224383,19732497 \\
\hline AGPAT1 & lipid enzyme, target of HIF1A & 29908837 & \\
\hline MRPS36 & citric-acid-cycle component and mitoribosomal subunit & 25165143 & \\
\hline RPF2 & ribosome assembly factor & & \\
\hline BUD31 & core spliceosome component & 26331541 & \\
\hline \multicolumn{4}{|c|}{ 22BP Pink1 $1^{-/-}$vs. 22BP WT (Figure S2D) } \\
\hline ARPP19 & cAMP-regulated DNA-damage sensor and mitosis initiator & & \\
\hline SRSF10 & splicing factor & & 28768533 \\
\hline CREBBP & circadian transcriptional coactivator & & 20097775 \\
\hline RRM1 & interactor of RRM2, DNA-repair enzyme & & \\
\hline UPP1 & uridine degradation enzyme & & \\
\hline LBH & transcriptional activator & & \\
\hline IKBKAP & transcript elongation factor & & \\
\hline EXOSC $4 / 10$ & RNA surveillance factors & & \\
\hline AHCYL2 & $\mathrm{pH}$ regulator & 27382360 & \\
\hline NMRAL1 & redox sensor & & \\
\hline CCDC58 & mitochondrial adaptor & 22851751 & \\
\hline MRPS36 & $\begin{array}{l}\text { mitochondrial } \alpha \text {-ketoglutarate dehydrogenase complex } \\
\text { adaptor, mRNA translation factor }\end{array}$ & 25165143 & \\
\hline LIPA & lysosomal enzyme & & \\
\hline TCIRG1 & lyso/endosomal proton pump with role in iron import & & \\
\hline TMEM63A & lysosomal cation channel & & \\
\hline LAMTOR5 & lysosome-associated amino acid sensor & & \\
\hline HMOX1 & heme oxygenase & & \\
\hline AMDHD2 & $\begin{array}{l}\text { complex carbohydrate degradation enzyme } \\
\text { immune defense factor }\end{array}$ & 2307398,11705974 & \\
\hline FECH & heme-iron enzyme & & \\
\hline CYB5A & cytochrome B 5A reduces ferric to ferrous hemoglobin & & \\
\hline ACSL3 & beta-oxidation factor & & \\
\hline MRPS35 & mitochondrial mRNA translation factor & & \\
\hline ATG4B & autophagy enzyme, cleaves MAP1LC3B / GABARAPL1 & 14530254 & \\
\hline SXN30 & autophagy-related sorting & & \\
\hline \multicolumn{4}{|c|}{ Control Pink1 ${ }^{-/-}$vs. control WT (Figure S2E) } \\
\hline DIP2A & hypoxia-associated acetyl-CoA biogenesis factor & 31600191,2005400 & \\
\hline RBFOX2 & RNA-splicing factor and stress granule component & & \\
\hline TMEM115 & retrograde vesicle transport factor & & \\
\hline BECN1 & autophagy factor & & 20057503,24879156 \\
\hline SYN1 & nitric oxide synthase interactor & 24358303 & \\
\hline NUP160 & autophagy modulator, poly(A)+ RNA nucleoplasmic transport & 29704630,11684705 & \\
\hline GLRX5 & iron-sulfur-complex biogenesis factor & & \\
\hline PDDC1 & PARK7-homologous peptidase & & \\
\hline HOMER1 & calcium-excitation factor & & 24358303 \\
\hline
\end{tabular}


Table 3. Cont.

\begin{tabular}{|l|l|c|l|}
\hline POMP & ubiquitin-independent 20S proteasome assembly factor & 31742837 & \\
\hline PCBP3 & ferritin-interactor & 23640898 & \\
\hline JMJD6 & the iron-binding histone modulator and FECH-splicing factor & 23787363 & \\
\hline ATG12 & autophagy factor, ubiquitin-like & & \\
\hline
\end{tabular}

After iron overload with FAC (Supplementary Figure S2C), the Pink1 ${ }^{-/-}$cells showed upregulations of MAP1LC3A probably as an effort to promote Parkin-independent mitophagy [67], and of SLC25A11 (Solute carrier family 25 member 11)/PANK4 (Pantothenate kinase 4)/PYGL/NUCKS (Nuclear casein kinase and cyclin dependent kinase substrate 1) as evidence for excessive mitochondrial metabolic performance, as well as innate immune responses such as SPP1 (Secreted phosphoprotein 1 possibly due to mitochondrial accumulation in this PD variant [68-70]. Downregulations occurred for the myosin light chain MYL6 as a putative interactor of the iron chaperone PCBP1 (Poly(RC) binding protein 1), as well as several mitochondrial and lysosomal factors, while the affection of ribosomal and spliceosomal factors like RPF2 (Ribosome production factor 2 homolog) and BUD31 (BUD31 homolog) stood out as novel insights (Table 3).

After iron depletion with 22BP (Supplementary Figure S2D), the Pink1 ${ }^{-/-}$cells showed notable downregulations of the autophagy factor ATG4B (Autophagy related 4B cysteine peptidase), responsible for cleavage of MAP1LC3A and its homologs, as well as dysregulations of 3 heme-associated factors (ferrochelatase (FECH), HMOX1, cytochrome B5 type A (CYB5A)). Relevant upregulations were detected for the CAMP-regulated DNA-damage sensor and mitosis initiator ARPP19 (cAMP regulated phosphoprotein 19), the splicing factor SRSF10 that was previously identified as PINK1-regulated [35], the circadian transcriptional coactivator CREBBP (CREB binding protein) that was implicated in PD [71], and the DNA-repair factor RRM1 (Ribonucleotide reductase regulatory subunit) that interacts with ferritin superfamily member RRM2 (Table 3).

During untreated culture conditions, the Pink1 ${ }^{-/}$cells exhibited an upregulation (Supplementary Figure S2E) for the autophagy factor BECN1 (Beclin 1) in a probable effort to facilitate Parkin-dependent mitophagy, together with downregulation for the ubiquitin-like autophagy factor ATG12 (Autophagy related 12). Other downregulations were notable for the iron-sulfur-complex biogenesis factor GLRX5, the iron-binding histone modulator and $F E C H$-splicing factor JMJD6, and the ferritin interactor PCBP3, suggesting a chronic PINK1 impact on ISC/heme biogenesis and LIP even in conditions without stress. Further downregulations occurred for the PARK7-homologous peptidase PDDC1 (Glutamine amidotransferase-like class 1 domain containing 1; which interacts with the ferroptosis-induced CREB-associated PRKN-regulator TRIB3 (Tribbles pseudokinase 3)), and for the calcium-excitation factor HOMER1 (Homer scaffold protein 1) that was implicated in PD.

Overall, these data tentatively identify Pink1 $^{-/-}$effects on 8 iron/ISC/heme homeostasis factors (TCIRG1 (T Cell immune regulator 1), MYL6, PCBP3, GLRX5, FECH, JMJD6, CYB5A, GYPC (Glycophorin C)), 8 unspecific mitochondrial factors (SLC25A11, SCCPDH (Saccharopine dehydrogenase), HMGCL (Mitochondrial 3-Hydroxy-3-Methylglutaryl-CoA Lyase), PMPCA (Peptidase, mitochondrial processing subunit alpha), MRPS35 (Mitochondrial ribosomal protein S35, MRPS36 (Mitochondrial ribosomal protein S36), CCDC58 (Coiled-coil domain containing 58), ACSL3 (Acyl-CoA synthetase long-chain family member 3)), 6 autophagy factors (MAP1LC3A, ATG4B, ATG12, ATG24A, BECN1, NUP160 (Nucleoporin 160)), 5 lysosomal factors (LAMTOR1 (Late endosomal/lysosomal adaptor 1), LIPA (Lipase A) TMEM63A (Transmembrane protein 63A), LAMTOR5 (Late endosomal/lysosomal adaptor 5), TCIRG1), 5 RNA splicing and surveillance modulators (BUD31, SRSF10, EXOSC4, EXOSC10 (Exosome component 4/10), RBFOX2 (RNA binding fox-1 homolog 2)), 5 DNA-associated/-repair factors (NUCKS, ARPP19, CREBBP, RRM1, UPP1 (Uridine phosphorylase 1)), and 4 innate immunity factors (MYD88 (Myeloid differentiation primary response protein MyD88), IRF2BP1 (Interferon regulatory factor 2 binding protein 1), SPP1, AMDHD2 (Amidohydrolase domain containing 2)). This list is in good agreement with the known functions 
of PINK1 in mitochondrial degradation via the autophago-lysosomal pathway and with the iron accumulation in PINK1-mutant brain. There was no Pink1-dependent factor with converse regulation during iron excess versus iron shortage.

\subsection{Transcriptional Analyses of Cellular Iron Homeostasis Factors in WT MEF in Response to Altered Iron Levels}

Global proteomics by mass spectrometry usually detects less than 10,000 proteins. However, this represents only a minor fraction of all proteins in MEF cells. The human genome project identified about 20,000 genes, each of which encodes up to 10 proteins, leading to current estimates of 80,000-400,000 proteins within the human body and probably also in the mouse. Proteins with low abundance, integration into membranes, complexes with nucleotide chains may not be detected easily. For a systematic evaluation of all core events in iron homeostasis, we used RT-qPCR to target all relevant factors and assess their transcriptional changes after $16 \mathrm{~h}$ in three conditions. Firstly, WT MEF cells were employed in a discovery phase (this paragraph, with Figure 3 and Supplementary Figure S5 illustrating expression adaptations; Supplementary Table S2 provides fold-changes with significance values). Secondly, Pink1 ${ }^{-/}$MEFs were used (subsequent paragraph 2.3) to test reproducibility versus genotype-dependent alterations.

In the discovery phase of this project, we wanted to account for biological variability and screened a large number of WT MEF for iron-dependent expression regulations (plain bars in Figure 3 and Supplementary Figure S5). To test reproducibility, the iron chelator drug DFO was used as an alternative to the iron chelator 22BP. The novel observations regarding transcriptional induction of mitophagy factors Pink1 and Prkn (encoding the protein PARKIN) upon iron depletion is documented in Figure 3A. As further proof-of-principle for the iron-responsiveness of fibroblast expression profiles, Figure 3B shows the transcriptional response of the transferrin receptor (Tfrc), which is the main receptor to import iron into the cell. Also shown is the iron storage ferritin chain with its heavy subunit (Fth1) and light subunit (Ftl1), as well as the small subunit of ribonucleotide reductase (Rrm2), as $\mathrm{Fe}^{3+}$ binding ferritin superfamily member. Tfrc appeared reduced only to 0.45 -fold under iron excess conditions $(p=0.7155)$, again suggesting that iron levels in the basal culture medium were already so high that transferrin-receptor expression could not be downregulated much during FAC administration. Tfrc was highly induced upon iron deficiency. Thus, the main regulator of iron uptake responded to iron depletion in a sensitive manner. Fth 1 and $F t l 1$ were not changed significantly and displayed high variability among the 9 different WT MEF cell lines, but both ferritin subunit transcripts showed about 1.5-fold higher levels during iron excess. Conversely, Rrm2 as a deoxynucleotide biosynthesis enzyme exhibited significantly lower levels during iron depletion. Slc40a1 mRNA encoding ferroportin-1 as cellular iron exporter appeared with levels below 0.45-fold during iron depletion (Supplementary Figure S5). These experiments confirmed that MEF cells are responsive to manipulation of iron availability, so we performed further studies into their adaptations of mRNA expression, focusing on all crucial factors of the cellular iron transport and mitochondrial homeostasis, of the heme synthesis pathway and hemeproteins, of the ISC biogenesis pathway and ISC-containing factors.

There were various factors related to these pathways that exhibited no significant dysregulations under any condition or were less relevant or redundant, so they were summarized in Supplementary Figure S5. In alphabetical order, these factors include $A b c b 6$ (ATP binding cassette subfamily B member 6), Aco1 (Aconitase 1), Aco2 (Aconitase 2), Alas1 (5'-aminolevulinic acid), Bach1 (BTB domain and CNC homolog 1), Bdh2 (3-hydroxybutyrate dehydrogenase-2), Brip1 (Brca1 interacting protein C-terminal helicase 1), Cdc42bpa (Cdc42 binding protein kinase alpha), Cisd1, Cisd2, Cp(Ceruloplasmin), Cygb, Dpyd (Dihydropyrimidine dehydrogenase), Elp3 (Elongator acetyltransferase complex subunit 3), Ercc2 (ERCC excision repair 2), Fdx1 (Ferredoxin 1), Fech, Flvcr1 (Feline leukemia virus subgroup C cellular receptor 1a), Fxn (Frataxin), Hebp1, Myl6, Ncoa4 (Nuclear receptor coactivator 4), Pcbp1, Pcbp2, Prim2 (DNA primase subunit 2), Rsad1, Rsad2 (Radical S-adenosyl methionine domain containing 1/2), Rtel1 (Regulator of telomere elongation helicase 1), Slc25a37, Slc40a1, Steap3, and Trf (Transferrin). 
The other factors with important dysregulation are shown in the main figures and are individually mentioned in Table $\mathrm{S} 2$ as well as the text below, together with their respective roles.

Tfrc mRNA is stabilized by IREB2, whose mRNA was also induced after iron deprivation (Figure 3C). After transferrin binding to TFRC protein and subsequent endocytosis of this complex, the acidic $\mathrm{pH}$ in endosomes releases ferric iron $\left(\mathrm{Fe}^{3+}\right)$, which is reduced to the ferrous form $\left(\mathrm{Fe}^{2+}\right)$ by the metalloreductases STEAP2 and STEAP3. Iron molecules then get exported by the Divalent metal transporter 1 (DMT1 encoded by Slc11a2) to the cytosol [4,72]. After iron overload, Steap2 and Slc11a2 mRNA showed a significant average 1.25-fold upregulation (Figure 3C, similar to Steap3 in Supplementary Figure S5), indicating higher biosynthesis of the factors responsible for the reduction and export of iron. During both forms of iron depletion, Steap2 and Slc11a2 mRNAs were significantly reduced to 0.7 -fold, so their levels are in direct correlation with iron availability.

The ISC-containing 3-hydroxybutyrate dehydrogenase-2 (BDH2) catalyzes the rate-limiting step in the biosynthesis of siderophores, which are soluble $\mathrm{Fe}^{3+}$ binding agents [73]. Upon iron depletion, Bdh2 transcription also was downregulated to 0.7-fold (Supplementary Figure S5). BDH2 inhibition was shown to result in cellular iron accumulation [74], so again its expression adaptation could represent a homeostatic effort to increase intracellular iron levels.

The poly(RC)-binding-protein-2 (PCBP2) is involved in mRNA metabolism and translation, as well as innate immune signaling, but was previously shown to function also as a chaperone for the LIP in the cytosol and to interact with HMOX1 [75]. However, neither Pcbp2 nor Pcbp1 transcripts were consistently changed by iron level manipulation (Supplementary Figure S5). Interestingly, the mRNA of Pcbp3 was reduced to 0.5-fold after iron depletion, emphasizing its role in iron metabolism as the PCBP family member with the strongest ferritin interaction [76].

The iron regulatory proteins (IRP1 encoded by the Aco1 mRNA, and IRP2 by Ireb2) sense cytosolic iron availability and ensure adequate iron supply to mitochondria [77], firstly via association with iron response elements (IREs) in the untranslated region (UTR) of ferritin Fth1/Ftl1 mRNA to inhibit its translation, and secondly via association with iron importers Tfrc/Slc11a2 mRNA to stabilize them and facilitate their translation when cellular iron levels are low. During both forms of iron depletion, the transcription of Ireb2 showed a 1.4-fold upregulation, while Aco1 showed a consistent reduction to 0.6-fold (Supplementary Figure S5). Under conditions of sufficient iron, IRP1 exerts its cytosolic aconitase functions while IRP2 gets degraded, resulting in converse effects with increased ferritin translation and TFRC degradation [78]. After iron overload, we detected no relevant expression adaptation of both transcripts encoding iron regulatory proteins.

Expression of the mitochondrial inner membrane transporter Slc25a28, which encodes Mitoferrin-2 (MFRN2), did not react after iron overload but was significantly reduced to 0.6-fold after iron depletion. Mitoferrin-1 (Slc25a37 or Mfrn1), however, did not show altered expression upon iron level manipulation in WT cells (Supplementary Figure S5). MFRN1 forms a complex with the mitochondrial inner membrane iron transporter ABCB10 [72,79]. Abcb10 transcript levels were 1.4-fold higher under both iron depletion conditions, reaching significance for DFO. Given that it is not clear how these two inner membrane transporters of iron are acting in a complementary fashion, it is interesting to note that $A b c b 10$ mRNA was higher during low iron conditions, as expected for an iron uptake factor. However, Slc25a28 mRNA was diminished under the same treatment, a response that would be in line with an iron export factor. In comparison, $A b c b 7$ and $A b c b 8$ (ATP binding cassette subfamily B member $7 / 8$ ) are thought to have mitochondrial export functions [72,80-82] and are also important for heme biosynthesis [83]. As expected after iron depletion, $A b c b 7$ transcript levels were halved, similar to Abcb8 (Figure 3). 


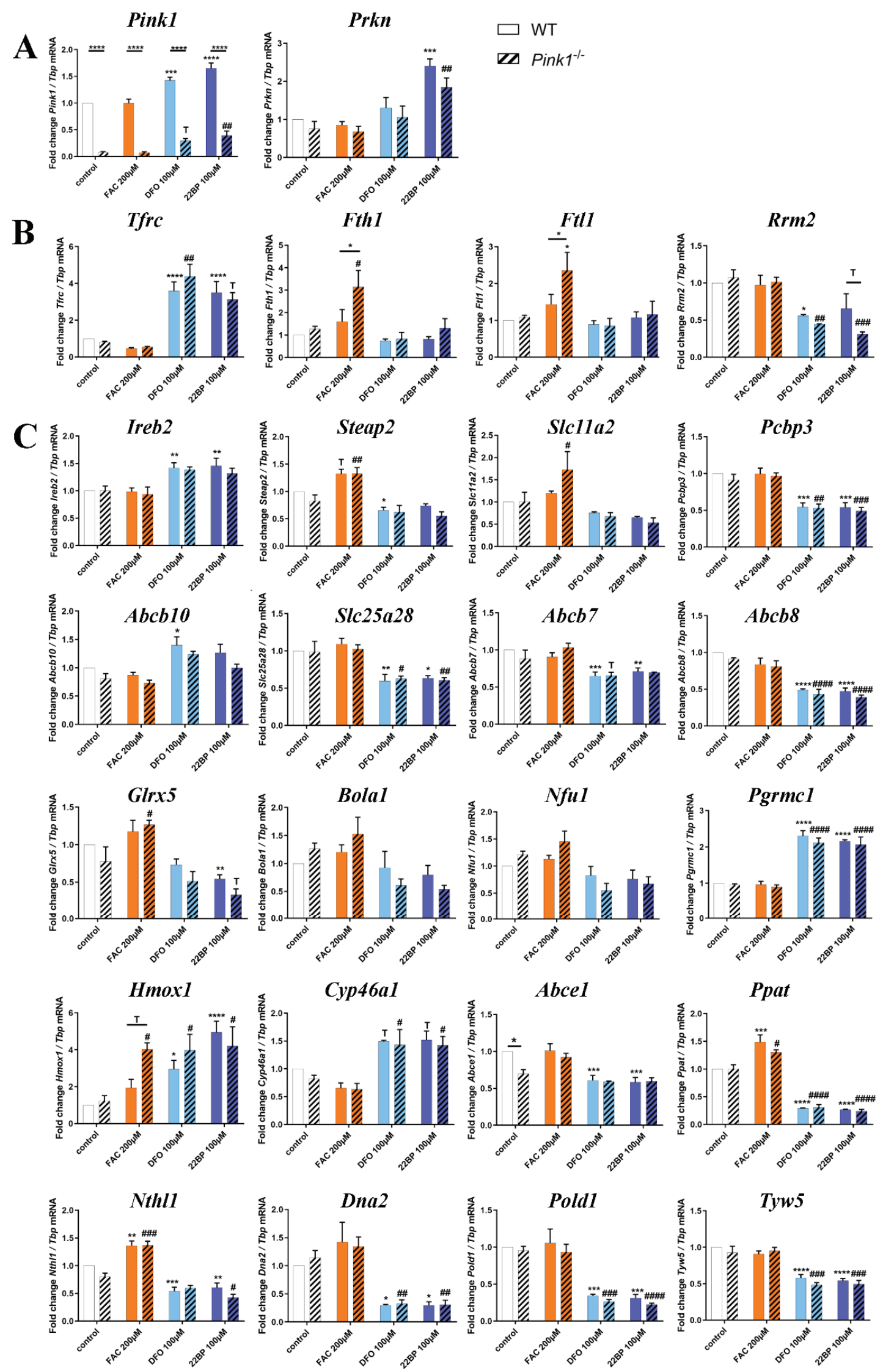

Figure 3. Synopsis of mRNA expression dysregulations upon RT-qPCR in MEF cells ( $n=3 \mathrm{WT}$ for Pink1 and Prkn, else 6-12 WT versus 3 Pink $1^{-/-}$for all other factors) after iron manipulation, focusing 
on (A) the mitophagy modulators Pink1 and Prkn, (B) key modulators of cellular iron uptake, storage, export, (C) iron transport and processing, mitochondrial iron homeostasis, heme production/turnover, ISC-biogenesis, and ISC-binding. All factors in $(\mathbf{B}, \mathbf{C})$ are presented in their approximate order of action during cellular iron homeostasis. Their expression adaptation was documented after iron overload (FAC) and under two different iron depletion conditions (DFO, 22BP), after normalization to Tbp expression levels as the loading control. Mean values with SEM (standard error of the mean) are shown, normalized to the WT control condition. The statistical trends or levels of significance are illustrated by symbols, namely T: $0.1>p>0.05,{ }^{*}$ or \#: $p<0.05,{ }^{* *}$ or \#\#: $p<0.01,{ }^{* * *}$ or \#\#\#: $p<0.001,{ }^{* * * *}$ or \#\#\#\# $p<0.0001$. Mutant cells are represented by dashed bars, WT cells by plain colors. Asterisks are used for WT MEF to represent significant effects between treated and untreated control cells, while hashtags refer to Pink1 $1^{-/}$MEF with significant differences between treated versus untreated control cells. Genotype-dependent significant differences of Pink1 ${ }^{-/-}$versus WT MEF are illustrated by horizontal lines below asterisks. Detailed fold-changes and $p$-values are listed in Supplementary Table S2.

Once transported into the mitochondrial matrix, iron may be stored or used for the biosynthesis of ISC and heme [84]. The mitochondrial iron storage factor mitochondrial ferritin was not detectable in MEF cells under the conditions tested. For biosynthesis purposes, iron is incorporated by FXN into the complex containing sulfur-containing NFS1 (Nitrogen fixation 1 homolog), ISCU (Iron-sulfur cluster assembly enzyme), and LYRM4 (LYR motif containing 4) to generate [2Fe-2S] clusters, which associate with GLRX5 and the assembly factor BOLA1 (BolA Family Member 1) [3,85,86]. Subsequently, these ISC are transferred by the ISC scaffold NFU1 (Nfu1 iron-sulfur cluster scaffold) to target proteins [87]. After both iron depletion conditions, Glrx 5 expression was reduced to 0.5 -fold. Fxn, Bola1, and Nfu1 levels were around 0.7-fold after iron depletion, but this was not statistically significant. The converse iron overload did not modulate expression of the ISC biogenesis proteins.

Pursuing the heme- and ISC-associated pathways into the cytosol, it is relevant that the putative heme release factor Pgrmc1 (Progesterone receptor membrane component 1), as a cytosolic factor in association with mitochondrial ferrochelatase, showed a highly significant and more than two-fold transcriptional induction after iron depletion. Heme oxygenase 1 (HMOX1) acts to degrade cytoplasmic heme by cleaving it to biliverdin, as the rate-limiting step of heme breakdown [88]. After iron depletion, also Hmox1 mRNA was strongly upregulated with high significance. These upregulations might reflect compensatory cellular efforts to recruit iron via heme breakdown. The cholesterol elimination factor CYP46A1 (Cytochrome P450 family 46 subfamily A member 1) belongs to the Cytochrome P450 family, which is known to bind heme as a co-factor [89,90]. After iron depletion, Cyp46a1 transcript showed a statistical trend towards a 1.5-fold increase; it was not altered after iron overload. Continuing the heme-related pathway, the transcript levels of Cytoglobin (Cygb) [91] were quantified, as one vertebrate globin family that is expressed in fibroblasts, but they showed no change and high variability (Supplementary Figure S5).

Not only heme but also ISC are incorporated into target proteins, many of which have nucleotide processing functions. Among the ISC-containing factors present inside and outside of mitochondria, the essential ribosome recycling factor ABCE1 (ATP binding cassette subfamily E member 1) exerts crucial functions to avoid an accumulation of ribosomes at the stop codon, inefficient ribosomal cycling, and stalled translation [92,93]. Interestingly, after both forms of iron depletion, a significant decrease to 0.6-fold was observed for Abce1 mRNA, while iron overload triggered no expression adaptation.

The ISC-containing phosphoribosyl pyrophosphate amidotransferase (PPAT = GPAT) is the rate-limiting enzyme in de novo purine nucleotide biosynthetic pathways. Its expression showed a strong direct correlation of high significance with the availability of iron. After incubation with FAC, Ppat was significantly upregulated 1.5-fold. After iron depletion, it was downregulated with high significance to 0.3 -fold levels. Both sensitive adaptations provide evidence that iron is important for nucleotide homeostasis. 
The Nth like DNA glycosylase 1 (NTHL1) has relevant functions in base excision repair, harbors an ISC, and is localized both in the nucleus and the mitochondrial matrix. The expression of Nthl1 reacted similarly to Ppat with significant upregulation after iron overload versus downregulation after both forms of iron depletion, although with less effect size and less significance.

Similarly, three other ISC-associated nuclear factors implicated in DNA quality control also showed consistent downregulations after iron deprivation, namely the DNA replication helicase Dna2, the DNA primase subunit Prim2 (Supplementary Figure S5), and the DNA polymerase delta subunit Pold1. Also, the ISC-containing Elongator complex protein 3 (ELP3), which acts in tRNA modification [94], showed significantly lower transcript levels to 0.7-fold after iron depletion (Supplementary Figure S5).

The tRNA wybutosine synthesizing protein 5 (TYW5) was reported to catalyze a carbon hydroxylation using $\mathrm{Fe}^{2+}$ ions as cofactors, so its activity depends on iron levels. Tyw5 expression did not react to iron overload, but it was significantly downregulated after iron depletion.

Jointly, these results in WT cells indicate that upon iron overload and even more upon iron depletion, transcriptional expression adapts up to two-fold within $16 \mathrm{~h}$ for specific iron homeostasis factors at the plasma membrane, in endosomes and the cytosol, inside mitochondria as well as the nucleus. A negative correlation was found for iron transport components at the plasma membrane and mitochondrial membrane, which responded to iron depletion with induced expression of Tfrc/Ireb2 and Abcb10, respectively. Similarly, the iron recruitment option via mitochondrial heme release and its cytosolic breakdown responded to iron depletion with induced expression of Pgrmc1/Hmox1. Furthermore, the increased need for the heme-binding cholesterol catabolism enzyme Cyp46a1 seemed apparent during iron depletion, since its transcript levels were consistently elevated. In contrast, a strong direct positive correlation with iron levels was observed for endosomal and cytosolic iron processing factors Steap2 (less for Slc11a2), exhibiting increased expression upon iron overload versus decreased expression upon iron depletion. A similarly strong direct correlation was also documented for ISC-containing factors of nucleotide metabolism, namely Ppat and Nthl1. All other expression adaptations observed simply reflected the diminished synthesis of iron-associated factors under conditions of low iron levels.

\subsection{Transcriptional Analyses of Expression Adaptations of Pink1 $1^{-/}$MEF to Altered Iron Levels}

As a validation effort and to obtain additional mechanistic insights in this project, experiments were performed in 3 Pink1-deficient MEF lines where mitophagy is impaired. Again, FAC-mediated iron overload and DFO/22BP-mediated iron depletion were studied. With this approach, we hoped to elucidate how iron, ISC, and heme homeostasis interdepend with mitochondrial turnover.

Figure 3A shows mRNA levels of Pink1 and Prkn as regulators of mitophagy. The bars hatched in black within the first panel confirm the knockout of Pink1 in the 3 MEF lines and reproduce the high transcriptional induction of Pink1 after iron deficiency (see also Supplementary Table S2). This transcript induction was much less strong in the Pink1 $1^{--}$cells than in WT cells. These Pink1 ${ }^{-/-}$MEF derive from a mouse where an intron is retained in the Pink1 $1^{-/}$mRNA and triggers a changed reading frame, so Pink1 mRNA is rapidly degraded and the PINK1 protein is absent, but the Pink1 promoter is still actively responding to specific stressors like iron depletion. Similarly, the Prkn transcript again got induced upon iron depletion. These results in Figure 3A corroborate the concept that PINK1/PARKIN-dependent mitophagy gets highly induced after iron starvation in MEF. We showed previously in the neuronal cell line SH-SY5Y that nutrient starvation by HBSS medium (which includes transferrin deprivation) will induce Pink1 and Prkn transcript expression and protein abundance together with activation of mitophagy and lysosomal degradation, by the use of recombinant tagged PARKIN-constructs [19].

Overall, the comparison of PINK1-deficient cells, treated versus untreated, reproduced many observations (see significance symbols above hatched bars in Figure 3, numeric values in Supplementary Table S2 and the overall pattern in Supplementary Figure S5): Again, iron depletion induced the mitophagy factors Pink1, Prkn, the iron import factor Tfrc, and factors for iron-release from heme 
such as Pgrmc1, Hmox1 with Cyp46a1, while it reduced the siderophore biosynthesis factor Bdh2, the mitochondrial iron homeostasis factors $S l c 25 a 28, A b c b 7, A b c b 8$, and Glrx 5, as well as the nucleotide surveillance factors Ppat, Nthl1, Dna2, Pold1, Tyw5, and Elp3. Reproducibly, iron excess induced the iron reductase Steap2, as well as the nucleotide surveillance factors Ppat and Nthl1. These findings constitute a validation of the regulations in WT cells.

It is crucial to note that several regulations lost or reached significance in Pink1 $1^{-/} \mathrm{MEF}$, compared to WT MEF. A schematic overview of all significant iron effects and trends to regulate the expression of its homeostasis factors at their mRNA levels is provided in Figure 4. Consistently, the cells with PINK1-absence (a state that occurs physiologically when mitochondria are healthy) were less responsive to iron depletion for mRNA adaptations. This rule concerned the plasma membrane Tfrc, cytosolic Ireb2, Steap2, Bdh2, Abce1, and mitochondrial Abcb10 expression; conversely, the cells with PINK1-absence were reacting more strongly to iron excess. This pattern was visible for cytosolic factors Fth1, Ftl1, Hmox1 with significance or trend, and similarly without significance for cytosolic Slc11a2, Bdh2, Aco1, as well as mitochondrial Slc25a37 and Glrx5 regulations. These mRNAs may be elevated due to enhanced transcript synthesis or due to enhanced transcript stability. Fth1, Ftl1, and Slc11a2 mRNAs have regulatory elements in their $5^{\prime}$ UTR that modulate their translational repression by IRP1/ACO1 and IRP2/IREB2 under influence of the LIP (which is modulated by BDH2), whereas the Hmox1 mRNA contains regulatory elements to modulate its translational repression under the influence of heme concentrations $[95,96]$. Furthermore, there are known functional interactions between cytosolic IRP1/ACO1 and mitochondrial SLC25A37 as well as GLRX5 [57,97]. In contrast, most other mitochondrial effects did not depend on PINK1 presence, nor did nuclear effects, except for the significant reduction of Prim 2 upon iron deprivation (Supplementary Figure S5). These observations provide preliminary evidence that PINK1 biosynthesis, which occurs after mitochondrial damage, sends toxicity-limiting signals mainly to the cytosol during iron overload.

The comparison of Pink $1^{-/-}$with WT cells demonstrated five genotype-dependent effects: Strong excess inductions upon iron overload due to Pink1-ablation were observed for Fth1 (1.97-fold, $p=0.0291$ ), Ftl1 (1.64-fold, $p=0.0380)$, and Hmox1 (2.07-fold, $p=0.0562)$ mRNA, whereas deficient mRNA levels were observed for the ferritin superfamily member Rrm2 (0.48-fold, $p=0.0864)$ upon iron shortage. Moreover, in untreated cells, a reduction of basal Abce1 mRNA levels (0.70-fold, $p=0.0238$ ) existed in Pink1 $1^{-/}$cells a priori. Thus, the main impact of PINK1 on iron homeostasis factors occurred via the ferritin superfamily, which binds ferric ion $\left(\mathrm{Fe}^{+}\right)$. It is interesting to note that also the global proteome survey documented PINK1 impact on 8 iron homeostasis factors, among which FAC-downregulated MYL6, but also TCIRG1, and PCBP3 protein were implicated in the recruitment of soluble iron to $\mathrm{Fe}^{+}$-binding ferritin. It was previously observed that induction of mitophagy with stabilization of PINK1 protein occurred in parallel to iron liberation via ferritin reduction [98], so the stability of PINK1 versus ferritin appeared conversely regulated, and our finding of PINK1 deficiency to trigger ferritin mRNA upregulation is credible. A minor PINK1 impact targeted heme homeostasis via Hmox1 mRNA levels, as well as FECH, JMJD6, CYB5A, and GYPC protein abundance. Under untreated conditions, only the PINK1-dependent reduction of GLRX5 concerned the ISC biogenesis pathway, and this may relate to the reduction of basal mRNA levels for the ISC-containing Abce1.

\subsection{Quantitative Immunoblots for Validation and Mechanistic Analyses}

Now we wanted to assess in further validation experiments if the observed changes are also significant at the protein level upon analysis of additional MEF lines (Figure 5), focusing on three dysregulated factors whose transcript dysregulation had marked effect size (about two-fold up or down) and where a promising commercial antibody was available.

In the case of FTH1, a 9.72-fold $(p<0.0001)$ strongly significant induction after FAC was found in RIPA-extracted proteins from WT cells (Figure 5A) (in further SDS-extractions of sample pellets no specific immunoblot band was detected), but in contrast to the findings at mRNA level this FTH1 protein induction was significantly diminished in Pink1 ${ }^{-/-}$cells $(p=0.0476)$. Although this downregulation had not been significant in the mass spec analysis or the immunoblot validation for 3 mutant versus 3 
WT cells, the effect became clear when more WT cell lines were investigated. This finding is in good agreement with previous literature that could not document increased ferritin abundance in PD brains despite the iron accumulation [99-101]. As for Pink1 ${ }^{-1-}$ MEFs here, immortalized endometriotic cells were previously reported to respond to ever more excessive iron dosage with progressively lower ferritin protein levels and a converse increase of LC3A within ATG5/ATG7-dependent autophagy [102]. If ferritin-producing glia cells in a PARK6 brain cannot respond adequately to iron excess, then this weakness of antioxidant defenses might debilitate neurons and glia, leading to compensatory storage of iron overload in neuromelanin granules, and exhausting the storage capacity of neurons.

In the case of HMOX1 (Figure 5B), a strongly significant induction after FAC was found in WT cells (1.77-fold, $p<0.0001)$, and the analysis of Pink1 ${ }^{-/}$cells did not confirm a genotype-dependent increase at the protein level. Thus, the hypersensitive mRNA induction after FAC in Pink1 ${ }^{-l-}$ MEF might represent a compensatory effort to ensure sufficient protein abundance in a period of high turnover. Previous work reported the HMOX1 turnover to depend on ubiquitin/proteasome pathway inhibition by MG-132, and a PINK1 loss-of-function mutation decreased HMOX1 abundance in these experiments [103], so HMOX1 protein stability in Pink1 ${ }^{-/-}$cells after FAC might also be reduced upon analysis of more cell lines.

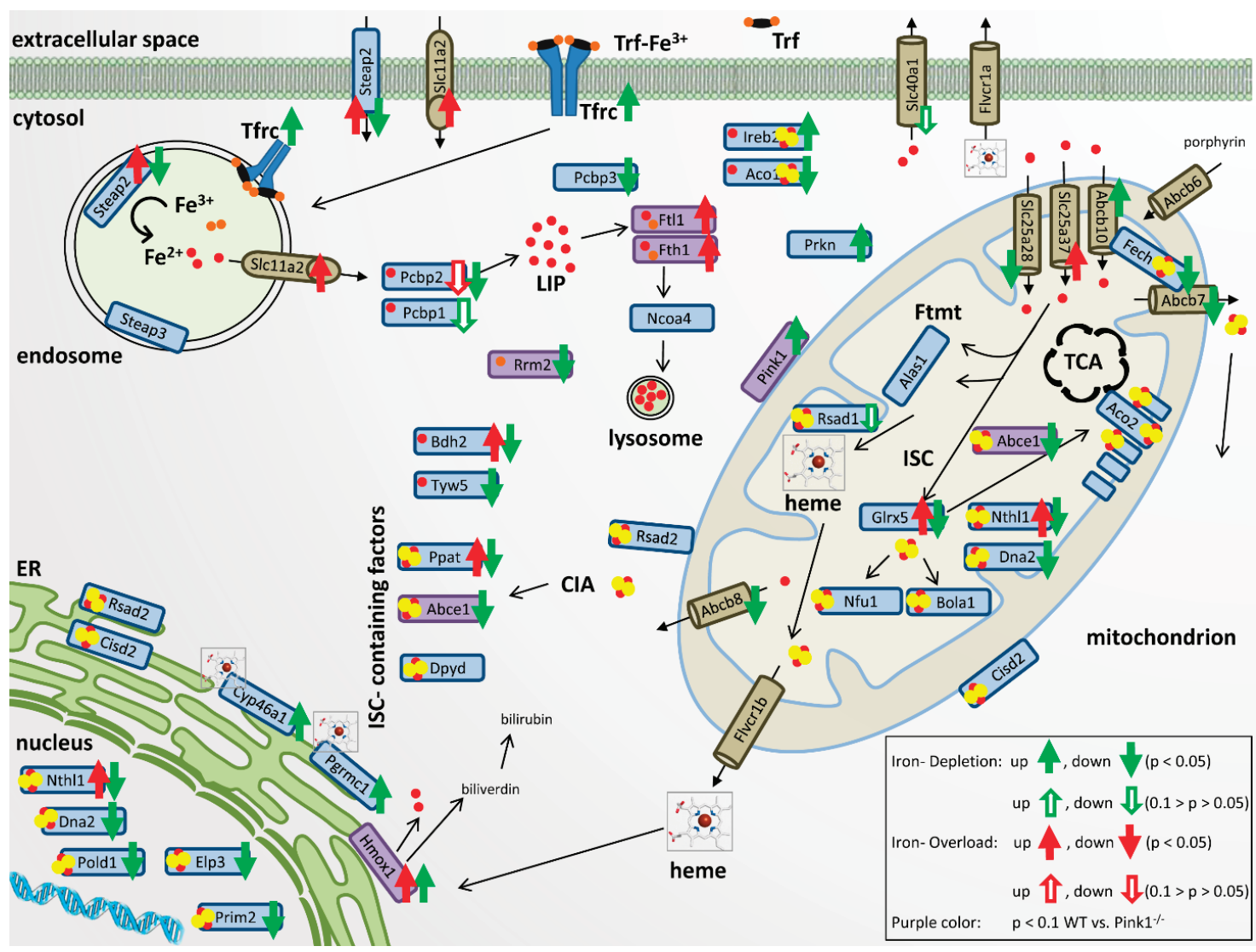

Figure 4. Overview of cellular iron uptake, storage, and export, iron transport and processing, mitochondrial iron homeostasis, heme production and turnover, iron-sulfur-cluster (ISC)-biogenesis and ISC-binding within the cells. For each factor with significant mRNA expression adaptation in room temperature (RT)-qPCR, green arrows indicate the direction of transcript change during iron depletion, while red arrows refer to changes during iron overload. Ferric iron $\left(\mathrm{Fe}^{+}\right)$is illustrated with orange dots and ferrous iron $\left(\mathrm{Fe}^{+}\right)$with red dots; ISC are represented by red and yellow dot clusters. CIA: cytosolic iron-sulfur-cluster assembly machinery; ER: endoplasmic reticulum; FTMT: mitochondrial ferritin; LIP: labile iron pool; TCA: tricarboxylic acid cycle. Factors with significant genotype-dependent changes with specificity for Pink1-/- cells are represented by purple coloring. 

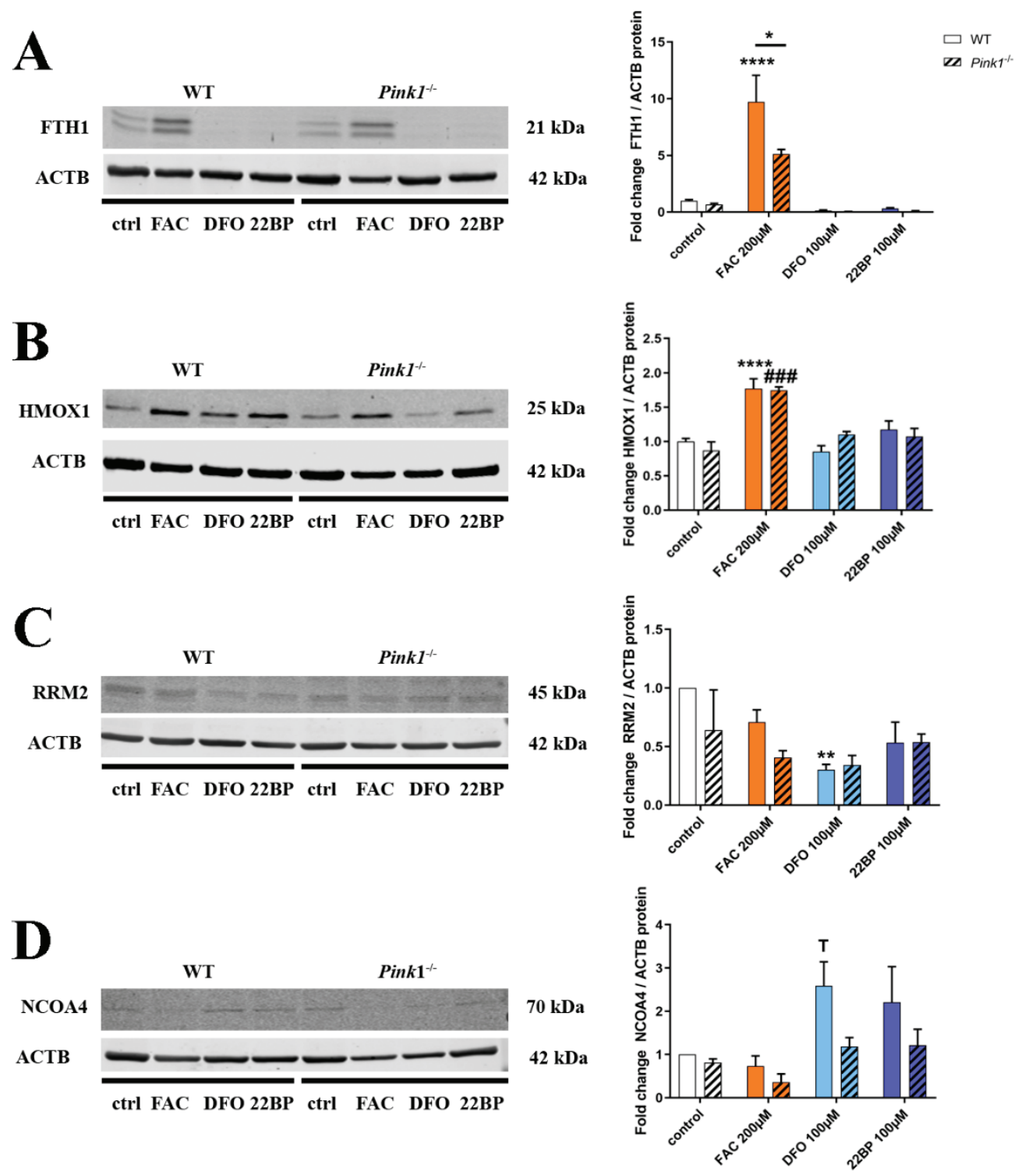

Figure 5. Quantitative immunoblots for (A) FTH1, (B) HMOX1, (C) Ribonucleotide reductase regulatory subunit M2 (RRM2), and (D) nuclear receptor coactivator 4 (NCOA4) in WT and Pink1 $1^{-1}$ MEF, under untreated control conditions (Ctrl), after iron overload (FAC) and after two different iron depletion drugs (DFO, 22BP), administered over $48 \mathrm{~h}$. Protein abundance signals were normalized to beta-Actin levels (Actin beta (ACTB)) as a loading control. The panels on the right show their densitometric quantifications, normalized to WT untreated conditions. WT $n=4-6$, Pink $1^{-/-} n=3$, one exemplary set is shown. The statistical trends or levels of significance are illustrated by symbols, namely T: $0.1>p>0.05,{ }^{*}: p<0.05,{ }^{* *}: p<0.01$, \#\#\#: $p<0.001,{ }^{* * * *}: p<0.0001$. Mutant cells are represented by dashed bars, WT cells by plain colors. Asterisks represent significance in WT MEF, treated versus untreated control, while hashtags refer to Pink1 ${ }^{-/-} \mathrm{MEF}$, treated versus untreated control. Genotype-dependent significant differences of Pink1-/- versus WT MEF are illustrated by horizontal lines below asterisks.

Regarding RRM2 (Figure 5C), a significant downregulation after DFO in WT cells was documented, in good agreement with proteome and transcript data.

To test mechanistically whether PINK1 as a master regulator of mitochondrial autophagy could also influence ferritin degradation via autophagy, quantitative immunoblots assessed the levels of the ferritinophagy adaptor protein NCOA4 (Figure 5D). As expected for the liberation of iron from reserve 
pools during iron-shortage, a 2.59-fold NCOA4 induction after DFO treatment was observed in WT cells, reaching a statistical trend despite strong clonal variability $(p=0.0593)$. In Pink1 ${ }^{-/}$cells this NCOA4 upregulation for ferritinophagy appeared diminished (to only 1.19-fold, $p=0.9432$ ), but certainly not augmented, and these effects did not reach significance upon analysis of $3 \mathrm{KO}$ versus 4 WT lines $(p=0.1445)$. Overall, these data in Figure 5D do not support the notion of an excessive ferritinophagy after FAC as an explanation for the abnormally low FTH1 protein upregulation in Pink1 ${ }^{-/}$cells (Figure 5A). We considered whether PINK1 phosphorylation might influence FTH1/FTL1/HMOX1 protein stability independent of autophagy/proteasome degradation, similar to its activating/stabilizing influence on Ubiquitin and Parkin [104]. To first ensure a deeper understanding of the mechanisms governing iron regulation and their modification by PINK1, we analyzed the data further to understand the adaptations of relevant nuclear transcription factors, and the responses of post-transcriptional control by IRP1/IRP2 over ferritin mRNA translation repression.

\subsection{Regulation of Relevant Transcription Factors with Their Targets Is Subtly Modified by PINK1}

To define the relevant nuclear events governing the transcriptional responses, those transcription factors that had shown abnormal abundance in the proteome profile, and other relevant transcription factors-both, for the antioxidant response to FAC and the hypoxic response to iron chelators-were assessed also in their rapid mRNA regulation by the highly quantitative RT-qPCR method (Figure 6A, Supplementary Figure S6), together with their best-established transcription targets.

As a coordinator of hypoxic transcription efforts, HIF1A activity was previously found to be stabilized during PINK1 deficiency [105], suggesting a negative correlation between both factors. As coordinator of antioxidant transcription efforts, NRF2 (encoded by the Nfe2l2 gene) activity was previously reported to enhance Pink1 expression, and to accumulate and translocate to the nucleus when PINK1 induced and stabilized [106,107], suggesting a positive correlation between these two factors. NRF2 is known to enhance the transcription of Fth1/Ftl1/Hmox1 during ferroptosis [108,109], so a hypothetical NRF2 compensatory hyperactivity upon PINK1 absence might explain excessive transcription inductions after FAC. The NRF2 inhibitor KEAP1 is degraded by autophagy [110], therefore altered autophagy due to PINK1 absence might feedback onto NRF2 activity.

Indeed, for transcription factor TFEB, as a coordinator of autophago-lysosomal pathway activity (Supplementary Figure S6A), in untreated Pink1 ${ }^{-/}$cells a significant elevation of mRNA levels suggested augmented activity of general autophagy, perhaps in compensation of the selective mitophagy deficit. During iron depletion, WT cells efficiently induced Tfeb mRNA, while Pink1 ${ }^{-/}$cells were unable (Supplementary Figure S6A). However, these changes appeared to only have subtle impact, given that downstream TFEB targets Ctsd/Ctsf/Sqstm1 did not exhibit significant genotype-dependent expression effects. Transcription factor MITF as an additional coordinator of autophago-lysosomal activity (Supplementary Figure S6B) showed a consistently stronger expression induction after FAC, but Mitf mRNA and its downstream target $C t s b$ exhibited no genotype-dependent expression adaptations.

NRF2 encoding Nfe2l2 mRNA levels showed no regulation at all (Figure 6A), but its inhibitor Keap1 showed a trend towards reduction after 22BP, in good agreement with its protein levels in Figure 2B and Supplementary Figure S2B. Among the downstream targets of NRF2/KEAP1, several factors like Gpx1/Gpx3 showed no upregulation after FAC, other factors like Nqo1/Prdx1 showed induction after FAC and reduction after $\mathrm{DFO} / 22 \mathrm{BP}$ without any genotype-dependent modulation, and only Fth1/Ftl1/Hmox1 showed the FAC-triggered hypersensitive induction in Pink1 ${ }^{-1-}$ cells, so NRF2 alone does not explain the response pattern.

JUND (Jun proto-oncogene) is a component of antioxidant NRF2 transcription factor complexes [111], but did not exhibit induced expression after FAC, instead, it showed strong consistent mRNA induction after iron chelation (Supplementary Figure S6C), consistent with the upregulation of JUND protein after 22BP in Supplementary Figure S2B. Its downstream target Mmp14 in Pink1 ${ }^{-/}$cells showed a trend to stronger reduction after 22BP, in good agreement with the downregulated MMP14 
protein abundance after 22BP in Supplementary Figure S2B, as a converse regulation to MMP14 protein upregulation after FAC in Figure 2B.

Hifla mRNA as coordinator of hypoxic transcription responses showed no relevant expression regulation (Supplementary Figure S6D), despite the upregulation of HIF1A protein after 22BP in Figure 2B and Supplementary Figure S2B. Its upstream regulator EGLN1 (Egl-9 family hypoxia-inducible factor 1) [112] showed strong and consistent expression inductions after iron chelation, in agreement with EGLN1 protein upregulation in Figure 2B and Supplementary Figure S2B. For their downstream targets Hk1 (Hexokinase 1)/P4ha2 (Prolyl 4-hydroxylase subunit alpha 2)/Nos2 (Nitric oxide synthase 2 ), iron shortage triggered clear mRNA inductions as the basis of their increased protein abundance in Figure 2B and Supplementary Figure S2B. Overall, despite the very clear activation of this hypoxia pathway after iron deprivation, there were no PINK1-dependent effects in this pathway whatsoever. The upregulation of FOXO3 protein after 22BP in Figure 2B was also clearly reproduced at the mRNA level (Supplementary Figure S6E), but interestingly this expression induction was significantly diminished in Pink1 $1^{-/}$cells. Again the impact on downstream targets like Bnip3 (BCL2 interacting protein 3)/Gabarapl1 (GABA type A receptor-associated protein-like 1) was too subtle to trigger genotype-dependent effects there.

Regarding the hypoxic transcription factor MEF2D (Myocyte enhancer factor 2D) and its upstream splicing modulator RBFOX2, the expression modulations were small, but in the case of their downstream targets Homer1/Jmjd6/Rrm2, the expression modulations by iron deprivations were strong and consistent. PINK1-deficiency was found in Supplementary Figure S2E to trigger RBFOX2 protein upregulation, and indeed genotype-dependent effects were observed among the mRNA targets Jmjd6 (another splice modulator) and Rrm2, in both cases with a trend towards reduced transcript levels in Pink1 ${ }^{-1-}$ cells (Supplementary Figure S6F).

Overall, iron modulates the expression regulation of transcription factors and their mRNA targets in good agreement with global proteome profiles, particularly for the hypoxia pathway after iron chelator treatment. In contrast, the PINK1-dependent effects on transcriptional control were only subtle, with a consistent change in several downstream targets being documented only for the hyper-reactivity of Fth1/Ftl1/Hmox1 in the antioxidant NRF2/KEAP1 pathway, while other NRF2 targets did not show this regulation pattern.

\subsection{Regulation of the Post-Transcriptional Control over Iron Homeostasis Is Modified by PINK1}

Global proteome and RT-qPCR data were then re-examined in order to assess, to what degree post-transcriptional control mechanisms play a role and are modulated by PINK1 (Figure 6B). The activities of iron-regulatory proteins IRP1/ACO1 and IRP2/IREB2 depend on the stability of their [4Fe-4S] clusters, which are generated in mitochondria from [2Fe-2S] clusters that bind to GLRX5, to then be processed further [113]. When the ISC are not available for IRP1/IRP2 or are damaged, the mRNA for Tfrc will be stabilized to increase iron import, while repression of mRNA translation will occur for ferritin heavy and light subunits to maximize iron liberation [95]. Some repair of IRP1 can occur via the [2Fe-2S] clusters of CISD1/CISD2, which protect the cells from ferroptosis [114,115].

Iron overload triggered a stronger Glr $x 5$ mRNA induction in Pink1 $1^{-/-}$cells, possibly as a compensatory effort for the significant decrease of GLRX5 protein already at untreated condition as well as after FAC in Pink1-/- cells, while iron shortage reduced GLRX5 at mRNA and protein level in all cells (Figure 6A,B).

Also for Aco1/Irp1, iron overload triggered a stronger RNA induction in Pink1 $1^{-/}$cells, while iron shortage again reduced Aco1 at mRNA level in all cells, in both conditions these transcript regulations maintained normal protein levels.

FAC treatment had no impact on IRP2 mRNA Ireb2, which is rapidly degraded under normal iron concentrations so that no IRP2 protein was detectable. 22BP treatment resulted in marked stabilization of Ireb2 mRNA, and more than 2-fold upregulation of the protein, as previously shown. 


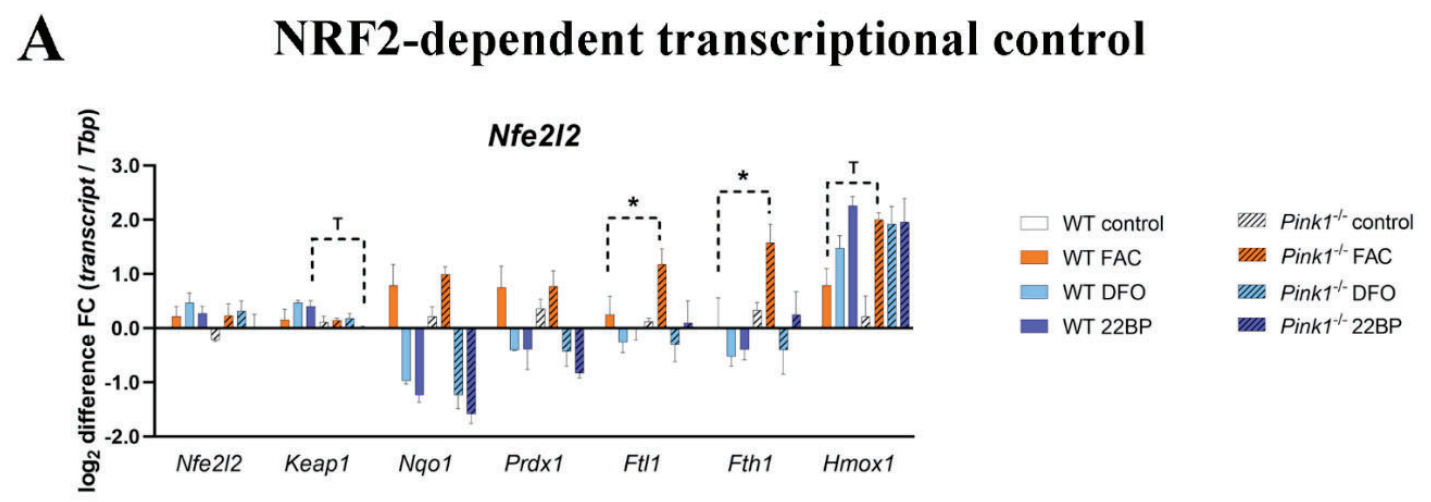

B

Post-transcriptional control
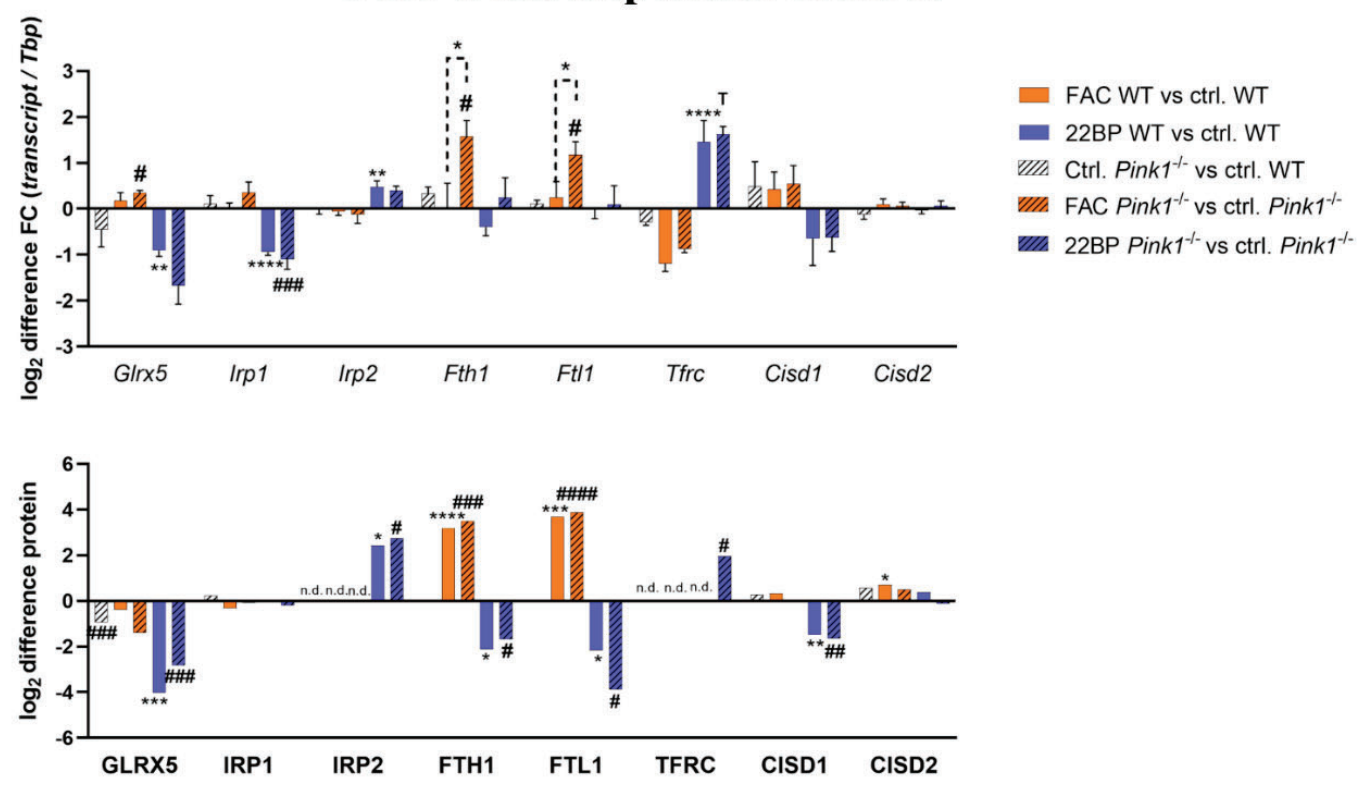

Figure 6. Adaptation to changing iron concentrations for key factors in (A) the Nuclear factor, erythroid 2 like 2, Nfe212 (NRF2) transcription factor complex with its modulator Kelch-like ECH associated protein 1 (KEAP1) and their downstream transcript targets, as well as (B) the post-transcriptional control of iron import and storage. For mRNA expression analysis, the Tbp levels were used as loading control and after normalizing the fold changes to control conditions in WT MEF, the $\log _{2}$ fold changes of individual fold changes were calculated. The fold change of protein abundance label free quantification (LFQ) value was also represented as $\log _{2}$ difference. Mutant cells are represented by dashed bars, WT cells by plain colors. Asterisks represent significance in WT MEF, treated versus untreated control, while hashtags refer to Pink $1^{-/-}$MEF, treated versus untreated control. To simplify the overview, in (A) only genotype-dependent significances are shown, whereas (B) includes all significances with respect to control conditions. Detailed $p$-values and individual fold changes can be seen in Supplementary Tables S1,2. The statistical trends or levels of significance are illustrated by symbols T: $0.1>p>0.05,{ }^{*}$ or \#: $p<0.05,{ }^{* *}$ or \#\#: $p<0.01,{ }^{* * *}$ or \#\#\#: $p<0.001,{ }^{* * * *}$ or \#\#\#: $p<0.0001$.

For the ferritin heavy and light chain, after FAC the mRNA induction was hyper-reactive in Pink $1^{-/}$cells as described previously, while the protein levels appeared similarly upregulated during iron overload, and similarly downregulated after iron deprivation, in this analysis of only 3 WT versus 3 mutant MEF lines by mass spectrometry.

The transferrin receptor exhibited mRNA downregulation under iron excess and upregulation under iron shortage, its protein abundance was detectable and high only in Pink1 ${ }^{-/}$cells during 
iron shortage, as a possible correlate of an increased iron import need of cells affected by Parkinsonian pathogenesis.

Regarding the IRP1-repair factors CISD1/CISD2, after FAC an upregulation for CISD2 protein was detected, whereas CISD1 protein was downregulated after 22BP. A clear genotype-dependent effect was not observed. The mRNAs of Cisd1 and Cisd 2 did not show significant changes, although Cisd1 levels appeared lower during iron shortage.

\subsection{PINK1-Dependent Effects on Expression in Human Skin Fibroblasts}

To test whether the main effects observed in MEF can be reproduced also in human adult skin fibroblasts, we used cells from 7 healthy individuals versus 3 PARK6 patients with G309D-PINK1 mutation (experiment design in Figure 7A) that were previously shown to exhibit (i) lipid peroxidation, (ii) apoptotic vulnerability, (iii) expression dysregulation of the ferrireductase alpha-synuclein and the kinase LRRK2 (responsible for the PARK1/4 and PARK8 variants of PD, respectively), and (iv) abnormal mitoribosomal translation due to ABCE1 function impairment [14,17,116-123].

In good agreement with the mouse data, the analysis of iron dosage impact on mRNA expression levels by RT-qPCR demonstrated $A B C E 1$ transcripts significantly and consistently reduced after $\mathrm{DFO} / 22 \mathrm{BP}$ in WT and mutant cells (see Figure 3C), whereas they were induced after FAC selectively in mutant cells (Figure 7B). This genotype-dependent upregulation again may be because ABCE1 protein contains an iron-sulfur-cluster and its instability upon oxidative/nitrosative stress has to be compensated by antioxidant compensatory efforts in the patient cells.

The converse regulation was observed for TFRC mRNA, where the expected negative correlation with iron led to a consistent increase after DFO/22BP versus a decrease after FAC.

In the case of FTH1 mRNA, the PINK1-dysfunction triggered a significant excess induction (Figure 7B), validating our analogous observation in MEFs. It is noteworthy that the FTH1 mRNA showed a trend to increased levels already in the control medium (which has maximized non-toxic iron levels) in cells from adult PARK6 patients, a feature that was not detected in embryonic fibroblasts from mice. For FTL1 mRNA, generally increased levels were observed in PARK6 cells, but without significance due to high variation among the mutant cell lines. The ferritin superfamily member RRM2 mRNA exhibited a significant decrease after 22BP, as in mouse cells. It also showed significant genotype-dependent regulations in control medium and after FAC, both levels being decreased for mutant cells, in contrast to FTH1. Thus, also in the human organism PINK1 preferentially impacts the NRF2-dependent antioxidative response to iron overload.

There were no genotype-dependent effects for the other factors studied, but iron deprivation tended to upregulate FOXO3 mRNA levels as observed in mouse, while significantly and consistently downregulating KEAP1 and TFEB mRNA (Figure 7B), two observations that are opposite to the upregulations observed in embryonic mouse cells (Figure 6A). Age-dependent changes in the nuclear transcription regulation of autophagy may help to explain this contrast for both factors. 


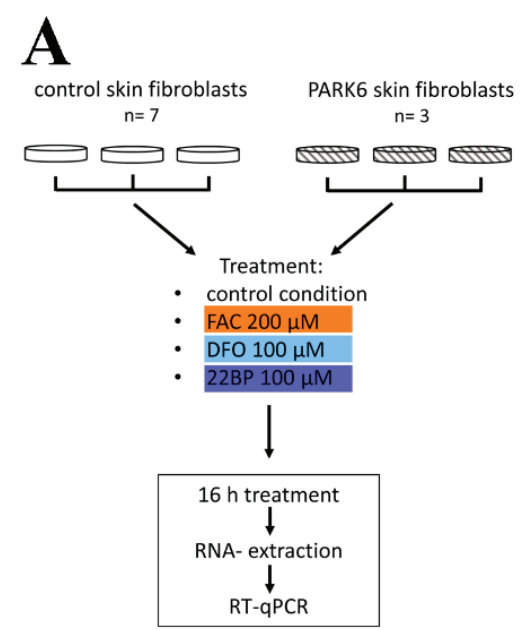

\section{B}
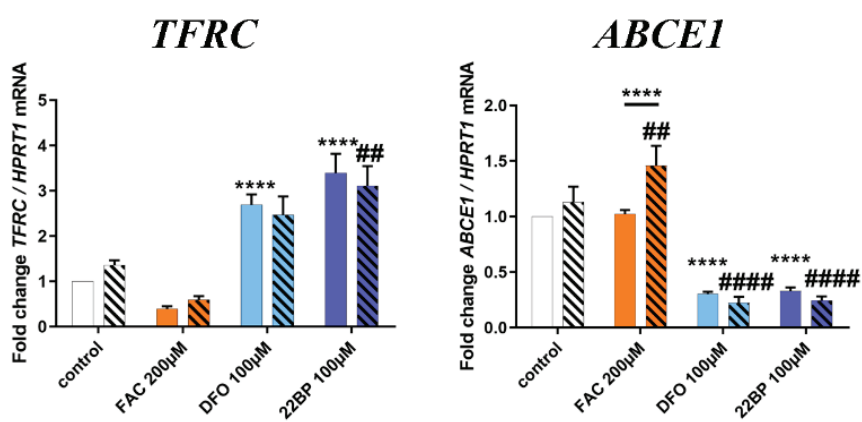

$\square$ CTRL
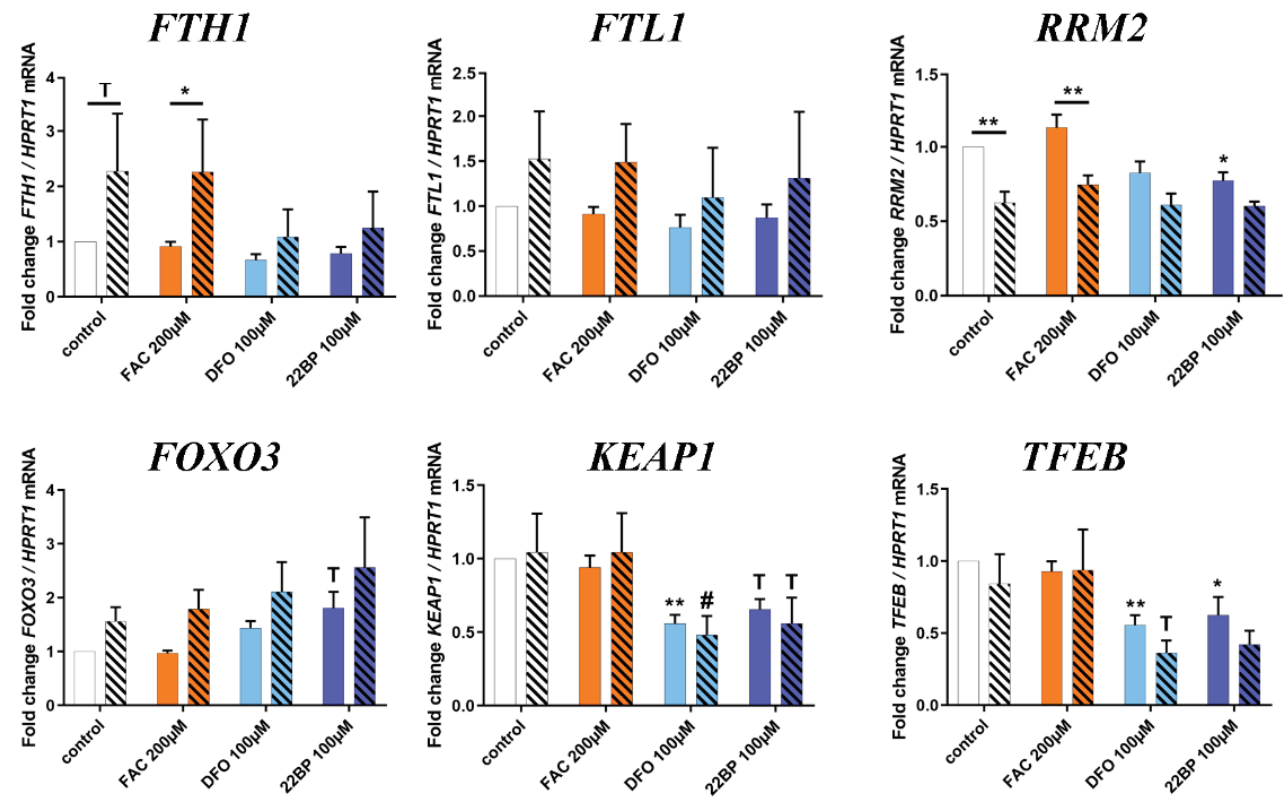

Figure 7. (A) Schematic representation of the experimental setup. Human fibroblasts of healthy controls $(n=7)$ and PARK6 patients $(n=3)$ were either left untreated or incubated with FAC, DFO, and 22BP for $16 \mathrm{~h}$, and the extracted RNA was analyzed by RT-qPCR. (B) Changes of mRNA expression for key iron homeostasis factors, and crucial downstream effects, which had been significantly dysregulated in previous mouse experiments. Expression levels were normalized against HPRT1 mRNA as a loading control. Mean values with SEM are shown, normalized to the untreated control condition. The statistical trends or levels of significance are illustrated by symbols T: $0.1>p>0.05$, ${ }^{*}$ or \#: $p<0.05$, ** or \#\#: $p<0.01,{ }^{* * * *}$ or \#\#\#: $p<0.0001$. Asterisks represent significant changes in control fibroblasts, treated versus untreated control, while hashtags refer to PARK6 patient cells, treated versus untreated. Genotype-dependent significant differences between patients and controls are illustrated by horizontal lines below asterisks.

\section{Discussion}

Our data represent a systematic pioneer effort to document physiological regulations of the global proteome and transcript levels for most factors with relevance to iron, ISC, and heme homeostasis, upon acute changes in iron availability. The fibroblast data were also used to explore molecular mechanisms, how PINK1 mutations trigger the iron brain accumulation that is seen in PD. The global proteome profiles confirmed the well-known converse regulation of both ferritin chain subunits after 22BP versus FAC, and newly identified a converse regulation for the iron-binding purine degradation 
enzyme $\mathrm{XDH}$, the mitochondrial lipid transporter CPT1A, the collagen degrading factor MMP14, and the glycogen mobilizing enzyme PYGL (only the latter showed an indirect correlation with iron availability). In contrast, the heme oxygenase HMOX1, the nuclear transcription modulator CREG1, and the ferritin superfamily member and nucleotide synthesis enzyme RRM2 showed optimized abundance at untreated conditions, while 22BP more than FAC treatment both triggered upregulation of HMOX1 and CREG1 protein, versus downregulation of RRM2 protein. The targeted mRNA quantification studies of mouse and human fibroblasts showed very good reproducibility, regarding the induction of both ferritin subunits by FAC at the transcriptional level, which exacerbates upon the presence of PINK1 mutations. With high consistency between mouse and human fibroblasts, iron deprivation decreased the expression of $A B C E 1$ and $R R M 2$ mRNA, as components of nucleotide synthesis/surveillance pathways.

Iron chelators trigger a transcriptional induction of the mitophagy-mediators Pink1 and Prkn in MEF, according to our novel observations. This is an important finding to explain the previous reports in C. elegans that mitophagy is induced upon iron shortage [11,12]. A similar induction of Pink1 and Prkn, accompanied by increased autophago-lysosomal degradation of mitochondria, was also reported in human neuroblastoma cells upon deprivation from fetal calf serum, which contains transferrin as the key supplier of iron during cell culture [19]. Interestingly, iron shortage also leads to a consistent upregulation of beta-synuclein protein (SNCB), an antagonist of alpha-synuclein. While SNCB was shown to have some ferrioxidase activity, alpha-synuclein is known as a ferrireductase [124-127], similar to STEAP3 [128], and has a physiological localization at vesicles/endosomes and at the interface between mitochondria and endoplasmic reticulum [129]. In the context of human Parkinson pathogenesis, it is noteworthy that the main driver of neurodegeneration in PD, namely the excessive dosage and aggregation of the protein alpha-synuclein, can be modulated by iron via direct binding to an IRE in its mRNA $5^{\prime}$ UTR as well as direct binding to the encoded protein $[130,131]$. Although the upregulation of $\mathrm{SNCB}$ after iron deprivation raises the question if its antagonist SNCA is upregulated after iron excess and perhaps influenced by PINK1 similar as Fth1/Ftl1 mRNA at their 5'UTR hairpins via IRP1/IRP2, it is not possible to investigate the connections between iron and alpha-synuclein further in MEF due to its low abundance in these cells. Experiments in primary neuron cultures will be necessary. However, it is noteworthy that a global transcriptome survey via oligonucleotide microarrays detected an 8-fold induction of alpha-synuclein transcripts in human fibroblasts from PD patients with Pink1 mutation [119]. Certainly, the observation of increased lipid peroxidation in these PARK6 patient fibroblasts suggests that an enhanced vulnerability for ferroptosis may be present in these cells [17]. Thus, both the negative correlation of iron with Pink1/Prkn expression and its positive correlation with alpha-synuclein aggregation may contribute to its toxicity, modulating mitophagy, and the neurodegenerative process in PD.

Iron deprivation conversely downregulated RRM2, an effect that will lead to reduced dNTP availability for mtDNA repair, and at the same time to increased superoxide release from uncoupled mitochondria as a cause for mtDNA damage, according to two previous studies [132,133]. This is highly important, given that excessive damage to mitochondrial DNA in the PD brain tissue is a well-established finding [134]. The global proteome profiles, illustrated in Figure 2 and Supplementary Figures S1-S4, and the transcript quantification of iron-binding factors, illustrated in Figures 3 and 4, documented that decreased abundance and expression of nucleotide synthesis/surveillance factors after iron deprivation is a widespread feature, which has to be taken into account when iron chelators will be tested for the neuroprotective therapy of PD.

With consistency between WT and mutant cells, iron shortage upregulated the plasma membrane iron import factor Tfrc mRNA together with Ireb2 as a stabilizer of its mRNA [135]. Iron depletion also upregulated the mitochondrial membrane iron import factor $A b c b 10$. In contrast to this avid activation of iron recruitment proteins upon iron deficiency, the expression of intracellular iron disposal factors was in a positive correlation with iron availability. This iron-dependent expression was particularly strong for the endosomal factors STEAP2 and DMT1 (encoded by Slc11a2), and exceptionally significant for Ppat, 
Nthl1, Dna2, Pold1, Tyw5, and slightly for Prim2. All of these factors contain ISC, and importantly, they act as components of RNA/DNA surveillance. NTHL1 is a DNA N-glycosylase that is mainly localized in the nucleus but also found in mitochondria [136]. It catalyzes the first step in base excision repair and binds a [4Fe-4S] cluster [9]. Nthl1 mRNA seemed to react very sensitively with a reduction to almost $50 \%$ upon iron depletion and induction to $135 \%$ after iron overload. PPAT belongs to the purine/pyrimidine phosphoribosyltransferase family and catalyzes the first step of de novo purine nucleotide biosynthetic pathway. It also possesses a [4Fe-4S] cluster, which is needed for protein maturation [9]. PPAT maturation and subsequent function are affected in the neurodegenerative disorder Friedreich Ataxia. This disease is caused by loss-of-function mutations in the Fxn gene encoding the frataxin protein, which starts the ISC biogenesis within mitochondria $[137,138]$. Thus, the transcript reductions of Nthl1 and Ppat probably reflect limited ISC supply from mitochondria that causes impaired maturation/stability of both proteins [139].

All other factors with a downregulated expression upon iron deficiency are either involved in the complex synthesis of ISC or contain one or several ISC. Among them were Bola1, Nfu1, and Glrx5, which play roles in the synthesis of ISC and are localized inside mitochondria. The cytosolic tRNA modification factor TYW5 associates with iron and was also clearly reduced after iron deprivation. A downregulation was noted for ISC-containing cytosolic IRP1/Aco1 as well as for ISC-containing Rsad1 (in PINK1-deficient cells), which encodes a heme chaperone in the mitochondrial matrix [140]. However, ISC-containing FECH in mitochondria did not show relevant expression dysregulation. This suggests that ISC homeostasis is not entirely disrupted upon depletion of extracellular iron or in the absence of Pink1, but instead, specific vulnerabilities exist, in particular for RNA/DNA quality and genome stability through Ppat/Nthl1/Dna2/Prim2/Pold1 deficiency. This is a central finding, since DNA integrity is crucial for the healthy lifespan [141], and since the DNA repair pathway was recently identified as the most important modifier of onset age and progression velocity in neurodegenerative diseases [142-144]. Jointly, all these data underline the importance of iron for the RNA/DNA quality surveillance, inside and outside mitochondria.

Further mRNA inductions in response to iron deprivation included Ireb2/IRP2 as a stabilizer of Tfrc mRNA [135], the more than 2-fold induction of the heme-release factor Pgrmc1 [145], and a massive 4- to 5-fold induction of $\operatorname{Hmox} 1$ (the rate-limiting enzyme in heme-degradation) in response to 22BP, as another possible pathway of iron recruitment. The previously observed impaired Hmox1 induction after oxidative stress damage in Pink1-depleted cells [103] was not detected in MEF in the absence of stress factors. Iron depletion probably increases HMOX1 via HIF1a induction (a well known response to iron depletion) and FAC causes oxidative stress, probably leading to Nrf2 induction and an increase in HMOX1 via this pathway. It is interesting to note that iron-starved cells trigger strong induction of the mitochondrial ferrochelatase-associated heme-release factor Pgrmc1, but not a similarly strong effort to export ISC from mitochondria through $A b c b 7$ induction. The novel observation that iron depletion induces Cyp46a1 as the rate-limiting enzyme of cholesterol degradation provides a hint of why iron-deficiency anemia patients show lower blood cholesterol levels, and how iron influences steroidogenesis [146,147].

Current knowledge proposes iron deficiency or hypoxia to act via nuclear HIF-1/FIF-2a/HIF-b, triggering a transcriptional induction of Tfrc together with Hmox1, Slc11a2 (DMT1), Slc40a1 (FPN1), $E p o$, and $C p$ [72]. However, in acutely iron deprived MEF, we observed a strong upregulation only for Tfrc and Hmox1, which contrasted with mild downregulation for Slc11a2 and Slc40a1.

Under untreated conditions, significant dysregulation with Pink1 ${ }^{-/}$genotype-dependence was documented for the ISC-biogenesis factor GLRX5 and for the mRNA of ISC-associated ABCE1 (Supplementary Figure S2E, Figures 3 and 4, also after 22BP in Figure 2B). ABCE1 is one of the most conserved proteins in evolution and is expressed in all organisms except eubacteria. Because of its fundamental role in translation initiation, ribosome biosynthesis and/or ribosome recycling, ABCE1 is essential for life. The reduced ABCE1 function in PARK6 patient fibroblasts was already documented by our previous work to impair the ribosomal translation of mitochondrial precursor proteins, triggering 
widespread dysfunction for most mitochondrial pathways [122,124]. Our novel mammalian data confirm that $\mathrm{ABCE1}$ has a unique link to the mitophagy factor PINK1, as was observed previously also in D. melanogaster. These experiments in flies demonstrated that nucleus-derived mRNAs encoding mitochondrial precursor proteins, such as the complex-I $30 \mathrm{kD}$ subunit continuously translated at the outer mitochondrial membrane surface, may be damaged and stall the ribosomal translation machinery during stress periods. This leads to a toxic C-terminal extension of certain amino acids non-coded by mRNA template. In a PINK1-dependent manner, this recruits co-translational quality control factors for RNA/proteins in a process named MISTERMINATE, and triggers mitophagy. During this mitochondrial surveillance process, NOT4 (Ccr4-Not transcription complex subunit 4) generates poly-ubiquitin signals on the co-translational control protein $\mathrm{ABCE1}$, thus attracting autophagy receptors to the mitochondrial outer membrane and contributing to mitophagy initiation $[122,124,148]$. Thus, fly mitophagy is regulated together with proteostasis in PINK1-dependence via ABCE1. It is relevant to note that longevity is increased upon several genetic perturbations of mRNA translation within the mTOR pathway (Mechanistic target of rapamycin kinase) in yeast, C. elegans, and D. melanogaster and by mutations that slow down the expenditure of cellular energy by ribosome biogenesis $[149,150]$. Therefore, it will be interesting to assess the lifespan effects of different ABCE1 mutations in the future.

Strong homozygous depletion of the ABCE1 homolog pixie in D. melanogaster results in early lethality [151]. In addition, the homozygous mouse knockout of Abce1 is embryonically lethal, according to the International Mouse Phenotyping Consortium (IMPC) (https://www.mousephenotype. org/data/genes/MGI:1195458), the international mouse phenotyping consortium. As an ATPase (Adenosintriphosphatase), ABCE1 is responsible for the splitting of the two ribosomal subunits and is thus important for translation termination in mammalian cells, yeast and archaea. To fulfill these roles, it harbors two essential diamagnetic $[4 \mathrm{Fe}-4 \mathrm{~S}]^{2+}$ clusters [152]. The depletion of Abce1 was reported to induce the accumulation of ribosome-associated isolated mRNA-3'-UTRs, consistent with a model of ribosome stalling [153]. It is also known as RNaseL-inhibitor and exerts selective control over the stability of mitochondrial mRNAs during interferon-alpha responses to infection [154]. Decreased ABCE1 protein levels were caused by the induction of ROS and this was attributed to the chelation of iron with subsequent loss of stability of ABCE1, indeed its yeast ortholog Rli1 was reported to be crucial for the growth suppression by ROS $[93,153]$. Interestingly, Abce1, as well as Hbs1l (Hbs1 like translational GTPase) transcript levels, were shown to be upregulated in the brains of PD patients [148]. $\mathrm{Hbs} 1 \mathrm{~L}$ is a member of the GTP-binding elongation factor family and was reported to be involved in the regulation of fetal hemoglobin levels [155]. These observations are in accordance with the increased transcript levels of $A B C E 1$ after iron overload in human PARK6 fibroblasts (Figure 7).

Selectively after iron overload, which is known to trigger mitochondrial reprogramming and oxidative/nitrosative stress [156], Pink1-ablation was observed to induce expression upregulations of several cytosolic iron homeostasis factors. A genotype-dependent trend towards upregulation was observed for Hmox 1 as a factor that protects against heme cytotoxicity. Crucial significant and consistent upregulations appeared after FAC treatment in Pink1-ablated cells for Fth1 and Ftl1, the two subunits of the ferritin chain $[157,158]$. The hyper-responsive induction of Fth1/Ftl1/Hmox1 in Pink1 ${ }^{-/}$cells was mirrored by a similar pattern for the ISC-pathway member Glrx 5 and for the iron-regulatory Aco1 mRNA, perhaps pointing to joint regulation within the same stress response pathway. These FAC-triggered hyper-reactive transcriptional responses of $F t h 1 / F t l 1 / H m o x 1$ have NRF2 in common as a transcription factor, but other transcript targets of NRF2 that are not involved in iron homeostasis do not show this regulation pattern, so additional mechanisms must be involved. Despite this transcriptional induction, the ferritin chain heavy subunit protein was significantly decreased in Pink1-ablated cells upon immunoblot studies. The repression of Fth1 and Ftl1 mRNA translation is a well-established regulation with a physiological role during iron shortage, mainly mediated by the incorporation of mitochondria-generated ISC into the IRP1 and IRP2 proteins. When the cells are iron deprived and ISC cannot be produced, then IRP1 and IRP2 will repress ferritin biosynthesis post-transcriptionally. It is conceivable that this repression occurs erroneously also when mitochondria are dysfunctional and 
unable to provide ISC. Although the global proteome profile of MEF quantified less than 4000 out of 80,000-400,000 existing cellular proteins, and more exhaustive studies should be done on all proteins of the mitochondrial fraction, our data show several preliminary hints that ISC unavailability/instability due to mitochondrial dysfunction in Pink $1^{-/-}$cells might explain the abnormal regulation of iron homeostasis. Firstly, the cytosolic ISC-repair factor CISD2 increased after FAC (Figure 2A), suggesting an instability of ISC-proteins under these conditions. It is relevant to note that the genetic ablation of CISD1 in mouse causes neurodegeneration with Parkinsonian phenotypes [159], and that iron shortage in MEF cells triggered CISD1 deficiency. Secondly, Aco1 mRNA encoding IRP1 appeared induced in Pink $1^{-/}$cells after FAC treatment (Supplementary Figure S5), thus maintaining the steady-state protein abundance normal, but possibly the ratio between the ISC-containing ACO1 holoenzyme versus the ISC-deficient IRP1 apoenzyme shifted. Thirdly, the selective upregulation of TFRC protein in Pink1 ${ }^{-/}$cells after 22BP, together with the hyper-responsive induction of the mitochondrial iron transporter Slc25a37 (Mitoferrin-1) after FAC in Pink1 ${ }^{-/-}$cells (Supplementary Figure S6), might both represent a compensatory effort to maximize iron import because of a mitochondrial impairment of iron utilization, e.g., in the ISC and heme biosynthesis pathways. Fourth, the mitochondrial ISC-biosynthesis factor GLRX5 was decreased to 0.52-fold in absence of treatment in Pink1 ${ }^{-1-}$ cells, and to 0.77-fold after FAC in WT cells (Supplementary Figure S2E). In human non-hematopoietic cells with GLRX5 deficiency, the reported consequences include a deficit of mitochondrial ISC biogenesis, increased IRP1-mediated repression of ferritin mRNA translation, and elevated TFR1-mediated iron import with subsequent accumulation of cytosolic/mitochondrial iron [57]. It was also observed that cells with GLRX5 deficiency show abnormally high LIP, repressed ferritin levels, and an augmented vulnerability to ferroptosis [160], as appears to be the case also for Pink1 ${ }^{-/}$cells. Notions that the mitochondrial dysfunction inherent in PD leads to progressively conspicuous efforts to maximize ISC-biogenesis are also supported by a report that the abundance of the ISC export factor ABCB7 increased abnormally over time in the neurotoxic MPTP mouse model of PD [161]. The concept that this mitochondrial dysfunction in PD leads to abnormal post-transcriptional regulation of iron homeostasis via IRP1 has also been confirmed in previous studies that showed the ferritin mRNA translation repression to be caused by sustained IRP1 activity in PD rodent models as well as patient brains [162,163], and that respiratory dysfunction due to complex I inhibition triggers decreases ISC-biogenesis and induces IRP1 activity [164]. These observations are similar to our data on Pink1 ${ }^{-/-}$MEF, as summarized in the Graphical Abstract.

A previous mass spectrometry study in WT rat primary cortical neurons treated with FAC over $24 \mathrm{~h}$ showed a 10-fold increase of ferritin protein [54], in good agreement with our findings in WT cells after $24 \mathrm{~h}$. Such induction of ferritin and Hmox 1 has a cytoprotective effect, as previously shown [165,166], but is not maintained in PD brains despite the iron accumulation [99,101]. Inadequate ferritin responses to iron excess would lead to an accumulation of the LIP and mitochondrial iron, a phenomenon that was demonstrated in PD brain tissue by recent studies that employed Mössbauer spectroscopy $[167,168]$. This scenario is compatible with our proteome findings that MYL6 showed a massive downregulation after FAC selectively in Pink $1^{-1-}$ cells, and that PCBP3 exhibited decreased levels without treatment in Pink1 $1^{--}$cells since both factors are involved in iron-chaperone functions to deliver components of the LIP to ferritin $[76,169,170]$.

The abnormally high LIP will bind to the protein Pirin and thus modulate innate immunity via the NFkB pathway (Nuclear Factor "kappa-light-chain-enhancer" of activated B-cells) [109], in good agreement with our observations in the proteome profile that numerous inflammatory factors are dysregulated, and with previous reports that PINK1/PARKIN dysfunction modulates NFkB, causes neuroinflammation, and impairs defenses against invading bacteria $[35,38,40,171]$. Under iron-saturated conditions, Aco1-encoded IRP1 protein functions as aconitase to prevent citrate accumulation in the cytosol, thus harmonizing fatty-acid synthesis and protein acetylation with strong mitochondrial activity, as well as modulating anti-inflammatory responses [172]. It is noteworthy that 
in Pink1 $^{-/-}$flies the neurodegenerative process was shown to depend on the oxidative-stress-triggered inactivation of the labile [4Fe-4S] clusters in the aconitase enzymes [32].

Since iron accumulation is neurotoxic over time [173] and correlates with iron consumption from red meat and bread [174], our observations may also be relevant for dietary approaches that might mitigate disease progression in PD. In the context of dietary prevention of PD, it is important to note that ascorbate (vitamin C) is a modulator of cellular iron import and efflux as well as ferritin biosynthesis and degradation [175-180]. Thus, our data may provide a preliminary mechanistic concept to explain the accumulation of iron deposits in the brain of PD patients with PINK1 loss-of-function mutations. In-depth analyses with overexpression and repression of key molecules in brain glia versus neural cells will be necessary to assess this putative scenario. In this context, a recently published study of Pink $1^{-1}$ flies demonstrated increased iron bioavailability in mitochondria via mitoferrin overexpression or ferritin knockdown to be beneficial for motor performance and mitochondrial morphology [181], an observation that contradicts the assumption of iron chelator benefits in PD. The enhanced sensitivity of PINK1-deficient cells to iron overload/toxicity and ferroptosis with reduced lifespan of such organisms might constitute an adverse effect triggered by mechanisms to maximize iron recruitment for inefficient mitochondrial usage.

This study elucidated not only the molecular effects of the PINK1/PARKIN pathway as intrinsic determinants of mitophagy, PD, and longevity. In addition, an important focus of our study was on iron as an extrinsic modulator of these mitochondrial functions, and on the homeostatic expression adaptations that are physiologically induced by iron. Important responses to iron depletion included the slight upregulation of $A b c b 10$ mRNA contrasted by a converse downregulation of Slc25a28 (mitoferrin-2), although both transporters are thought to mediate iron import. The substrates of ABCB10 transport activity are currently undefined, but its absence was reported to reduce mitoferrin-1 protein levels, iron import into mitochondria, heme biosynthesis, and hemoglobinization, while a role in the export of ALA (5'-aminolevulinic acid) was excluded [182]. It is difficult to identify the individual substrates for each mitochondrial transporter protein, given that $\mathrm{ABCB} 10$, the putative iron-importer mitoferrin, the heme-synthesis factor ferrochelatase, and the ISC-exporter ABCB7 coexist in a protein complex $[72,79,83,183]$ where the deletion of one member may destabilize also its interactors. Similar to ABCB10, a decrease of iron import and heme biosynthesis was also shown upon deletion of mitoferrin [184,185], leading to universal acceptance of mitoferrin as the main mitochondrial iron importer [84]. Upon comparison of the transcriptional regulation of both factors, it is intriguing to note that iron shortage leads to parallel induction of Tfrc for iron recruitment across plasma membranes, and induction of $A b c b 10$ that could recruit iron across mitochondrial membranes, but a converse downregulation of Slc25a28 which encodes mitoferrin-2. This paradoxical Slc25a28 downregulation during iron shortage, however, might be expected for a transporter that mediates iron export but is unusual for an import factor. The FAC-triggered upregulation of Slc25a37, which encodes mitoferrin-1, at least in Pink1 $^{-/}$cells (Supplementary Figure S5), is expected for a mitochondrial iron import factor, and compatible with the idea of maximized iron recruitment when ISC-biogenesis is impaired. Interestingly, the reduction of Slc25a28 in C. elegans was reported to result in a prolonged lifespan [74]. In only one study so far, ABCB8 was implicated in mitochondrial export functions for iron and factors required for cytosolic ISC-protein maturation [80]. As expected for mitochondrial iron export factors, $A b c b 8$ expression was downregulated after iron depletion, to a similar level as Slc25a28. Regarding the two mitoferrin isoforms, it was also noteworthy that $S l c 25 a 28$ responded to iron depletion, whereas Slc25a37 seemed regulated only after iron excess (Figure S5). Thus, it is also possible that mitoferrin 1 may be better suited to importing iron into mitochondria under conditions of high iron supply (as is the case in erythroid cells in which mitoferrin 1 is the major mitochondrial iron importer). We propose that further studies of transcriptional regulation in response to putative substrate loading would help to elucidate the specific roles of each membrane transporter. 


\section{Materials and Methods}

\subsection{Mouse Embryonic Fibroblast Generation and Culture}

The mice used were bred at the Central Animal Facility (ZFE) of the Goethe University Medical Faculty in Frankfurt, under FELASA-certified conditions, in accordance with the ETS123 (European Convention for the Protection of Vertebrate Animals), the Council Directive of 24 November 1986 (86/609/EWG) with Annex II and the German Animal Welfare Act. Approval of the local institutional review board (Regierungspraesidium Darmstadt, project V54-19c20/15-FK/1083) was given on 27 March 2017. MEF were prepared from individual embryos at 14.5 days post-coitus of WT and Pink1 ${ }^{-/-}$mice, which were generated and bred as previously reported [20]. In brief, embryos were dissected from the uterus, extremities, and inner organs were removed and the tissue was treated with $0.05 \%$ trypsin (Gibco, Thermo Scientific, Schwerte, Germany) for 10-15 min. Cells were cultivated in Dulbecco's Modified Eagle Medium $4.5 \mathrm{~g} / \mathrm{L}$ glucose (Invitrogen, Karlsruhe, Germany) plus 15\% bovine growth serum (BGS, Thermo Scientific), $1 \%$ glutamine, $1 \%$ penicillin/streptomycin (all Invitrogen,) at $37^{\circ} \mathrm{C}$ and $5 \% \mathrm{CO}_{2}$ in a humidified incubator, then Pink1 ${ }^{-/-}$cells and their respective littermate WT controls were frozen in liquid nitrogen.

\subsection{Human Fibroblasts}

Human fibroblasts of 7 healthy controls and 3 PARK6 patients [17] (passage 8-12) were cultured in Dulbecco's Modified Eagle Medium $4.5 \mathrm{~g} / \mathrm{L}$ glucose (Invitrogen) plus 15\% bovine growth serum (BGS, Thermo Scientific), $1 \%$ glutamine, $1 \%$ penicillin/streptomycin (all Invitrogen) at $37^{\circ} \mathrm{C}$ and $5 \%$ $\mathrm{CO}_{2}$ in a humidified incubator.

\subsection{Iron Overload/Depletion Experiments}

MEF or human fibroblast cells were plated in 6-well plates (500,000 cells/well for RT-qPCR and immunoblotting, $1 \times 10^{6}$ for global proteome) and incubated for $24 \mathrm{~h}$. Cells were either left in fresh normal growth medium or treated with $200 \mu \mathrm{M}$ ferric ammonium citrate (FAC) (Sigma Aldrich, St. Louis, MO, USA), 100 M 2,2-bipyridyl (22BP) (Roth, Karlsruhe, Germany), or $100 \mu$ M Deferoxamine mesylate (DFO) (Sigma Aldrich) in their normal culture medium. Incubation was done for $16 \mathrm{~h}$ or $48 \mathrm{~h}$ and cell pellets were collected for subsequent RNA and protein isolation, respectively.

\subsection{Reverse Transcriptase Real-Time Quantitative PCR}

For isolation of total RNA, TRI reagent (Sigma Aldrich) was used, and VILO IV (Thermo Scientific) for reverse transcription, both following manufacturers' instructions. RT-qPCR was performed applying TaqMan Gene Expression Assays (Applied Biosystems, Thermo Scientific) in cDNA from $20 \mathrm{ng}$ total RNA in $20 \mu \mathrm{L}$ reactions with $2 \times$ master mix (Roche, Basel, Switzerland) in a StepOnePlus Real-Time PCR System (Applied Biosystems, Thermo Scientific). An RT-qPCR assay of Pink1 normalized to Tbp was used to confirm the genotype in MEFs. For quantification of the individual mRNA levels, the following TaqMan assays (Thermo Scientific) were employed for mouse: Abcb6- Mm00470049_m1, Abcb7- Mm01235258_m1, Abcb8- Mm00472410_m1, Abcb10Mm00497931_m1, Abce1- Mm00649858_m1, Aco1- Mm00801417_m1, Aco2- Mm00475673_g1, Alas1Mm01235914_m1, Bach1-Mm01344527_m1, Bdh2- Mm00459075_m1, Bnip3- Mm00833810_g1, Bola1Mm01255885_m1, Brip1- Mm01297848_m1, Cdc42bpa- Mm01322796_m1, Cisd1- Mm00728581_s1, Cisd2-Mm00835272_m1, Cp-Mm00432654_m1, Ctsb-Mm01310605_m1, Ctsd-Mm00515586_m1,CtsfMm00490782_m1, Cygb-Mm00446071_m1, Cyp46a1 -Mm00487306_m1, Dna2- Mm01169107_m1, DpydMm00468109_m1, Egln1- Mm00459770_m1, Elp3- Mm00804536_m1, Ercc2- Mm00514776_m1, Fdx1Mm00433246_m1, Fech- Mm00500394_m1, Flvcr1- Mm01320423_m1, Foxo3- Mm01185722_m1, Fth1Mm00850707_m1, Ftl1- Mm03030144_g1, Fxn- Mm00784016_s1, Gabarapl1- Mm00457880_m1, Glrx5Mm00511712_m1, Hebp1-Mm00469161_m1, Hif1a- Mm00468869_m1, Hk1- Mm00439344_m1, Homer1Mm00516275_m1, Hmox1-Mm00516005_m1, Ireb2- Mm01179595_m1, Jmjd6- Mm00466679_m1, Jund- 
Mm04208316_m1, Keap1-Mm00497268_m1, Mef2d-Mm00504931_m1, Mitf-Mm00434954_m1,Mmp14Mm00485054_m1, Myl6-Mm02342525_g1, Ncoa4- Mm00451095_m1, Nfe2l2- Mm00477784_mL, Nfu1Mm00777068_m1, Nos2- Mm_00440502_m1, Nqo1- Mm01253561_m1, Nthl1- Mm00476559_m1, P4ha2Mm01288628_m1, Prkn- Mm00450186_m1, Pcbp1- Mm00478712_s1, Pcbp2- Mm01296174_g1, Pcbp3Mm01149750_m1, Pgrmc1- Mm00443985_m1, Pink1- Mm00550827_m1, Pold1- Mm00448253_m1, PpatMm00549096_m1, Prdx1- Mm012619961_s1, Prim2- Mm00477104_m1, Rbfox2- Mm01197021_m1, Rsad1Mm01296523_m1, Rsad2- Mm00491265_m1, Rrm2- Mm00485881_m1, Rtel1- Mm01220420_m1, Slc11a2Mm00435363_m1, Slc25a28-Mm00455077_m1, Slc25a37- Mm00471133_m1, Slc40a1- Mm01254822_m1, Sqstm1-Mm00448091_m1, Steap2-Mm01320129_m1, Steap3- Mm01287243_m1, Tbp-Mm00446973_m1, Tfeb- Mm00448968_m1, Tfrc-Mm00441941_m1, Trf-Mm00446715_m1, Tyw5- Mm01254171_m1. Human TaqMan assays used were: ABCE1- Hs01009190_m1, FOXO3- Hs00921424_m1, FTL1- Hs00830226_gH, FTH1- Hs01694011_s1, HPRT1- Hs99999909_m1, KEAP1- Hs00202227_m1, RRM2- Hs00357247_g1, TFEB-Hs00292981_m1, TFRC-Hs00951083_m1. Results were analyzed with the $2^{-\Delta \Delta C T}$ method [186].

\subsection{Quantitative Immunoblotting}

Sample preparation for quantitative immunoblotting was done as described before [187]. Samples of $20 \mu \mathrm{g}$ of protein in $2 \times$ Laemmli buffer were heated at $90{ }^{\circ} \mathrm{C}$ for $3 \mathrm{~min}$ and then separated in $10 \%$ tris-glycine polyacrylamide gels, using Precision Plus Protein ${ }^{\mathrm{TM}}$ All Blue Standards (Bio-Rad, Hercules, CA, USA) as a size marker. Transfer to nitrocellulose membranes (Protran, GE Healthcare, Thermo Fisher) was done at $50 \mathrm{~V}$ over $90 \mathrm{~min}$, with blocking in 5\% BSA solution in $1 \times$ TBS-T for $1 \mathrm{~h}$ at room temperature (RT). Primary antibody incubation against FTH1 (1:1000, Invitrogen, \#PA586928), HMOX1 (1:1000, Abcam, Cambridge, UK, ab79854), RRM2 (1:500, Santa Cruz Biotechnolgoy, Santa Cruz, CA, USA, sc-376973), and NCOA4 (1:1000, Santa Cruz sc-373739). Fluorescence-labeled $\alpha$-rabbit or $\alpha$-mouse antibodies (1:15,000, Licor Biosciences, Lincoln, NE, USA) were used as secondary antibodies. Normalization occurred with incubation against beta-Actin (1:2000, Sigma Aldrich, A5441). Fluorescence detection occurred on the Licor Odyssey Classic Instrument and bands were densitometrically analyzed with Image Studio Lite, Version 5.2 (Li-Cor Biosciences).

\subsection{Proteomics Sample Preparation with Label-Free Quantification (LFQ)}

Proteomics sample preparation was done according to a published protocol with minor modifications [188]. About 1.5 million cells of Pink1 ${ }^{-/-}$MEF and WT cells, either FAC, 22BP, or untreated were lysed in triplicates under denaturing conditions in a buffer containing $3 \mathrm{M}$ guanidinium chloride (GdmCl), $10 \mathrm{mM}$ tris(2-carboxyethyl)phosphine, $40 \mathrm{mM}$ chloroacetamide and $100 \mathrm{mM}$ Tris- $\mathrm{HCl}$ $\mathrm{pH}$ 8.5. Lysates were denatured at $95^{\circ} \mathrm{C}$ for $10 \mathrm{~min}$ shaking at $1000 \mathrm{rpm}$ in a thermal shaker and sonicated for $10 \mathrm{~min}$. Lysates were diluted with a dilution buffer containing $10 \%$ acetonitrile and $25 \mathrm{mM}$ Tris- $\mathrm{HCl}, \mathrm{pH}$ 8.0, to reach a $1 \mathrm{M} \mathrm{GdmCl}$ concentration. Then, proteins were digested with LysC (Roche; enzyme to protein ratio 1:50, MS-grade) shaking at $700 \mathrm{rpm}$ at $37^{\circ} \mathrm{C}$ for $2 \mathrm{~h}$. The digestion mixture was diluted again with the same dilution buffer to reach $0.5 \mathrm{M} \mathrm{GdmCl}$, followed by a tryptic digestion (Roche, enzyme to protein ratio 1:50, MS-grade) and incubation at $37^{\circ} \mathrm{C}$ overnight in a thermal shaker at $700 \mathrm{rpm}$. Peptide desalting was performed according to the manufacturer's instructions (Pierce C18 Tips, Thermo Scientific, Waltham, MA, USA). Desalted peptides were reconstituted in $1 \%$ formic acid in water and half of each sample was further separated into four fractions by strong cation exchange chromatography (SCX, 3M Purification, Meriden, CT, USA). Eluates were first dried in a SpeedVac, then dissolved in $5 \%$ acetonitrile and $2 \%$ formic acid in water, briefly vortexed, and sonicated in a water bath for $30 \mathrm{~s}$ prior injection to nano-LC-MS.

\subsection{LC-MS/MS Instrument Settings for Shotgun Proteome Profiling and Data Analysis}

LC-MS/MS was carried out by nanoflow reverse phase liquid chromatography (Dionex Ultimate 3000, Thermo Scientific) coupled online to a Q-Exactive HF Orbitrap mass spectrometer (Thermo Scientific), as reported previously [189]. Briefly, the LC separation was performed using a PicoFrit 
analytical column (75 $\mu \mathrm{m}$ ID $\times 50$ cm long, $15 \mu \mathrm{m}$ Tip ID; New Objectives, Woburn, MA, USA) in-house packed with 3- $\mu \mathrm{m}$ C18 resin (Reprosil-AQ Pur, Dr. Maisch, Ammerbuch, Germany). Peptides were eluted using a gradient from $3.8 \%$ to $38 \%$ solvent $\mathrm{B}$ in solvent A over $120 \mathrm{~min}$ at $266 \mathrm{~nL}$ per minute flow rate. Solvent A was $0.1 \%$ formic acid and solvent $\mathrm{B}$ was $79.9 \%$ acetonitrile, $20 \% \mathrm{H}_{2} \mathrm{O}, 0.1 \%$ formic acid. For the IP samples, a one hour gradient was used. Nanoelectrospray was generated by applying $3.5 \mathrm{kV}$. A cycle of one full Fourier transformation scan mass spectrum $(300-1750 \mathrm{~m} / \mathrm{z}$, resolution of 60,000 at $\mathrm{m} / \mathrm{z} 200$, automatic gain control (AGC) target $1 \times 10^{6}$ ) was followed by 12 data-dependent MS/MS scans (resolution of 30,000, AGC target $5 \times 10^{5}$ ) with a normalized collision energy of $25 \mathrm{eV}$. In order to avoid repeated sequencing of the same peptides, a dynamic exclusion window of $30 \mathrm{~s}$ was used. In addition, only peptide charge states between two to eight were sequenced.

Raw MS data were processed with MaxQuant software (v1.6.10.43, from Max Planck Institute of Biochemistry, Martinsried, Germany) and searched against the mouse proteome database UniProtKB with 55,153 entries, released in August 2019. Parameters of MaxQuant database searching were a false discovery rate (FDR) of 0.01 for proteins and peptides, a minimum peptide length of seven amino acids, a first search mass tolerance for peptides of $20 \mathrm{ppm}$ and a main search tolerance of $4.5 \mathrm{ppm}$, and using the function "match between runs". A maximum of two missed cleavages was allowed for the tryptic digest. Cysteine carbamidomethylation was set as fixed modification, while N-terminal acetylation and methionine oxidation were set as variable modifications. The correlation analysis of biological replicates and the calculation of significantly different proteins were done with Perseus (v1.6.10.43, from Max Planck Institute of Biochemistry). LFQ intensities, originating from at least two different peptides per protein group were transformed by $\log _{2}$. Only groups with valid values in at least one group were used, missing values were replaced by values from the normal distribution. Statistical analysis was done by a two-sample t-test with Benjamini-Hochberg (BH, FDR of 0.05) correction for multiple testing. Significantly regulated proteins between the conditions were indicated by a plus sign in Supplementary Table S1.

\subsection{Statistical Evaluation}

RT-qPCR and quantitative immunoblot result were analyzed using Graphpad Prism Version 8 (GraphPad, San Diego, CA, USA) and significant differences were calculated with two-way ANOVA with subsequent multiple comparison tests.

\subsection{Visualization}

Graphs were created using Graphpad Prism Version 8; Tables were assembled using Microsoft Excel, and Venn diagrams were created with the online tool for Venn diagrams of Bioinformatics \& Evolutionary Genomics (http://bioinformatics.psb.ugent.be/webtools/Venn/, accessed on 18.09.2020)

\section{Conclusions}

We conducted a pioneering survey of global proteomic adaptations and transcriptional responses to extracellular iron changes, using murine fibroblasts from WT and Pink1 $1^{-/}$animals, to then validate prominent findings in fibroblasts from PARK6 patients. The proteome profile identified 4 novel factors to be conversely regulated upon iron deficiency versus iron excess, namely CPT1A, MMP14, XDH, and PYGL. The transcriptional regulation profile argued against previous notions that SLC25A28 has mitochondrial import function, providing evidence that SLC25A28 expression is downregulated by iron shortage, while SLC25A37 responds to iron excess.

Iron deprivation had massive effects on proteome and transcript levels and they are relevant when trying long-term neuroprotective therapy of PD patients by iron chelators. On the one hand, iron shortage upregulated the alpha-synuclein antagonist SNCB at the protein level, and Pink1 and Prkn at the mRNA level, possibly ensuring neuroprotection and chronic maximized mitophagy, which might have beneficial effects for the aggregation and mitochondrial dysfunction in PD tissue. On the other hand, iron shortage decreased the abundance of iron-binding nucleotide synthesis factors such 
as RRM2, of ISC biogenesis factors, and ISC-associated guardians of RNA/DNA stability, limiting also the transcriptional expression e.g., for Ppat, Nthl1, Dna2, Pold1, Tyw5, and Prim2, so the known vulnerability of the mitochondrial genome in PD might be enhanced in deleterious fashion by iron chelators. Particularly the impairment of DNA repair is known as a risk factor of neurodegenerative diseases and a modulator of lifespan [13-15]. Largely, the factors identified act via iron homeostasis and mitophagy to alter the health period [11,12].

Even at untreated conditions, the Pink1-ablation mediated a deficit of the LIP chaperone PCBP3 protein, the ISC-biogenesis factor GLRX5 protein, and the ISC-associated Abce1 transcript as a growth-limiting effect. These data provide the first evidence in mammals for the existence of a PINK1-dependent mechanism described previously in D. melanogaster $[122,124,148]$, where nucleus-encoded mRNAs and the corresponding precursor proteins for mitochondria undergo co-translational quality control by $\mathrm{ABCE1}$, in parallel to the mitophagic elimination of dysfunctional mitochondrial fragments.

As a prominent finding after iron overload, the transcriptional ferritin induction was potentiated in Pink1-ablated cells, while the ferritin protein levels were diminished, a contrast that might be due to post-transcriptional translation repression caused by reduced ISC-availability/stability.

Taken together, this preliminary documentation of on-demand regulations in fibroblasts should be complemented by studies of neural cells, mitochondrial fractions, long-term effects, and overexpression/depletion of crucial factors, to confirm the mechanisms and the relevance for PD. The data point to inefficient iron usage in the mitochondria of PINK1-mutant cells, followed by maximized iron import and mobilization to ensure sufficient DNA/RNA quality, via ISC-associated enzymes. This observation clarifies in what pathways an iron chelator therapy of PD may have long-term adverse effects and explains why acutely improved motor performance can be observed in PINK1-mutant flies after iron supplementation.

Supplementary Materials: The following are available online at http://www.mdpi.com/2073-4409/9/10/2229/s1. Table S1, Perseus software output of global proteome analyses; Table S2, Statisctical details of transcriptional changes; Figure S1, Dysregulations in global proteome of WT MEF after FAC-treatment; Figure S2, Volcano plots of global proteome profiles; Figure S3, STRING diagram of consistently dysregulated factors; Figure S4, Dysregulations in global proteome of WT MEF after 22BP-treatment; Figure S5, Transcriptional dysregulations upon RT-qPCR analyses; Figure S6, Transcriptional dysregulations of antioxidant and hypoxic control.

Author Contributions: Conceptualization, G.A.; methodology, G.A., J.K., N.E.S., D.M., A.A., S.K., R.H., N.N.K., G.K., S.G.; writing - original draft preparation, G.A., J.K.; writing-review and editing, all authors; funding acquisition, G.A. All authors have read and agreed to the published version of the manuscript.

Funding: This research was funded by the German Federal Ministry of Education through the National Genome Research Network (NGFNplus, Bundesministerium für Bildung und Forschung, 01GS08138), the GerontoMitoSys network (Bundesministerium für Bildung und Forschung, PTJ 0315584A) and by the European Union (ERAnet-RePARK, DLR 01EW1012).

Acknowledgments: The authors thank Prof. Roland Lill and Dr. Oliver Stehling for helpful discussions and providing antibodies and Beata Lukaszewska-McGreal for proteome sample preparation. We are grateful to Antonius Auburger and Georg Auburger junior for bioinformatics assistance.

Conflicts of Interest: The authors declare no conflict of interest.

\section{Abbreviations}

$\begin{array}{ll}\mu \mathrm{M} & \text { MicroMolar } \\ \text { 22BP } & \text { 2,2'-Bipyridine } \\ \text { Abcb1 } & \text { ATP binding cassette subfamily B member 1 } \\ \text { Abcb7 } & \text { ATP binding cassette subfamily B member 7 } \\ \text { Abcb8 } & \text { ATP binding cassette subfamily B member } 8 \\ \text { Abcb10 } & \text { ATP binding cassette subfamily B member 10 } \\ \text { Abce1 } & \text { ATP binding cassette subfamily E member 1, RNase L inhibitor 1 } \\ \text { Aco1 } & \text { Aconitase 1, cytoplasmic, aka Irp1, aka Ferritin repressor protein }\end{array}$




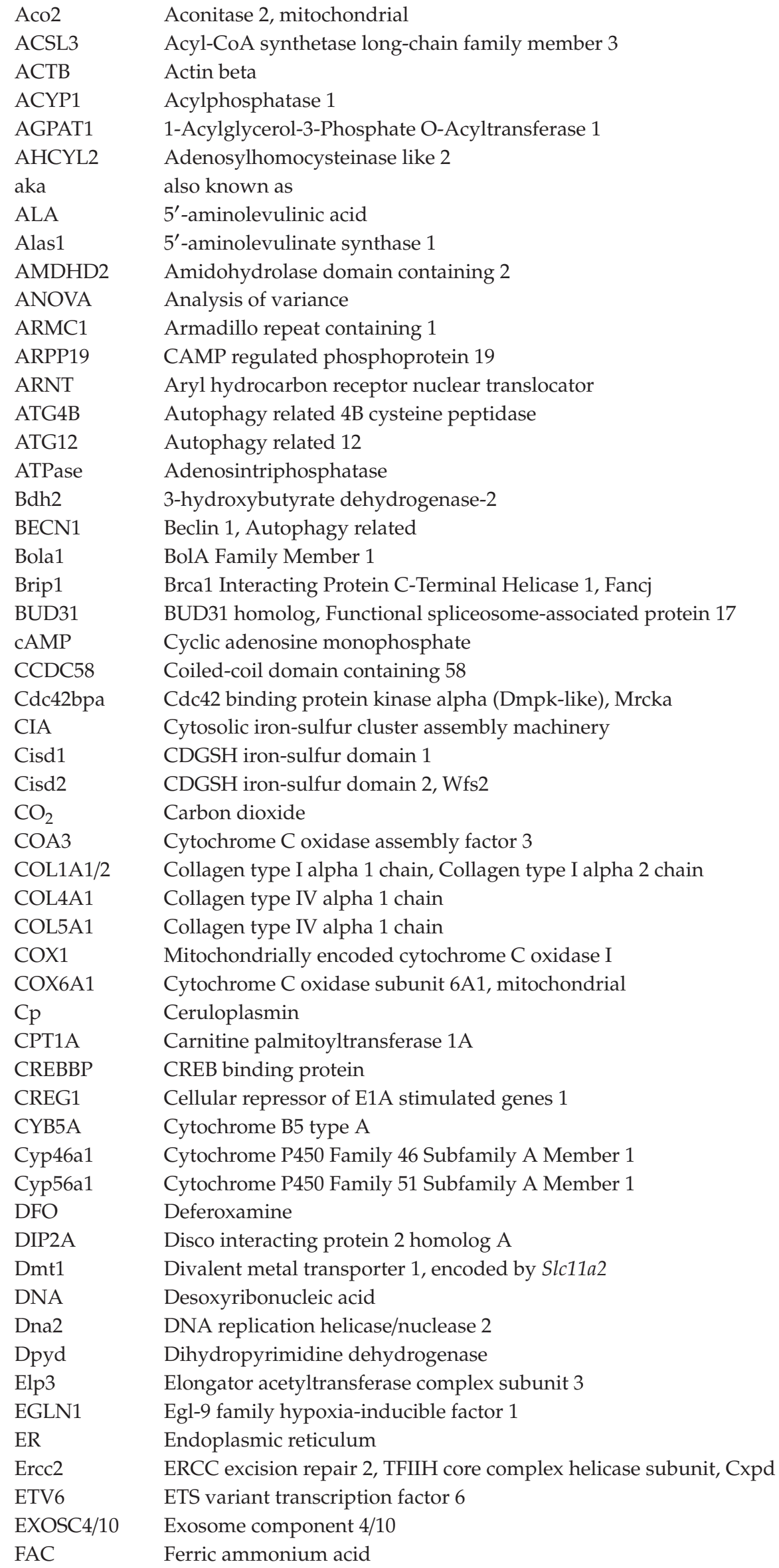




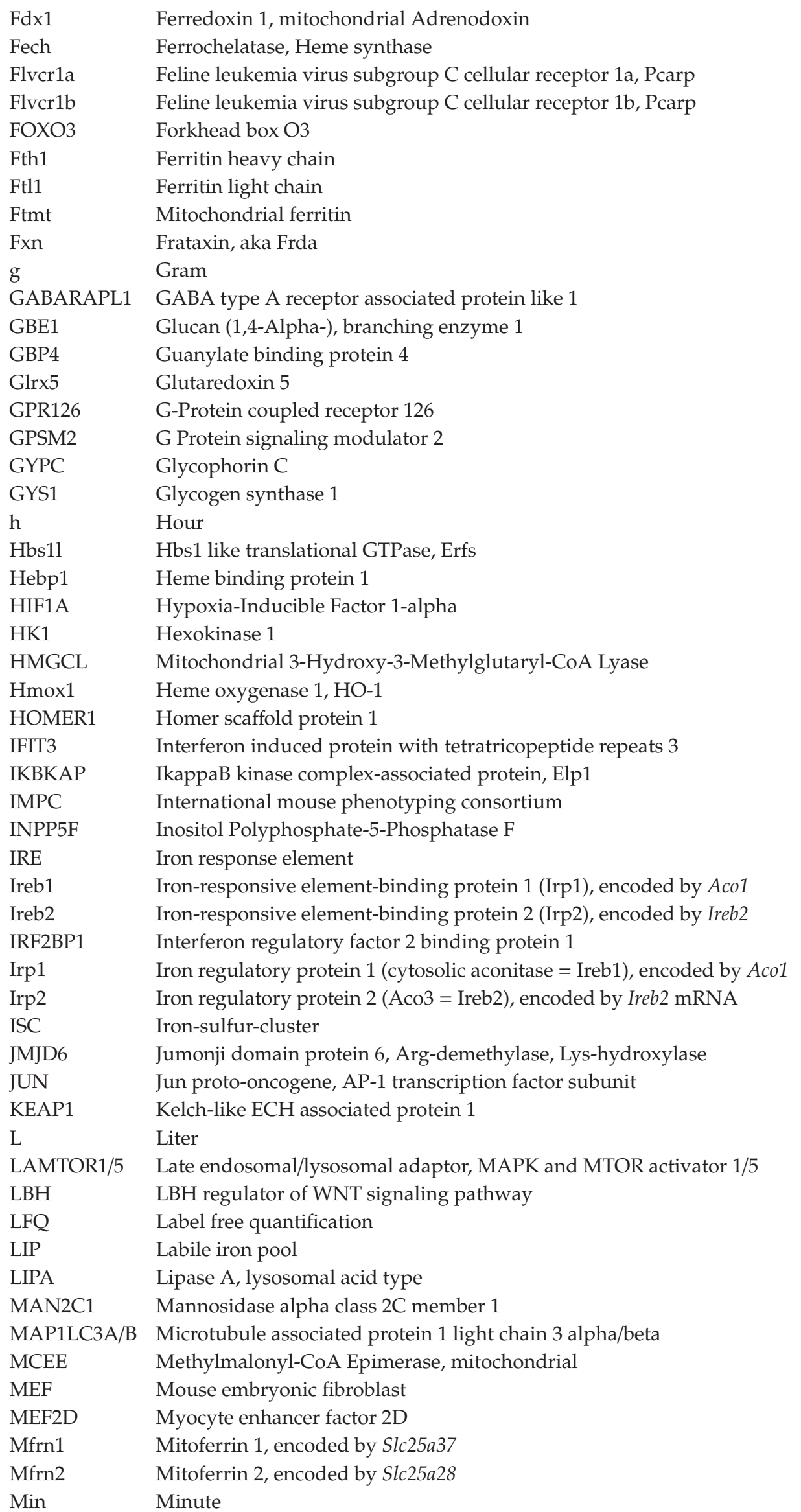




\begin{tabular}{|c|c|}
\hline MMP14 & Matrix metallopeptidase 14 \\
\hline MRPS36 & Mitochondrial ribosomal protein S36 \\
\hline MSRA & Methionine sulfoxide reductase A \\
\hline mRNA & Messenger ribonucleic acid \\
\hline mTOR & Mechanistic target of rapamycin kinase \\
\hline MPTP & 1-methyl-4-phenyl-1,2,3,6-tetrahydropyridine \\
\hline MYD88 & Myeloid differentiation primary response protein MyD88 \\
\hline MYL6 & Myosin light chain 6 \\
\hline Ncoa4 & Nuclear receptor coactivator 4 \\
\hline NDUFA10 & NADH:Ubiquinone oxidoreductase subunit A10 \\
\hline NDUFS3/8 & NADH:Ubiquinone oxidoreductase core subunit S3/subunit S8 \\
\hline NFkB & Nuclear Factor 'kappa-light-chain-enhancer' of activated B-cells \\
\hline Nfu1 & Nfu1 iron-sulfur cluster scaffold \\
\hline NMRAL1 & NmrA like redox sensor 1 \\
\hline NOS2 & Nitric oxide synthase 2 \\
\hline Not4 & Ccr4-Not transcription complex subunit 4 \\
\hline NQO1 & $\mathrm{NAD}(\mathrm{P}) \mathrm{H}$ quinone dehydrogenase 1 \\
\hline NRF2 & Nuclear factor, erythroid 2 like 2, Nfe2l2 \\
\hline NSMCE2 & Non-structural maintenance of chromosomes element 2 homolog \\
\hline Nthl1 & Nth like DNA glycosylase 1 \\
\hline NUCKS1 & Nuclear casein kinase and cyclin-dependent kinase substrate 1 \\
\hline NUP160 & Nucleoporin 160 \\
\hline OAS1G & $2^{\prime}-5^{\prime}$-Oligoadenylate synthetase 1 \\
\hline P4HA2 & Prolyl 4-hydroxylase subunit alpha 2 \\
\hline PANK4 & Pantothenate kinase 4 \\
\hline Parkin & Parkinson disease protein 2 \\
\hline PAWR & Pro-apoptotic WT1 regulator \\
\hline Pcbp1 & Poly(RC) binding protein 1 , hnRNP-E1 \\
\hline Pcbp2 & Poly $(\mathrm{RC})$ binding protein 2 , hnRNP-E2 \\
\hline PD & Parkinson's disease \\
\hline PDDC1 & Glutamine amidotransferase like Class 1 domain containing 1, Gatd1 \\
\hline Pgrmc1 & Progesterone receptor membrane component 1, Dap1 \\
\hline Pink1 & PTEN induced kinase 1 \\
\hline PLOD1 & Procollagen-lysine,2-oxoglutarate 5-dioxygenase 1 \\
\hline PMPCA & Peptidase, mitochondrial processing subunit alpha \\
\hline Pold1 & DNA polymerase delta 1 catalytic subunit, Cdc2 homolog \\
\hline POMP & Proteasome maturation protein \\
\hline Ppat & Phosphoribosyl pyrophosphate amidotransferase, Gpat, ATase \\
\hline Prim2 & DNA primase subunit 2 \\
\hline PTGES & Prostaglandin E synthase \\
\hline PMID & PubMed database of medical literature, reference IDentifier number \\
\hline PYGL & Glycogen Phosphorylase L \\
\hline RBFOX2 & RNA binding fox-1 homolog 2 \\
\hline RNA & Ribonucleic acid \\
\hline ROS & Reactive oxygen species \\
\hline RPF2 & Ribosome production factor 2 homolog \\
\hline RRM1/2 & Ribonucleotide reductase regulatory subunit M1/M2 \\
\hline rRNA & Ribosomal RNA \\
\hline Rsad1 & Radical S-adenosyl methionine domain containing 1 \\
\hline Rsad2 & Radical S-adenosyl methionine domain containing 2 \\
\hline RT & Room temperature \\
\hline RT-qPCR & reverse-transcriptase real-time quantitative polymerase chain reaction \\
\hline Rtel1 & Regulator of telomere elongation helicase 1 \\
\hline SCCPDH & Saccharopine dehydrogenase \\
\hline
\end{tabular}




\begin{tabular}{|c|c|}
\hline SEM & Standard error of the mean \\
\hline SELENBP1 & Selenium binding protein 1 \\
\hline SERPINE2 & Serpin family E member 2 \\
\hline SH2B1 & $\mathrm{SH} 2$ domain-containing protein $1 \mathrm{~B}$ \\
\hline Slc11a2 & Solute carrier family 11 member 2, Divalent metal transporter 1, Dmt1 \\
\hline Slc25a37 & Solute carrier family 25 member 37, Mitoferrin 1 \\
\hline Slc25a28 & Solute carrier family 25 member 28 , Mitoferrin 2 \\
\hline Slc40a1 & Solute carrier family 40 member 1, Ferroportin 1, Ireg1 \\
\hline SNCB & Synuclein beta \\
\hline SMX30 & Sorting nexin family member 30, ATG24A \\
\hline SPP1 & Secreted phosphoprotein 1 \\
\hline SRSF10 & Serine and arginine-rich splicing factor 10 \\
\hline SSBP1 & Single-stranded DNA binding protein 1 \\
\hline Steap2 & Six transmembrane epithelial antigen of the prostate 2, Stmp \\
\hline Steap3 & Six transmembrane epithelial antigen of the prostate 3 , Stmp3 \\
\hline Steap4 & Six transmembrane epithelial antigen of the prostate 4 , Stamp2 \\
\hline STRING & Search Tool for the Retrieval of Interacting Genes/Proteins \\
\hline SYN1 & Synapsin I \\
\hline Tbp & Tata-binding protein \\
\hline TBST & Tris-buffered saline/Tween 20 \\
\hline TCA & Tricarboxylic acid cycle \\
\hline TCIRG1 & T Cell immune regulator 1 , ATPase $\mathrm{H}+$ transporting V0 subunit A3 \\
\hline TIMM10 & Translocase of inner mitochondrial membrane 10 \\
\hline TMEM115 & Transmembrane protein 115 \\
\hline TMEM173 & Stimulator of interferon genes protein, Sting \\
\hline TMEM63A & Transmembrane protein $63 \mathrm{~A}$ \\
\hline TRAF2 & TNF receptor-associated factor 2 \\
\hline TREX1 & Three prime repair exonuclease 1 \\
\hline $\operatorname{Trf}$ & Transferrin \\
\hline Tfre & Transferrin receptor 1 \\
\hline Tyw5 & tRNA wybutosine synthesizing protein 5 \\
\hline UPP1 & Uridine phosphorylase 1 \\
\hline UTR & Untranslated region \\
\hline $\mathrm{V}$ & Volt \\
\hline VHL & Von Hippel-Lindau tumor suppressor \\
\hline WT & Wildtype \\
\hline XAF1 & XIAP associated factor 1 \\
\hline $\mathrm{XDH}$ & Xanthine dehydrogenase \\
\hline ZBP1 & Z-DNA binding protein 1 \\
\hline
\end{tabular}

\section{References}

1. Weiland, A.; Wang, Y.; Wu, W.; Lan, X.; Han, X.; Li, Q.; Wang, J. Ferroptosis and Its Role in Diverse Brain Diseases. Mol. Neurobiol. 2019, 56, 4880-4893. [CrossRef] [PubMed]

2. Stehling, O.; Lill, R. The Role of Mitochondria in Cellular Iron-Sulfur Protein Biogenesis: Mechanisms, Connected Processes, and Diseases. Cold Spring Harb. Perspect. Biol. 2013, 5, a011312. [CrossRef] [PubMed]

3. Stehling, O.; Wilbrecht, C.; Lill, R. Mitochondrial iron-sulfur protein biogenesis and human disease. Biochimie 2014, 100, 61-77. [CrossRef] [PubMed]

4. Kafina, M.D.; Paw, B.H. Intracellular iron and heme trafficking and metabolism in developing erythroblasts. Metallomics 2017, 9, 1193-1203. [CrossRef]

5. Barupala, D.P.; Dzul, S.P.; Riggs-Gelasco, P.J.; Stemmler, T.L. Synthesis, delivery and regulation of eukaryotic heme and Fe-S cluster cofactors. Arch. Biochem. Biophys. 2016, 592, 60-75. [CrossRef]

6. Kimura, S.; Suzuki, T. Iron-sulfur proteins responsible for RNA modifications. Biochim. Biophys. Acta (BBA)-Bioenerg. 2015, 1853, 1272-1283. [CrossRef] 
7. Lill, R. Function and biogenesis of iron-sulphur proteins. Nature 2009, 460, 831-838. [CrossRef]

8. Puig, S.; Ramos-Alonso, L.; Romero, A.M.; Martínez-Pastor, M.T. The elemental role of iron in DNA synthesis and repair. Metallomics 2017, 9, 1483-1500. [CrossRef]

9. Paul, V.D.; Lill, R. Biogenesis of cytosolic and nuclear iron-sulfur proteins and their role in genome stability. Biochim. Biophys. Acta (BBA) 2015, 1853, 1528-1539. [CrossRef]

10. Furuyama, K.; Kaneko, K.; Vargas, P.D. Heme as a Magnificent Molecule with Multiple Missions: Heme Determines Its Own Fate and Governs Cellular Homeostasis. Tohoku J. Exp. Med. 2007, 213, 1-16. [CrossRef]

11. Schiavi, A.; Maglioni, S.; Palikaras, K.; Shaik, A.; Strappazzon, F.; Brinkmann, V.; Torgovnick, A.; Castelein, N.; De Henau, S.; Braeckman, B.P.; et al. Iron-Starvation-Induced Mitophagy Mediates Lifespan Extension upon Mitochondrial Stress in C. elegans. Curr. Biol. 2015, 25, 1810-1822. [CrossRef] [PubMed]

12. Ryu, D.; Mouchiroud, L.; Andreux, P.A.; Katsyuba, E.; Moullan, N.; A Nicolet-Dit-Félix, A.; Williams, E.G.; Jha, P.; Sasso, G.L.; Huzard, D.; et al. Urolithin A induces mitophagy and prolongs lifespan in C. elegans and increases muscle function in rodents. Nat. Med. 2016, 22, 879-888. [CrossRef] [PubMed]

13. Corti, O.; Lesage, S.; Brice, A. What Genetics Tells us About the Causes and Mechanisms of Parkinson's Disease. Physiol. Rev. 2011, 91, 1161-1218. [CrossRef] [PubMed]

14. Valente, E.M.; Abou-Sleiman, P.M.; Caputo, V.; Muqit, M.M.K.; Harvey, R.J.; Gispert, S.; Ali, Z.; Del Turco, D.; Bentivoglio, A.R.; Healy, D.G.; et al. Hereditary Early-Onset Parkinson's Disease Caused by Mutations in PINK1. Science 2004, 304, 1158-1160. [CrossRef] [PubMed]

15. Exner, N.; Treske, B.; Paquet, D.; Holmstrom, K.; Schiesling, C.; Gispert, S.; Carballo-Carbajal, I.; Berg, D.; Hoepken, H.-H.; Gasser, T.; et al. Loss-of-Function of Human PINK1 Results in Mitochondrial Pathology and Can Be Rescued by Parkin. J. Neurosci. 2007, 27, 12413-12418. [CrossRef]

16. Matsuda, N.; Tanaka, K. Uncovering the roles of PINK1 and parkin in mitophagy. Autophagy 2010, 6, 952-954. [CrossRef] [PubMed]

17. Hoepken, H.-H.; Gispert, S.; Morales, B.; Wingerter, O.; Del Turco, D.; Mülsch, A.; Nussbaum, R.L.; Müller, K.; Dröse, S.; Brandt, U.; et al. Mitochondrial dysfunction, peroxidation damage and changes in glutathione metabolism in PARK6. Neurobiol. Dis. 2007, 25, 401-411. [CrossRef]

18. Parganlija, D.; Klinkenberg, M.; Domínguez-Bautista, J.; Hetzel, M.; Gispert, S.; Chimi, M.A.; Dröse, S.; Mai, S.; Brandt, U.; Auburger, G.; et al. Loss of PINK1 Impairs Stress-Induced Autophagy and Cell Survival. PLoS ONE 2014, 9, e95288. [CrossRef]

19. Klinkenberg, M.; Gispert, S.; Dominguez-Bautista, J.A.; Braun, I.; Auburger, G.; Jendrach, M. Restriction of trophic factors and nutrients induces PARKIN expression. Neurogenetics 2011, 13, 9-21. [CrossRef]

20. Gispert, S.; Ricciardi, F.; Kurz, A.; Azizov, M.; Hoepken, H.-H.; Becker, D.; Voos, W.; Leuner, K.; Müller, W.E.; Kudin, A.P.; et al. Parkinson Phenotype in Aged PINK1-Deficient Mice Is Accompanied by Progressive Mitochondrial Dysfunction in Absence of Neurodegeneration. PLoS ONE 2009, 4, e5777. [CrossRef]

21. Visanji, N.P.; Collingwood, J.F.; Finnegan, M.E.; Tandon, A.; House, E.; Hazrati, L.-N. Iron Deficiency in Parkinsonism: Region-Specific Iron Dysregulation in Parkinson's Disease and Multiple System Atrophy. J. Park. Dis. 2013, 3, 523-537. [CrossRef]

22. Ndayisaba, A.; Kaindlstorfer, C.; Wenning, G.K. Iron in Neurodegeneration-Cause or Consequence? Front. Neurosci. 2019, 13, 180. [CrossRef]

23. Wang, J.-Y.; Zhuang, Q.-Q.; Zhu, L.-B.; Zhu, H.; Li, T.; Li, R.; Chen, S.-F.; Huang, C.-P.; Zhang, X.; Zhu, J.-H. Meta-analysis of brain iron levels of Parkinson's disease patients determined by postmortem and MRI measurements. Sci. Rep. 2016, 6, 36669. [CrossRef]

24. Lei, P.; Ayton, S.; Finkelstein, D.I.; Spoerri, L.; Ciccotosto, G.D.; Wright, D.K.; Wong, B.X.; Adlard, P.A.; Cherny, R.A.; Lam, L.Q.; et al. Tau deficiency induces parkinsonism with dementia by impairing APP-mediated iron export. Nat. Med. 2012, 18, 291-295. [CrossRef] [PubMed]

25. Zecca, L.; Stroppolo, A.; Gatti, A.; Tampellini, D.; Toscani, M.; Gallorini, M.; Giaveri, G.; Arosio, P.; Santambrogio, P.; Fariello, R.G.; et al. The role of iron and copper molecules in the neuronal vulnerability of locus coeruleus and substantia nigra during aging. Proc. Natl. Acad. Sci. USA 2004, 101, 9843-9848. [CrossRef] [PubMed]

26. Hintze, K.J.; Theil, E.C. Cellular regulation and molecular interactions of the ferritins. Cell. Mol. Life Sci. 2006, 63, 591-600. [CrossRef] 
27. You, L.-H.; Li, Z.; Duan, X.; Zhao, B.-L.; Chang, S.-Y.; Shi, Z.-H. Mitochondrial ferritin suppresses MPTP-induced cell damage by regulating iron metabolism and attenuating oxidative stress. Brain Res. 2016, 1642, 33-42. [CrossRef]

28. Schweitzer, K.J.; Brüssel, T.; Leitner, P.; Krüger, R.; Bauer, P.; Woitalla, D.; Tomiuk, J.; Gasser, T.; Berg, D. Transcranial ultrasound in different monogenetic subtypes of Parkinson's disease. J. Neurol. 2007, 254, 613-616. [CrossRef]

29. Li, C.; Zhang, Y.; Cheng, X.; Yuan, H.; Zhu, S.; Liu, J.; Wen, Q.; Xie, Y.; Liu, J.; Kroemer, G.; et al. PINK1 and PARK2 Suppress Pancreatic Tumorigenesis through Control of Mitochondrial Iron-Mediated Immunometabolism. Dev. Cell 2018, 46, 441-455.e8. [CrossRef]

30. Kang, R.; Xie, Y.; Zeh, H.J.; Klionsky, D.J.; Tang, D. Mitochondrial quality control mediated by PINK1 and PRKN: Links to iron metabolism and tumor immunity. Autophagy 2018, 15, 172-173. [CrossRef]

31. Allen, G.F.; Toth, R.; James, J.; Gangley, I.G. Loss of iron triggers PINK1/Parkin-independent mitophagy. EMBO Rep. 2013, 14, 1127-1135. [CrossRef] [PubMed]

32. Esposito, G.; Vos, M.; Vilain, S.; Swerts, J.; Valadas, J.S.; Van Meensel, S.; Schaap, O.; Verstreken, P. Aconitase Causes Iron Toxicity in Drosophila pink1 Mutants. PLoS Genet. 2013, 9, e1003478. [CrossRef] [PubMed]

33. Auburger, G.; Gispert, S.; Brehm, N. Methyl-Arginine Profile of Brain from Aged PINK1-KO+A53T-SNCA Mice Suggests Altered Mitochondrial Biogenesis. Park. Dis. 2016, 2016, 1-13. [CrossRef] [PubMed]

34. Auburger, G.; Gispert, S.; Torres-Odio, S.; Jendrach, M.; Brehm, N.; Canet-Pons, J.; Key, J.; Sen, N.-E. SerThr-PhosphoProteome of Brain from Aged PINK1-KO+A53T-SNCA Mice Reveals pT1928-MAP1B and pS3781-ANK2 Deficits, as Hub between Autophagy and Synapse Changes. Int. J. Mol. Sci. 2019, 20, 3284. [CrossRef] [PubMed]

35. Torres-Odio, S.; Key, J.; Hoepken, H.-H.; Canet-Pons, J.; Valek, L.; Roller, B.; Walter, M.; Morales-Gordo, B.; Meierhofer, D.; Harter, P.N.; et al. Progression of pathology in PINK1-deficient mouse brain from splicing via ubiquitination, ER stress, and mitophagy changes to neuroinflammation. J. Neuroinflamm. 2017, 14, 1-26. [CrossRef] [PubMed]

36. Gispert, S.; Brehm, N.; Weil, J.; Seidel, K.; Rüb, U.; Kern, B.; Walter, M.; Roeper, J.; Auburger, G. Potentiation of neurotoxicity in double-mutant mice with Pink1 ablation and A53T-SNCA overexpression. Hum. Mol. Genet. 2015, 24, 1061-1076. [CrossRef]

37. Pickrell, A.M.; Huang, C.-H.; Kennedy, S.R.; Ordureau, A.; Sideris, D.P.; Hoekstra, J.G.; Harper, W.; Youle, R.J. Endogenous Parkin Preserves Dopaminergic Substantia Nigral Neurons following Mitochondrial DNA Mutagenic Stress. Neuron 2015, 87, 371-381. [CrossRef]

38. Sliter, D.A.; Martinez, J.; Hao, L.; Chen, X.; Sun, N.; Fischer, T.D.; Burman, J.L.; Li, Y.; Zhang, Z.; Narendra, D.P.; et al. Parkin and PINK1 mitigate STING-induced inflammation. Nature 2018, 561, 258-262. [CrossRef]

39. Matheoud, D.; Cannon, T.; Voisin, A.; Penttinen, A.-M.; Ramet, L.; Fahmy, A.M.; Ducrot, C.; Laplante, A.; Bourque, M.-J.; Zhu, L.; et al. Intestinal infection triggers Parkinson's disease-like symptoms in Pink1(-/-) mice. Nature 2019, 571, 565-569. [CrossRef]

40. Manzanillo, P.S.; Ayres, J.S.; Watson, R.O.; Collins, A.C.; Souza, G.; Rae, C.S.; Schneider, D.S.; Nakamura, K.; Shiloh, M.U.; Cox, J.S. The ubiquitin ligase parkin mediates resistance to intracellular pathogens. Nature 2013, 501, 512-516. [CrossRef]

41. Clark, I.E.; Dodson, M.W.; Jiang, C.; Cao, J.H.; Huh, J.R.; Seol, J.H.; Yoo, S.J.; Hay, B.A.; Guo, M. Drosophila pink1 is required for mitochondrial function and interacts genetically with parkin. Nature 2006, 441, 1162-1166. [CrossRef] [PubMed]

42. Saini, N.; Oelhafen, S.; Hua, H.; Georgiev, O.; Schaffner, W.; Büeler, H. Extended lifespan of Drosophila parkin mutants through sequestration of redox-active metals and enhancement of anti-oxidative pathways. Neurobiol. Dis. 2010, 40, 82-92. [CrossRef] [PubMed]

43. Cooper, J.F.; Machiela, E.; Dues, D.J.; Spielbauer, K.K.; Senchuk, M.M.; Van Raamsdonk, J.M. Activation of the mitochondrial unfolded protein response promotes longevity and dopamine neuron survival in Parkinson's disease models. Sci. Rep. 2017, 7, 16441. [CrossRef] [PubMed]

44. Nakamura, S.; Yoshimori, T. Autophagy and Longevity. Mol. Cells 2018, 41, 65-72. [PubMed]

45. Munkácsy, E.; Rea, S.L. The paradox of mitochondrial dysfunction and extended longevity. Exp. Gerontol. 2014, 56, 221-233. [CrossRef] [PubMed]

46. Butler, J.A.; Ventura, N.; Johnson, T.E.; Rea, S.L. Long-lived mitochondrial (Mit) mutants of Caenorhabditis elegans utilize a novel metabolism. FASEB J. 2010, 24, 4977-4988. [CrossRef] [PubMed] 
47. Fischer, F.; Weil, A.; Hamann, A.; Osiewacz, H.D. Human CLPP reverts the longevity phenotype of a fungal ClpP deletion strain. Nat. Commun. 2013, 4, 1397. [CrossRef]

48. Gispert, S.; Parganlija, D.; Klinkenberg, M.; Dröse, S.; Wittig, I.; Mittelbronn, M.; Grzmil, P.; Koob, S.; Hamann, A.; Walter, M.; et al. Loss of mitochondrial peptidase Clpp leads to infertility, hearing loss plus growth retardation via accumulation of CLPX, mtDNA and inflammatory factors. Hum. Mol. Genet. 2013, 22, 4871-4887. [CrossRef]

49. Bhaskaran, S.; Pharaoh, G.; Ranjit, R.; Murphy, A.; Matsuzaki, S.; Nair, B.C.; Forbes, B.; Gispert, S.; Auburger, G.; Humphries, K.M.; et al. Loss of mitochondrial protease ClpP protects mice from diet-induced obesity and insulin resistance. EMBO Rep. 2018, 19, e45009. [CrossRef]

50. Mai, S.; Klinkenberg, M.; Auburger, G.; Bereiter-Hahn, J.; Jendrach, M. Decreased expression of Drp1 and Fis1 mediates mitochondrial elongation in senescent cells and enhances resistance to oxidative stress through PINK1. J. Cell Sci. 2010, 123, 917-926. [CrossRef]

51. Seo, J.H.; Rivadeneira, D.B.; Caino, M.C.; Chae, Y.C.; Speicher, D.W.; Tang, H.-Y.; Vaira, V.; Bosari, S.; Palleschi, A.; Rampini, P.; et al. The Mitochondrial Unfoldase-Peptidase Complex ClpXP Controls Bioenergetics Stress and Metastasis. PLoS Biol. 2016, 14, e1002507. [CrossRef] [PubMed]

52. Alexeyev, M.F. Is there more to aging than mitochondrial DNA and reactive oxygen species? FEBS J. 2009, 276, 5768-5787. [CrossRef] [PubMed]

53. Hwang, A.B.; Jeong, D.-E.; Lee, S.-J.V. Mitochondria and Organismal Longevity. Curr. Genom. 2012, 13, 519-532. [CrossRef] [PubMed]

54. Huang, X.T.; Liu, X.; Ye, C.Y.; Tao, L.X.; Zhou, H.; Zhang, H.-Y. Iron-induced energy supply deficiency and mitochondrial fragmentation in neurons. J. Neurochem. 2018, 147, 816-830. [CrossRef] [PubMed]

55. Szklarczyk, D.; Gable, A.L.; Lyon, D.; Junge, A.; Wyder, S.; Huerta-Cepas, J.; Simonovic, M.; Doncheva, N.T.; Morris, J.H.; Bork, P.; et al. STRING v11: Protein-protein association networks with increased coverage, supporting functional discovery in genome-wide experimental datasets. Nucleic Acids Res. 2018, 47, D607-D613. [CrossRef]

56. Hernández-Gallardo, A.K.; Missirlis, F. Cellular iron sensing and regulation: Nuclear IRP1 extends a classic paradigm. Biochim. Biophys. Acta (BBA) 2020, 1867, 118705. [CrossRef]

57. Camaschella, C.; Campanella, A.; De Falco, L.; Boschetto, L.; Merlini, R.; Silvestri, L.; Levi, S.; Iolascon, A. The human counterpart of zebrafish shiraz shows sideroblastic-like microcytic anemia and iron overload. Blood 2007, 110, 1353-1358. [CrossRef]

58. Lipper, C.H.; Paddock, M.L.; Onuchic, J.N.; Mittler, R.; Nechushtai, R.; Jennings, P.A. Cancer-Related NEET Proteins Transfer 2Fe-2S Clusters to Anamorsin, a Protein Required for Cytosolic Iron-Sulfur Cluster Biogenesis. PLoS ONE 2015, 10, e0139699. [CrossRef]

59. Mons, C.; Ferecatu, I.; Riquier, S.; Lescop, E.; Bouton, C.; Golinelli, M.-P. Combined Biochemical, Biophysical, and Cellular Methods to Study Fe-S Cluster Transfer and Cytosolic Aconitase Repair by MitoNEET. Methods Enzymol. 2017, 595, 83-106. [CrossRef]

60. Kajarabille, N.; Latunde-Dada, G.O. Programmed Cell-Death by Ferroptosis: Antioxidants as Mitigators. Int. J. Mol. Sci. 2019, 20, 4968. [CrossRef]

61. Song, X.; Long, D. Nrf2 and Ferroptosis: A New Research Direction for Neurodegenerative Diseases. Front. Neurosci. 2020, 14, 267. [CrossRef] [PubMed]

62. Angelova, D.M.; Jones, H.B.L.; Brown, D.R. Levels of alpha- and beta-synuclein regulate cellular susceptibility to toxicity from alpha-synuclein oligomers. FASEB J. 2018, 32, 995-1006. [CrossRef]

63. Su, B.; Liu, H.; Wang, X.; Chen, S.G.; Siedlak, S.L.; Kondo, E.; Choi, R.; Takeda, A.; Castellani, R.J.; Perry, G.; et al. Ectopic localization of FOXO3a protein in Lewy bodies in Lewy body dementia and Parkinson's disease. Mol. Neurodegener. 2009, 4, 32. [CrossRef] [PubMed]

64. Beyer, K.; Ispierto, L.; Latorre, P.; Tolosa, E.; Ariza, A. Alpha- and beta-synuclein expression in Parkinson disease with and without dementia. J. Neurol. Sci. 2011, 310, 112-117. [CrossRef]

65. Brighina, L.; Okubadejo, N.U.; Schneider, N.K.; Lesnick, T.G.; De Andrade, M.; Cunningham, J.M.; Farrer, M.J.; Lincoln, S.J.; Rocca, W.A.; Maraganore, D.M. Beta-synuclein gene variants and Parkinson's disease: A preliminary case-control study. Neurosci. Lett. 2007, 420, 229-234. [CrossRef]

66. Davies, P.; Moualla, D.; Brown, D.R. Alpha-Synuclein Is a Cellular Ferrireductase. PLoS ONE 2011, 6, e15814. [CrossRef] 
67. Bhujabal, Z.; Birgisdottir, Å.B.; Sjøttem, E.; Brenne, H.B.; Øvervatn, A.; Habisov, S.; Kirkin, V.; Lamark, T.; Johansen, T. FKBP8 recruits LC3A to mediate Parkin-independent mitophagy. EMBO Rep. 2017, 18, 947-961. [CrossRef]

68. Liu, X.; Cheng, R.; Verbitsky, M.; Kisselev, S.; Browne, A.; Mejia-Sanatana, H.; Louis, E.D.; Cote, L.J.; Andrews, H.F.; Waters, C.H.; et al. Genome-Wide association study identifies candidate genes for Parkinson's disease in an Ashkenazi Jewish population. BMC Med Genet. 2011, 12, 104. [CrossRef] [PubMed]

69. Lahut, S.; Gispert, S.; Ömür, Ö.; Depboylu, C.; Seidel, K.; Domínguez-Bautista, J.A.; Brehm, N.; Tireli, H.; Hackmann, K.; Pirkevi, C.; et al. Blood RNA biomarkers in prodromal PARK4 and rapid eye movement sleep behavior disorder show role of complexin 1 loss for risk of Parkinson's disease. Dis. Model. Mech. 2017, 10, 619-631. [CrossRef]

70. Wu, Z.; Li, C.; Lv, S.; Zhou, B. Pantothenate kinase-associated neurodegeneration: Insights from a Drosophila model. Hum. Mol. Genet. 2009, 18, 3659-3672. [CrossRef]

71. Song, C.; Kanthasamy, A.G.; Anantharam, V.; Sun, F.; Kanthasamy, A.G. Environmental neurotoxic pesticide increases histone acetylation to promote apoptosis in dopaminergic neuronal cells: Relevance to epigenetic mechanisms of neurodegeneration. Mol. Pharmacol. 2010, 77, 621-632. [CrossRef] [PubMed]

72. Lane, D.J.R.; Merlot, A.; Huang, M.L.-H.; Bae, D.-H.; Jansson, P.J.; Sahni, S.; Kalinowski, D.S.; Richardson, D.R. Cellular iron uptake, trafficking and metabolism: Key molecules and mechanisms and their roles in disease. Biochim. Biophys. Acta (BBA) 2015, 1853, 1130-1144. [CrossRef] [PubMed]

73. Liu, Z.; Lanford, R.; Mueller, S.; Gerhard, G.S.; Luscieti, S.; Sanchez, M.; Devireddy, L. Siderophore-mediated iron trafficking in humans is regulated by iron. J. Mol. Med. 2012, 90, 1209-1221. [CrossRef] [PubMed]

74. Ren, Y.; Yang, S.; Tan, G.; Ye, W.; Liu, D.; Qian, X.; Ding, Z.; Zhong, Y.; Zhang, J.; Jiang, D.; et al. Reduction of Mitoferrin Results in Abnormal Development and Extended Lifespan in Caenorhabditis elegans. PLoS ONE 2012, 7, e29666. [CrossRef]

75. Yanatori, I.; Richardson, D.R.; Toyokuni, S.; Kishi, F. The iron chaperone poly(rC)-binding protein 2 forms a metabolon with the heme oxygenase $1 /$ cytochrome $\mathrm{P} 450$ reductase complex for heme catabolism and iron transfer. J. Biol. Chem. 2017, 292, 13205-13229. [CrossRef]

76. Leidgens, S.; Bullough, K.Z.; Shi, H.; Li, F.; Shakoury-Elizeh, M.; Yabe-Wada, T.; Subramanian, P.; Hsu, E.; Natarajan, N.; Nandal, A.; et al. Each Member of the Poly-r(C)-binding Protein 1 (PCBP) Family Exhibits Iron Chaperone Activity toward Ferritin. J. Biol. Chem. 2013, 288, 17791-17802. [CrossRef]

77. Galy, B.; Ferring-Appel, D.; Sauer, S.W.; Kaden, S.; Lyoumi, S.; Puy, H.; Kölker, S.; Gröne, H.-J.; Hentze, M.W. Iron Regulatory Proteins Secure Mitochondrial Iron Sufficiency and Function. Cell Metab. 2010, 12, $194-201$. [CrossRef]

78. Li, H.; Zhao, H.; Hao, S.; Shang, L.; Wu, J.; Song, C.; Meyron-Holtz, E.G.; Qiao, T.; Li, K. Iron regulatory protein deficiency compromises mitochondrial function in murine embryonic fibroblasts. Sci. Rep. 2018, 8, 5118. [CrossRef]

79. Chen, W.; Dailey, H.A.; Paw, B.H. Ferrochelatase forms an oligomeric complex with mitoferrin-1 and Abcb10 for erythroid heme biosynthesis. Blood 2010, 116, 628-630. [CrossRef]

80. Ichikawa, Y.; Bayeva, M.; Ghanefar, M.; Potini, V.; Sun, L.; Mutharasan, R.K.; Wu, R.; Khechaduri, A.; Naik, T.J.; Ardehali, H. Disruption of ATP-binding cassette B8 in mice leads to cardiomyopathy through a decrease in mitochondrial iron export. Proc. Natl. Acad. Sci. USA 2012, 109, 4152-4157. [CrossRef]

81. Pondarré, C.; Antiochos, B.B.; Campagna, D.R.; Clarke, S.L.; Greer, E.L.; Deck, K.M.; McDonald, A.; Han, A.-P.; Medlock, A.; Kutok, J.L.; et al. The mitochondrial ATP-binding cassette transporter Abcb7 is essential in mice and participates in cytosolic iron-sulfur cluster biogenesis. Hum. Mol. Genet. 2006, 15, 953-964. [CrossRef] [PubMed]

82. Lill, R.; Dutkiewicz, R.; Freibert, S.A.; Heidenreich, T.; Mascarenhas, J.; Netz, D.J.; Paul, V.D.; Pierik, A.J.; Richter, N.; Stümpfig, M.; et al. The role of mitochondria and the CIA machinery in the maturation of cytosolic and nuclear iron-sulfur proteins. Eur. J. Cell Biol. 2015, 94, 280-291. [CrossRef] [PubMed]

83. Maio, N.; Kim, K.S.; Holmes-Hampton, G.; Singh, A.; Rouault, T.A. Dimeric ferrochelatase bridges ABCB7 and $\mathrm{ABCB} 10$ homodimers in an architecturally defined molecular complex required for heme biosynthesis. Haematogica. 2019, 104, 1756-1767. [CrossRef] [PubMed]

84. Mühlenhoff, U.; Hoffmann, B.; Richter, N.; Rietzschel, N.; Spantgar, F.; Stehling, O.; Uzarska, M.A.; Lill, R. Compartmentalization of iron between mitochondria and the cytosol and its regulation. Eur. J. Cell Biol. 2015, 94, 292-308. [CrossRef] 
85. Maio, N.; Rouault, T.A. Mammalian Fe-S proteins: Definition of a consensus motif recognized by the co-chaperone HSC20. Metallomics 2016, 8, 1032-1046. [CrossRef]

86. Uzarska, M.A.; Nasta, V.; Weiler, B.D.; Spantgar, F.; Ciofi-Baffoni, S.; Saviello, M.R.; Gonnelli, L.; Mühlenhoff, U.; Banci, L.; Lill, R. Mitochondrial Bol1 and Bol3 function as assembly factors for specific iron-sulfur proteins. eLife 2016, 5, 83. [CrossRef]

87. Braymer, J.J.; Lill, R. Iron-sulfur cluster biogenesis and trafficking in mitochondria. J. Biol. Chem. 2017, 292, 12754-12763. [CrossRef]

88. Choi, A.M.; Alam, J. Heme oxygenase-1: Function, regulation, and implication of a novel stress-inducible protein in oxidant-induced lung injury. Am. J. Respir. Cell Mol. Biol. 1996, 15, 9-19. [CrossRef]

89. Mast, N.; Charvet, C.; Pikuleva, I.A.; Stout, C.D. Structural Basis of Drug Binding to CYP46A1, an Enzyme That Controls Cholesterol Turnover in the Brain. J. Biol. Chem. 2010, 285, 31783-31795. [CrossRef]

90. Correia, M.A.; Sinclair, P.R.; De Matteis, F. Cytochrome P450 regulation: The interplay between its heme and apoprotein moieties in synthesis, assembly, repair, and disposal. Drug Metab. Rev. 2011, 43, 1-26. [CrossRef]

91. De Sanctis, D.; Dewilde, S.; Pesce, A.; Moens, L.; Ascenzi, P.; Hankeln, T.; Burmester, T.; Bolognesi, M. Crystal Structure of Cytoglobin: The Fourth Globin Type Discovered in Man Displays Heme Hexa-coordination. J. Mol. Biol. 2004, 336, 917-927. [CrossRef] [PubMed]

92. Barthelme, M.; Scheele, U.; Dinkelaker, S.; Janoschka, A.; Macmillan, F.; Albers, S.-V.; Driessen, A.J.M.; Stagni, M.S.; Bill, E.; Meyer-Klaucke, W.; et al. Structural Organization of Essential Iron-Sulfur Clusters in the Evolutionarily Highly Conserved ATP-binding Cassette Protein ABCE1. J. Biol. Chem. 2007, 282, 14598-14607. [CrossRef] [PubMed]

93. Alhebshi, A.; Sideri, T.C.; Holland, S.L.; Avery, S.V. The essential iron-sulfur protein Rli1 is an important target accounting for inhibition of cell growth by reactive oxygen species. Mol. Biol. Cell 2012, 23, 3582-3590. [CrossRef] [PubMed]

94. Huang, B.; Johansson, M.J.; Byström, A.S. An early step in wobble uridine tRNA modification requires the Elongator complex. RNA 2005, 11, 424-436. [CrossRef] [PubMed]

95. Anderson, C.P.; Shen, M.; Eisenstein, R.S.; Leibold, E.A. Mammalian iron metabolism and its control by iron regulatory proteins. Biochim. Biophys. Acta (BBA) 2012, 1823, 1468-1483. [CrossRef] [PubMed]

96. Elbirt, K.K.; Bonkovsky, H.L. Heme oxygenase: Recent advances in understanding its regulation and role. Proc. Assoc. Am. Phys. 1999, 111, 438-447. [CrossRef]

97. Chung, J.; Anderson, S.; Gwynn, B.; Deck, K.M. Iron regulatory protein-1 protects against mitoferrin-1-deficient porphyria. J. Biol. Chem. 2014, 289, 7835-7843. [CrossRef]

98. Zhang, J.; Sun, X.; Wang, L.; Wong, Y.K.; Lee, Y.M.; Zhou, C.; Wu, G.; Zhao, T.; Yang, L.; Lu, L.; et al. Artesunate-induced mitophagy alters cellular redox status. Redox Biol. 2018, 19, 263-273. [CrossRef]

99. Salvador, G.A. Iron in neuronal function and dysfunction. BioFactors 2010, 36, 103-110. [CrossRef]

100. Hirsch, E.C. Altered regulation of iron transport and storage in Parkinson's disease. J. Neural. Transm. Suppl. 2006, 71, 201-204. [CrossRef]

101. Dexter, D.T.; Carayon, A.; Vidailhet, M.; Ruberg, M.; Agid, F.; Agid, Y.; Lees, A.J.; Wells, F.R.; Jenner, P.; Marsden, C.D. Decreased Ferritin Levels in Brain in Parkinson's Disease. J. Neurochem. 1990, 55, 16-20. [CrossRef] [PubMed]

102. Bauckman, K.A.; Haller, E.M.; Flores, I.; Nanjundan, M. Iron modulates cell survival in a Ras- and MAPK-dependent manner in ovarian cells. Cell Death Dis. 2013, 4, e592. [CrossRef] [PubMed]

103. Sheng, X.-J.; Tu, H.-J.; Chien, W.-L.; Kang, K.-H.; Lu, D.-H.; Liou, H.-H.; Lee, M.-J.; Fu, W.-M. Antagonism of proteasome inhibitor-induced heme oxygenase-1 expression by PINK1 mutation. PLoS ONE 2017, 12, e0183076. [CrossRef] [PubMed]

104. Durcan, T.M.; Fon, E.A. The three 'P's of mitophagy: PARKIN, PINK1, and post-translational modifications. Genes Dev. 2015, 29, 989-999. [CrossRef] [PubMed]

105. Requejo, R.; Lopez-Fabuel, I.; Fernandez, E.; Martins, L.M.; Almeida, A.; Bolaños, J.P. PINK1 deficiency sustains cell proliferation by reprogramming glucose metabolism through HIF1. Nat. Commun. 2014, 5, 4514. [CrossRef] [PubMed]

106. Murata, H.; Takamatsu, H.; Liu, S.; Kataoka, K.; Huh, N.-H.; Sakaguchi, M. NRF2 Regulates PINK1 Expression under Oxidative Stress Conditions. PLoS ONE 2015, 10, e0142438. [CrossRef] [PubMed] 
107. Liu, Y.; Yan, J.; Sun, C.; Li, G.; Li, S.; Zhang, L.; Di, C.; Gan, L.; Wang, Y.; Zhou, R.; et al. Ameliorating mitochondrial dysfunction restores carbon ion-induced cognitive deficits via co-activation of NRF2 and PINK1 signaling pathway. Redox Biol. 2018, 17, 143-157. [CrossRef]

108. Chen, X.-L.; Kunsch, C. Induction of cytoprotective genes through Nrf2/antioxidant response element pathway: A new therapeutic approach for the treatment of inflammatory diseases. Curr. Pharm. Des. 2004, 10, 879-891. [CrossRef]

109. Kerins, M.J.; Ooi, A. The Roles of NRF2 in Modulating Cellular Iron Homeostasis. Antioxidants Redox Signal. 2018, 29, 1756-1773. [CrossRef]

110. Lau, A.; Wang, X.-J.; Zhao, F.; Villeneuve, N.F.; Wu, T.; Jiang, T.; Sun, Z.; White, E.; Zhang, D.D. A Noncanonical Mechanism of Nrf2 Activation by Autophagy Deficiency: Direct Interaction between Keap1 and p62. Mol. Cell. Biol. 2010, 30, 3275-3285. [CrossRef]

111. Zhang, Y.; Xiang, Y. Molecular and cellular basis for the unique functioning of Nrf1, an indispensable transcription factor for maintaining cell homoeostasis and organ integrity. Biochem. J. 2016, 473, 961-1000. [CrossRef] [PubMed]

112. Kaelin, W.G. Proline hydroxylation and gene expression. Annu. Rev. Biochem. 2005, 74, 115-128. [CrossRef]

113. Lill, R.; Freibert, S.-A. Mechanisms of Mitochondrial Iron-Sulfur Protein Biogenesis. Annu. Rev. Biochem. 2020, 89, 471-499. [CrossRef] [PubMed]

114. Huynh, N.; Ou, Q.; Cox, P.; Lill, R.; King-Jones, K. Glycogen branching enzyme controls cellular iron homeostasis via Iron Regulatory Protein 1 and mitoNEET. Nat. Commun. 2019, 10,1-18. [CrossRef]

115. Yuan, H.; Li, X.; Zhang, X.; Kang, R.; Tang, D. CISD1 inhibits ferroptosis by protection against mitochondrial lipid peroxidation. Biochem. Biophys. Res. Commun. 2016, 478, 838-844. [CrossRef] [PubMed]

116. Kessler, K.R.; Hamscho, N.; Morales, B.; Menzel, C.; Barrero, F.; Vives, F.; Gispert, S.; Auburger, G. Dopaminergic function in a family with the PARK6 form of autosomal recessive Parkinson's syndrome. J. Neural Transm. 2005, 112, 1345-1353. [CrossRef] [PubMed]

117. Tuin, I.; Voss, U.; Kessler, K.; Krakow, K.; Hilker, R.; Morales, B.; Steinmetz, H.; Auburger, G. Sleep quality in a family with hereditary parkinsonism (PARK6). Sleep Med. 2008, 9, 684-688. [CrossRef]

118. Hoepken, H.-H.; Gispert, S.; Azizov, M.; Klinkenberg, M.; Ricciardi, F.; Kurz, A.; Morales-Gordo, B.; Bonin, M.; Riess, O.; Gasser, T.; et al. Parkinson patient fibroblasts show increased alpha-synuclein expression. Exp. Neurol. 2008, 212, 307-313. [CrossRef]

119. Klinkenberg, M.; Thurow, N.; Gispert, S.; Ricciardi, F.; Eich, F.; Prehn, J.H.M.; Auburger, G.; Kögel, D. Enhanced vulnerability of PARK6 patient skin fibroblasts to apoptosis induced by proteasomal stress. Neuroscience 2010, 166, 422-434. [CrossRef]

120. Auburger, G.; Klinkenberg, M.; Drost, J.; Marcus, K.; Morales-Gordo, B.; Kunz, W.S.; Brandt, U.; Broccoli, V.; Reichmann, H.; Gispert, S.; et al. Primary Skin Fibroblasts as a Model of Parkinson's Disease. Mol. Neurobiol. 2012, 46, 20-27. [CrossRef]

121. Gehrke, S.; Wu, Z.; Klinkenberg, M.; Sun, Y.; Auburger, G.; Guo, S.; Lu, B. PINK1 and Parkin control localized translation of respiratory chain component mRNAs on mitochondria outer membrane. Cell Metab. 2015, 21, 95-108. [CrossRef]

122. Azkona, G.; De Maturana, R.L.; Del Rio, P.; Sousa, A.; Vazquez, N.; Zubiarrain, A.; Jimenez-Blasco, D.; Bolaños, J.P.; Morales, B.; Auburger, G.; et al. LRRK2 Expression Is Deregulated in Fibroblasts and Neurons from Parkinson Patients with Mutations in PINK1. Mol. Neurobiol. 2018, 55, 506-516. [CrossRef] [PubMed]

123. Wu, Z.; Tantray, I.; Lim, J.; Chen, S.; Li, Y.; Davis, Z.; Sitron, C.; Dong, J.; Gispert, S.; Auburger, G.; et al. MISTERMINATE Mechanistically Links Mitochondrial Dysfunction with Proteostasis Failure. Mol. Cell 2019, 75, 835-848.e8. [CrossRef] [PubMed]

124. Brown, D.R. alpha-Synuclein as a ferrireductase. Biochem. Soc. Trans. 2013, 41, 1513-1517. [PubMed]

125. McDowall, J.S.; Ntai, I.; Honeychurch, K.C.; Hart, J.P.; Colin, P.; Schneider, B.; Brown, D.R. Alpha-synuclein ferrireductase activity is detectible in vivo, is altered in Parkinson's disease and increases the neurotoxicity of DOPAL. Mol. Cell. Neurosci. 2017, 85, 1-11. [CrossRef] [PubMed]

126. McDowall, J.S.; Brown, D.R. Alpha-synuclein: Relating metals to structure, function and inhibition. Metallomics 2016, 8, 385-397. [CrossRef] [PubMed]

127. McLeary, F.; Rcom-H'Cheo-Gauthier, A.N.; Goulding, M.; Radford, R.; Okita, Y.; Faller, P.; Chung, R.S.; Pountney, D.L. Switching on Endogenous Metal Binding Proteins in Parkinson's Disease. Cells 2019, 8, 179. [CrossRef] 
128. Roberts, H.L.; Schneider, B.L.; Brown, D.R. alpha-Synuclein increases beta-amyloid secretion by promoting beta-/gamma-secretase processing of APP. PLoS ONE 2017, 12, e0171925.

129. Guardia-Laguarta, C.; Area-Gomez, E.; Schon, E.A.; Przedborski, S. A new role for $\alpha$-synuclein in Parkinson's disease: Alteration of ER-mitochondrial communication. Mov. Disord. 2015, 30, 1026-1033. [CrossRef]

130. Wolozin, B.; Golts, N. Book Review: Iron and Parkinson's Disease. Neuroscientist 2002, 8, 22-32. [CrossRef]

131. Chen, B.; Wen, X.; Jiang, H.; Wang, J.; Song, N.; Xie, J. Interactions between iron and $\alpha$-synuclein pathology in Parkinson's disease. Free Radic. Biol. Med. 2019, 141, 253-260. [CrossRef] [PubMed]

132. Furukawa, T.; Naitoh, Y.; Kohno, H.; Tokunaga, R.; Taketani, S. Iron deprivation decreases ribonucleotide reductase activity and DNA synthesis. Life Sci. 1992, 50, 2059-2065. [CrossRef] [PubMed]

133. Walter, P.B.; Knutson, M.D.; Paler-Martinez, A.; Lee, S.; Xu, Y.; Viteri, F.E.; Ames, B.N. Iron deficiency and iron excess damage mitochondria and mitochondrial DNA in rats. Proc. Natl. Acad. Sci. USA 2002, 99, 2264-2269. [CrossRef] [PubMed]

134. Bender, A.; Krishnan, K.J.; Morris, C.M.; Taylor, G.A.; Reeve, A.K.; Perry, R.H.; Jaros, E.; Hersheson, J.S.; Betts, J.; Klopstock, T.; et al. High levels of mitochondrial DNA deletions in substantia nigra neurons in aging and Parkinson disease. Nat. Genet. 2006, 38, 515-517. [CrossRef] [PubMed]

135. Erlitzki, R.; Long, J.C.; Theil, E.C. Multiple, conserved iron-responsive elements in the $3^{\prime}$-untranslated region of transferrin receptor mRNA enhance binding of iron regulatory protein 2. J. Biol. Chem. 2002, 277, 42579-42587.

136. Li, J.; Braganza, A.; Sobol, R.W. Base Excision Repair Facilitates a Functional Relationship Between Guanine Oxidation and Histone Demethylation. Antioxid. Redox Signal. 2013, 18, 2429-2443. [CrossRef]

137. Martelli, A.; Wattenhofer-Donzé, M.; Schmucker, S.; Bouvet, S.; Reutenauer, L.; Puccio, H. Frataxin is essential for extramitochondrial Fe-S cluster proteins in mammalian tissues. Hum. Mol. Genet. 2007, 16, 2651-2658. [CrossRef]

138. Vaubel, R.A.; Isaya, G. Iron-sulfur cluster synthesis, iron homeostasis and oxidative stress in Friedreich ataxia. Mol. Cell. Neurosci. 2013, 55, 50-61. [CrossRef]

139. Crooks, D.R.; Maio, N.; Lane, A.N.; Jarnik, M.; Higashi, R.M.; Haller, R.G.; Yang, Y.; Fan, T.W.-M.; Linehan, W.M.; Rouault, T.A. Acute loss of iron-sulfur clusters results in metabolic reprogramming and generation of lipid droplets in mammalian cells. J. Biol. Chem. 2018, 293, 8297-8311. [CrossRef]

140. Haskamp, V.; Karrie, S.; Mingers, T.; Barthels, S.; Alberge, F.; Magalon, A.; Müller, K.; Bill, E.; Lubitz, W.; Kleeberg, K.; et al. The radical SAM protein HemW is a heme chaperone. J. Biol. Chem. 2017, 293, 2558-2572. [CrossRef]

141. Latorre-Pellicer, A.; Moreno-Loshuertos, R.; Lechuga-Vieco, A.V.; Sánchez-Cabo, F.; Torroja, C.; Acín-Pérez, R.; Calvo, E.; Aix, E.; González-Guerra, A.; Logan, A.; et al. Mitochondrial and nuclear DNA matching shapes metabolism and healthy ageing. Nature 2016, 535, 561-565. [CrossRef]

142. Genetic Modifiers of Huntington's Disease (GeM-HD) Consortium. Identification of Genetic Factors that Modify Clinical Onset of Huntington's Disease. Cell 2015, 162, 516-526.

143. Jones, L.; Houlden, H.; Tabrizi, S.J. DNA repair in the trinucleotide repeat disorders. Lancet Neurol. 2017, 16, 88-96. [CrossRef] [PubMed]

144. McKinnon, P.J.; Caldecott, K.W. DNA Strand Break Repair and Human Genetic Disease. Annu. Rev. Genom. Hum. Genet. 2007, 8, 37-55. [CrossRef]

145. Piel, R.B.; Shiferaw, M.T.; Vashisht, A.A.; Marcero, J.R.; Praissman, J.L.; Phillips, J.D.; Wohlschlegel, J.A.; Medlock, A. A Novel Role for Progesterone Receptor Membrane Component 1 (PGRMC1): A Partner and Regulator of Ferrochelatase. Biochemistry 2016, 55, 5204-5217. [CrossRef] [PubMed]

146. Choi, J.W.; Kim, S.K.; Pai, S.H. Changes in serum lipid concentrations during iron depletion and after iron supplementation. Ann. Clin. Lab. Sci. 2001, 31, 151-156.

147. Llorens, J.V.; Metzendorf, C.; Missirlis, F.; Lind, M.I. Mitochondrial iron supply is required for the developmental pulse of ecdysone biosynthesis that initiates metamorphosis in Drosophila melanogaster. JBIC J. Biol. Inorg. Chem. 2015, 20, 1229-1238. [CrossRef]

148. Wu, Z.; Wang, Y.; Lim, J.; Liu, B.; Li, Y.; Vartak, R.; Stankiewicz, T.; Montgomery, S.; Lu, B. Ubiquitination of ABCE1 by NOT4 in Response to Mitochondrial Damage Links Co-translational Quality Control to PINK1-Directed Mitophagy. Cell Metab. 2018, 28, 130-144.

149. Mehta, R.; Chandler-Brown, D.; Ramos, F.J.; Shamieh, L.S.; Kaeberlein, M. Regulation of mRNA Translation as a Conserved Mechanism of Longevity Control. Adv. Exp. Med. Biol. 2010, 694, 14-29. [CrossRef] 
Cells 2020, 9, 2229

150. MacInnes, A.W. The role of the ribosome in the regulation of longevity and lifespan extension. Wiley Interdiscip. Rev. RNA 2016, 7, 198-212. [CrossRef]

151. Coelho, C.M.A.; Kolevski, B.; Bunsn, C.; Walker, C.; Dahanukar, A.; Leevers, S.J. Growth and cell survival are unevenly impaired in pixie mutant wing discs. Development 2005, 132, 5411-5424. [CrossRef] [PubMed]

152. Nürenberg-Goloub, E.; Heinemann, H.; Gerovac, M.; Tampé, R. Ribosome recycling is coordinated by processive events in two asymmetric ATP sites of ABCE1. Life Sci. Alliance 2018, 1, e201800095. [CrossRef] [PubMed]

153. Sudmant, P.H.; Lee, H.; Dominguez, D.; Heiman, M.; Burge, C.B. Widespread Accumulation of Ribosome-Associated Isolated 3' UTRs in Neuronal Cell Populations of the Aging Brain. Cell Rep. 2018, 25, 2447-2456.e4. [PubMed]

154. Le Roy, F.; Bisbal, C.; Silhol, M.; Martinand, C.; Lebleu, B.; Salehzada, T. The 2-5A/RNase L/RNase L inhibitor (RLI) [correction of (RNI)] pathway regulates mitochondrial mRNAs stability in interferon alpha-treated H9 cells. J. Biol. Chem. 2001, 276, 48473-48482.

155. Stadhouders, R.; Aktuna, S.; Thongjuea, S.; Aghajanirefah, A.; Pourfarzad, F.; Van Ijcken, W.; Lenhard, B.; Rooks, H.; Best, S.; Menzel, S.; et al. HBS1L-MYB intergenic variants modulate fetal hemoglobin via long-range MYB enhancers. J. Clin. Investig. 2014, 124, 1699-1710. [CrossRef]

156. Volani, C.; Paglia, G.; Smarason, S.V.; Pramstaller, P.P.; Demetz, E.; Pfeifhofer-Obermair, C.; Weiss, G. Metabolic Signature of Dietary Iron Overload in a Mouse Model. Cells 2018, 7, 264. [CrossRef]

157. Finazzi, D.; Arosio, P. Biology of ferritin in mammals: An update on iron storage, oxidative damage and neurodegeneration. Arch. Toxicol. 2014, 88, 1787-1802. [CrossRef]

158. Yévenes, A. The Ferritin Superfamily. Plant Microbe Interact. 2017, 83, 75-102. [CrossRef]

159. Geldenhuys, W.J.; Benkovic, S.A.; Lin, L.; Yonutas, H.M.; Crish, S.D.; Sullivan, P.G.; Darvesh, A.S.; Brown, C.M.; Richardson, J.R. MitoNEET (CISD1) Knockout Mice Show Signs of Striatal Mitochondrial Dysfunction and a Parkinson's Disease Phenotype. ACS Chem. Neurosci. 2017, 8, 2759-2765. [CrossRef]

160. Lee, J.; You, J.H.; Shin, D.; Roh, J.-L. Inhibition of Glutaredoxin 5 predisposes Cisplatin-resistant Head and Neck Cancer Cells to Ferroptosis. Theranostics 2020, 10, 7775-7786. [CrossRef]

161. Chi, H.; Tang, W.; Bai, Y. Molecular evidence of impaired iron metabolism and its association with Parkinson's disease progression. 3 Biotech 2020, 10, 173-177. [CrossRef] [PubMed]

162. Faucheux, B.A.; Martin, M.-E.; Beaumont, C.; Hunot, S.; Hauw, J.-J.; Agid, Y.; Hirsch, E.C. Lack of up-regulation of ferritin is associated with sustained iron regulatory protein-1 binding activity in the substantia nigra of patients with Parkinson's disease. J. Neurochem. 2002, 83, 320-330. [CrossRef] [PubMed]

163. Salazar, J.; Mena, N.; Núñez, M.T. Iron dyshomeostasis in Parkinson's disease. J. Neural Transm. Suppl. 2006, 71, 205-213. [CrossRef]

164. Mena, N.P.; Bulteau, A.-L.; Salazar, J.; Hirsch, E.C.; Núñez, M.T. Effect of mitochondrial complex I inhibition on Fe-S cluster protein activity. Biochem. Biophys. Res. Commun. 2011, 409, 241-246. [CrossRef] [PubMed]

165. Yu, X.; Song, N.; Guo, X.; Jiang, H.; Zhang, H.; Xie, J. Differences in vulnerability of neurons and astrocytes to heme oxygenase-1 modulation: Implications for mitochondrial ferritin. Sci. Rep. 2016, 6, 24200. [CrossRef] [PubMed]

166. Hirose, W.; Ikematsu, K.; Tsuda, R. Age-associated increases in heme oxygenase-1 and ferritin immunoreactivity in the autopsied brain. Leg. Med. 2003, 5 (Suppl. 1), S360-S366. [CrossRef]

167. Galazka-Friedman, J.; Bauminger, E.R.; Koziorowski, D.; Friedman, A. Mössbauer spectroscopy and ELISA studies reveal differences between Parkinson's disease and control substantia nigra. Biochim. Biophys. Acta (BBA) 2004, 1688, 130-136. [CrossRef]

168. Wypijewska, A.; Galazka-Friedman, J.; Bauminger, E.R.; Wszolek, Z.K.; Schweitzer, K.J.; Dickson, D.W.; Jaklewicz, A.; Elbaum, D.; Friedman, A. Iron and reactive oxygen species activity in parkinsonian substantia nigra. Park. Relat. Disord. 2010, 16, 329-333. [CrossRef]

169. Huo, L.-R.; Shen, C.; Ju, W.; Zou, J.-H.; Yan, W.; Brown, W.T.; Zhong, N. Identification of novel partner proteins of PCBP1. J. Peking Univ. Heal. Sci. 2009, 41, 402-408.

170. Shi, H.; Bencze, K.Z.; Stemmler, T.L.; Philpott, C.C. A Cytosolic Iron Chaperone That Delivers Iron to Ferritin. Science 2008, 320, 1207-1210. [CrossRef]

171. Henn, I.H.; Bouman, B.; Schlehe, J.S.; Schlierf, A.; Schramm, J.E.; Wegener, E.; Nakaso, K.; Culmsee, C.; Berninger, B.; Krappmann, D.; et al. Parkin mediates neuroprotection through activation of IkappaB kinase/nuclear factor-kappaB signaling. J. Neurosci. 2007, 27, 1868-1878. [PubMed] 
Cells 2020, 9, 2229

172. Williams, N.C.; O'Neill, L.A. A Role for the Krebs Cycle Intermediate Citrate in Metabolic Reprogramming in Innate Immunity and Inflammation. Front. Immunol. 2018, 9, 141. [CrossRef] [PubMed]

173. Chen-Roetling, J.; Liu, W.; Regan, R.F. Iron accumulation and neurotoxicity in cortical cultures treated with holotransferrin. Free Radic. Biol. Med. 2011, 51, 1966-1974. [CrossRef] [PubMed]

174. Avila, F.; Echeverría, G.; Pérez, D.; Martinez, C.; Strobel, P.; Castillo, O.; Villaroel, L.; Mezzano, D.; Rozowski, J.; Urquiaga, I.; et al. Serum Ferritin Is Associated with Metabolic Syndrome and Red Meat Consumption. Oxid. Med. Cell. Longev. 2015, 2015. [CrossRef]

175. Lane, D.J.R.; Richardson, D.R. The active role of vitamin C in mammalian iron metabolism: Much more than just enhanced iron absorption! Free Radic. Biol. Med. 2014, 75, 69-83. [CrossRef]

176. Reilly, D.K.; Hershey, L.; Rivera-Calimlim, L.; Shoulson, I. On-off effects in Parkinson's disease: A controlled investigation of ascorbic acid therapy. Adv. Neurol. 1983, 37, 51-60.

177. Yapa, S.S. Detection of subclinical ascorbate deficiency in early Parkinson's disease. Public Health 1992, 106, 393-395. [CrossRef]

178. Fahn, S. A pilot trial of high-dose alpha-tocopherol and ascorbate in early Parkinson's disease. Ann. Neurol. 1992, 32, S128-S132. [CrossRef]

179. Logroscino, G.; Gao, X.; Chen, H.; Wing, A.; Ascherio, A. Dietary Iron Intake and Risk of Parkinson's Disease. Am. J. Epidemiol. 2008, 168, 1381-1388. [CrossRef]

180. Yang, F.; Wolk, A.; Håkansson, N.; Pedersen, N.L.; Wirdefeldt, K. Dietary antioxidants and risk of Parkinson's disease in two population-based cohorts. Mov. Disord. 2017, 32, 1631-1636. [CrossRef]

181. Wan, Z.; Xu, J.; Huang, Y.; Zhai, Y.; Ma, Z.; Zhou, B.; Cao, Z. Elevating bioavailable iron levels in mitochondria suppresses the defective phenotypes caused by PINK1 loss-of-function in Drosophila melanogaster. Biochem. Biophys. Res. Commun. 2020. [CrossRef]

182. Seguin, A.; Takahashi-Makise, N.; Yien, Y.Y.; Huston, N.C.; Whitman, J.C.; Musso, G.; Wallace, J.A.; Bradley, T.; Bergonia, H.A.; Kafina, M.D.; et al. Reductions in the mitochondrial ABC transporter Abcb10 affect the transcriptional profile of heme biosynthesis genes. J. Biol. Chem. 2017, 292, 16284-16299. [CrossRef] [PubMed]

183. Chen, W.; Paradkar, P.N.; Li, L.; Pierce, E.L.; Langer, N.B.; Takahashi-Makise, N.; Hyde, B.B.; Shirihai, O.S.; Ward, D.M.; Kaplan, J.; et al. Abcb10 physically interacts with mitoferrin-1 (Slc25a37) to enhance its stability and function in the erythroid mitochondria. Proc. Natl. Acad. Sci. USA 2009, 106, 16263-16268. [CrossRef] [PubMed]

184. Shaw, G.C.; Cope, J.J.; Li, L.; Corson, K.; Hersey, C.; Ackermann, G.E.; Gwynn, B.; Lambert, A.J.; Wingert, R.A.; Traver, D.; et al. Mitoferrin is essential for erythroid iron assimilation. Nature 2006, 440, 96-100. [CrossRef] [PubMed]

185. Paradkar, P.N.; Zumbrennen, K.B.; Paw, B.H.; Ward, D.M.; Kaplan, J. Regulation of Mitochondrial Iron Import through Differential Turnover of Mitoferrin 1 and Mitoferrin 2. Mol. Cell. Biol. 2008, 29, 1007-1016. [CrossRef] [PubMed]

186. Schmittgen, T.D.; Livak, K.J. Analyzing real-time PCR data by the comparative CT method. Nat. Protoc. 2008, 3, 1101-1108. [CrossRef]

187. Sen, N.-E.; Canet-Pons, J.; Halbach, M.V.; Arsovic, A.; Pilatus, U.; Chae, W.-H.; Kaya, Z.-E.; Seidel, K.; Rollmann, E.; Mittelbronn, M.; et al. Generation of an Atxn2-CAG100 knock-in mouse reveals $\mathrm{N}$-acetylaspartate production deficit due to early Nat81 dysregulation. Neurobiol. Dis. 2019, 132, 104559. [CrossRef]

188. Kulak, N.A.; Pichler, G.; Paron, I.; Nagaraj, N.; Mann, M. Minimal, encapsulated proteomic-sample processing applied to copy-number estimation in eukaryotic cells. Nat. Methods 2014, 11, 319-324. [CrossRef]

189. Gielisch, I.; Meierhofer, D. Metabolome and Proteome Profiling of Complex I Deficiency Induced by Rotenone. J. Proteome Res. 2014, 14, 224-235. [CrossRef]

(C) 2020 by the authors. Licensee MDPI, Basel, Switzerland. This article is an open access article distributed under the terms and conditions of the Creative Commons Attribution (CC BY) license (http://creativecommons.org/licenses/by/4.0/). 


\subsection{Publication III}

\section{Declaration of author contributions}

Title: Ubiquitylome profiling of Parkin-null brain reveals dysregulation of calcium homeostasis factors ATP1A2, Hippocalcin and GNA11, reflected by altered firing of noradrenergic neurons Status: published

Journal: Neurobiology of Disease, 2019, doi: 10.1016/j.nbd.2019.02.008

Contributing authors: Key J. (JK)*, Mueller A.K. (AKM)*, Gispert S. (SG), Matschke L. (LM), Wittig I. (IW), Corti O. (OG), Münch C. (CM), Decher N. (ND), Auburger G. (GA)

* shared first authorship

What are the contributions of the doctoral candidate and her co-authors?

\section{(1) Concept and design}

Doctoral candidate (JK): $60 \%$

Co-author AKM: $20 \%$

Co-author GA: $\mathbf{2 0 \%}$

\section{(2) Conducting tests and experiments}

Doctoral candidate (JK): $\mathbf{5 5 \%}$ (expression analyses, immunostaining)

Co-author AKM: $\mathbf{3 0 \%}$ (electrophysiological experiments)

Co-author LM: 15\% (electrophysiological experiments)

\section{(3) Compilation of data sets and figures}

Doctoral candidate (JK): $\mathbf{6 0 \%}$ (expression analyses, immunostaining)

Co-author AKM: 25\% (electrophysiological data)

Co-author LM: $\mathbf{5 \%}$ (electrophysiological data)

Co-author ND: $\mathbf{5 \%}$ (electrophysiological data)

Co-author GA: $\mathbf{5 \%}$ (ubiquitylome)

\section{(4) Analysis and interpretation of data}

Doctoral candidate (JK): $\mathbf{5 5 \%}$ (expression analyses, immunostaining)

Co-author AKM: $20 \%$ (electrophysiological data)

Co-author LM: $\mathbf{1 0 \%}$ (electrophysiological data)

Co-author ND: 5\% (electrophysiological data)

Co-author GA: 10\% (Interpretation of all data)

\section{(5) Drafting of manuscript}

Doctoral candidate (JK): $\mathbf{6 0 \%}$

Co-author AKM: $15 \%$

Co-author LM: $10 \%$

Co-author ND: $\mathbf{5 \%}$

Co-author GA: $10 \%$

Date and place

Signature of the doctoral candidate

Date and place

Signature of the supervisor 


\title{
Ubiquitylome profiling of Parkin-null brain reveals dysregulation of calcium homeostasis factors ATP1A2, Hippocalcin and GNA11, reflected by altered firing of noradrenergic neurons
}

\author{
Key J. ${ }^{\mathrm{a}, 1}$, Mueller A.K. ${ }^{\mathrm{b}, 1}$, Gispert S. ${ }^{\mathrm{a}}$, Matschke L. ${ }^{\mathrm{b}}$, Wittig I. ${ }^{\mathrm{c}}$, Corti O. ${ }^{\mathrm{d}, \mathrm{e}, \mathrm{f}, \mathrm{g}}$, Münch C. ${ }^{\mathrm{h}}$, \\ Decher N. ${ }^{\text {b,*, }}$, Auburger G. ${ }^{\text {a, }}$ \\ ${ }^{\text {a }}$ Exp. Neurology, Goethe University Medical School, 60590 Frankfurt am Main, Germany \\ ${ }^{\mathrm{b}}$ Institute for Physiology and Pathophysiology, Vegetative Physiology and Marburg Center for Mind, Brain and Behavior - MCMBB; Clinic for Neurology, Philipps-University \\ Marburg, 35037 Marburg, Germany \\ ${ }^{\mathrm{c}}$ Functional Proteomics, SFB 815 Core Unit, Goethe University Medical School, 60590 Frankfurt am Main, Germany \\ d Institut du Cerveau et de la Moelle épinière, ICM, Paris, F-75013, France \\ e Inserm, U1127, Paris, F-75013, France \\ ${ }^{\mathrm{f}}$ CNRS, UMR 7225, Paris, F-75013, France \\ ${ }^{\mathrm{g}}$ Sorbonne Universités, Paris, F-75013, France \\ ${ }^{\mathrm{h}}$ Institute of Biochemistry II, Goethe University Medical School, 60590 Frankfurt am Main, Germany
}

\section{A R T I C L E I N F O}

\section{Keywords:}

Parkinson's disease

Mitochondria

Parkin

Ubiquitin

Calcium

Firing frequency

\begin{abstract}
A B S T R A C T
Parkinson's disease (PD) is the second most frequent neurodegenerative disorder in the old population. Among its monogenic variants, a frequent cause is a mutation in the Parkin gene (Prkn). Deficient function of Parkin triggers ubiquitous mitochondrial dysfunction and inflammation in the brain, but it remains unclear how selective neural circuits become vulnerable and finally undergo atrophy.

We attempted to go beyond previous work, mostly done in peripheral tumor cells, which identified protein targets of Parkin activity, an ubiquitin E3 ligase. Thus, we now used aged Parkin-knockout (KO) mouse brain for a global quantification of ubiquitylated peptides by mass spectrometry (MS). This approach confirmed the most abundant substrate to be VDAC3, a mitochondrial outer membrane porin that modulates calcium flux, while uncovering also $>3$-fold dysregulations for neuron-specific factors. Ubiquitylation decreases were prominent for Hippocalcin (HPCA), Calmodulin (CALM1/CALML3), Pyruvate Kinase (PKM2), sodium/potassium-transporting ATPases (ATP1A1/2/3/4), the Rab27A-GTPase activating protein alpha (TBC1D10A) and an ubiquitin ligase adapter (DDB1), while strong increases occurred for calcium transporter ATP2C1 and G-protein subunits G(i)/ $\mathrm{G}(\mathrm{o}) / \mathrm{G}(\mathrm{Tr})$. Quantitative immunoblots validated elevated abundance for the electrogenic pump ATP1A2, for HPCA as neuron-specific calcium sensor, which stimulates guanylate cyclases and modifies axonal slow afterhyperpolarization (sAHP), and for the calcium-sensing G-protein GNA11. We assessed if compensatory molecular regulations become insufficient over time, leading to functional deficits. Patch clamp experiments in acute Parkin-KO brain slices indeed revealed alterations of the electrophysiological properties in aged noradrenergic locus coeruleus (LC) neurons. LC neurons of aged Parkin-KO brain showed an acceleration of the spontaneous pacemaker frequency, a reduction in SAHP and shortening of action potential duration, without modulation of KCNQ potassium currents.

These findings indicate altered calcium-dependent excitability in a PARK2 model of PD, mediated by diminished turnover of potential Parkin targets such as ATP1A2 and HPCA. The data also identified further novel Parkin substrate candidates like SIRT2, OTUD7B and CUL5. Our elucidation of neuron-specific mechanisms of PD pathogenesis helps to explain the known exceptional susceptibility of noradrenergic and dopaminergic projections to alterations of calcium homeostasis and its mitochondrial buffering.
\end{abstract}

\footnotetext{
* Corresponding authors.

E-mail addresses: decher@staff.uni-marburg.de (N. Decher), auburger@em.uni-frankfurt.de (G. Auburger).

${ }^{1}$ Joint first authorship.
} 

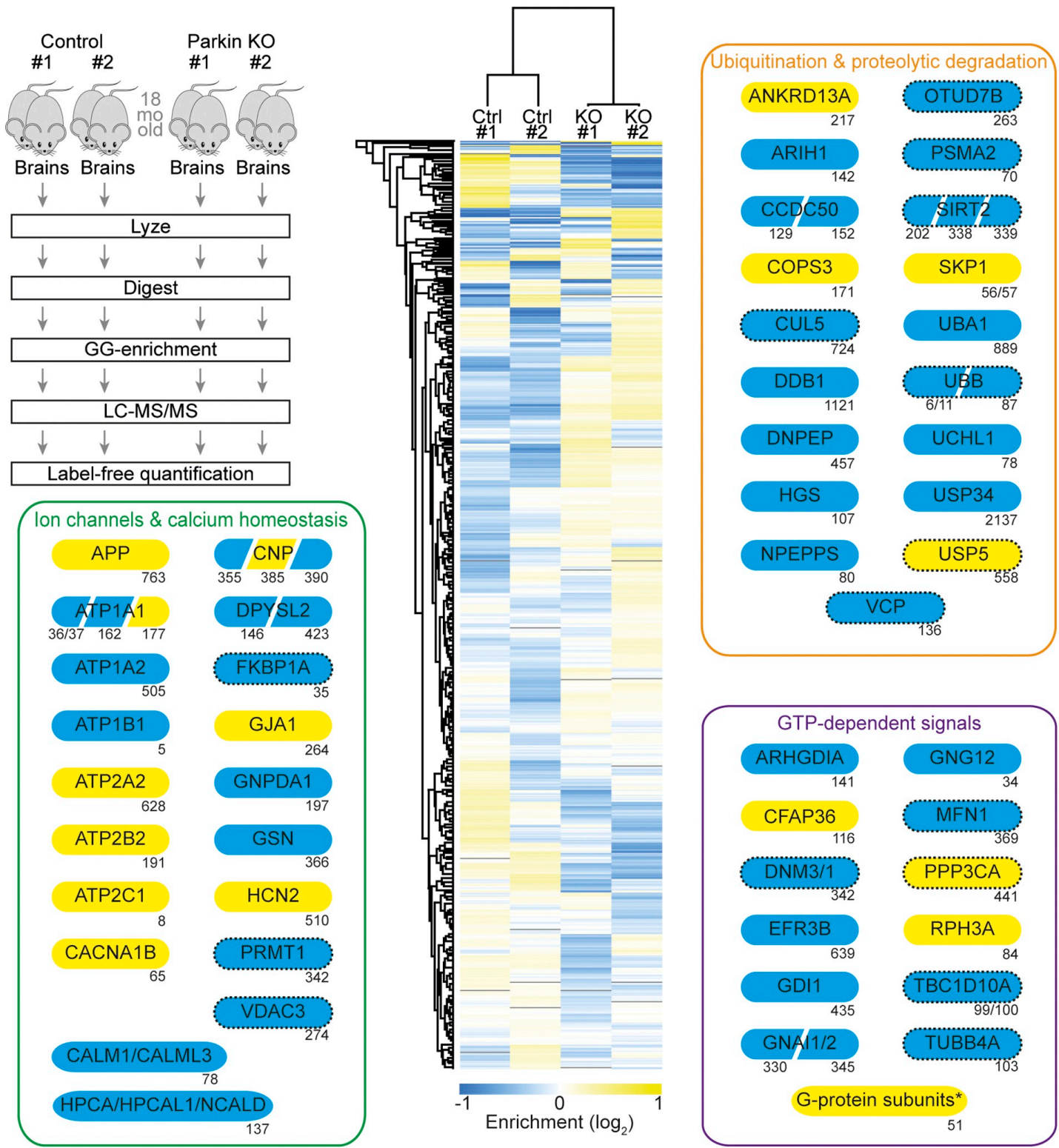

Fig. 1. Scheme of ubiquitylome profiling in old Parkin-KO brains. Study design used four male 18-month-old Parkin-KO mouse brains versus four matched controls, with pooling of brain pairs to achieve sufficient detection depth in mass spectrometry. The heat map of ubiquitin-remnant K-GG motifs for each factor with $>2$-fold dysregulation illustrates sample variability and enrichment. For three significantly enriched pathways, individual factors with downregulation (blue colored symbols) or upregulation (yellow) are shown with the dysregulated ubiquitylation site (residue number). A dotted frame around the protein symbol indicates previously identified Parkin substrate candidates. Oblique white lines within the protein symbol separate ubiquitylated peptides with differential regulation. The asterisk refers to Table 1 for the identity of various G-protein subunits. (For interpretation of the references to colour in this figure legend, the reader is referred to the web version of this article.)

\section{Introduction}

Parkinson's disease (PD) is being intensely investigated as the second most frequent neurodegenerative disease associated with old age. Particularly a monogenic juvenile variant, PARK2, is prominent since it accounts for up to $50 \%$ of autosomal recessive familial PD cases with early onset ( $<40-50$ years, often before 20 years). It is characterized by relatively slow progression and a good long-term response to levodopa therapy, but frequently leads to levodopa-induced dyskinesias (Corti et al., 2011). PARK2 is caused by mutations in an E3 ubiquitin protein ligase enzyme, named Parkin, which relocalizes from the cytosol to the surface of mitochondria during cellular stress periods, thus controlling their repair, fusion-fission balance and mitophagy (Gehrke et al., 2015; Vives-Bauza et al., 2010; Ziviani et al., 2010). In view of the importance of mitochondrial dysfunction for PD pathogenesis (Lin and Beal, 2006), it is crucial to identify the ubiquitylation substrates of Parkin. Assays in vitro and co-immunoprecipitation usually in peripheral cells identified about 40 Parkin target candidates (Imai and Takahashi, 2004; Savitt et al., 2006; Seirafi et al., 2015), but in Parkin-deletion mouse brain and PARK2 patient brain the expected 
accumulation of most factors could not be confirmed (Periquet et al., 2005).

The recent advent of high-throughput sequencing proteome profiling made further advances possible. In a pioneering 2D-electrophoresis and mass-spectrometry study, the ventral midbrain tissue of Parkindeficient mice was documented to display multiple dysregulations of mitochondrial proteins and respiratory complex functions (Palacino et al., 2004). The administration of mitochondrial uncoupling agents such as CCCP or FCCP was shown to synchronize Parkin relocalization to mitochondria, permitting the identification of direct Parkin substrates among mitochondrial outer membrane proteins, such as Porin (VDAC1-3), Mitochondrial Precursor Protein Import Receptor (TOMM70A), the GTPases Mitofusin (MFN1-2), Mitochondrial Fission 1 Protein (FIS1), Dynamin-related Protein 1 (DRP1 or DNM1L), MitoNEET (CISD1-2), Hexokinase (HK1-2), mitochondrial Rho GTPase (MIRO) and Bcl2-Associated X Protein (BAX) (Bertolin et al., 2013; Birsa et al., 2014; Gegg et al., 2010; Geisler et al., 2010; Johnson et al., 2012; Okatsu et al., 2012; Rojansky et al., 2016; Wang et al., 2011).

These substrates were later confirmed when modern approaches of quantifying ubiquitylated peptides in MS were applied to peripheral tumor cells such as HCT116 or HeLa with overexpression of Parkin (Ordureau et al., 2015a,b; Sarraf et al., 2013). Further analyses of the ubiquitylome in Parkin-mutant HeLa cells and embryonic stem-cell derived neurons have now defined the sequential steps involved in ubiquitylation dynamics at the mitochondrial outer membrane during stress periods, at least for abundant and ubiquitous Parkin substrates like VDAC1-3, CISD1-2, HK1 and MFN2 (Ordureau et al., 2018).

However, analyses of aged brain tissue by hypothesis-free global ubiquitylomics are only starting, with a pioneer project in Drosophila melanogaster recently confirming VDACs, CISD2, TOMM70, while identifying two dozen putative Parkin substrates that are novel and involved in vesicle dynamics rather than mitochondria (Martinez et al., 2017, 2018). A role of Parkin and its activator PINK1 for the Rab cycle of cytosolic vesicle was also substantiated by independent studies (Hammerling et al., 2017a,b; Lai et al., 2015; Song et al., 2016; Yamano et al., 2018).

Beyond these molecular details, the overall functional consequences of Parkin/PINK1 loss-of-function for the brain and motor behavior are well studied. The relevant mouse mutants show reduced spontaneous movement activity with dopaminergic deficits at advanced age (Dehorter et al., 2012; Gispert et al., 2009; Itier et al., 2003), weaker synaptic strength, paired-pulse alterations and altered long-term potentiation (Hanson et al., 2010; Kitada et al., 2007), enhanced sensitivity to group II mGlu receptor activation at corticostriatal synapses (Martella et al., 2009), altered $\mathrm{Ca}^{2+}$ homeostasis dependent excitability (Bishop et al., 2010; Carron et al., 2014; Gautier et al., 2016) and neuroinflammation (Mouton-Liger et al., 2018; Sliter et al., 2018; Torres-Odio et al., 2017), all of which have been explained by the mitochondrial dysfunction.

We have now undertaken a pioneer ubiquitylome survey in brain from aged Parkin-knockout (KO) and appropriately matched wildtype (WT) mice, employing an antibody against the di-glycine (K-e-GG) remnant, which is left on ubiquitylated lysine residues after trypsin digestion, and then immuno-precipitating such peptides from the global brain proteome, in order to identify them with tandem mass spectrometry (IP-MS/MS) in a label-free quantitative manner (Fig. 1). Prominent findings were assessed in independent tissues by additional molecular techniques at the protein and mRNA level, employing the acronym of each factor in uppercase to represent the protein level in the text, while lowercase was used to represent the nucleotide level, according to NIH guidelines. Importantly, the characteristic functional consequences of such dysregulation were demonstrated by electrophysiology in acute brain slices from adult versus aged mice. Overall, the findings concur with previous reports that VDAC3 is a main target of Parkin, but also identify novel neuron-specific changes and physiological consequences that become significant only at old age.

\section{Materials and methods}

\subsection{Mouse breeding and brain dissection}

Animals were housed in individually ventilated cages with fixed light cycle under routine health monitoring at the FELASA-certified Central Animal Facility (ZFE) of the Goethe University Medical School, Frankfurt am Main. They were fed ad libitum. For dissection, mice were sacrificed by cervical dislocation. Subsequently, whole brain hemispheres were removed and frozen immediately in liquid nitrogen. Tissue was stored at $-80^{\circ} \mathrm{C}$ until further use. All procedures were in accordance with the German Animal Welfare Act, the Council Directive of 24 November 1986 (86/609/EWG) with Annex II, and the ETS123 (European Convention for the Protection of Vertebrate Animals). Parkin-knockout (KO) and wildtype (WT) control mice, which were derived from common ancestors and share the strain C57BL/6 genetic background, were bred among homozygotes, with genotyping as previously reported (Fournier et al., 2009; Itier et al., 2003). In all brain tissues under analysis, the genotype was controlled again by quantitative RT-PCR of the Parkin transcript level.

\subsection{Global ubiquitylome}

Brain hemispheres from mice at age of 18 months (four KO versus four WT matched for male sex) were dissected in parallel and shipped on dry ice for the commercial UbiScan ${ }^{\circledast}$ procedure (Cell Signaling Technology) (Kim et al., 2011; Udeshi et al., 2013). To have sufficient brain tissue for ubiquitylome profiling, always two brains of $650 \mathrm{mg}$ wet weight were pooled, resulting in 4 LC-MS/MS experiments (experimental design illustrated in Fig. 1). In short, tissue extracts were protease-digested and subjected to $\mathrm{C} 18$ solid-phase extraction. The lyophilized peptides were immunoprecipitated by protein-A/G-agaroseimmobilized ubiquitin-remnant motif antibodies \#3925. Peptides were loaded directly onto a $10 \mathrm{~cm} \times 75 \mu \mathrm{m}$ PicoFrit capillary column packed with Magic C18 AQ reversed-phase resin. The column was developed with a 90 min linear gradient of acetonitrile in $0.125 \%$ formic acid delivered at $280 \mathrm{nl} / \mathrm{min}$. The MS parameter settings were as follows: MS Run Time $96 \mathrm{~min}, \mathrm{MS} 1$ Scan Range (300.0-1500.00), and Top 20 MS/ MS (Min Signal 500, Isolation Width 2.0, Normalized Coll. Energy 35.0, Activation-Q 0.250, Activation Time 20.0, Lock Mass 371.101237, Charge State Rejection Enabled, Charge State 1+ Rejected, Dynamic Exclusion Enabled, Repeat Count 1, Repeat Duration 35.0, Exclusion List Size 500, Exclusion Duration 40.0, Exclusion Mass Width Relative to Mass, Exclusion Mass Width $10 \mathrm{ppm}$ ). MS/MS spectra were evaluated using SEQUEST 3G and the Sorcerer 2 platform from Sage-N Research (v4.0, Milpitas, CA, USA) [36]. Searches were performed against the most recent update of the NCBI Mus musculus database with mass accuracy of $\pm 50 \mathrm{ppm}$ for precursor ions and $1 \mathrm{Da}$ for product ions. Results were filtered with mass accuracy of $\pm 5 \mathrm{ppm}$ on precursor ions and presence of the intended motif (Me-R). Peptide identification with relative quantification by mass spectrometry occurred by LC-MS/MS analysis using LTQ-Orbitrap-VELOS with ESI-CID Sorcerer search.

Bioinformatic processing used the maximum \% coefficient of variation (\% CV) to control replicate reproducibility. Using a 5\% default false positive rate to filter the Sorcerer results, this procedure yielded a total of 6746 redundant ubiquitinated peptide assignments to 3427 nonredundant ubiquitinated peptides. The quantitative data from the four KO and four WT mice were pooled to derive the respective fold change. The original data are available from the authors upon request.

\subsection{Bioinformatic analyses}

For protein-protein interaction (PPI) network analysis, the software tool String v.10 (https://string-db.org/) with standard settings was employed to visualize networks of relevant dysregulations as previously described (Torres-Odio et al., 2017). As recommended, gene symbols of 
factors with $>2$-fold dysregulation were entered into the Multiple Proteins window with the Mus musculus option, the matching of the input with the correct factors was accepted, and the graphic interaction diagram was generated and archived. The Analysis button generated automated network statistics; significant functional enrichments of GO (Gene Ontology) terms and KEGG pathways were exported to EXCEL files.

\subsection{Quantitative immunoblotting}

Total proteins from 4 WT versus 4 Parkin-KO mouse brain hemispheres were isolated as described, with modifications (Lastres-Becker et al., 2016). Frozen tissues were homogenized on ice with a Dounce grinder and homogenates were lysed in RIPA buffer with $50 \mathrm{mM}$ Tris$\mathrm{HCl}$ (pH 8.0), $150 \mathrm{mM} \mathrm{NaCl}, 1 \% \mathrm{NP}-40,0.5 \%$ Na-deoxycholate, $0.1 \%$ SDS and protease inhibitor cocktail (Roche), respectively. Total lysates were briefly sonicated on ice, and cell debris was removed by centrifugation. Samples of $20 \mu \mathrm{g}$ were heated at $90^{\circ} \mathrm{C}$ for $5 \mathrm{~min}$ and then separated in tris-glycine polyacrylamide gels, using Precision Plus Protein $^{\mathrm{TM}}$ All Blue Standards as size marker. Transfer to nitrocellulose membranes (Protran, GE Healthcare) was done at $50 \mathrm{~V}$ for $90 \mathrm{~min}$, with blocking in $5 \%$ BSA solution in $1 \times$ TBS-T for $1 \mathrm{~h}$ at room temperature (RT). Incubation with primary antibodies against HPCA (1:1000, ab24560, Abcam), ATP1A2 (1:1000, MA3-928, Thermo Scientific), GNA11 (1:1000, GTX54123, GeneTex) and $\beta$-Actin (1:5000, A5441, Sigma-Aldrich) occurred in $1 \times$ TBS-T solutions overnight at $4{ }^{\circ} \mathrm{C}$. Fluorescent-labeled $\alpha$-mouse (Li-Cor IRDye 800CW, 1:15.000) and $\alpha$ rabbit (Li-Cor IRDye 680RD, 1:15.000) were secondary antibodies, with fluorescence detection by Li-Cor Odyssey Classic Instrument.

\subsection{Quantitative reverse-transcriptase polymerase chain reaction}

Total RNA of 4 WT and 4 Parkin-KO mouse brain hemispheres was extracted with TRI reagent (Sigma) and cDNA was made with the Superscript IV Kit (Invitrogen), both following the manufacturer's instructions. Quantitative reverse-transcriptase (RT) polymerase chain reaction (qPCR) was performed with TaqMan Gene Expression Assays (Applied Biosystems) in cDNA from $20 \mathrm{ng}$ total RNA in $20 \mu \mathrm{l}$ reactions with $2 \times$ master mix from Roche in a StepOnePlus Real-Time PCR system (Applied Biosystems). All assays were normalized against Tbp expression levels. The analysis of the data was carried out with the $2^{-\Delta \Delta C T}$ method (Livak and Schmittgen, 2001). The following TaqMan Assays were employed: Atp1a1-Mm00523255_m1, Atp1a2- Mm00617899_m1, Atp1a3Mm00523430_m1, Atp1b1- Mm00437612_m1, Atp2a1-Mm01275320 m1, Atp2a2- Mm01201431_m1, Atp2b2- Mm00437640_m1, Atp2b3Mm01191090_m1, Atp2c1- Mm00723484_m1, Calm1- Mm00486655. m1, Gdi1- Mm00802649_m1, Gna11- Mm01172792_m1, Gnai1Mm01165301_m1, Gnai2- Mm00492379_g1, Gnai3- Mm00802670_m1, Gnao1- Mm00494677_m1, Gnaz- Mm01150269_m1, Gng12- Mm011838112_m1, Hpca- Mm00650703_m1, Park2- Mm00450186_m1 and TbpMm00446973_m1.

\subsection{Dissection of vital brainstem slices}

The slice dissection, electrophysiological analyses and neurobiotin immunostaining of LC neurons were performed as previously described (Matschke et al., 2018). Briefly, mice were anesthetized with isoflurane gas/intraperitoneal ketamine. They were perfused transcardially with an ice-cold glucose/magnesium-rich preparation [in mM: $2.5 \mathrm{KCl}, 1.25$ $\mathrm{NaH}_{2} \mathrm{PO}_{4}, 10 \mathrm{MgSO}_{4}, 20$ PIPES, 10 Glucose, 200 Saccharose, $0.5 \mathrm{CaCl}_{2}$, $\mathrm{pH}$ 7.35] to ensure tissue vitality and brains were removed rapidly.

Coronal sections $(180-200 \mu \mathrm{m}$ thick) were made using a Campden MA752 vibratome (Campden Instruments) within the ice-cold preparation. After sectioning, slices were maintained at $30^{\circ} \mathrm{C}$ for $30 \mathrm{~min}$ and afterwards at RT for $30 \mathrm{~min}$, submerged in artificial cerebrospinal fluid (ACSF) composed of (in mM): $125 \mathrm{NaCl}, 2.5 \mathrm{KCl}, 25 \mathrm{NaHCO}_{3}, 1.25$
$\mathrm{NaH}_{2} \mathrm{PO}_{4}, 2 \mathrm{CaCl}_{2}, 1 \mathrm{MgCl}_{2}$ and 25 glucose, equilibrated to $\mathrm{pH} 7.4$ with $95 \% \mathrm{O}_{2} / 5 \% \mathrm{CO}_{2}$. Subsequently, slices were kept at RT for up to $5 \mathrm{~h}$. For electrophysiological recordings slices were transferred to a recording chamber mounted on an upright microscope and continuously perfused with ACSF, unless specified otherwise. LC neurons were visualized with a Zeiss AxioCam MRm camera (Carl Zeiss Microscopy, LLC, USA) mounted on a Zeiss Examiner.D1 microscope (Carl Zeiss Microscopy, LLC, USA), equipped with a $40 \times / 0.75$ phase contrast, water immersion objective, standard EGFP filter and Texas red filter sets. Brainstem slices containing a clearly defined LC at the edge of the fourth ventricle were used for the experiments.

\subsection{Slice patch clamp recordings}

Patch clamp recordings were performed at RT in LC neurons identified by their large somata and pacemaker frequencies between 1 and $5 \mathrm{~Hz}$. Patch pipettes were prepared from borosilicate glass capillaries GB 150TF-8P (Science Products, Hofheim, Germany), and had tip resistances between 3 and $5 \mathrm{M} \Omega$. Conventional tight seal whole-cell voltage clamp experiments were conducted with ACSF as external solution and an internal solution containing (in $\mathrm{mM}$ ): $135 \mathrm{~K}$-Glutamate, $5 \mathrm{KCl}$, 10 HEPES, 0.1 EGTA, $2 \mathrm{MgCl}_{2}, 2 \mathrm{MgATP}, 0.2 \mathrm{Li}_{2} \mathrm{GTP}$ and adjusted to $\mathrm{pH} 7.4$ with $\mathrm{KOH}$. All patch clamp recordings were made using a Multiclamp 700B amplifier (Molecular Devices, Sunnyvale CA, USA) and Clampex 10.0 software (Molecular Devices, Sunnyvale CA, USA). Data were digitized at $10 \mathrm{kHz}$ with a Digidata $1440 \mathrm{~A}$ digitizer (Molecular Devices, Sunnyvale CA, USA) and filtered at $1-5 \mathrm{kHz}$. Electrode capacitance was compensated and only recordings with a constant series resistant $<30 \mathrm{M} \Omega$ were used for analysis. All experiments were done in several mutant animals and controls $(N=3-6)$.

\subsection{Immunostaining}

For neurobiotin (NB) stainings, LC neurons were filled by adding $0.1 \%(w / v) \mathrm{NB}$ to the pipette solution. After finishing patch clamp recordings, slices were subsequently fixed in a solution composed of $4 \%$ PFA in 0.1 M phosphate buffered saline (PBS), pH 7.4. After fixation for $48 \mathrm{~h}$, slices were stored in $30 \%$ sucrose in $0.1 \mathrm{M}$ PBS at $4{ }^{\circ} \mathrm{C}$. For the subsequent immunohistochemical staining, sections were blocked in $10 \%$ horse serum followed by overnight incubation with primary antityrosine-hydroxylase antibody (Calbiochem, \#657012, working dilution 1:1000). Anti-Rabbit Alexa 568 (Invitrogen, \#A11011, working dilution 1:750) served as secondary antibody and co-staining was performed with streptavidin conjugated with Alexa 488 (Invitrogen, \#S11223, working dilution 1:1000).

Images were acquired with an Axio Observer.Z1 microscope (Carl Zeiss Microscopy, LLC, United States) equipped with standard EGFP filter and Texas red filter sets and an Axio Cam MRm camera (Carl Zeiss Microscopy, LLC, United States). Digital images were processed using ZEN 2.3 (Carl Zeiss Microscopy, LLC, United States).

\subsection{Statistical analyses}

Data are reported as means \pm S.E.M. For expression studies, statistical significance was assessed using unpaired Student's $t$-test in the GraphPad Prism 7 software. For electrophysiological studies, data were analyzed using Microsoft Excel and OriginPro (OriginLab Corp., Guangzhou, China). Data sets were tested for Normality using the Shapiro Wilk test and for equal variances using the Levene's test. In normally distributed data sets, statistical significance was calculated using paired or unpaired Student's $t$-test. Otherwise non-parametric statistical tests, e.g. the Wilcoxon signed-rank test for paired data or the Mann-Whitney- $U$ test for unpaired data were used. * stands for $P<.05 ; * *$ for $P<.01 ; * * *$ for $P<.001$. 
Table 1

Global ubiquitylome profile of brain from 18-month-old Parkin-KO mice. The table illustrates factors (yellow background for known Parkin substrates) and their relevant ubiquitylation sites (highlighted by asterisk) with $>2$-fold dysregulation (red background for increases, blue background for decreases) and literature references. Potential novel Parkin substrates that are not neuron-specific were highlighted with light yellow background. Potential novel Parkin substrates that are neuron-specific and showed $>$ 4-fold downregulation were highlighted with purple background. Putative compensatory upregulations of G-protein subunits were highlighted with green background.

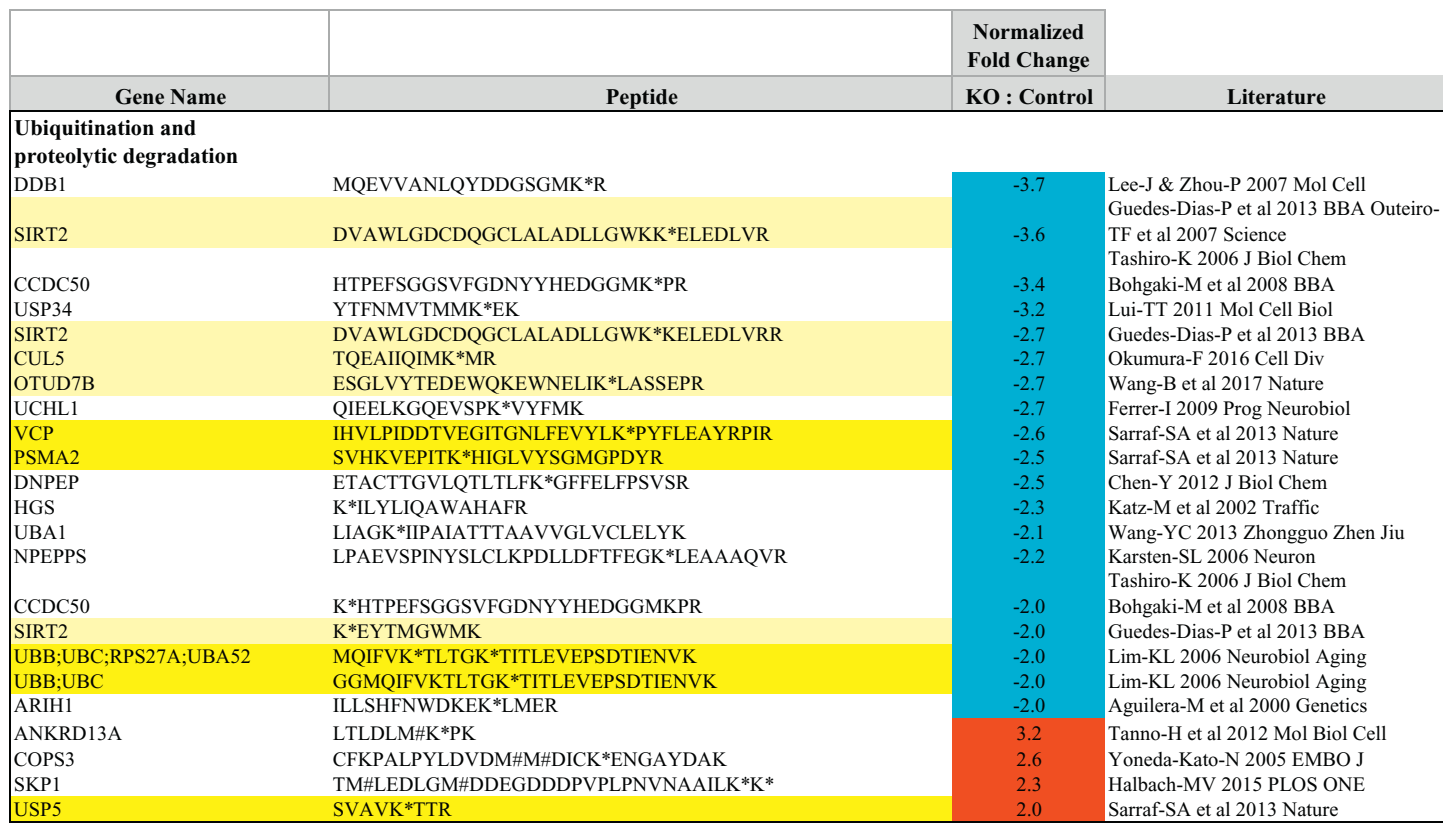

\section{Ion channels \&}

calcium homeostasis

(2)

VDAC3

VDAC3
HPCA;HPCAL1;NCALD

CALM1;CALML3

ATP1A1;ATP1A3;ATP1A4

DPYSL2

DPYSL2

FKBP1A

FKBP1A

ATP1A2

ATP1A2

GSN

CNP

CNP

ATP1B1

ATP1A1

GNPDA1

ATP2C1

APP

CNP

GJA1

ATP1A1

ATP2B2

CACNA1B

ATP2A2

$\mathrm{HCN} 2$

NFNAGGHK*VGLGFELEA MVSSVMK*MPEDESTPEK

KMK*DTDSEEEIREAFR

IMESFK*NMVPQQALVIR

TISAK*THNSALEYNIFEGMECR

SCCDYSLHVDITEWHK*GIQEEMEALVK

RGQTCVVHYTGMLEDGK*K

EDSPQSHVLVMK*GAPER

TGEEIFGTIGMRPNAK*NNR

TASDFISK*MQYPR

KMEVK*AIFTGYYGK

AHVTLGCAADVQPVQTGLDLLDILQQVK*GGSQGEAVGELPR

GK*AKEEGSWKK

DMDELK*K*EVSMDDHK

VPTMALTVGVGTVM\#DAK*EVM\#ILITGAHK

FQK*IPNVENETM\#IPVLTSK

M\#QQNGYENPTYK*FFEQM\#QN

WM\#LSLTK*K

DCGSPK*YAYFNGCSSPTAPLSPM\#SPPGYK

NGEK*M\#SINAEDVVVGDLVEVK

IEQEQK*FTVVR

TM\#ALYNPIPVK*QNCFTVNR

VIM\#ITGDNK*GTAVAICR

K*LVASM\#PLFANADPNFVTAM\#LTK

\section{GTP-dependent signals}

TBC1D10A

MFN1

GNAI1;GNAI2

GNAI1;GNAI2

EFR3B

TUBB4A

DNM3;DNM1

GNG12

ARHGDIA

WLDMLNNWDKWMAK*K*

NQMNLLTLDVKK*

MAGSAFDFENMK*R
NVQFVFDAVTDVIIK*NNLKDCGLF

EIYTHFTCATDTK*NVQFVFDAVTDVIIKNNLK

KKEAPYMLPEDVFVEK*PR

SGPFGQIFRPDNFVFGQSGAGNNWAK*GHYTEGAELVDAVLDVVR

ALLQMVQQFAVDFEK*R

IKVSK*ASADLMSYCEEHAR

IDK*TDYMVGSYGPR

G-alpha1(i);G-alpha2(i);G-alpha3(i); GNAO1;GNAT2;GNAT1;GNAT3;

G-alpha(z)

CFAP36

PPP3CA
STIVK $* \mathrm{QM} \# \mathrm{~K}$

AM\#M\#VQK*NIEM\#QLQAIR

GLTPTGM\#LPSGVLSGGK*QTLQSAIK

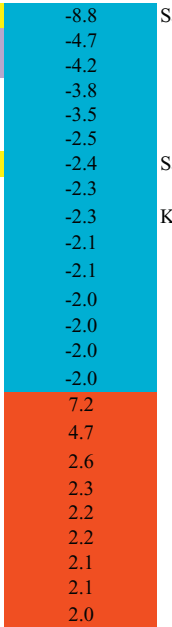

Sarraf-SA et al 2013 Nature

Sarraf-SA et al 2013 Nature

Kim-HJ et al 2016 Elife

im-HJ et al 2016 Elife 
Table 1 (continued)

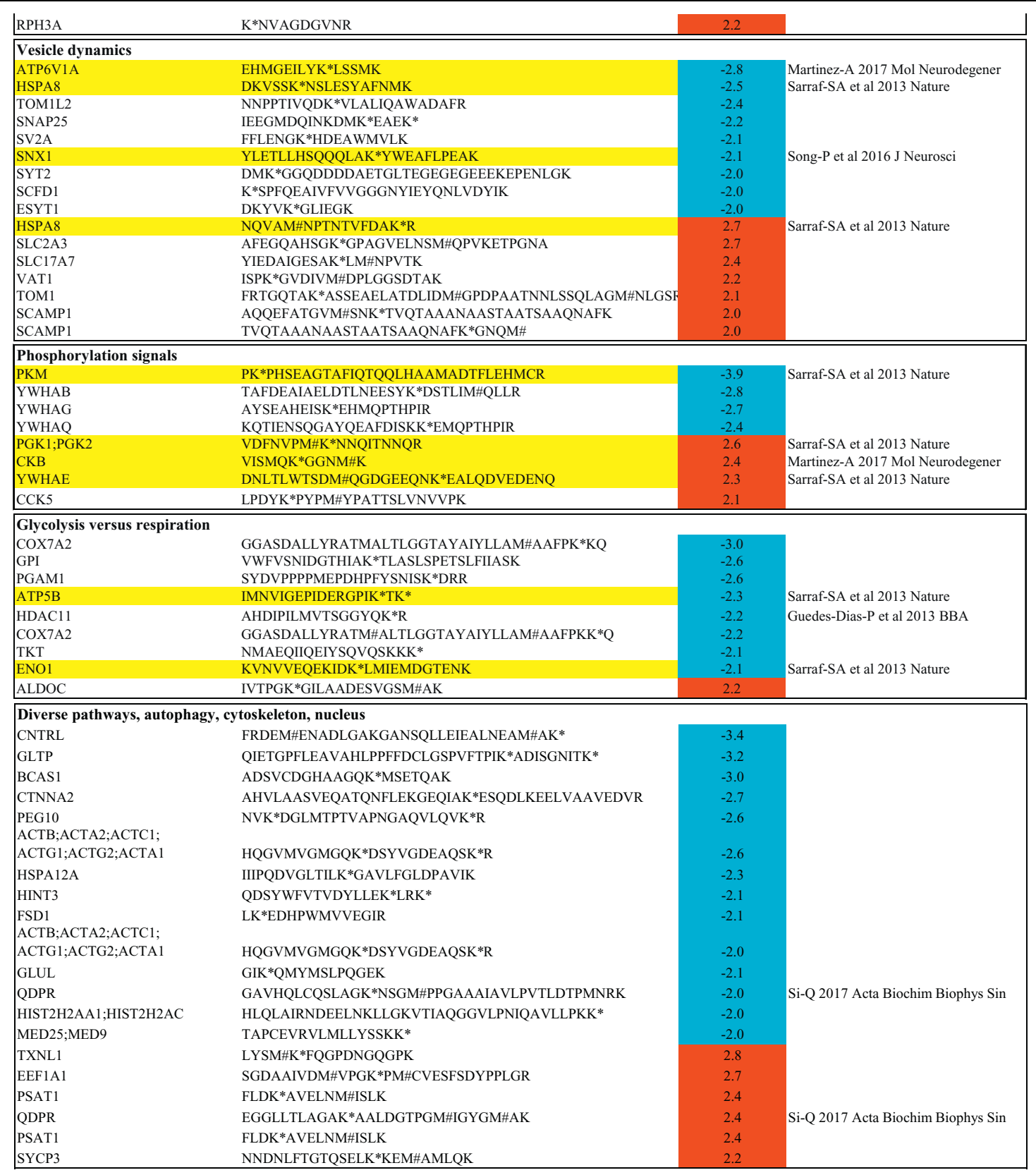




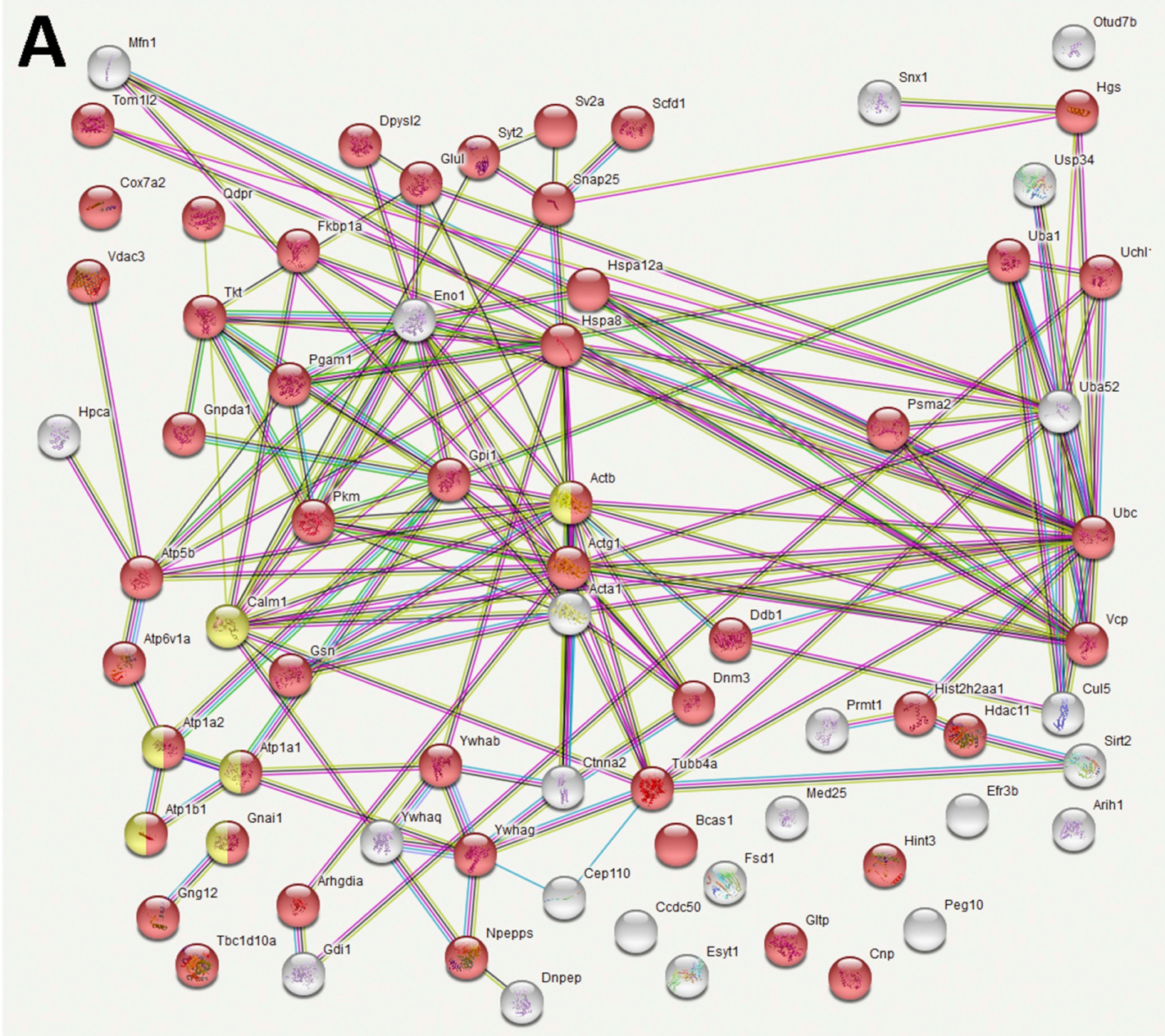

B

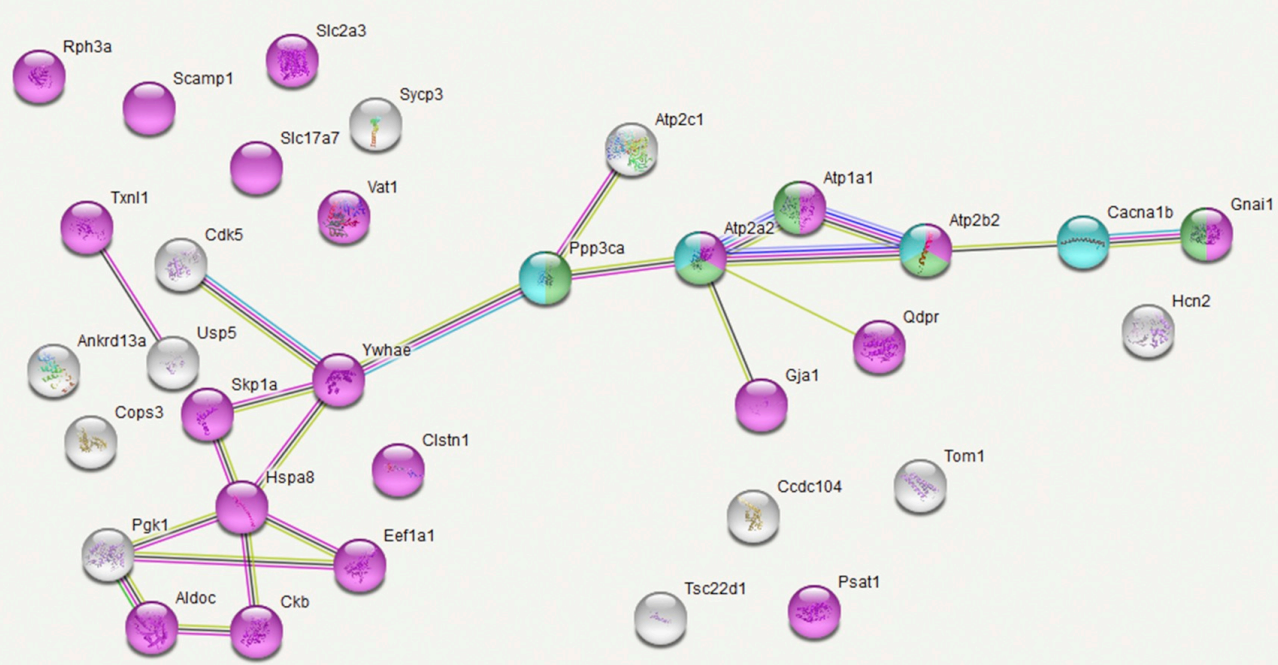

(caption on next page) 
Fig. 2. Diagram of protein-protein interactions among the factors with ubiquitylation changes beyond two-fold, according to the STRING website. (A) All downregulations are shown, with factors involved in ubiquitin and protein turnover placed on the right upper margin, those involved in mitochondrial function placed into the left upper margin, Hippocalcin at the left middle margin. Red symbols indicate vesicle-associated factors, while yellow symbols illustrate factors that are relevant for stimulus-dependent acid secretion, with Calmodulin among them. (B) All upregulations are shown, with factors involved in secretory vesicles placed into the left upper corner. Purple symbols indicate proteins at membrane-bounded vesicles, e.g. the Rab3a-interactor Rph3a in the upper left corner. Light blue stands for the calcium signaling pathway, including several calcium channels. Light green illustrates the cGMP PKG signaling pathway components, e.g. Gnai1 placed at the right margin. (For interpretation of the references to colour in this figure legend, the reader is referred to the web version of this article.)

\section{Results}

\subsection{Aged Parkin-KO brain ubiquitylome profile overview}

\subsubsection{Downregulations were prominent}

The brain ubiquitylome of 18-month-old Parkin-KO mice showed 114 peptide dysregulations that had $>2$-fold effect size (Table 1 ), including 21 known Parkin substrates (highlighted by yellow background in Table 1). Downregulation of 79 ubiquitylation sites was documented (highlighted by blue background in Table 1), providing a promising basis to identify novel neuron-specific direct Parkin ubiquitylation targets among additional indirect effects. Interestingly, 34 upregulations were also observed (highlighted by red background in Table 1), which may represent random consequences of the perturbed protein degradation machinery as well as compensatory efforts.

\subsubsection{Some factors harbored both down- and upregulations}

Downregulations and possibly homeostatic upregulations coincided in selected factors (shown as symbols with blue and yellow colour in Fig. 1), in particular a moderate ubiquitylation deficit of a peptide from GNAI1/GNAI2 ( -2.2 -fold at amino acid $\$ 345 / 346$ and at $\S 330 / 331)$ was observed together with a strongly increased ubiquitylation $(+5.4-$ fold at $\$ 47 / 51$ ) of a peptide from the G-protein subunits GNAI1/ GNAI2/GNAI3/GNAO1/GNAT1/GNAT2/GNAT3/GNAZ, highlighted with green background in Table 1 . Similarly, an ubiquitylation deficit in a peptide from ATP-driven Na/K-pumps ATP1A1/ATP1A3/ATP1A4 $(-3.8$-fold at $\$ 152 / 162 / 172)$ and in a peptide purely from ATP1A1 $(-2.0$-fold at $\$ 36 / 37)$ contrasted with an excessive ubiquitylation of ATP1A1 (+2.2-fold at §177). Furthermore, an ubiquitylation deficit in the immunity modifier (Girirajan et al., 2008) and ubiquitin/clathrin/ Tollip interactor TOM1L2 ( -2.4 -fold at §116) occurred together with excessive ubiquitylation of its homolog TOM1 $(+2.1$-fold at §311). In addition, an ubiquitylation deficit in the autophagy modulator QDPR at $\S 164$ ( -2.0 -fold) accompanied an excessive ubiquitylation of QDPR at $\S 135$ ( + 2.4-fold). Finally, an ubiquitylation deficit in CNP $(-2.0$-fold at $\S 335 / 355 ;-2.1$-fold at $\S 370 / 390$ ) was observed in parallel with excessive ubiquitylation in CNP (+2.6-fold at \$365/385) and might influence its association with Calmodulin and its mitochondrial regulation in response to calcium (Azarashvili et al., 2009; Myllykoski et al., 2012). In these proteins it may be crucial for the cell to maintain the steady-state levels normal, so that indirect compensatory efforts counterweigh the pathological ubiquitylation deficits triggered by Parkin deficiency.

\subsubsection{The 3 biggest downregulations occurred in calcium regulators}

Focusing primarily on the strongest downregulations (beyond -4.0 fold cutoff), an exceptional -8.8-fold ubiquitylation deficit was observed for VDAC3 as member of the Porin family and as known Parkin substrate, reproducing previous reports and confirming the validity of our approach. This protein family controls mitochondrial calcium homeostasis at the mitochondrial outer membrane, which is crucial when neuronal pacemaker activity changes due to stimulation, with subsequent increases of energy demand (Cali et al., 2014; Surmeier et al., 2012). A peptide from the neuron-specific calcium sensors Hippocalcin, Hippocalcin-Like 1 or Neurocalcin Delta (HPCA, HPCAL1 or NCALD) showed the second strongest effect ( -4.7 -fold), and a peptide from the calcium-buffering factors Calmodulin or Calmodulin-Like 3
(CALM1 or CALML3) showed a third substantial effect ( -4.2 -fold) Given that mitochondrial dysfunction in Parkin-deficient brain may alter the mitochondrial calcium storage and calcium-dependent excitability of neurons, it is possible that Hippocalcin and Calmodulin change due to indirect cellular adaptations rather than as direct Parkin substrates, but these findings had high credibility and were investigated further by quantitative RT-PCR, quantitative immunoblots and electrophysiology.

\subsection{The KO brain ubiquitylome contains $>2$-fold downregulation of 15 known PARKIN substrates}

\subsubsection{Several established Parkin ubiquitylation targets were confirmed}

It was important to note that the ubiquitylation of overall cellular pools of ubiquitin (UBB/UBC) as a known Parkin substrate changed -2.0-fold, an observation with consequences for the turnover of practically all proteins. Therefore, in subsequent bioinformatics analyses only effects that surpassed this threshold were taken into account. Apart from VDAC3 (-8.8-fold) and UBB/UBC $(-2.0)$, the downregulation of other known Parkin substrates ranged from -3 to -2 fold levels (overview in Fig. 1). This included the calcium release modulator FKBP1A (-2.4); several GTPases, such as the mitochondrial fusion factor MFN1 $(-2.8)$, the microtubule and LRRK2 interactor TUBB4A $(-2.2)$ and the vesicle endocytosis factors DNM1/DNM3 $(-2.1)$; the vesicle ATPase ATP6V1A $(-2.8)$, the Golgi vesicle ATPase VCP $(-2.6)$ and the vesicular trafficking modulator SNX1 $(-2.1)$; the molecular chaperone HSPA8 $(-2.5$ at $\S 539$, but +2.7 at $\S 71)$ and the proteasome subunit PSMA2 $(-2.5)$; the glycolytic kinase PKM $(-3.9)$, the glycolytic enzyme ENO1 $(-2.1)$ and the respiratory ATP synthase subunit ATP5B $(-2.3)$ (relevant literature references in Table 1).

\subsubsection{Four ubiquitylation downregulations may represent novel Parkin targets}

Four factors hold immediate promise as novel potential Parkin substrates in view of their involvement in PD pathogenesis, although they are not neural-specific: First, a strongly decreased ubiquitylation ( -3.6 -fold at $\$ 339 / 302 / 269,-2.7$ at $\$ 338 / 301 / 268$ and -2.0 -fold at $\S 202 / 202 / 165 / 132)$ was observed for SIRT2, which is a target of Cb1dependent ubiquitylation during EGFR-endocytosis, deacetylates the p65 subunit of NF-kB and protects against Parkinsonian alpha-synuclein neurotoxicity (Outeiro et al., 2007). It also deacetylates the transcriptional factor FOXO3 to stimulate its SCF(SKP2)-mediated ubiquitination, in a pathway that modulates dopaminergic differentiation as well as nigrostriatal vulnerability to the neurotoxin MPTP (Liu et al., 2012; Pino et al., 2014; Szego et al., 2017), via AKT/GSK-3ß/ $\beta$-catenin. Second, the reduced ubiquitylation of a modulator of bacteria-containing endosomes and autophagy (Minowa-Nozawa et al., 2017), the RAB35-associated, GTPase activating protein TBC1D10A ( -3.7 at $\S 99$ and §100) may hold particular interest, since TBC1D15 as another RabGAP was already documented to act downstream of Parkin (Yamano et al., 2018). Third, the reduced ubiquitylation of the deubiquitinase OTUD7B ( -2.7 -fold at §263) may deserve special attention, given that it acts as inhibitor of NF-kB via the known Parkin-substrate TRAF3 (TNF Receptor Associated Factor 3, a RING-Type E3 Ubiquitin Transferase), as modulator of mTOR-dependent growth together with TRAF2, and as modifier of EGFR turnover (Pareja et al., 2012; Wang et al., 2017; Xin et al., 2018). Fourth, the reduced ubiquitylation of the 
ubiquitin ligase scaffold CUL5 ( -2.7 -fold at $\$ 724$, also named VACM-1 for its vasopressin-dependent action on calcium mobilization and cAMP production) is meaningful in this context, given that CUL5 interacts with TRAF6 to promote lipopolysaccharide signals (Zhu et al., 2016), which are known to exacerbate Parkin-dependent pathology (Tran et al., 2011; Zhong et al., 2016). All four factors are highlighted with light yellow background in Table 1 .

\subsubsection{Neural stimulation pathways are prominently affected}

Thus, specific pathways such as calcium homeostasis, GTP-binding regulators, membrane-associated ATPases, endocytosis, protein turnover modulators and energy metabolism appeared as recurrent functions among the known and potential Parkin substrates (see Fig. 1). For an unbiased investigation of such clustering, we performed a formal bioinformatics enrichment assessment of GO terms, KEGG pathways and Pfam domains among all documented $>2$ fold ubiquitylation changes in Table 1 at the STRING website (all results with ranking are shown in several datasheets of Supplementary Table 1), hoping to gain insight into mitochondrial and extra-mitochondrial effects of Parkin.

\subsection{Evidently reduced ubiquitination of other factors in the ubiquitin/ protein degradation pathways}

3.3.1. Bioinformatic enrichment of protein degradation factors within ubiquitylome profile

Among the $>2$-fold downregulations, enrichments of the GO Biological Process terms "modification-dependent protein catabolic process" (false discovery rate FDR $=0.001$ ), of "ubiquitin-dependent protein catabolic processes" $(\mathrm{FDR}=0.003)$ and of proteolysis $($ FDR $=0.005)$ seemed obviously relevant. This pathway was also enriched among the GO terms Molecular Function. The affected molecules included prominently the ubiquitin E3 ligase subunit DDB1 (-3.7-fold at amino acid §1121), which modulates G-protein coupled receptor turnover and may associate with the COP9 signalosome during responses to abnormal DNA (Cavadini et al., 2016; Li et al., 2018). It is interesting to note that an excessive ubiquitylation of the signalosome component COPS3 $(+2.6$-fold $)$ was also observed.

\subsubsection{Strong affection of EGFR and NF-kB turnover regulators}

An almost as strong reduction of ubiquitylation was documented for the beta-catenin modulator, DNA-damage-response factor and NF-kB repressor USP34 (-3.2-fold) (Lui et al., 2011; Poalas et al., 2013; Sy et al., 2013), to similar extent as the EGFR-endocytosis regulator and $\mathrm{NF}-\mathrm{kB}$ repressor CCDC50 ( -3.4 at $\$ 152$ and -2.0 at §129) that contains several ubiquitin-interactor domains (Bohgaki et al., 2008; Tashiro et al., 2006; Tsukiyama et al., 2012). In this context, it is noteworthy that the EGFR-endocytosis repressor ANKRD13A (+3.2-fold at §217) and the CUL1-associated SCF complex subunit SKP1A (+2.3fold at \$56/57) were excessively ubiquitylated.

\subsubsection{Moderate affection of two PD pathogenesis factors}

Weaker reductions of ubiquitylation were observed for CUL5 ( -2.7 fold) as component of E3 ubiquitin-protein ligase complexes, also named Vasopressin-Activated Calcium-Mobilizing Receptor, shown to act as SOCS6-associated repressor of Src/Cas-dependent membrane dynamics and autophagy (Antonioli et al., 2014; Teckchandani et al., 2014); the same extent was also observed for the ubiquitin hydrolase UCHL1 ( -2.7 -fold), which was also named PARK5 since missense mutations were observed in a family with hereditary Parkinson's disease (Leroy et al., 1998); for the proteasome subunit PSMA2 (-2.5fold), which serves as selective target of the SCF ubiquitin E3 ligase complex containing FBXO7, also named PARK15 because of mutations associated with Parkinson's disease (Vingill et al., 2016); finally similar effects were documented for the peptide turnover enzyme DNPEP $(-2.5$-fold) and NPEPPS $(-2.2$-fold) as a synaptic protease (Huber et al., 1999).

\subsubsection{Weak affection of other growth/stress regulators}

Small effect sizes were found for the hepatocyte growth factorregulated tyrosine kinase substrate and ubiquitin-interactor HGS ( -2.3 -fold) (Katz et al., 2002), for the ubiquitin-activating E1 enzyme UBA1 (-2.1-fold), and the Parkin-homologous cullin-RING ubiquitin ligase complex-associated ARIH1 ( -2.0 -fold) that triggers translation arrest in response to DNA-damage (Aguilera et al., 2000; von Stechow et al., 2015).

In summary, several dysregulated factors within the protein turnover pathways are known modulators of endocytotic internalization and inflammatory responses to abnormal DNA. These findings provide a molecular basis, which may explain a recent report that Parkin controls metazoan innate immune defense against bacteria, via modulation of phagocytic uptake and inflammatory responses (Manzanillo et al., 2013).

3.4. Bioinformatic assessment of pathway enrichments points to prominent vesicle pathology and cGMP/calcium dependent signaling

\subsubsection{Decreased ubiquitylation}

Among the downregulations, the unbiased bioinformatics assessment observed a prominent enrichment for "vesicles" with an outstanding number of 48 factors showing dysregulation within the GO terms for Cellular Components (Supplementary Table 1; illustrated as red bullets in Fig. 2A), prominent enrichments for "gastric acid secretion", "cGMP-PKG signaling pathway", "endocrine and other factorregulated calcium reabsorption" and "cardiac muscle contraction" within the KEGG pathways were mainly based on the same factors (ATP-driven channels).

\subsubsection{Increased ubiquitylation}

Among the upregulations, prominent enrichments were found for "cellular ion homeostasis" and "regulation of membrane potential" among the GO terms Biological Processes, for "anion binding" among the GO terms Molecular Functions, for "membrane-bounded vesicles" among the GO terms Cellular Components (illustrated as purple bullets in Fig. 2B), for "cGMP-PKG signaling pathway" and "calcium signaling" among the KEGG pathways (Supplementary Table 1).

\subsubsection{Enrichment of calcium signaling factors}

Beyond the bioinformatically defined pathway components, the importance of calcium homeostasis was also emphasized by the striking upregulation of the Golgi-associated calcium pump ATP2C1 $(\$ 8+7.2-$ fold) and of the Amyloid Beta A4 precursor APP $(\$ 688 / 728 / 763+4.7-$ fold), which is a known modulator of calcium currents during multiple challenges with nicotine or caffeine via sensitization or overfilling of endoplasmic reticulum stores that are maintained by SERCA (ATP2A) pump and mitochondrial calcium efflux (Chin et al., 2006). Further support came from the downregulations of the calcium-regulated actin assembly promoter GSN ( $\$ 317 / 366-2.1$-fold), the calcium oscillation generator GNPDA1 ( $\$ 197-2.0$-fold), the calcium-dependent synaptic vesicle interactor SYT2 (\$115-2.0-fold), and the calcium-dependent interactor with ER/plasma membranes ESYT1 (\$339-2.0-fold). Most importantly, the calcium signaling pathway had already been highlighted by the strong ubiquitylation deficits of Hippocalcin and Calmodulin, marked with purple background in Table 1. Hippocalcin is a neuron-specific $\mathrm{Ca}^{2+}$-sensor, which modulates the pool of cyclic GMP that is catalyzed by the membrane bound guanylate cyclase transduction system (Krishnan et al., 2009) and which was also found to regulate voltage-dependent calcium channels (Helassa et al., 2017). During axonal signal transduction, Hippocalcin gates the calcium activation of KCNQ-dependent potassium currents that mediate the slow afterhyperpolarization (SAHP), which follow any train of action potentials (Kim et al., 2016b; Kim et al., 2012; Tzingounis et al., 2007). Deficiency of Hippocalcin does not lead to adaptation of expression in other neural calcium-sensors such as VILIP-1 / VILIP-3 / NCS-1 (Kobayashi et al., 

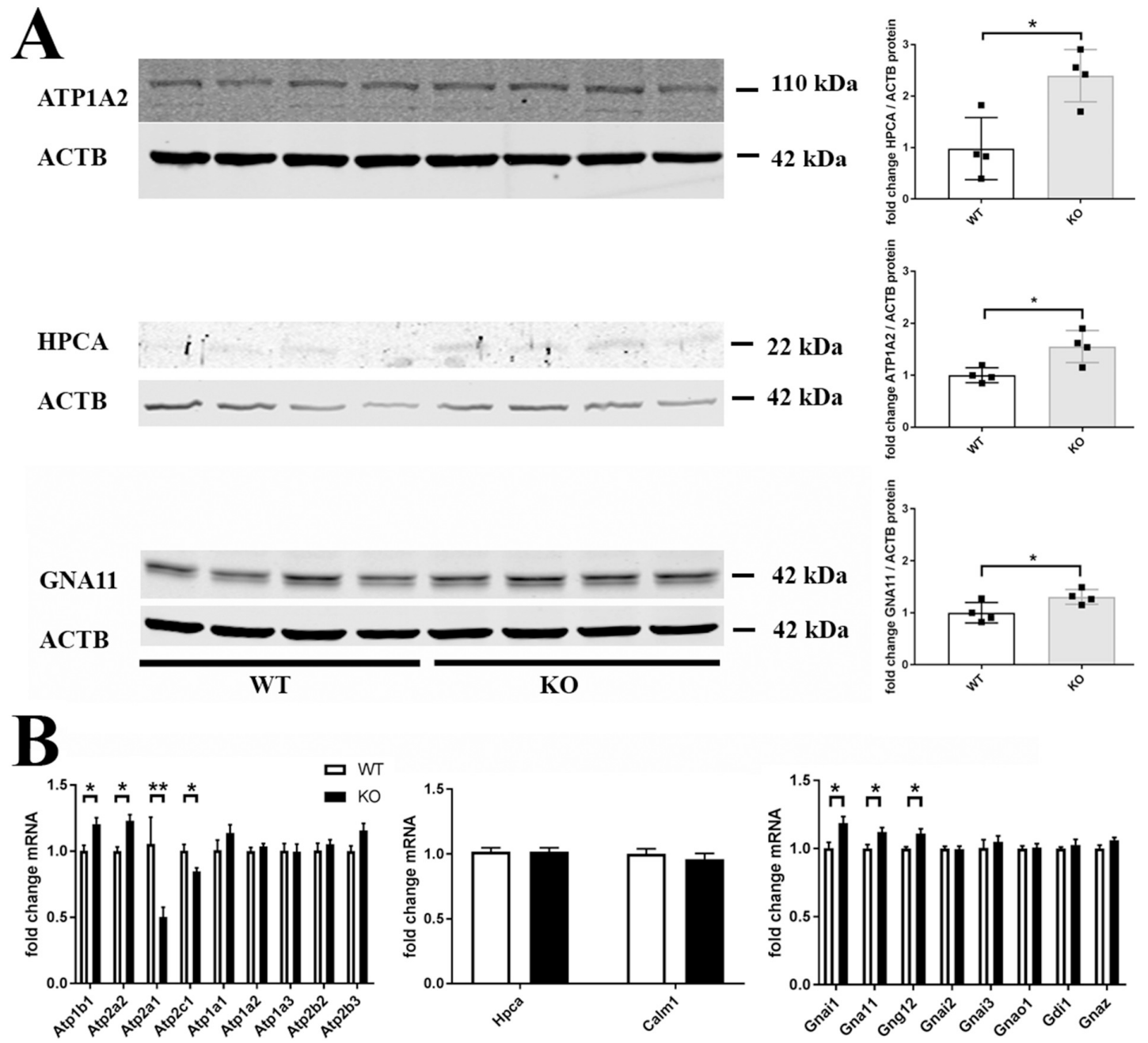

Fig. 3. Validation by analyses of steady-state expression levels in brain tissues from 18-month-old mice. (A) Protein: Quantitative immunoblots showed Hippocalcin and GNA11 to be significantly increased in the RIPA fraction, and ATP1A2 significantly increased in the SDS fraction, while VDAC in the SDS fraction as well as $\mathrm{Ga}(\mathrm{i})$ in the RIPA fraction appeared unchanged (data not shown) ( $n=4$ wildtype vs. 4 mutant mice). Significance was determined with unpaired Student's $t$ test and illustrated in the bar graphs by asterisks $(*=p<.05)$. (B) mRNA: Quantitative reverse transcriptase PCR demonstrated significantly elevated mRNA levels for the ATP-driven ion channels Atp1b1 and Atp2a2, while the expression levels of Atp2a1 and Atp2c1 were reduced. The transcript levels of Hpca and Calm1 appeared unchanged. Levels of the G-protein subunits Gnail and Gna11 and Gng12 were significantly increased ( $n=4$ wildtype vs. 4 mutant mice). Significance was determined with unpaired Student's $t$-test and illustrated in the bar graphs by asterisks $\left.{ }^{*}=p<.05, * *=p<.01\right)$.

2005), but it is unknown whether it triggers compensatory changes in G-protein subunit expression.

\subsubsection{Additional enrichment of calcium-dependent after hyperpolarization factors}

Thus, it appears meaningful that PRMT1 showed decreased ubiquitylation (\$314/324/342-2.3-fold), as a modulator of the Hippocalcin interactor KCNQ (Kim et al., 2016a; Kim et al., 2012), similar to the calcium channel interactor and endocytosis regulator DPYSL2 (CRMP2, - 3.5-fold at §423, - 2.5-fold at §146), which controls the surface transport of KCNQ (Jiang et al., 2015). Further support for the relevance of afterhyperpolarization comes from the observation that HCN2 shows an excessive ubiquitylation ( +2.0 -fold at §510), as a hyperpolarization-activated and cyclic-nucleotide-gated potassium and sodium channel (Ingram and Williams, 1996) that has important pacemaker modifying roles in neurons and heart. HCN contributes to afterhyperpolarization and modulates dopaminergic (D2) modulation of the sAHP in striatal neurons (Deng et al., 2007; Gu et al., 2005). Additional findings are consistent with this context, in particular the excessive ubiquitylation of a peptide from the voltage-gated calcium channel CACNA1B $(+2.1$-fold at $\S 65)$ and other calcium and sodium pumps (ATP2C1 $\S 8+7.2$-fold, ATP1A1 $\S 177+2.2$-fold, ATP2B2 $\S 191+2.2$-fold, ATP2A2 $\S 628+2.1$-fold, but ATP1A1 $\S 36 / 37-2.0$ fold, ATP1B1 §5-2.0-fold, ATP1A2 §505-2.3-fold, ATP1A1/ATP1A3/ ATP1A4 §162/152/172-3.8-fold), some of which are also known modulators of sAHP (Tiwari et al., 2018). Overall, this combined evidence led us to focus on functional tests of axonal slow afterhyperpolarization and action potential firing frequency.

\subsubsection{Enrichment of cGMP-PKG signaling, GTPases, G-proteins}

This enrichment involved not only the factors recognized by bioinformatics and listed in Supplementary Table 1, namely ATP1A1, ATP1A2, ATP1B1, CALM1, GNAI1, PPP3CA, but also the Rab27AGTPase activating protein alpha (TBC1D10A §99/100-3.7-fold), the 
A

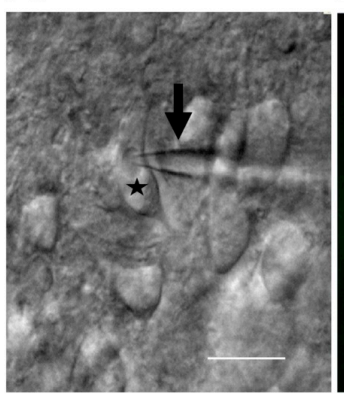

B

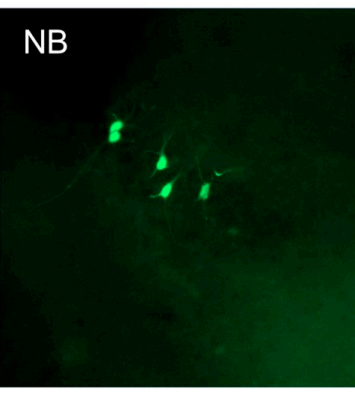

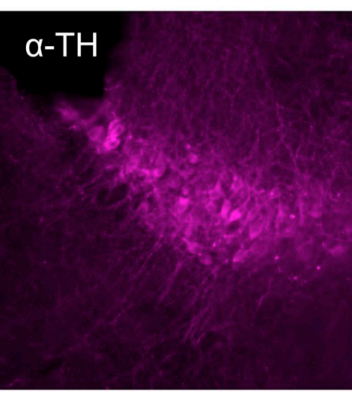

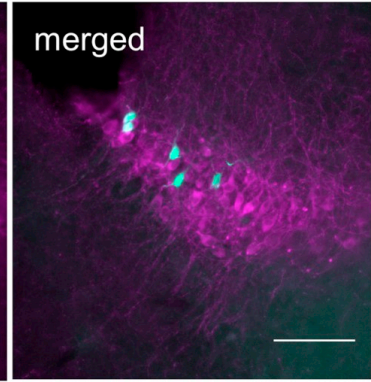

C

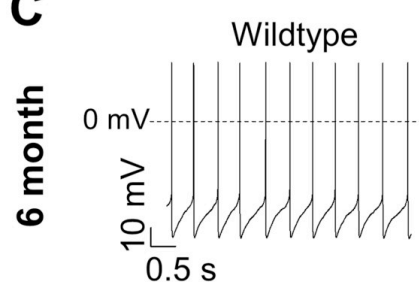

D

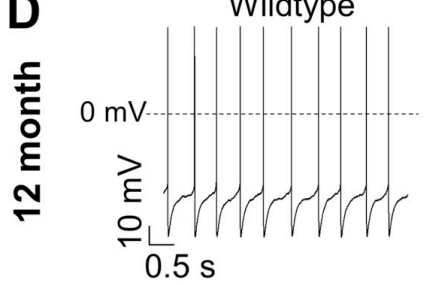

Parkin $\mathrm{KO}$

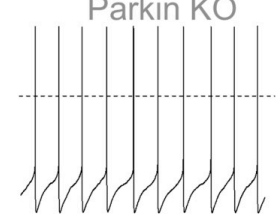

Parkin $\mathrm{KO}$

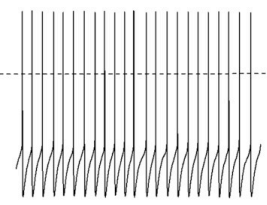

E

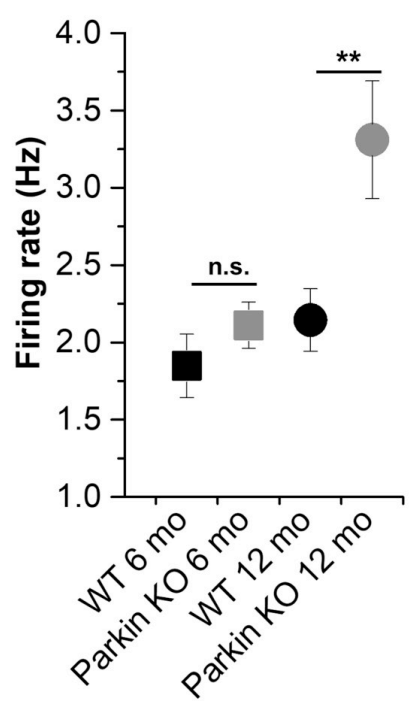

Fig. 4. Analysis of the intrinsic firing patterns of WT and Parkin-KO noradrenergic LC neurons. (A) Representative image of a patch pipette (black arrow) approaching a LC neuron (asterisk). Scale bar: $20 \mu \mathrm{m}$. (B) Co-staining of neurobiotin (NB) filled neurons with Alexa488 conjugated streptavidin (green) and anti-TH/ anti-rabbit Alexa568 (magenta). Scale bar: $200 \mu \mathrm{m}$. (C-D) Example recordings of a spontaneously active WT (black) and Parkin-KO (grey) LC neuron from 6-monthold (upper panel) or 12-month-old (lower panel) mice in the whole-cell current clamp configuration. To isolate autonomous spiking, GABAergic (CGP, gabazine) and glutamatergic (AP-5, NBQX) blockers were added to the bath solution (ACSF). (E) Quantification of the firing rate revealed a significantly higher action potential frequency in the Parkin-KO group at the age of 12 months (WT 6 months: $1.85 \pm 0.20 \mathrm{~Hz}, n=24$; Parkin-KO $6 \mathrm{months:} 2.11 \pm 0.15 \mathrm{~Hz}, n=34$; WT $12 \mathrm{months}$ $2.14 \pm 0.20 \mathrm{~Hz}, n=20$; Parkin-KO 12 months: $3.31 \pm 0.38 \mathrm{~Hz}, n=16$ ). Data are presented as mean \pm S.E.M.; n.s.: not significant; **: $p<.01$ using unpaired Student's $t$-test, as these data followed a normal distribution. (For interpretation of the references to colour in this figure legend, the reader is referred to the web version of this article.)

Smoothened G-protein coupled receptor modulator DDB1 (\$1121-3.7fold), the Rab GDP-dissociation inhibitor GDI1 (§435-2.3-fold), the Gprotein coupled receptor responsiveness modulator EFR3B (\$595/ 639-2.2-fold), the Rho GDP-dissociation inhibitor ARHGDIA ( $\$ 141-2.0$-fold), the G protein subunit GNG12 ( $\$ 34-2.0$-fold), the RAB3A interactor RPH3A ( $\$ 84+2.2$-fold), the RP2 RabGAP interactor CFAP36 ( $\$ 116+2.8$-fold) and interestingly also the Amyloid Beta A4 precursor APP (\$688/728/763 + 4.7-fold), which inhibits G(o) alpha ATPase activity (Ramaker et al., 2013). The known role of Parkin for the mitochondrial GTPases MFN1 and DRP1, the novel strong dysregulation of the neural cGMP modulator Hippocalcin and this additional cGMP-PKG signaling pathway enrichment in old Parkin-KO brain made us conduct a systematic assessment of G-protein subunit expression.

\subsection{Molecular validation by analyses of steady-state expression}

We attempted to elucidate if (1) the ubiquitylation changes influence the steady-state levels of the respective proteins, (2) they are accompanied by compensatory or additive adaptations of mRNA expression, and (3) they trigger homeostatic responses among other pathway components. Given that the ubiquitylome profile (Table 1) quantified peptides from several protein families where dysregulation may reflect only a specific isoform, validation with different approaches was necessary to identify the underlying factors. For this purpose, quantitative immunoblots and RT-qPCR were used in extracts from independent brains. A few commercial antibodies showed sufficient sensitivity and specificity to quantify independent epitopes in the endogenous levels of such proteins against whole brain background. In contrast, for the transcripts of almost all isoforms there were specific commercial assays at the RT-qPCR level. The experiments were focused on the families of ATP-driven $\mathrm{Ca}^{2+}$ pumps and $\mathrm{Na}^{+} / \mathrm{K}^{+}$pumps, Hippocalcin and Calmodulin as well as the G-protein subunits, as novel findings that are important for neural excitation. 


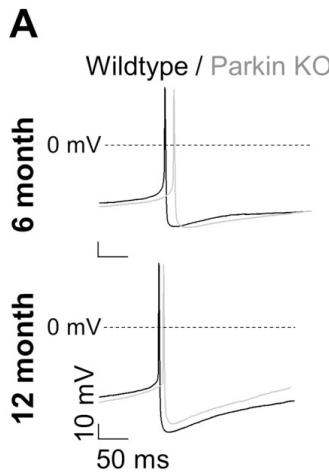

B

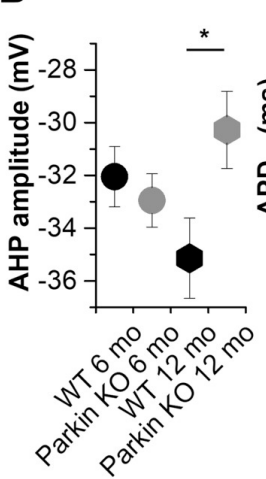

C

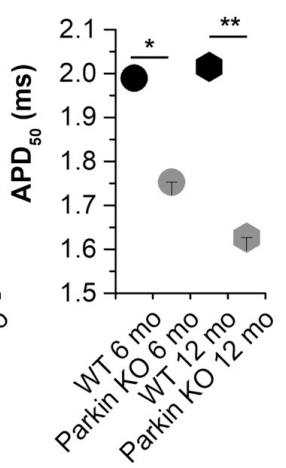

D

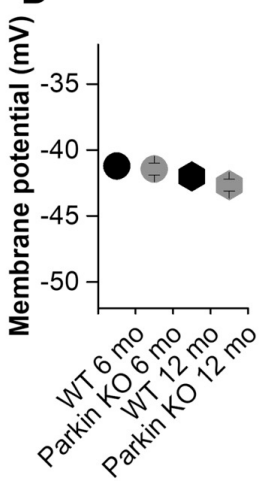

E

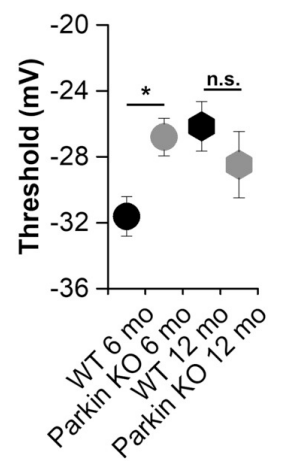

Fig. 5. Analysis of action potential parameters of WT and Parkin-KO LC neurons. (A) Overlay of representative current clamp recordings of WT (black) and Parkin-KO (grey) LC neurons of 6-month-old (upper panel) or 12-month-old (lower panel) mice. (B) Quantification of the afterhyperpolarization (AHP) amplitude revealed a significant decrease in Parkin-KO neurons at the age of 12 months (WT 6 months: $-32.05 \pm 1.14 \mathrm{mV}$; Parkin-KO 6 months: $-32.95 \pm 1.01 \mathrm{mV}$; WT 12 months: $-35.14 \pm 1.52 \mathrm{mV}$; Parkin-KO 12 months: $-30.28 \pm 1.46 \mathrm{mV}$ ). AHP amplitude was defined as the differential between the AP threshold and the most negative potential and AP threshold was defined as the membrane voltage at which APs at high resolution illustrate an abrupt rise. (C) Action potential duration $\left(\mathrm{APD}_{50}\right)$ was reduced in Parkin-KO neurons of mice at the age of 6 and 12 months (WT 6 months: $1.99 \pm 0.09 \mathrm{~ms}$; Parkin-KO 6 months: $1.75 \pm 0.06$ ms; WT 12 months: $2.02 \pm 0.09 \mathrm{~ms}$; Parkin-KO 12 months: $1.63 \pm 0.08$ ). No differences were observed concerning the mean membrane potential (WT 6 months: $-41.21 \pm 0.81 \mathrm{mV}$; Parkin-KO 6 months: $-41.44 \pm 0.46 \mathrm{mV}$; WT 12 months: $-42.00 \pm 0.61 \mathrm{mV}$; Parkin-KO $12 \mathrm{months}$ : $42.67 \pm 0.44 \mathrm{mV}$ ) (D) and the action potential threshold (WT 6 months: $-31.62 \pm 1.19 \mathrm{mV}$; Parkin-KO 6 months: $-26.80 \pm 1.13 \mathrm{mV}$; WT $12 \mathrm{months:}-26.15 \pm 1.51 \mathrm{mV}$; Parkin-KO $12 \mathrm{months}$ : $-28.48 \pm 2.01 \mathrm{mV}$ ) (E). (B-E: WT 6 months, $n=19$; Parkin-KO 6 months, $n=35$; WT 12 months, $\mathrm{n}=20$; Parkin-KO 12 months, $n=15$ ). Data are presented as mean \pm S.E.M. *: $\mathrm{p}<.05 ;{ }^{* *}: \mathrm{p}<.01$ using unpaired Student's t-test, as these data followed a normal distribution.

\subsubsection{At the protein level}

Upon analysis of the steady-state of protein abundance, significantly increased abundance was confirmed (see Fig. 3A) for ATP1A2 (1.55fold, $p=.018$ ), for Hippocalcin (2.4-fold, $p=.011$ ) and for GNA11 (1.3-fold, $p=.045$ ), a key mediator of membrane currents and action potentials in response to extracellular calcium, which can trigger hypocalcemia (Nesbit et al., 2013; Pahlavan et al., 2012; Roszko et al., 2017). In contrast, the levels of the mitochondrial voltage-gated anion channels Porin and the inhibitory G-protein subunit Ga(i) appeared unchanged. Unfortunately, an antibody that targets VDAC3 selectively is not available, the employed anti-Porin antibody recognizes VDAC1-3, with VDAC1 as the most abundant and VDAC3 being the least abundant isoform. Thus, the increased level of VDAC3 protein could not be demonstrated.

\subsubsection{At the mRNA level}

Significantly altered expression was documented (see Fig. 3B) for the ATP-driven $\mathrm{Ca}^{2+}$ pumps Atp2a1 (0.50-fold, $\left.\mathrm{p}=.04\right)$, Atp2a2 (1.23fold, $p=.003$ ), Atp2c1 (0.85-fold, $p=.03$ ) and the ATP-driven $\mathrm{Na}^{+}$/ $\mathrm{K}^{+}$pump Atp1b1 (1.20-fold, $\left.\mathrm{p}=.01\right)$, as well as the G-protein subunits Gnai1 (1.19-fold, $\mathrm{p}=.01$ ), Gna11 (1.12-fold, $p=.02$ ) and Gng12 (1.11fold, $\mathrm{p}=.02)$, while the transcripts for the calcium-sensors Hpca and Calm1, as well as the transcripts for the ion pump Atp1a2 appeared normal. The dysregulation of the endoplasmic reticulum associated SERCA isoforms (Atp2a1 and Atp2a2) appeared prominent; they play a role in the axon initial segment for the generation of axon potentials (Anton-Fernandez et al., 2015; Zhao et al., 2001) and interact with mitochondrial calcium pumps (Surmeier et al., 2012).

\subsubsection{Is increased abundance of GNA11, HPCA and ATP1A2 protein due to turnover or re-synthesis?}

The above findings document that selected factors within pathways of neural signaling and excitability show not only ubiquitylation changes, but also altered protein abundance and/or mRNA expression. Upon synopsis of all data it is usually impossible to predict which changes constitute primary mutation effects with pathogenic consequences, or which other events are homeostatic responses. In the case of the inhibitory G-protein subunit GNAI1 it is clear that its + 5.4-fold elevated ubiquitylation at $\$ 47 / 51$ would decrease its protein steadystate levels, but its -2.2 -fold reduced ubiquitylation at $\$ 330 / 31$ and its increased mRNA levels would oppose this effect, acting to maintain the normal GNAI1 levels that were observed experimentally upon quantitative immunoblotting. In the case of the phospholipase $\mathrm{C}$ activating subunit GNA11, significantly elevated protein levels were documented and appear to be due to a transcriptional upregulation effort. Regarding HPCA in contrast, its increased protein levels appear to be caused by the decreased protein degradation (the -4.7 -fold decrease of ubiquitylation at §137) in view of its unchanged mRNA expression, and would act to enhance slow afterhyperpolarization after axonal action potentials. It is not clear whether this dysregulation is a pathological or a compensatory event. Considering ATP1A2, its elevated protein levels seem to be simply due to decreased ubiquitylation ( -2.3 -fold at §505) in view of its unchanged mRNA expression. Thus, both Hippocalcin and ATP1A2 might represent novel Parkin substrates.

\subsection{Effects of Parkin-KO on neuronal electrophysiology}

\subsubsection{Focusing on brainstem noradrenergic signals}

It remained unclear, which molecular changes represent primary pathology, which dysregulations are compensating a problem over some time, and how they progressively impair neural function. It was previously reported that these mice show discrete deficits of hippocampal long-term potentiation, spatial memory and open-field habituation (Rial et al., 2014). To elucidate these questions further, we undertook the laborious assessment of electrophysiology of activation pathways in acute brain slices. In view of the previously reported preferential vulnerability of the brainstem locus coeruleus (LC) in ParkinKO brains (Von Coelln et al., 2004), our work attempted to find maximal phenotypes, thus focusing on LC neurons that serve in noradrenergic G-protein coupled receptor signaling, rather than the midbrain dopaminergic substantia nigra (SN) neurons or hippocampus. To take homeostatic efforts at earlier ages versus manifestation of phenotypes at later ages into account, 6-month-old versus 12-month-old mutant and wildtype mice derived from the same ancestors and maintained in adjacent cages under identical conditions were assessed. To investigate axonal firing patterns in acute brainstem slices for 
J. Key, et al.

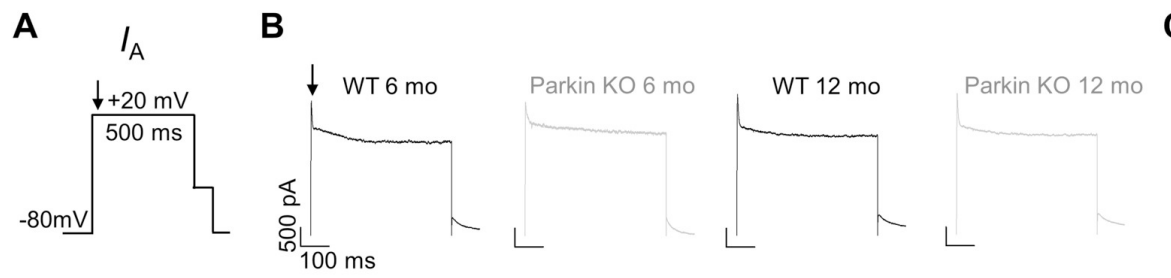

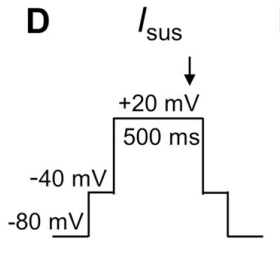

E

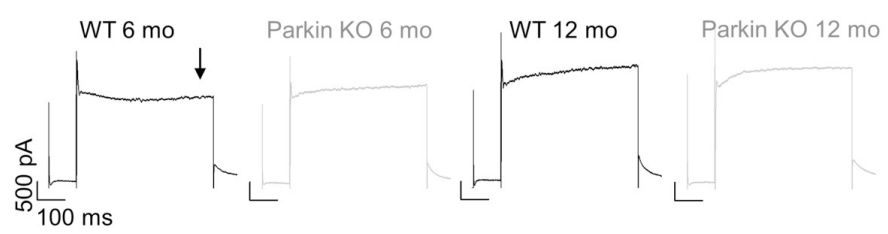

G

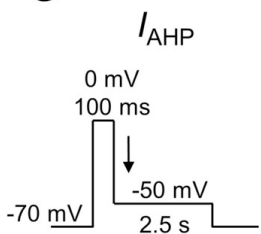<smiles>[AlH2]</smiles>

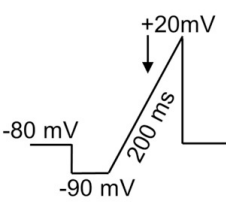

H

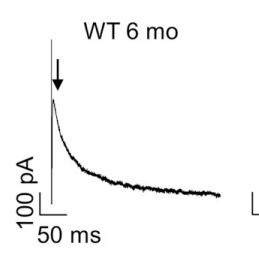

K
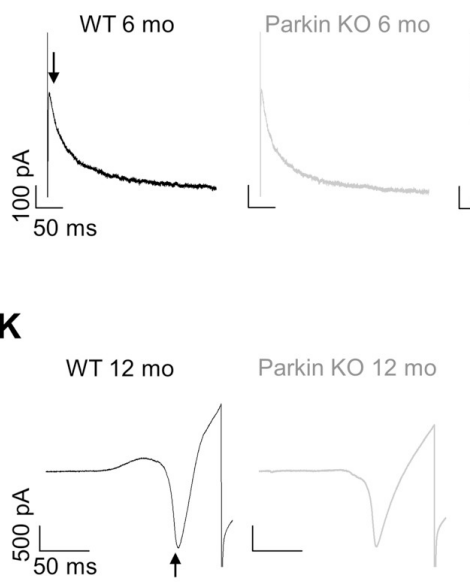

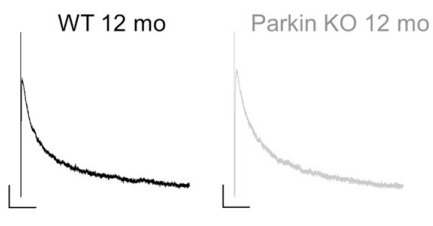

L

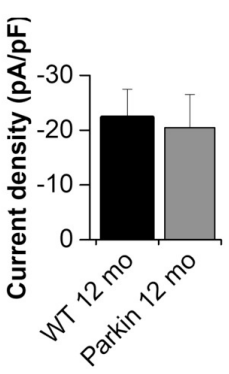

\section{$\mathrm{C}_{\overline{\mathrm{L}}}$}

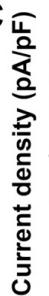

$F$ 뜽
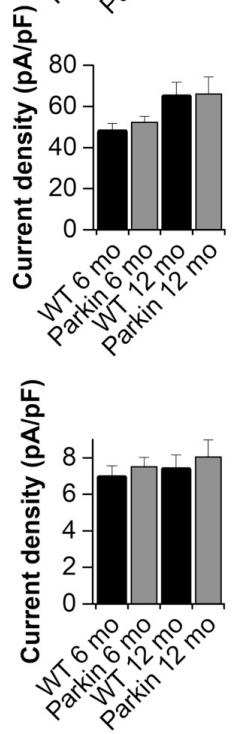

Fig. 6. Comparison of $\mathrm{K}^{+}$and $\mathrm{Ca}^{2+}$ currents in LC neurons of WT and Parkin-KO mice. (A-C) Analysis of the rapidly inactivating component of the $\mathrm{K}^{+}$outward current $\left(I_{\mathrm{A}}\right)$ at $20 \mathrm{mV}$ obtained with the voltage step protocol depicted in (A). As indicated by arrows peak current was analyzed directly at the beginning of the voltage step to $20 \mathrm{mV}$. (B) Representative whole-cell voltage clamp recordings. (C) Quantification of $I_{\mathrm{A}}$ did not reveal any significant difference between the groups (WT 6 months: $59.43 \pm 48 \mathrm{pA} / \mathrm{pF}, n=17$; Parkin-KO 6 months: $68.65 \pm 3.23 \mathrm{pA} / \mathrm{pF}, n=30$; WT 12 months: $71.69 \pm 6.58$, $\mathrm{n}=19$; Parkin-KO 12 months: $65.28 \pm 4.11 \mathrm{pA} / \mathrm{pF}, n=21)$. (D-F) Analysis of the sustained component of the $\mathrm{K}^{+}$outward current $\left(I_{\text {sus }}\right)$ at $20 \mathrm{mV}$ obtained with a voltage step protocol comprising a pre-pulse to $-40 \mathrm{mV}$ as displayed in (D). (E) Representative whole-cell voltage clamp recordings. (F) Quantification of $I_{\text {sus }}(\mathrm{WT} 6 \mathrm{months:} 47.70 \pm 4.06 \mathrm{pA} / \mathrm{pF}$, $\mathrm{n}=17$; Parkin-KO 6 months: $52.31 \pm 2.80 \mathrm{pA} / \mathrm{pF}, n=31$; WT 12 months: $64.66 \pm 7.13 \mathrm{pA} / \mathrm{pF}, \mathrm{n}=19$; Parkin-KO $12 \mathrm{months:} 66.10 \pm 8.22 \mathrm{pA} / \mathrm{pF}, \mathrm{n}=21)$. (GI) Analysis of the $\mathrm{K}^{+}$current flowing during the afterhyperpolarization $\left(I_{\mathrm{AHP}}\right)$. AHP currents were evoked by the two-step voltage protocol depicted in (G) and analyzed at the step to $-50 \mathrm{mV}$. (H) Representative whole-cell voltage clamp recording of AHP currents. (I) Mean AHP current densities were: WT 6 months: $6.92 \pm 0.66 \mathrm{pA} / \mathrm{pF}, \mathrm{n}=17$; Parkin-KO 6 months: $7.51 \pm 0.52 \mathrm{pA} / \mathrm{pF}, \mathrm{n}=30$; WT 12 months: $7.35 \pm 0.80 \mathrm{pA} / \mathrm{pF}, \mathrm{n}=17$ and Parkin-KO 12 months: $8.05 \pm 0.92$ $\mathrm{pA} / \mathrm{pF}, \mathrm{n}=19$ ). (J-L) Analysis of peak $\mathrm{Ca}^{2+}$ inward currents obtained with the voltage ramp shown in (J) and using a cesium based internal solution. ACSF was complemented with $1 \mu \mathrm{M}$ TTX. (K) Representative whole-cell voltage clamp recording of $\mathrm{Ca}^{2+}$ inward currents. (L) Analysis of peak Ca ${ }^{2+}$ inward currents did not reveal a significant difference between WT and Parkin-KO (WT 12 months: $-22.26 \pm 5.23 \mathrm{pA} / \mathrm{pF}, \mathrm{n}=4$; Parkin-KO 12 months: $-20.43 \pm 6.07 \mathrm{pA} / \mathrm{pF}, n=5$ ). Data are presented as mean \pm S.E.M.

murine LC neurons, which were histologically verified as previously described (Henrich et al., 2018; Matschke et al., 2015; Matschke et al., 2018) (Fig. 4A-B), whole-cell current clamp and voltage clamp experiments were conducted (Figs. 4-7).

3.6.2. Progressive alteration of the intrinsic pacemaking frequency The intrinsic firing pattern of LC neurons recorded in the whole cell current clamp mode showed a significantly higher action potential frequency in Parkin-KO mice at the age of 12 months (Fig. 4C-E). In parallel, the afterhyperpolarization (AHP) amplitude was significantly decreased at this age (Fig. 5A-B), an interesting finding in view of the influence of Hippocalcin on sAHP (Kim et al., 2012; Larsson, 2013; Tzingounis et al., 2007). Already at the age of 6 months and again at 12 months, the action potential duration $\left(\mathrm{APD}_{50}\right)$ was significantly 
A

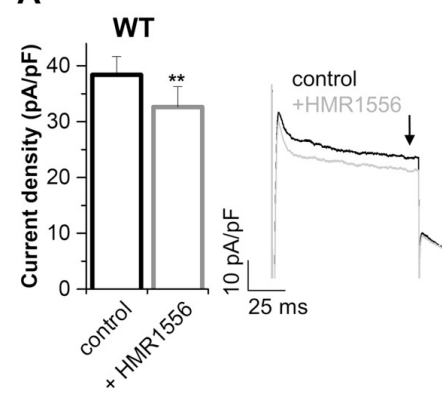

B

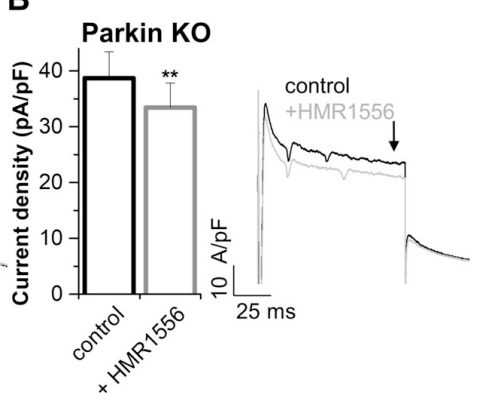

C

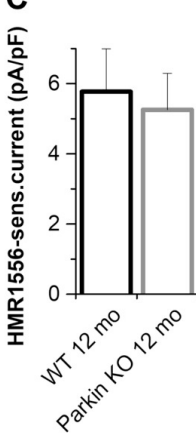

Fig. 7. Analysis of KCNO mediated currents in LC neurons of 12-month-old WT and Parkin-KO mice. It is known that the calcium buffer protein Hippocalcin, which shows an altered turnover in Parkin-KO mice, interacts with the $\mathrm{Ca}^{2+}$ sensor of KCNQ channels, thereby modulating the slow AHP current (Kim et al., 2012). We therefore tested whether KCNQ mediated currents are altered in 12-month-old Parkin-KO mice using the KCNQ channel blocker HMR1556. Currents were evoked with the same voltage clamp protocol as shown in Fig. 6 D. (A-B) Analysis of sustained $\mathrm{K}^{+}$outward currents (left panels) and sample traces of evoked currents (right panels) of WT (A) and Parkin-KO (B) LC neurons at $0 \mathrm{mV}$ before (control) and after wash in of $1 \mu \mathrm{M}$ HMR1556. In both groups drug application induced a significant decrease of the $\mathrm{K}^{+}$outward current (WT control: $38.38 \pm 3.26 \mathrm{pA} / \mathrm{pF}, n=6$; WT + HMR1556: $32.61 \pm 3.70 \mathrm{pA} / \mathrm{pF}$, $\mathrm{n}=6$; Parkin-KO control: $38.71 \pm 4.73$ pA7pF, $n=7$; Parkin-KO + HMR1556: $33.45 \pm 4.35 \mathrm{pA} / \mathrm{pF}, \mathrm{n}=7$ ). (C) Comparison of the HMR1556 sensitive current did not reveal a significant difference between WT and Parkin-KO mice (WT 12 months: $5.77 \pm 1.22 \mathrm{pA} / \mathrm{pF}$; Parkin-KO 12 months: $5.26 \pm 1.04 \mathrm{pA} / \mathrm{pF}$ ). Data are presented as mean \pm S.E.M. **: $\mathrm{p}<.01$ using unpaired Student's t-test, as these data followed a normal distribution.

reduced (Fig. 5C), while the mean membrane potential and the action potential threshold appeared normal (Fig. 5D-E).

\subsubsection{Underlying mechanism not explained by distinct changes in ionic} currents across the plasma membrane

To investigate whether the altered firing behavior of aged ParkinKO LC neurons is reflected by changes of distinct ionic conductances, we subsequently used different voltage clamp protocols to isolate $\mathrm{K}^{+}$ and $\mathrm{Ca}^{2+}$ currents across the plasma membrane. The study of the $I_{\mathrm{A}}$ (Fig. 6A-C) and $I_{\text {sus }}$ (Fig. 6D-F) $\mathrm{K}^{+}$currents revealed no significant differences in macroscopic potassium flux in Parkin-KO LC neurons, both, at the age of 6 and 12 months. Also the $I_{\mathrm{AHP}}$ (Fig. 6G-I) and the $\mathrm{Ca}^{2+}$ currents (Fig. 6J-L) regulating the afterhyperpolarization in LC neurons (Matschke et al., 2018) were not significantly altered in the LC neurons of Parkin-KO mice. Given that Hippocalcin is known to modulate SAHP via its interaction with the calcium sensor of KCNQ (Kim et al., 2012) and that the KCNQ-interactors PRMT1 and DPYSL2 showed decreased ubiquitylation, the KCNQ-specific antagonist HMR1556 was used to isolate KCNQ-mediated component of the $I_{\text {sus }}$ current (Fig. 7A-C). However, we did not identify significantly different HMR1556-sensitive current components in 12-month-old Parkin-KO mice (Fig. 7C), suggesting that KCNQ channels are not functionally altered. Thus, the AHP amplitude is altered in Parkin-KO mice while in whole cell voltage-clamp recordings with a 'clamped' $\mathrm{Ca}^{2+}$ concentration, the $I_{\mathrm{AHP}}$ and $\mathrm{Ca}^{2+}$ currents are not altered. These negative findings are consistent with the concept that the alterations in AHP, $\mathrm{APD}_{50}$ and action potential frequency are consequences of anomalies in calcium homeostasis and other ion membrane pumps and not by altered expression levels or functional properties of $\mathrm{K}^{+}$or $\mathrm{Ca}^{2+}$ channels at the plasma membrane.

\section{Discussion}

To understand Parkin-dependent protein post-translational modifications in whole brain and their functional consequences in noradrenergic LC neurons, we undertook a global ubiquitylome survey and validation work by immunoblots, qPCR and electrophysiology. As expected, the strongest Parkin-dependent ubiquitylation deficit was observed for mitochondrial outer membrane protein VDAC3, providing proof-of-principle that our approach is consistent with previous work on peripheral tumor cell lines like HeLa. Several novel ubiquitylation deficits were identified in protein complexes and pathways that are under control of Parkin, so TBC1D10A, SIRT2, OTUD7B and CUL5 may represent direct Parkin targets, but this has to be assessed further in overexpression and co-immunoprecipitation experiments. In this manuscript, we maintained the focus on neuron-specific factors that influence axonal excitability, based on the bioinformatics enrichment of dysregulations among ion channels, calcium homeostasis and G-protein dependent signaling.

As a novel candidate Parkin substrate, ATP1A2 showed increased abundance due to reduced ubiquitylation at residue 505 in absence of transcriptional regulation. Relatively little is known about ATP1A2 in the nervous system. According to studies that relied on the monoclonal antibody McB2 (Cholet et al., 2002), at adult age the main brain expression of ATP1A2 occurs in astrocytes, but it may be important to note that adult mouse brain shows a predominant neural expression of Atp1a2 mRNA according to the internet database Allen mouse brain atlas (http://mouse.brain-map.org/). ATP1A2 deficiency leads to reduced burst activity in neurons (Moseley et al., 2003) and modulates calcium dynamics (Despa et al., 2012; Hartford et al., 2004). Mutations in ATP1A2 trigger the pathogenesis of migraine in interaction with voltage-gated calcium channels (Lafreniere and Rouleau, 2011).

Hippocalcin is a $\mathrm{Ca}^{2+}$ sensor of a variant form of the ROS-GC subfamily of membrane guanylate cyclases that senses physiological increments of $\mathrm{Ca}^{2+}$ and stimulates ONE-GC membrane guanylate cyclase, controlling about $30 \%$ of the total membrane guanylate cyclase transduction system (Krishnan et al., 2009). In this way, it modulates Gprotein coupled receptor signals and second messenger cascades (Mammen et al., 2004; Sallese et al., 2000). Its mRNA is expressed strongly in pyramidal cells of the hippocampus and in Purkinje neurons of the cerebellum, but it is found also in cerebral cortex neurons and in large neurons of the striatum (Saitoh et al., 1993). It appears to be particularly crucial for the dopaminergic nigrostriatal projections, since its mutation triggers a phenotype of primary isolated dystonia, named DYT2 (Charlesworth et al., 2015). Its expression downregulation accompanies the onset of Huntington's disease, where striatal neurodegeneration with motor hyperactivity are the prominent features (LuthiCarter et al., 2002; Luthi-Carter et al., 2000). Hippocalcin deficiency impairs the neuronal responses of calcium-dependent excitation to glutamate (Charlesworth et al., 2015). The interaction between muscarinic acetylcholine receptors and glutamatergic NMDA receptors in the control of long-term depression depends on Hippocalcin, so postsynaptic excitability is modulated by it (Amici et al., 2009; Jo et al., 2010; Palmer et al., 2005). Hippocalcin binds to brain-type creatine kinase and modulates its calcium-dependent translocation to membranes (Kobayashi et al., 2012). Importantly, it also binds to the neuronal apoptosis inhibitory protein (NAIP). The HPCA-NAIP complex protects neurons from mitochondrial dysfunction, counteracting pathological calcium release and caspase activation (Mercer et al., 2000). Diverse NAIP isoforms are crucial in eukaryotic cells for the detection of 
invading bacterial proteins, activation of the inflammasome and of pyroptosis (Amarante-Mendes et al., 2018; Kofoed and Vance, 2011; Reyes Ruiz et al., 2017; Zhao et al., 2011). In the Parkin-KO mouse brain, we observed increased levels of Hippocalcin together with decreased ubiquitylation, a dysregulation that might be expected to enhance sAHP after axonal action potentials (Tzingounis et al., 2007). Our electrophysiological data, however, documented diminished sAHP in aged Parkin-KO. Thus, the reduced ubiquitylation of HPCA might not constitute a direct Parkin-KO effect, but rather an indirect compensatory neuronal effort to rescue the sAHP that is abnormally low due to the calcium dysregulations.

Although it was not identified as a target of Parkin-dependent ubiquitylation changes, GNA11 (Go11) stands out for its selective transcriptional induction, which achieves a steady-state elevation of its protein levels. Ga11 does not permanently reside at plasma membranes, but can relocate to the outer mitochondrial membrane (in parallel to Gaq relocation to the mitochondrial inner membrane) where it is responsible for the proper equilibrium between fusion and fission (Beninca et al., 2014), which is maintained via altered levels of both mitofusin and Drp1 proteins. The GTPases mitofusin 1/2 and DRP1 are known as Parkin substrates, and in our study MFN1 showed reduced ubiquitylation in brain, therefore GTP turnover at the outer mitochondrial membrane might be altered. The absence of Ga11 was reported to decrease mitochondrial fusion rates and overall respiratory capacity, ATP production and OXPHOS-dependent growth (Beninca et al., 2014), so the induction of Ga11 in Parkin-KO brain might have the opposite neuroprotective effects. Importantly, Ga11 and $\mathrm{G \alpha q}$ modulate also $\mathrm{Ca}^{2+}$ release and $\mathrm{Ca}^{2+}$ entry in excitable cells (MacrezLepretre et al., 1997). At the same time, Ga11 and Gaq control neuronal excitability via the PLC $\beta$ signaling cascade and the calcium-sensitive, potassium-dependent afterhyperpolarizing current, which is modified by Hippocalcin and Neurocalcin delta (Krause et al., 2002; Madison and Nicoll, 1984; Pedarzani and Storm, 1993; Schwindt et al., 1988; Villalobos and Andrade, 2010). Thus, myocytes from the Gaq and/or Ga11 KO mice showed altered action potentials, membrane currents, and $\mathrm{Ca}^{2+}$ handling (Pahlavan et al., 2012). Cerebellar Purkinje and hippocampal pyramidal neurons from the Gaq and/or Ga11 KO mice showed altered synaptic plasticity and motor behavior (Hartmann et al., 2004; Miura et al., 2002). Therefore, the upregulation of Ga11 in Parkin-KO brain provides an interesting insight, how brain cells couple the mitochondrial function with calcium-dependent excitability and firing frequency regulation at the plasma membrane.

In conclusion, our pioneer study of the Parkin-KO brain ubiquitylome has identified several novel peptides and residues that show deficient posttranslational modifications. Two neuron-specific factors among them, ATP1A2 and Hippocalcin, show increased abundance in the absence of transcriptional activation. Both are known modulators of calcium-dependent action potential generation and afterhyperpolarization and indeed significant changes in these electrophysiological features were documented in noradrenergic LC neurons. Overall, the progressive changes in neuronal firing correlate well to the strong ubiquitylation decrease for several sodium/potassium-transporting ATPases (ATP1A1/2/3/4) and the strong ubiquitylation increase for ATP2C1 that sequestrates calcium to the secretion pathway (Brini et al., 2012). The increased neuronal excitability may also relate to the decreased ubiquitylation of protein kinase M (PKM), which was previously identified as possible Parkin substrate and which acts in calcium-dependent manner to induce burst firing in dopaminergic neurons (Liu et al., 2007; Sarraf et al., 2013). Interestingly, the action potential duration was already reduced at Parkin-KO ages of 6 months, whereas firing frequency and sAHP alterations were clearly abnormal only by 12 months of age. Investigations of the preferential vulnerability of brainstem and midbrain catecholaminergic neurons to Parkinsonian neurodegeneration have shown that they have an outstanding dependence of their pacemaker activity on L-type calcium channels at the plasma membrane (Matschke et al., 2015; Surmeier et al., 2012). The stimulus-dependent changes in their firing frequency require calcium homeostasis changes at mitochondria and endoplasmic reticulum, and it has been reported already that Parkin-deficiency may impair mitochondrial $\mathrm{Ca}^{2+}$ transients (Cali et al., 2014). It is also known that the degeneration of dopaminergic neurons in Pink $1^{-/-}$ zebrafish can be rescued by inhibition of the mitochondrial calciumuptake uniporter MCU (Soman et al., 2017). The increased excitability and altered afterhyperpolarization due to altered mitochondrial $\mathrm{Ca}^{2+}$ homeostasis, which we observed in Parkin-KO mice, appear to be common features in PD animal models. Similar findings were made in mice with Pink $1^{-/-}$genotypes (Bishop et al., 2010; Huang et al., 2017), where a strong transcriptional downregulation was observed for Cisd2 as a factor that drives calcium transport from mitochondria to the endoplasmic reticulum (Gispert et al., 2015; Rouzier et al., 2017; Shen et al., 2017). Furthermore, mice with A53T-alpha-synuclein overexpression show increased firing frequencies in dopaminergic SN neurons, and mice with exposure to Parkinson-inducing drugs exhibit degeneration of dopaminergic $\mathrm{SN}$ neurons in dependence on mitochondrial K-ATP channels (Liss et al., 2005; Subramaniam et al., 2014). It is important to note that several drugs are now available that modulate the activity of mitochondrial VDAC, and that the drug isradipine protects dopaminergic SN neurons via modulation of mitochondrial dynamics and L-type calcium channels (Ben-Hail et al., 2016; Guzman et al., 2018). Future analyses are needed to distinguish direct from indirect Parkin effects, and understand why initial compensatory efforts lose their efficiency during brain ageing. Given that presymptomatic homozygous and compound heterozygous Parkin mutation carriers exhibit an increased frequency of depression (Reetz et al., 2008; Srivastava et al., 2011), and that PARK2 gene microduplications were identified in attention-deficit/hyperactivity disorder (ADHD) patients (Dalla Vecchia et al., 2019), we believe that the mechanisms of Parkin-dependent neural excitability have wide clinical relevance.

Supplementary data to this article can be found online at https:// doi.org/10.1016/j.nbd.2019.02.008.

\section{Acknowledgements}

We thank Birgitt Meseck-Selchow and Gabriele Köpf for technical assistance, and the animal care team at the ZFE Frankfurt (in particular the veterinarians Dr. Alf Theisen and Dr. Christa Tandi, as well as the caretakers Eleonora Daut and Birgit Janton). The study was financed with funds of the University Hospitals in Frankfurt and Marburg, and by the German Ministry of Health with the National Genome Research Network (NGFNplus, BMBF 01GS08138) and the GerontoMitoSys Network (BMBF PTJ 0315584A), and by the European Union through ERAnet-RePARK (DLR 01EW1012).

\section{Competing interests}

The authors declare that there is no competing interest regarding the publication of this manuscript.

\section{References}

Aguilera, M., et al., 2000. Ariadne-1: a vital Drosophila gene is required in developmen and defines a new conserved family of ring-finger proteins. Genetics 155, 1231-1244. Amarante-Mendes, G.P., et al., 2018. Pattern recognition receptors and the host cell death molecular machinery. Front. Immunol. 9, 2379.

Amici, M., et al., 2009. Neuronal calcium sensors and synaptic plasticity. Biochem. Soc. Trans. 37, 1359-1363.

Anton-Fernandez, A., et al., 2015. Selective presence of a giant saccular organelle in the axon initial segment of a subpopulation of layer V pyramidal neurons. Brain Struct. Funct. 220, 869-884.

Antonioli, M., et al., 2014. AMBRA1 interplay with cullin E3 ubiquitin ligases regulates autophagy dynamics. Dev. Cell 31, 734-746.

Azarashvili, T., et al., 2009. Ca2+-dependent permeability transition regulation in rat brain mitochondria by $2^{\prime}, 3^{\prime}$-cyclic nucleotides and 2',3'-cyclic nucleotide $3^{\prime}$-phosphodiesterase. Am. J. Phys. Cell Phys. 296, C1428-C1439. 
Ben-Hail, D., et al., 2016. Novel compounds targeting the mitochondrial protein VDAC1 inhibit apoptosis and protect against mitochondrial dysfunction. J. Biol. Chem. 291, 24986-25003.

Beninca, C., et al., 2014. A new non-canonical pathway of Galpha(q) protein regulating mitochondrial dynamics and bioenergetics. Cell. Signal. 26, 1135-1146.

Bertolin, G., et al., 2013. The TOMM machinery is a molecular switch in PINK1 and PARK2/PARKIN-dependent mitochondrial clearance. Autophagy 9, 1801-1817.

Birsa, N., et al., 2014. Lysine 27 ubiquitination of the mitochondrial transport protein Miro is dependent on serine 65 of the parkin ubiquitin ligase. J. Biol. Chem. 289, 14569-14582.

Bishop, M.W., et al., 2010. Hyperexcitable substantia nigra dopamine neurons in PINK1 and HtrA2/Omi-deficient mice. J. Neurophysiol. 104, 3009-3020.

Bohgaki, M., et al., 2008. Involvement of Ymer in suppression of NF-kappaB activation by regulated interaction with lysine-63-linked polyubiquitin chain. Biochim. Biophys. Acta $1783,826-837$.

Brini, M., et al., 2012. Calcium pumps: why so many? Comprehensive Physiol. 2, 1045-1060.

Cali, T., et al., 2014. Calcium signaling in Parkinson's disease. Cell Tissue Res. 357, 439-454.

Carron, R., et al., 2014. Early hypersynchrony in juvenile PINK1(-)/(-) motor cortex is rescued by antidromic stimulation. Front. Syst. Neurosci. 8, 95 .

Cavadini, S., et al., 2016. Cullin-RING ubiquitin E3 ligase regulation by the COP9 signalosome. Nature 531, 598-603.

Charlesworth, G., et al., 2015. Mutations in HPCA cause autosomal-recessive primary isolated dystonia. Am. J. Hum. Genet. 96, 657-665.

Chin, J.H., et al., 2006. Beta-amyloid enhances intracellular calcium rises mediated by repeated activation of intracellular calcium stores and nicotinic receptors in acutely dissociated rat basal forebrain neurons. Brain Cell Biol. 35, 173-186.

Cholet, N., et al., 2002. Similar perisynaptic glial localization for the $\mathrm{Na}+, \mathrm{K}+$-ATPase alpha 2 subunit and the glutamate transporters GLAST and GLT-1 in the rat somatosensory cortex. Cereb. Cortex 12, 515-525.

Corti, O., et al., 2011. What genetics tells us about the causes and mechanisms of Parkinson's disease. Physiol. Rev. 91, 1161-1218.

Dalla Vecchia, E., et al., 2019. Cross-species models of attention-deficit/hyperactivity disorder and autism spectrum disorder: lessons from CNTNAP2, ADGRL3, and PARK2. Psychiatr. Genet. 29, 1-17.

Dehorter, N., et al., 2012. Subthalamic lesion or levodopa treatment rescues giant GABAergic currents of PINK1-deficient striatum. J. Neurosci. 32, 18047-18053.

Deng, P., et al., 2007. Involvement of $\mathrm{I}(\mathrm{h})$ in dopamine modulation of tonic firing in striatal cholinergic interneurons. J. Neurosci. 27, 3148-3156.

Despa, S., et al., 2012. $\mathrm{Na}(+) / \mathrm{K}(+)$-ATPase alpha2-isoform preferentially modulates $\mathrm{Ca} 2(+)$ transients and sarcoplasmic reticulum $\mathrm{Ca} 2(+)$ release in cardiac myocytes. Cardiovasc. Res. 95, 480-486.

Fournier, M., et al., 2009. Parkin deficiency delays motor decline and disease manifestation in a mouse model of synucleinopathy. PLoS One 4, e6629.

Gautier, C.A., et al., 2016. The endoplasmic reticulum-mitochondria interface is perturbed in PARK2 knockout mice and patients with PARK2 mutations. Hum. Mol. Genet. 25, 2972-2984.

Gegg, M.E., et al., 2010. Mitofusin 1 and mitofusin 2 are ubiquitinated in a PINK1/parkindependent manner upon induction of mitophagy. Hum. Mol. Genet. 19, 4861-4870.

Gehrke, S., et al., 2015. PINK1 and parkin control localized translation of respiratory chain component mRNAs on mitochondria outer membrane. Cell Metab. 21, 95-108.

Geisler, S., et al., 2010. PINK1/parkin-mediated mitophagy is dependent on VDAC1 and p62/SOSTM1. Nat. Cell Biol. 12, 119-131.

Girirajan, S., et al., 2008. Tom112 hypomorphic mice exhibit increased incidence of infections and tumors and abnormal immunologic response. Mamm. Genome 19, $246-262$

Gispert, S., et al., 2009. Parkinson phenotype in aged PINK1-deficient mice is accompanied by progressive mitochondrial dysfunction in absence of neurodegeneration. PLoS One 4, e5777.

Gispert, S., et al., 2015. Potentiation of neurotoxicity in double-mutant mice with Pink1 ablation and A53T-SNCA overexpression. Hum. Mol. Genet. 24, 1061-1076.

Gu, N., et al., 2005. Kv7/KCNQ/M and HCN/h, but not KCa2/SK channels, contribute to the somatic medium after-hyperpolarization and excitability control in CA1 hippocampal pyramidal cells. J. Physiol. 566, 689-715.

Guzman, J.N., et al., 2018. Systemic isradipine treatment diminishes calcium-dependent mitochondrial oxidant stress. J. Clin. Invest. 128, 2266-2280.

Hammerling, B.C., et al., 2017a. A Rab5 endosomal pathway mediates parkin-dependent mitochondrial clearance. Nat. Commun. 8, 14050

Hammerling, B.C., et al., 2017b. Isolation of Rab5-positive endosomes reveals a new mitochondrial degradation pathway utilized by BNIP3 and parkin. Small GTPases 1-8.

Hanson, J.E., et al., 2010. Altered hippocampal synaptic physiology in aged parkin-deficient mice. NeuroMolecular Med. 12, 270-276.

Hartford, A.K., et al., 2004. Na,K-ATPase alpha 2 inhibition alters calcium responses in optic nerve astrocytes. Glia 45, 229-237.

Hartmann, J., et al., 2004. Distinct roles of Galpha(q) and Galpha11 for Purkinje cell signaling and motor behavior. J. Neurosci. 24, 5119-5130.

Helassa, N., et al., 2017. Biophysical and functional characterization of hippocalcin mutants responsible for human dystonia. Hum. Mol. Genet. 26, 2426-2435.

Henrich, M.T., et al., 2018. A53T-alpha-synuclein overexpression in murine locus coeruleus induces Parkinson's disease-like pathology in neurons and glia. Acta Neuropathol. Commun. 6, 39.

Huang, E., et al., 2017. PINK1-mediated phosphorylation of LETM1 regulates mitochondrial calcium transport and protects neurons against mitochondrial stress. Nat. Commun. 8, 1399.
Huber, A.B., et al., 1999. Metalloprotease MP100: a synaptic protease in rat brain. Brain Res. 837, 193-202.

Imai, Y., Takahashi, R., 2004. How do Parkin mutations result in neurodegeneration? Curr. Opin. Neurobiol. 14, 384-389.

Ingram, S.L., Williams, J.T., 1996. Modulation of the hyperpolarization-activated current (Ih) by cyclic nucleotides in Guinea-pig primary afferent neurons. J. Physiol. 492, 97-106 Pt 1.

Itier, J.M., et al., 2003. Parkin gene inactivation alters behaviour and dopamine neurotransmission in the mouse. Hum. Mol. Genet. 12, 2277-2291.

Jiang, L., et al., 2015. Activation of $\mathrm{m} 1$ muscarinic acetylcholine receptor induces surface transport of KCNQ channels through a CRMP-2-mediated pathway. J. Cell Sci. 128, 4235-4245.

Jo, J., et al., 2010. Muscarinic receptors induce LTD of NMDAR EPSCs via a mechanism involving hippocalcin, AP2 and PSD-95. Nat. Neurosci. 13, 1216-1224.

Johnson, B.N., et al., 2012. The ubiquitin E3 ligase parkin regulates the proapoptotic function of Bax. Proc. Natl. Acad. Sci. U. S. A. 109, 6283-6288.

Katz, M., et al., 2002. Ligand-independent degradation of epidermal growth factor receptor involves receptor ubiquitylation and $\mathrm{Hgs}$, an adaptor whose ubiquitin-interacting motif targets ubiquitylation by Nedd4. Traffic 3, 740-751.

Kim, W., et al., 2011. Systematic and quantitative assessment of the ubiquitin-modified proteome. Mol. Cell 44, 325-340.

Kim, K.S., et al., 2012. Hippocalcin and KCNQ channels contribute to the kinetics of the slow afterhyperpolarization. Biophys. J. 103, 2446-2454.

Kim, H.J., et al., 2016a. Protein arginine methylation facilitates KCNQ channel-PIP2 interaction leading to seizure suppression. elife 5 .

Kim, K.S., et al., 2016b. The voltage activation of cortical KCNQ channels depends on global PIP2 levels. Biophys. J. 110, 1089-1098.

Kitada, T., et al., 2007. Impaired dopamine release and synaptic plasticity in the striatum of PINK1-deficient mice. Proc. Natl. Acad. Sci. U. S. A. 104, 11441-11446.

Kobayashi, M., et al., 2005. Hippocalcin-deficient mice display a defect in cAMP response element-binding protein activation associated with impaired spatial and associative memory. Neuroscience $133,471-484$.

Kobayashi, M., et al., 2012. Hippocalcin mediates calcium-dependent translocation of brain-type creatine kinase (BB-CK) in hippocampal neurons. Biochem. Biophys. Res. Commun. 429, 142-147.

Kofoed, E.M., Vance, R.E., 2011. Innate immune recognition of bacterial ligands by NAIPs determines inflammasome specificity. Nature 477, 592-595.

Krause, M., et al., 2002. Functional specificity of G alpha $\mathrm{q}$ and $\mathrm{G}$ alpha 11 in the cholinergic and glutamatergic modulation of potassium currents and excitability in hippocampal neurons. J. Neurosci. 22, 666-673.

Krishnan, A., et al., 2009. Hippocalcin, new Ca(2+) sensor of a ROS-GC subfamily member, ONE-GC, membrane guanylate cyclase transduction system. Mol. Cell. Biochem. 325, 1-14.

Lafreniere, R.G., Rouleau, G.A., 2011. Migraine: Role of the TRESK two-pore potassium channel. Int. J. Biochem. Cell Biol. 43, 1533-1536.

Lai, Y.C., et al., 2015. Phosphoproteomic screening identifies Rab GTPases as novel downstream targets of PINK1. EMBO J. 34, 2840-2861.

Larsson, H.P., 2013. What determines the kinetics of the slow afterhyperpolarization (sAHP) in neurons? Biophys. J. 104, 281-283.

Lastres-Becker, I., et al., 2016. Mammalian ataxin-2 modulates translation control at the pre-initiation complex via PI3K/mTOR and is induced by starvation. Biochim. Biophys. Acta 1862, 1558-1569.

Leroy, E., et al., 1998. The ubiquitin pathway in Parkinson's disease. Nature 395, 451-452.

Li, S., et al., 2018. Regulation of smoothened ubiquitination and cell surface expression by a Cul4-DDB1-Gbeta E3 ubiquitin ligase complex. J. Cell Sci. 131 (pii: jcs218016).

Lin, M.T., Beal, M.F., 2006. Mitochondrial dysfunction and oxidative stress in neurodegenerative diseases. Nature 443, 787-795.

Liss, B., et al., 2005. K-ATP channels promote the differential degeneration of dopaminergic midbrain neurons. Nat. Neurosci. 8, 1742-1751.

Liu, Y., et al., 2007. Calcium influx through L-type channels generates protein kinase M to induce burst firing of dopamine cells in the rat ventral tegmental area. J. Biol. Chem. $282,8594-8603$

Liu, L., et al., 2012. Sirtuin 2 (SIRT2) enhances 1-methyl-4-phenyl-1,2,3,6-tetrahydropyridine (MPTP)-induced nigrostriatal damage via deacetylating forkhead box O3a (Foxo3a) and activating Bim protein. J. Biol. Chem. 287, 32307-32311.

Livak, K.J., Schmittgen, T.D., 2001. Analysis of relative gene expression data using realtime quantitative PCR and the 2(-Delta Delta C(T)) method. Methods 25, 402-408.

Lui, T.T., et al., 2011. The ubiquitin-specific protease USP34 regulates axin stability and Wnt/beta-catenin signaling. Mol. Cell. Biol. 31, 2053-2065.

Luthi-Carter, R., et al., 2000. Decreased expression of striatal signaling genes in a mouse model of Huntington's disease. Hum. Mol. Genet. 9, 1259-1271.

Luthi-Carter, R., et al., 2002. Dysregulation of gene expression in the R6/2 model of polyglutamine disease: parallel changes in muscle and brain. Hum. Mol. Genet. 11, $1911-1926$.

Macrez-Lepretre, N., et al., 1997. Distinct functions of Gq and G11 proteins in coupling alpha1-adrenoreceptors to $\mathrm{Ca} 2+$ release and $\mathrm{Ca} 2+$ entry in rat portal vein myocytes. J. Biol. Chem. 272, 5261-5268.

Madison, D.V., Nicoll, R.A., 1984. Control of the repetitive discharge of rat CA 1 pyramidal neurones in vitro. J. Physiol. 354, 319-331.

Mammen, A., et al., 2004. Hippocalcin in the olfactory epithelium: a mediator of second messenger signaling. Biochem. Biophys. Res. Commun. 322, 1131-1139.

Manzanillo, P.S., et al., 2013. The ubiquitin ligase parkin mediates resistance to intracellular pathogens. Nature 501, 512-516.

Martella, G., et al., 2009. Enhanced sensitivity to group II mGlu receptor activation at corticostriatal synapses in mice lacking the familial parkinsonism-linked genes PINK1 
or Parkin. Exp. Neurol. 215, 388-396.

Martinez, A., et al., 2017. Quantitative proteomic analysis of parkin substrates in Drosophila neurons. Mol. Neurodegener. 12, 29.

Martinez, A., et al., 2018. Neuronal proteomic analysis of the Ubiquitinated substrates of the disease-linked E3 ligases parkin and Ube3a. Biomed. Res. Int. 2018, 3180413.

Matschke, L.A., et al., 2015. A concerted action of L- and T-type $\mathrm{Ca}(2+)$ channels regulates locus coeruleus pacemaking. Mol. Cell. Neurosci. 68, 293-302.

Matschke, L.A., et al., 2018. Calcium-activated SK potassium channels are key modulators of the pacemaker frequency in locus coeruleus neurons. Mol. Cell. Neurosci., vol. 88, 330-341.

Mercer, E.A., et al., 2000. NAIP interacts with hippocalcin and protects neurons against calcium-induced cell death through caspase-3-dependent and -independent pathways. ЕMBO J. 19, 3597-3607.

Minowa-Nozawa, A., et al., 2017. Rab35 GTPase recruits NDP52 to autophagy targets. EMBO J. 36, 2790-2807.

Miura, M., et al., 2002. Group I metabotropic glutamate receptor signaling via Galpha q/ Galpha 11 secures the induction of long-term potentiation in the hippocampal area CA1. J. Neurosci. 22, 8379-8390.

Moseley, A.E., et al., 2003. The Na,K-ATPase alpha 2 isoform is expressed in neurons, and its absence disrupts neuronal activity in newborn mice. J. Biol. Chem. 278, 5317-5324.

Mouton-Liger, F., et al., 2018. Parkin deficiency modulates NLRP3 inflammasome activation by attenuating an A20-dependent negative feedback loop. Glia. https://doi. org/10.1002/glia.23337. [Epub ahead of print].

Myllykoski, M., et al., 2012. The N-terminal domain of the myelin enzyme 2',3'-cyclic nucleotide 3 '-phosphodiesterase: direct molecular interaction with the calcium sensor calmodulin. J. Neurochem. 123, 515-524.

Nesbit, M.A., et al., 2013. Mutations affecting G-protein subunit alpha11 in hypercalcemia and hypocalcemia. N. Engl. J. Med. 368, 2476-2486.

Okatsu, K., et al., 2012. Mitochondrial hexokinase HKI is a novel substrate of the Parkin ubiquitin ligase. Biochem. Biophys. Res. Commun. 428, 197-202.

Ordureau, A., et al., 2015a. Defining roles of PARKIN and ubiquitin phosphorylation by PINK1 in mitochondrial quality control using a ubiquitin replacement strategy. Proc Natl. Acad. Sci. U. S. A. 112, 6637-6642.

Ordureau, A., et al., 2015b. Quantifying ubiquitin signaling. Mol. Cell 58, 660-676.

Ordureau, A., et al., 2018. Dynamics of PARKIN-dependent mitochondrial ubiquitylation in induced neurons and model systems revealed by digital snapshot proteomics. Mol. Cell 70, 211-227 e8.

Outeiro, T.F., et al., 2007. Sirtuin 2 inhibitors rescue alpha-synuclein-mediated toxicity in models of Parkinson's disease. Science 317, 516-519.

Pahlavan, S., et al., 2012. Galphaq and Galpha11 contribute to the maintenance of cellular electrophysiology and $\mathrm{Ca} 2+$ handling in ventricular cardiomyocytes. Cardiovasc. Res. 95, 48-58.

Palacino, J.J., et al., 2004. Mitochondrial dysfunction and oxidative damage in parkindeficient mice. J. Biol. Chem. 279, 18614-18622.

Palmer, C.L., et al., 2005. Hippocalcin functions as a calcium sensor in hippocampal LTD. Neuron 47, 487-494.

Pareja, F., et al., 2012. Deubiquitination of EGFR by Cezanne-1 contributes to cancer progression. Oncogene 31, 4599-4608.

Pedarzani, P., Storm, J.F., 1993. PKA mediates the effects of monoamine transmitters on the $\mathrm{K}+$ current underlying the slow spike frequency adaptation in hippocampal neurons. Neuron 11, 1023-1035.

Periquet, M., et al., 2005. Proteomic analysis of parkin knockout mice: alterations in energy metabolism, protein handling and synaptic function. J. Neurochem. 95, $1259-1276$.

Pino, E., et al., 2014. FOXO3 determines the accumulation of alpha-synuclein and controls the fate of dopaminergic neurons in the substantia nigra. Hum. Mol. Genet. 23, $1435-1452$.

Poalas, K., et al., 2013. Negative regulation of NF-kappaB signaling in T lymphocytes by the ubiquitin-specific protease USP34. Cell Commun. Signal. 11, 25.

Ramaker, J.M., et al., 2013. Amyloid precursor proteins interact with the heterotrimeric G protein go in the control of neuronal migration. J. Neurosci. 33, 10165-10181.

Reetz, K., et al., 2008. Limbic and frontal cortical degeneration is associated with psy chiatric symptoms in PINK1 mutation carriers. Biol. Psychiatry 64, 241-247.

Reyes Ruiz, V.M., et al., 2017. Broad detection of bacterial type III secretion system and flagellin proteins by the human NAIP/NLRC4 inflammasome. Proc. Natl. Acad. Sci. U. S. A. $114,13242-13247$.

Rial, D., et al., 2014. Behavioral phenotyping of Parkin-deficient mice: looking for early preclinical features of Parkinson's disease. PLoS One, vol. 9, e114216.

Rojansky, R., et al., 2016. Elimination of paternal mitochondria in mouse embryos occurs through autophagic degradation dependent on PARKIN and MUL1. elife 5.

Roszko, K.L., et al., 2017. Knockin mouse with mutant Galpha11 mimics human inherited hypocalcemia and is rescued by pharmacologic inhibitors. JCI insight 2, e91079.

Rouzier, C., et al., 2017. A novel CISD2 mutation associated with a classical Wolfram syndrome phenotype alters $\mathrm{Ca} 2+$ homeostasis and ER-mitochondria interactions. Hum. Mol. Genet. 26, 1599-1611.

Saitoh, S., et al., 1993. Distribution of hippocalcin mRNA and immunoreactivity in rat brain. Neurosci. Lett. 157, 107-110.

Sallese, M., et al., 2000. Regulation of G protein-coupled receptor kinase subtypes by calcium sensor proteins. Biochim. Biophys. Acta 1498, 112-121.

Sarraf, S.A., et al., 2013. Landscape of the PARKIN-dependent ubiquitylome in response to mitochondrial depolarization. Nature 496, 372-376.

Savitt, J.M., et al., 2006. Diagnosis and treatment of Parkinson disease: molecules to medicine. J. Clin. Invest. 116, 1744-1754.

Schwindt, P.C., et al., 1988. Slow conductances in neurons from cat sensorimotor cortex in vitro and their role in slow excitability changes. J. Neurophysiol. 59, 450-467. Seirafi, M., et al., 2015. Parkin structure and function. FEBS J. 282, 2076-2088.

Shen, Z.Q., et al., 2017. CISD2 Haploinsufficiency disrupts calcium homeostasis, causes nonalcoholic fatty liver disease, and promotes hepatocellular carcinoma. Cell Rep. $21,2198-2211$.

Sliter, D.A., et al., 2018. Parkin and PINK1 mitigate STING-induced inflammation. Nature $561,258-262$

Soman, S., et al., 2017. Inhibition of the mitochondrial calcium uniporter rescues dopaminergic neurons in pink1(-/-) zebrafish. Eur. J. Neurosci. 45, 528-535.

Song, P., et al., 2016. Parkin modulates endosomal organization and function of the EndoLysosomal pathway. J. Neurosci. 36, 2425-2437.

Srivastava, A., et al., 2011. The relation between depression and parkin genotype: the CORE-PD study. Parkinsonism Relat. Disord. 17, 740-744.

von Stechow, L., et al., 2015. The E3 ubiquitin ligase ARIH1 protects against genotoxic stress by initiating a 4EHP-mediated mRNA translation arrest. Mol. Cell. Biol., vol. $35,1254-1268$.

Subramaniam, M., et al., 2014. Mutant alpha-synuclein enhances firing frequencies in dopamine substantia nigra neurons by oxidative impairment of A-type potassium channels. J. Neurosci. 34, 13586-13599.

Surmeier, D.J., et al., 2012. Physiological phenotype and vulnerability in Parkinson's disease. Cold Spring Harbor Perspect. Med. 2, a009290.

Sy, S.M., et al., 2013. The ubiquitin specific protease USP34 promotes ubiquitin signaling at DNA double-strand breaks. Nucleic Acids Res. 41, 8572-8580.

Szego, E.M., et al., 2017. Sirtuin 2 enhances dopaminergic differentiation via the AKT/ GSK-3beta/beta-catenin pathway. Neurobiol. Aging 56, 7-16.

Tashiro, K., et al., 2006. Suppression of the ligand-mediated down-regulation of epidermal growth factor receptor by Ymer, a novel tyrosine-phosphorylated and ubiquitinated protein. J. Biol. Chem. 281, 24612-24622.

Teckchandani, A., et al., 2014. Cullin 5 destabilizes Cas to inhibit Src-dependent cell transformation. J. Cell Sci. 127, 509-520.

Tiwari, M.N., et al., 2018. Differential contributions of $\mathrm{Ca}(2+)$-activated $\mathrm{K}(+)$ channels and $\mathrm{Na}(+) / \mathrm{K}(+)$-ATPases to the generation of the slow afterhyperpolarization in CA1 pyramidal cells. Hippocampus 28, 338-357.

Torres-Odio, S., et al., 2017. Progression of pathology in PINK1-deficient mouse brain from splicing via ubiquitination, ER stress, and mitophagy changes to neuroinflammation. J. Neuroinflammation 14,154

Tran, T.A., et al., 2011. Lipopolysaccharide and tumor necrosis factor regulate Parkin expression via nuclear factor-kappa B. PLoS One 6, e23660.

Tsukiyama, T., et al., 2012. Ymer acts as a multifunctional regulator in nuclear factorkappaB and Fas signaling pathways. Mol. Med. 18, 587-597.

Tzingounis, A.V., et al., 2007. Hippocalcin gates the calcium activation of the slow after hyperpolarization in hippocampal pyramidal cells. Neuron 53, 487-493.

Udeshi, N.D., et al., 2013. Large-scale identification of ubiquitination sites by mass spectrometry. Nat. Protoc. 8, 1950-1960.

Villalobos, C., Andrade, R., 2010. Visinin-like neuronal calcium sensor proteins regulate the slow calcium-activated after hyperpolarizing current in the rat cerebral cortex. J. Neurosci. 30, 14361-14365.

Vingill, S., et al., 2016. Loss of FBXO7 (PARK15) results in reduced proteasome activity and models a parkinsonism-like phenotype in mice. EMBO J. 35, 2008-2025.

Vives-Bauza, C., et al., 2010. PINK1-dependent recruitment of Parkin to mitochondria in mitophagy. In: Proceedings of the National Academy of Sciences of the United States of America. vol. 107. pp. 378-383.

Von Coelln, R., et al., 2004. Loss of locus coeruleus neurons and reduced startle in parkin null mice. Proc. Natl. Acad. Sci. U. S. A. 101, 10744-10749.

Wang, H., et al., 2011. Parkin ubiquitinates Drp1 for proteasome-dependent degradation: implication of dysregulated mitochondrial dynamics in Parkinson disease. J. Biol. Chem. 286, 11649-11658.

Wang, B., et al., 2017. TRAF2 and OTUD7B govern a ubiquitin-dependent switch tha regulates mTORC2 signalling. Nature 545, 365-369.

Xin, D., et al., 2018. Parkin negatively regulates the antiviral signaling pathway by targeting TRAF3 for degradation. J. Biol. Chem. 293, 11996-12010.

Yamano, K., et al., 2018. Endosomal Rab cycles regulate Parkin-mediated mitophagy. elife 7

Zhao, X.S., et al., 2001. Plasticity and adaptation of $\mathrm{Ca} 2+$ signaling and Ca2 + - dependent exocytosis in SERCA2(+/-) mice. EMBO J. 20, 2680-2689.

Zhao, Y., et al., 2011. The NLRC4 inflammasome receptors for bacterial flagellin and type III secretion apparatus. Nature 477, 596-600.

Zhong, Z., et al., 2016. NF-kappaB restricts inflammasome activation via elimination of damaged mitochondria. Cell 164, 896-910.

Zhu, Z., et al., 2016. Cutting edge: a cullin-5-TRAF6 interaction promotes TRAF6 polyubiquitination and lipopolysaccharide Signaling. J. Immunol. 197, 21-26.

Ziviani, E., et al., 2010. Drosophila parkin requires PINK1 for mitochondrial translocation and ubiquitinates mitofusin. Proc. Natl Acad. Sci. USA 107, 5018-5023. 


\subsection{Written declarations}

I herewith declare that I have not previously participated in any doctoral examination procedure in a mathematics or natural science discipline.

Frankfurt am Main,

Date

Signature

\section{$\underline{\text { Author's Declaration }}$}

I herewith declare that I have produced my doctoral dissertation on the topic of:

\section{"Mitochondrial dysfunction and its consequences in the context of neurodegeneration"}

independently and using only the tools indicated therein. In particular, all references borrowed from external sources are clearly acknowledged and identified.

I confirm that I have respected the principles of good scientific practice and have not made use of the services of any commercial agency in respect of my doctorate.

Frankfurt am Main,

Date 
10.5 Curriculum Vitae

\section{Jana Key}

Weingartenstraße 75

65795 Hattersheim

+4915775221845

key@stud.uni-frankfurt.de

\section{PERSONAL INFORMATION}

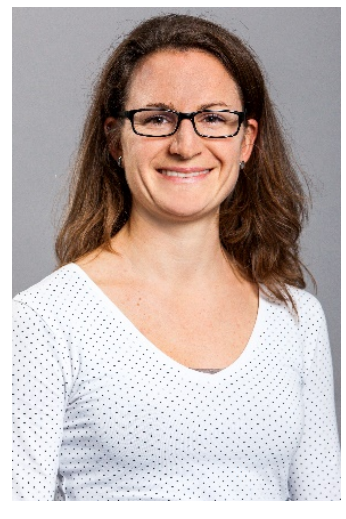

Birth:

Citizenship:

5. September 1988 in Bergisch-Gladbach

German

EDUCATION

2017-Present

$2015-2017$

$2012-2015$

$2009-2012$

$2007-2008$

$2004-2007$
UNIVERSITY HOSPITAL FRANKFURT AM MAIN

PhD Student in experimental neurology, Prof. Auburger laboratory

\section{GOETHE-UNIVERSITY FRANKFURT AM MAIN}

Master Program Interdisciplinary Neuroscience, Grade 1.3 (A)

Master thesis on mitochondrial dysfunctions in

neurodegenerative diseases considering innate immune

system activation

Supervisor: Prof. Dr. Georg Auburger

PHILIPPS UNIVERSITY MARBURG

Bachelor Human Biology/Biomedical Science, Grade 1.5 (A)

Specialisation on Neuroscience from $3^{\text {rd }}$ Semester on

Bachelor thesis on neuroinflammation in Alzheimer's disease Title: Establishment of a MIF- deleted human neuroblastoma cell line by usage of the CRISPR/Cas9-System

Supervisor: Prof. Dr. Michael Bacher

BFS FOR KRANKENPFLEGE OF THE BAVARIAN RED CROSS

Apprenticeship as a nurse

AU PAIR STAY IN LONDON

ALEXANDER VON HUMBOLDT GYMNASIUM SCHWEINFURT

Allgemeine Hochschulreife (Abitur), Grade 2.0 (B)

Major subjects: Biology, English, Chemistry 


\section{PROFESSIONAL EXPERIENCES}

October 2018

October 2016

June 2016
Targeting Mitochondria Conference Berlin, Talk on 'Neuroinflammation triggered by mitochondrial dysfunctions'

Symposium: 'Beyond amyloid', Hannover, Germany

Biennial meeting Rhine Main Neuroscience Network, Oberwesel, Germany

\section{PUBLICATIONS}

2021

2021

2020

2020

2019

2019
Journal of Immunology, ji2001016

Loss of mitochondrial protease CLPP activates type I interferon responses through the mtDNA-cGAS-STING signaling axis S. Torres-Odio, Y. Lei, S. Gispert, A. Maletzko, J. Key, S. Menissy, I. Wittig, G. Auburger, A.P. West

\section{Neurobiology of Disease, 105289}

Atxn2-CAG100-KnockIn mouse spinal cord shows progressive TDP43 pathology associated with cholesterol biosynthesis suppression

J. Canet-Pons, N.E. Sen, A. Arsovic, L.E. Almaguer-Mederos, M. Halbach, J. Key, C. Döring, A. Kerksiek, G. Picchiarelli, R. Cassel, F. René, S. Dieterlé, N. Fuchs, R. König, L. Dupuis, D. Lütjohann, S. Gispert, G. Auburger

International Journal of Molecular Science, 21(14), 5124

Mid-Gestation lethality ofAtxn2l-Ablated Mice

J. Key, P. N. Harter, N-E. Sen, E. Gradhand, G. Auburger, S. Gispert

Neurogenetics, 21:187-203

Loss of mitochondrial ClpP, Lonp1 and Tfam triggers transcriptional induction of Rnf213, a susceptibility factor for Moyamoya disease

J. Key, A. Maletzko, A. Kohli, S. Gispert, S. Torres-Odio, I. Wittig, J. Heidler, C. Bárcena, C. López-Otín, Y. Lei, A.P. West, G. Auburger

International Journal of Molecular Science, 20(18)

Global Proteome of LonP1+/- Mouse Embryonal Fibroblasts Reveals Impact on Respiratory Chain, but No Interdependence between Eral1 and Mitoribosomes.

J. Key, A. Kohli, C. Bárcena, C. López-Otín, J. Heidler, I. Wittig, G. Auburger.

International Journal of Molecular Science, 20(13)

SerThr-PhosphoProteome of Brain from Aged PINK1KO+A53T-SNCA Mice Reveals pT1928-MAP1B and pS3781ANK2 Deficits, as Hub between Autophagy and Synapse Changes.

G. Auburger, S. Gispert, S. Torres-Odio, M. Jendrach, N. Brehm, J. Canet-Pons, J. Key, NE Sen 
Neurobiology of disease, 127:114-130

Ubiquitylome profiling of Parkin-null brain reveals dysregulation of calcium homeostasis factors ATP1A2, Hippocalcin and GNA11, reflected by altered firing of noradrenergic neurons. J. Key; AK Mueller; S. Gispert; L. Matschke; I. Wittig; O. Corti; C. Muench, N. Decher; G. Auburger

Journal of Neuroinflammation, 14(1):154

Progression of pathology in PINK1-deficient mouse brain from splicing via ubiquitination and mitophagy changes to neuroinflammation

S. Torres-Odio, J. Key, H.H. Hoepken, J. Canet-Pons, M. Walter, B. Morales-Gordo, S. Gispert, G. Auburger

Movement Disorders; 32 (suppl 2); 2017

CLPP-Knock-Out mouse brain shows accumulation of mitoribosomes

J. Key, J. Heidler, S. Torres-Odio, G. Auburger, I. Wittig, S. Gispert

\section{QUALIFICATIONS}

Languages

Computing

Other

REFERENCES
German: native

English: fluent

Microsoft Word, Excel, Powerpoint, basic in Matlab, Photoshop, Graphpad Prism, STRING

Felasa course $(A, D)$
Bachelor Project:

Master Project:

PhD Project:
Prof. Dr. Michael Bacher, Department of Immunology, Philipps University Marburg

Prof. Dr. Georg Auburger, Department of experimental neurology, Goethe-University Frankfurt

Prof. Dr. Enrico Schleiff, Department of Biosciences, GoetheUniversity Frankfurt

Prof. Dr. Georg Auburger, Department of experimental neurology, Goethe-University Frankfurt

Frankfurt / Main, 27 March 2021 